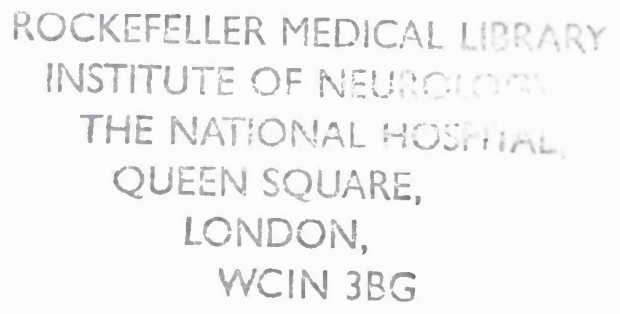

\title{
THE ROLE OF THE PEDUNCULOPONTINE REGION IN BASAL GANGLIA MECHANISMS OF AKINESIA
}

LISA EDANA MUNRO-DAVIES.

MB.BS. BSc. F.R.C.S.

A thesis submitted to the University of London for the degree of Doctor

of

Medicine.

March 1999. 
ProQuest Number: U145711

All rights reserved

INFORMATION TO ALL USERS

The quality of this reproduction is dependent upon the quality of the copy submitted.

In the unlikely event that the author did not send a complete manuscript and there are missing pages, these will be noted. Also, if material had to be removed, a note will indicate the deletion.

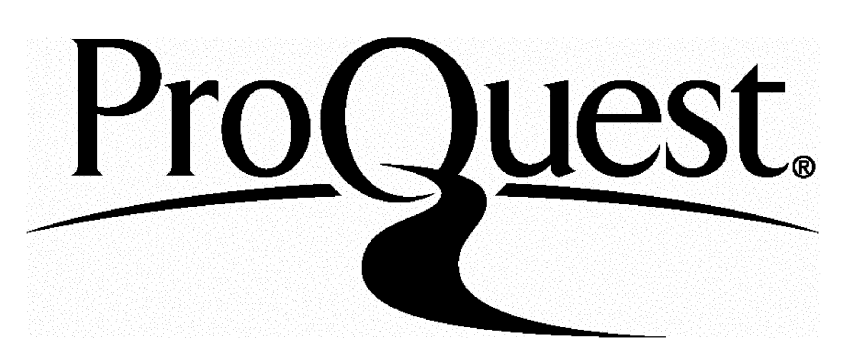

ProQuest U145711

Published by ProQuest LLC(2016). Copyright of the Dissertation is held by the Author.

All rights reserved.

This work is protected against unauthorized copying under Title 17, United States Code. Microform Edition (c) ProQuest LLC.

\author{
ProQuest LLC \\ 789 East Eisenhower Parkway \\ P.O. Box 1346 \\ Ann Arbor, MI 48106-1346
}




\section{TABLE OF CONTENTS}

Acknowledgements $\quad 5$

Declaration 6

Abbreviations $\quad 7$

Abstract $\quad 8$

\section{CHAPTER ONE - Introduction}

$\begin{array}{ll}\text { Introduction } & 11\end{array}$

Aetiology of Parkinson's Disease 11

Pathology of Parkinson's Disease 15

Motor Circuits of the Basal Ganglia 18

Clinical Features of Parkinson's Disease 23

Medical Therapy of Parkinson's Disease 25

Surgical Treatment of Parkinson's Disease 27

Anatomy of the Pedunculopontine Nucleus 44

The Role of the Pedunculopontine Nucleus in Locomotion 49

The Pedunculopontine Nucleus and Parkinson's Disease 52

CHAPTER TWO - Thermocoagulative lesions of the PPN in the normal sub-human primate.

Introduction $\quad 55$

Materials and Methods $\quad 56$

Results $\quad 69$

CHAPTER THREE - Designing a stereotactic frame for use in the sub-

human primate.

The Need for a New Frame $\quad 85$

Introduction to Stereotaxis $\quad 86$

Animal Stereotaxis $\quad 87$

Materials and Design $\quad 88$

Target Acquisition $\quad 94$

CHAPTER FOUR - Excitotoxic lesions of the PPN in the normal subhuman primate.

Introduction $\quad 99$

Materials and Methods $\quad 100$

Results 113

CHAPTER FIVE - Discussion

Discussion 132

$\begin{array}{ll}\text { Conclusions } & 140\end{array}$

References 142

APPENDIX 1 - Calculation of PPN Co-ordinates in Monkeys 1 - 6175

APPENDIX 2 - Calculation of PPN Co-ordinates in Monkeys A - E 185

APPENDIX 3 - Authors publications cited in text 196 


\section{ILLUSTRATIONS}

Fig 1.1aNormal Basal Ganglia Connections 20

Fig 1.1bBasal Ganglia Connections in Parkinson's Disease $\quad 21$

Fig 1.2 Early Surgical Targets in Parkinson's Disease $\quad 32$

Fig 1.3 Stereoencephalotome of Spiegel and Wycis 36

Fig 1.4 Leksell's Posteroventral Pallidotomy Target 41

Fig 2.1aOperative Ventriculogram in Thermocoagulative Lesioning 63

Fig 2.1bTracing of Ventriculogram 63

Fig 2.2 Lateral Radiograph with Electrode at Target 64

Fig 2.3a-f Histology in Monkeys 1to $6 \quad 66-68$

Fig 2.4 Individual Activity Counts Post Unilateral Thermocoagulative Lesions of the PPN $\quad 80$

Fig 2.5 Averaged Activity Counts Post Unilateral Thermocoagulative Lesions of the PPN

Fig 2.6 Individual Activity Counts Post Bilateral Thermocoagulative Lesions of the PPN

Fig 2.7 Averaged Activity Counts Post Bilateral Thermocoagulative Lesions of the PPN

Fig 3.1aSuperior View of Frame $\quad 91$

Fig 3.1bLateral View of Frame $\quad 91$

Fig 3.1cPosterior View of Frame $\quad 92$

Fig 3.2 Fixation Mechanisms of Frame 92

Fig 3.3 Linear Scale Sidearm of frame in situ 93

Fig 3.4aHamilton Syringe Zeroed 95

Fig 3.4bClose Up of Zeroed Hamilton Syringe 96

Fig 3.5 Targeting Positions Demonstrated 96

Fig 4.1aVentriculogram of Monkey in Stereotactic Frame 108

Fig 4.1bTracing of Ventriculogram 108

Fig 4.2 Lateral Radiograph with Hamilton Syringe at Target 109

Fig 4.3a-eHistological sections from Monkeys A-E 110-112

Fig 4.4 Individual Activity Counts Following Unilateral Cytotoxic Lesions of the PPN 129

Fig 4.5 Individual Activity Counts Following Bilateral Cytotoxic

Lesions of the PPN 


\section{TABLES}

Table 2.1 Data Correction and Target Calculation in Thermocoagulative Lesioning

Table 2.2 Individual and Averaged Activity Counts Following Unilateral Thermocoagulative PPN Lesions

Table 2.3 Individual and Averaged Activity Counts Following Bilateral Thermocoagulative PPN Lesions

Table 3.1 Data Correction and Target Calculation in Cytotoxic Lesioning 97

Table 4.1 Clarkes Rating Scale for Parkinsonism in Monkeys

Table 4.2 Individual Activity Counts Following Unilateral Cytotoxic PPN Lesion

Table 4.3 Individual Activity Counts Following Staged Bilateral Cytotoxic PPN Lesions

Table 4.4 Individual Activity Counts Following Simultaneous Bilateral Cytotoxic PPN Lesions

Table 4.5 Clarke's Scoring Pre and Post PPN Lesioning 


\section{ACKNOWLEDGEMENTS}

I thank Professor J F Stein of the University Laboratory of Physiology, University of Oxford for the provision of the research facilities to pursue this project. I also thank the Medical Research Council and the Norman Collisson Foundation for their financial support.

I am particularly grateful to $\mathrm{Mr} \mathrm{T} \mathrm{Z}$ Aziz and Prof $\mathrm{J}$ F Stein for their meticulous supervision, advice and unstinting constructive criticisms throughout the execution and recording of this work.

I also thank Mr Jonathan Winter and Mrs Jayne Watson for their invaluable technical assistance.

I am extremely grateful to Dr Daniel Woolpert for his efforts as my cosupervisor in this project.

I also thank all those in the Animal Unit and the Department of Photography of the University Laboratory of Physiology for their help.

This work is dedicated to my mother, Margaret, and my husband Peter for his unfaltering support. 


\section{DECLARATION}

No portion of the work referred to in this thesis has been submitted in support of an application for another degree or qualification of this or any other university or other institution of learning. 


\section{ABBREVIATIONS}

\section{Anatomical :}

$\mathrm{AC}$ - anterior commisure.

AC-PC - intercommisural distance / line.

$\mathrm{BC}$ - brachium conjunctivum.

CAUD - caudate nucleus.

GP - globus pallidus.

GPI - lateral / external globus pallidus.

GPm - medial / internal globus pallidus.

IC - internal capsule.

MLR - mesencephalic locomotor region.

$\mathrm{NR}$ - red nucleus.

$\mathrm{PC}$ - posterior commisure.

PPNc - pars compacta of the pedunculopontine nucleus.

PPNd - pars dissipata of the pedunculopontine nucleus.

PPN - pedunculopontine nucleus.

PUT - putamen.

SMA - supplementary motor area.

SN - substantia nigra.

SNc - pars compacta of the substantia nigra.

$\mathrm{SNr}$ - pars reticulata of the substantia nigra.

STN - subthalamic nucleus.

VIM - ventro-intermedius nucleus of the thalamus.

VL - venterolateral nucleus of the thalamus.

VOP - ventro-oralis posterior nucleus of the thalamus.

\section{Non-Anatomical :}

Ach - acetylcholine.

$\mathrm{CO}$ - carbon monoxide.

COMT - catecholamethyltransferases.

CSF - cerebrospinal fluid.

CV - Cresyl Violet

2DG - 2-deoxy glucose.

ENK - enkephalin.

GABA - gamma amino butyric acid.

GLU - glutamate.

L-dopa - levodopa.

LFB - Luxol Fast Blue.

MAOI - monoamine oxidase inhibitor.

MER - microelectrode recording.

MPTP - 1-methyl-4-phenyl-1,2,3,6-tetrahydropyridine.

PD - Parkinson's disease.

PET - positron emission tomography.

PHNO - 4-propyl-9-hydroxynaphtoxazine.

PSP - progressive supranuclear palsy

PVP - posteroventral pallidotomy.

SP - substance $P$ 


\section{ABSTRACT}

There has in recent years been a resurgence of interest in the treatment of Parkinson's disease by stereotactic surgical techniques. The phenomenal success of levo-dopa in the 1960s led to the virtual disappearance of surgery for Parkinson's Disease. However, two decades after its introduction the problems associated with the long-term administration of levo-dopa became well recognized.

Over the same period the advance in our knowledge of the neural mechanisms underlying parkinsonian symptoms has been remarkable. Studies established that the loss of the nigro-striatal dopaminergic projection results in overactivity of the GPm and SNr inhibitory output, which in turn depresses the motor activity of thalamic and brainstem structures to which they have been shown to project, thus leading to the clinical manifestations of parkinsonism. It has long been assumed that the increased inhibitory output of the GPm acts via the thalamocortical feedback route to produce akinesia. However, this view fails to explain the clinical and experimental observation that thalamotomy, despite relieving tremor, rarely improves akinesia. Conversely medial pallidotomy may alleviate akinesia but has a lesser effect upon tremor, whereas high frequency stimulation or lesioning of the STN improves both symptoms. As thalamic lesioning does not affect the descending outputs of the basal ganglia, whereas pallidotomy and subthalamic nucleotomy do, a logical conclusion would be that overactivity of descending projections to the pedunculopontine area in the upper brainstem, rather than the overinhibition of the thalamic motor nuclei, is responsible for the akinesia of Parkinson's Disease. 
Therefore I have studied the effects of lesions of the PPN on movements in monkeys. The results establish that in the normal monkey a unilateral lesion of the PPN will result in a temporary akinetic state, whereas bilateral PPN lesions will generate a lasting Parkinsonian like akinesia. Results are consistent whether the lesioning method is by radiofrequency thermocoagulation, or by pressure injection of a neuron specific excitotoxic agent.

Clinically, I have worked with the Oxford Movement disorder group studying the effects of lesioning, and deep brain stimulation in the basal ganglia of Parkinsonian patients. 
CHAPTER ONE

INTRODUCTION 


\section{INTRODUCTION}

Parkinson's disease $(\mathrm{PD})$ is a progressive neurodegenerative disorder, which represents the most common movement disorder seen in neurological practice. Estimates of its prevalence vary between $84-164$ per 100,000 of a population, with an incidence of between 8.7-20 per 100,000 (Brewis 1966, Dupont 1977, Kurland 1958, Mutch et al. 1986). Racial and geographic factors may play a part in the variability of incidence and prevalence of PD as, rates are very low in Nigeria (Schoenberg et al. 1988) and China (Li et al. 1985), as compared to the UK (Mutch et al. 1986). Also, in an American survey the rate in AfroCaribbeans has been shown to be lower than in Caucasians (Kessler 1972).

\section{AETIOLOGY OF PARKINSON'S DISEASE}

To date the aetiology and pathogenesis of PD remains unknown. This is so despite great efforts expended over many years to find a cause in the hope of preventing it, or altering its progression. There is a consensus emerging that there are likely to be multiple aetiologies that may result in the clinical and pathological abnormalities common to the majority of patients with idiopathic PD. Aetiological studies so far performed fall into three distinct groups; genetic, age related and environmental investigations.

Genetic : Three lines of evidence have been proposed in favour of a genetic pre-disposition to PD. First, analysis of 100 consecutive cases of PD revealed that $24 \%$ had a positive family history (Bonifati et al 1995). Second, the high concordance rates found in twin studies (Marsden 1987, Ward et al 1983), may reflect a clinical underestimation of the incidence of PD, as recent studies using positron emission tomography (PET) of clinically unaffected cotwins suggest that a number may have presymptomatic PD (Burn et al 1992, Piccini et al 1997). Third, an increasing number of families are being reported who show apparent autosomal dominant inheritance of PD (Golbe et al 1996, Wszolek et al 1995). The investigation of one such family, the Contursi 
kindred, showing autosomal dominant inheritance of parkinsonism with Lewy body pathology, ultimately led to the mapping of a locus to chromosome 4q21-23, designated PD-1 (Polymeropoulos et al 1996). Subsequently, an alanine-to-threonine substitution in the $\alpha$-synuclein gene has been identified (Polymeropoulos et al 1997), and has been proposed as the causative mutation necessary for the development of PD. However, other studies dispute this theory and report no correlation between PD incidence and the $\alpha$-synuclein mutation (Vaughn et al 1998). The fuller and further assessment of this hypothesis forms the current main thrust of investigation into a possible genetic cause for PD.

Age : There is no doubt that with advancing age we see a progressive loss of dopaminergic cells in the substantia nigra, coupled to a loss in striatal dopamine, dopamine receptors and the synthesizing enzymes. These losses, however, are not profound and alone would certainly be insufficient to account for the $80 \%$ loss of dopamine production necessary for parkinsonism to become clinically manifest (Agid et al. 1987). Also, it has been demonstrated that the incidence of PD peaks at 60 years of age, and after 75 years falls dramatically (Koller W et al. 1986). If indeed there were an age related aetiology for PD there should be a linear increase in incidence with increasing age. Furthermore, the lack of concordance in twin studies with age also mitigates against this hypothesis as twins can be expected to age at similar rates. It may well be that age is an additional risk factor in the aetiology of PD rather than a causative one.

Environment : Current research has underlined the possibility of environmental toxins playing a significant role in the aetiology of Parkinson's disease. An infective cause for Parkinson's disease as distinct from postencephalitic parkinsonism has not been established. The work of Rajput et al (Koller et al. 1986, Rajput 1984, Rajput et al. 1986) would suggest that young onset Parkinson's disease is more common in rural environments in which the 
water supply was exclusively from wells. They postulate that drinking water may be a source of a common toxin, possibly pesticides. In this context the similarity of MPTP to paraquat is striking. Against a pesticide hypothesis is the follow up study (Zilker et al 1988) of survivors of paraquat ingestion. None of them subsequently developed parkinsonism. In another study into the high frequency of the parkinsonism/dementia complex in Guam (Spencer et al 1987) the condition was related to the ingestion of cycad, which contains beta$\mathrm{N}$-methyl-l-alanine, which causes basal ganglia dysfunction when fed to primates.

The interesting finding that cigarette smoking protects against later development of Parkinson's disease (Baron 1986) is in keeping with a neurotoxin hypothesis if one believes that it competes with a PD inducing toxin. Indeed, Yong and Perry (Yong \& Perry 1986) have shown that hydrazine which is present in tobacco smoke protects against MPTP toxicity in mice.

The work of Barbeau (Barbeau et al 1986) suggests that it is a combination of genetic and environmental factors that lead to an expression of the disease. In Quebec the incidence of PD is highest in rural areas with a high use of pesticides. Patients with the condition were also noted to have reduced levels of the hepatic detoxifying cytochrome P450 monoxygenases. It is perhaps this combination of genetic predisposition and the environment that leads to expression of the disease.

Specific neurotoxins that have previously been shown to produce akinetic syndromes include carbon monoxide (CO) and manganese. $\mathrm{CO}$ is a common environmental pollutant but it may also play an important role in cell signaling. Acute $\mathrm{CO}$ poisoning results in complexing of $\mathrm{CO}$ with the ferrous iron, protoporphyrin IX, and this prevents the carriage of oxygen. $\mathrm{CO}$ is also a potent inhibitor of cytochrome oxidase. Survivors of $\mathrm{CO}$ poisoning have been noted to develop parkinsonism including rigidity and tremor within a few days 
or weeks after exposure (Gordon 1965, Grinker 1926, Klawans et al 1982). Affected patients have shown necrosis of the GP on CT scanning. The first description of manganese inducing an akinetic rigid syndrome was when Couper (Couper 1837) described a patient who developed mild stiffness and rigidity while working in a French manganese mill. Since that time there have been many reports of manganism developing among individuals exposed to manganese dioxide ore. Exposure usually involves the inhalation of manganese dust and the duration of exposure may be between 6 months and 16 years. Manganese predominantly appears to affect the striatum and GP with sparing of the SN.

In 1917 there was an outbreak of an influenza-like illness in Austria and France, mortality was $30-40 \%$, and Parkinsonism developed in the majority of survivors over the next 10 years (Von Economo 1931, Ziegler 1928). The specific agent causing the so-called encephalitis lethargica epidemic was never identified, and by 1919 it had spread throughout Europe and reached North and Central America. Although there has been no outbreak of encephalitis lethargica since the 1920's, infection as a cause for PD has still attracted some attention. There are numerous anecdotal reports of infections, particularly encephalitis, being associated with parkinsonism. These include a wide variety of viruses, bacteria - including Borrelia burgdorferi (Lyme disease)- and even fungi, such as Cryptococcus or Aspergillus. However, there is no evidence to suggest that any of these are relevant to the vast majority of patients with idiopathic PD.

A recent study (DePalma et al 1998) examining the interactions between genetic and environmental factors in PD lists the following risk factors and odds ratio for each; rural living (3.62 risk), drinking well water (2.09 risk), exposure to pesticides (2.92 risk), exposure to copper sulphate (2.69 risk), and homozygous deletion of glutatione S-Transferase gene loci (1.8 risk). 
Negative associations that suggest protective roles, were found for smoking (0.54:1) and urban living (0.52:1).

Available data concerning the aetiology of PD implies that multiple aetiologies are more likely than a single common factor. It may be that the ability to identify subsets of patients with PD will accelerate research into aetiology and circumvent the problems of specific factors being obscured by group-to-group comparison.

\section{PATHOLOGY OF PARKINSON'S DISEASE}

The identification of the pathological changes associated with Parkinson's disease has gradually evolved over the 180 years subsequent to James Parkinson's initial description of the clinical condition in 1817. In 1893, Blocq and Marinescu described a case of hemiparkinsonism with a tuberculoma of the cerebral peduncle contralateral to the affected side. This led Brissaud to postulate the possible relevance of the substantia nigra to the disease.

In 1913 Lewy described the eosinophilic bodies that bear his name in the substantia innominata, and in 1919 Tretiakoff described the characteristic lesions of the substantia nigra. In 1921 Foix published a study of seven cases of Parkinson's disease and one postencephalitic parkinsonian patient. The parkinsonian brains showed loss of pigmented cells in the substantia nigra and in the latter, gross destruction of the nigra with inflammatory changes (vintage references cited by T Z Aziz 1992).

Hassler in 1938 (Hassler 1938) published a study of nine cases of Parkinson's disease. Lewy bodies were present in all, and the nigral lesions were virtually confined to the middle third of the pars compacta. Further studies have repeatedly confirmed these findings in association with variable striatal and pallidal lesions. 
It is now generally accepted that Lewy bodies are virtually pathognomonic of the disease and occur in the nigra, locus coeruleus, dorsal motor nucleus of the vagi, brain stem nuclei and in the lateral grey horn of the thoracic cord. Their presence is not, however, confined to cases of Parkinson's disease, occurring in 5\% of controls in the study by Lipkin (Lipkin 1959) and 4\% in that of Forno (Forno 1966). Thus, in a recent survey of contemporary neurologists, $76 \%$ agreed that the presence of Lewy bodies defined Parkinson's disease (Duvoisin 1989).

Histochemically, Lewy bodies contain sphingomyelin, free fatty acids and polysaccharides. Electron microscopic studies (Duffy \& Tennyson 1965) have shown the bodies to consist of filaments loosely packed in the outer zone and densely packed with granular material in the central core.

An understanding of the neurochemical changes that attended the pathological changes began to evolve in the 1960 's. In the normal rat brain high concentrations of dopamine were found in the striatum. When rats were reserpinised, dopamine levels fell and the rats became catatonic, a state that could be reversed with L-dopa. This led to initial trials of racemic dopa in Parkinson's disease. At the same time it was also demonstrated that striatal dopamine was decreased in parkinsonian brains (Ehringer \& Hornykiewicz 1960), and subsequent to this finding the understanding of the neurochemistry of Parkinson's disease has rapidly expanded. A brief summary is presented. Degeneration of the neurons of the substantia nigra pars compacta leads to loss of the nigrostriatal pathway causing striatal dopamine depletion. This is the change that is believed to underlie the motor symptomatology of parkinsonism. The striatal dopamine depletion is not uniform and is more marked in the putamen than the caudate (Nyberg et al. 1983). It follows that dopamine metabolites e.g. homovanillic acid and dihydroxyphenylacetic acid are also decreased in the striatum. The enzyme tyrosine hydroxylase which occurs in all dopaminergic neurons is also decreased. For parkinsonism to 
become manifest it has been estimated that $80 \%$ of nigral dopaminergic neurons must be lost (Agid et al. 1987).

Striatal dopamine receptors have been classified into D-1 receptors, which activate adenylate cyclase, and D-2 receptors which do not (Kebabian \& Calne 1979). D-2 receptors are believed to be the more important functionally in Parkinson's disease as D-2 specific agonists and antagonists affect the motor symptoms most profoundly. However, D-1 receptors may have a role in the behavioral aspects of the condition (Beaulieu 1987).

Other dopaminergic systems are affected in Parkinson's disease e.g. the mesolimbic system, the mesocorticolimbic system and brain stem and hypothalamic pathways. Also, the ventral tegmental area with ascending dopaminergic pathways to the nucleus accumbens, cortex and hippocampus, shows loss of cells, dopamine and tyrosine hydroxylase in PD. Hypothalamic dopamine also decreases. However, the condition is not associated with any particular endocrine aberration. In the brain stem there is noted to be cell loss in the locus coeruleus with subsequent dopamine loss.

A further finding in PD is that in the striatum there is an increase in cholinergic activity as indicated by increased choline acetyl-transferase activity.

The changes in GABA concentration in the striatum are the inverse of those seen for dopamine i.e. increased especially caudally. It is normal in other brain regions.

Furthermore, in parkinsonian patients there are significant changes in noradrenaline concentrations, falling to $40-70 \%$ below controls in the neocortex and limbic areas. Decreased levels are also demonstrable in the substantia nigra and cerebellar cortex and locus coeruleus. It is probable that noradrenergic pathways may facilitate dopaminergic motor pathways as some symptom alleviation has been reported with a specific noradrenergic precursor dihydroxyphenylserine (Narabayashi et al 1991). 
Changes in serotoninergic pathways have been reported; but they are not consistent and as yet have no clinical correlates.

\section{MOTOR CIRCUITS OF THE BASAL GANGLIA}

During the last two decades the establishment of powerful tracing techniques and cellular recording has led to a great increase in our knowledge of basal ganglia anatomy and physiology. The establishment of what is 'normal' has been complemented by increased knowledge regarding the abnormal functioning within these circuits in the case of PD, largely due to the development of a very good animal model of the disease in the form of the MPTP treated monkey. Thus today we have established schemes of the basal ganglia motor circuit in both health and PD. These will be outlined and their relevance to a possible role for the PPN elaborated on later.

The motor loop which passes through the basal ganglia includes the precentral motor areas (areas $4 \& 6$, supplementary motor area) and postcentral sensory fields $(3 a-b, 2,1)$, which project to the putamen in a somatotopically organized fashion. Detailed anatochemical studies have shown that putaminal output reaches the medial globus pallidus (GPm) and the substantia nigra reticulata (SNr) through two different projections which arise from separate putaminal neuronal populations - the Direct and the Indirect pathways. The direct monosynaptic pathway passes from putamen to GPm. It is GABAergic and colocalised with substance $\mathrm{P}$ and dynorphin and is excited by dopamine acting via $\mathrm{D} 1$ receptors. The indirect pathway arises from GABA/enkephalergic putaminal neurons and projects to the lateral globus pallidus (GPI). It is inhibited by dopamine acting via D2 receptors. The GP1 sends a GABAergic inhibitory projection to the sensorimotor region of the STN and also to GPm (Parent \& Hazrati 1993). The STN exerts a powerful excitatory drive on the GPm and SNr, which in turn project via inhibitory GABAergic efferents to the venterolateral thalamus, and thus on to the premotor cortices (Fig. 1.1a). The 
GPm also projects to the non-cholinergic portion of the PPN and the $\mathrm{SNr}$ sends efferents to the superior colliculus and also to the pedunculopontine region of the mesopontine tegmentum (Hikosaka \& Wurtz 1983, Scarnati 1996). In addition cholinergic, GABAergic and glutamatergic fibers project from the PPN and mesopontine tegmentum back to the STN (Bevan \& Bolam 1995), providing an anatomical basis for a possible functional interface between brainstem regions related to locomotion and posture and the basal ganglia.

The basic mechanisms underlying the cardinal features of parkinsonian syndromes have been elucidated using the MPTP monkey model of PD, and can be integrated in to the scheme of basal ganglia motor circuits outlined above. Loss of dopaminergic neurons in the SNc reduces the normal inhibition by the nigrostriatal dopaminergic pathway of the D2 receptors on GABAenkephalin neurons of the indirect pathway. Hence these increase their activity, thus overinhibiting the GPl. The inhibitory tone of the GPl on the STN is thus reduced and the STN increases its' activity thus overexciting the GPm and SNr which in turn over inhibit their targets in the thalamus and upper brainstem. Concurrently, the direct pathway is not stimulated by its' D1 receptors. Hyperactivity of the STN leads to excessive stimulation of its' efferent targets and this pathophysiology of the parkinsonian state has been shown by 2-deoxyglucose uptake and GADmRNA in situ hybridization studies in MPTP treated monkeys (Crossman et al 1985, Herrero et al 1996, Vila et al 1996)(Fig.1.1b). The role of increased STN activity in the pathogenesis of parkinsonian symptomatology is further underlined by the improvement in symptoms in MPTP treated monkeys following STN lesioning (Aziz et al 1991, Bergmann et al 1990, Guridi et al 1996). In addition to the improvement in symptomatology, it has been shown that STN lesions return the GABA activity in both the GPm and SNr towards normal levels in GAD mRNA studies (Guridi et al 1996). 
Figure 1.1a. A Simplified Diagram of Normal Basal Ganglia Function in the Control of Movement.

(GLU = glutamatergic / excitatory, GABA = GABAergic $/$ Inhibitory $\mathrm{DA}=$ dopaminergic $/$ Inhibitory)

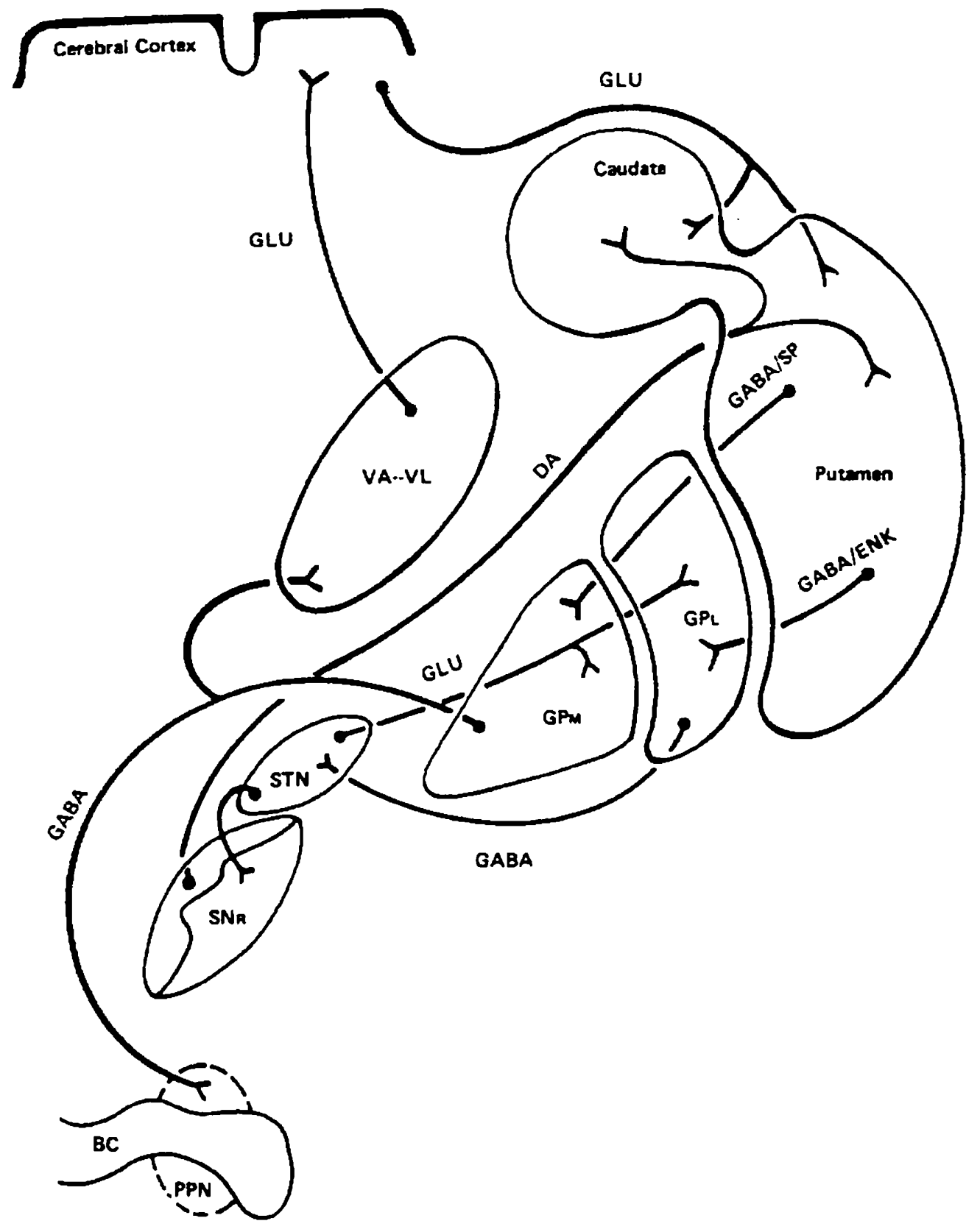


Figure 1.1b. In the Parkinsonian State, Decreased Activity in the DA Inhibitory Projection Leads to an Increase in the Putaminolateral Projection. STN Then Exerts Excessive Excitatory Drive to GPm with Excessive Thalamic and PPN Inhibition.

( Broken Line $=$ Underactive, Heavy Line $=$ Overactive.)

(Taken from Aziz et al 1992)

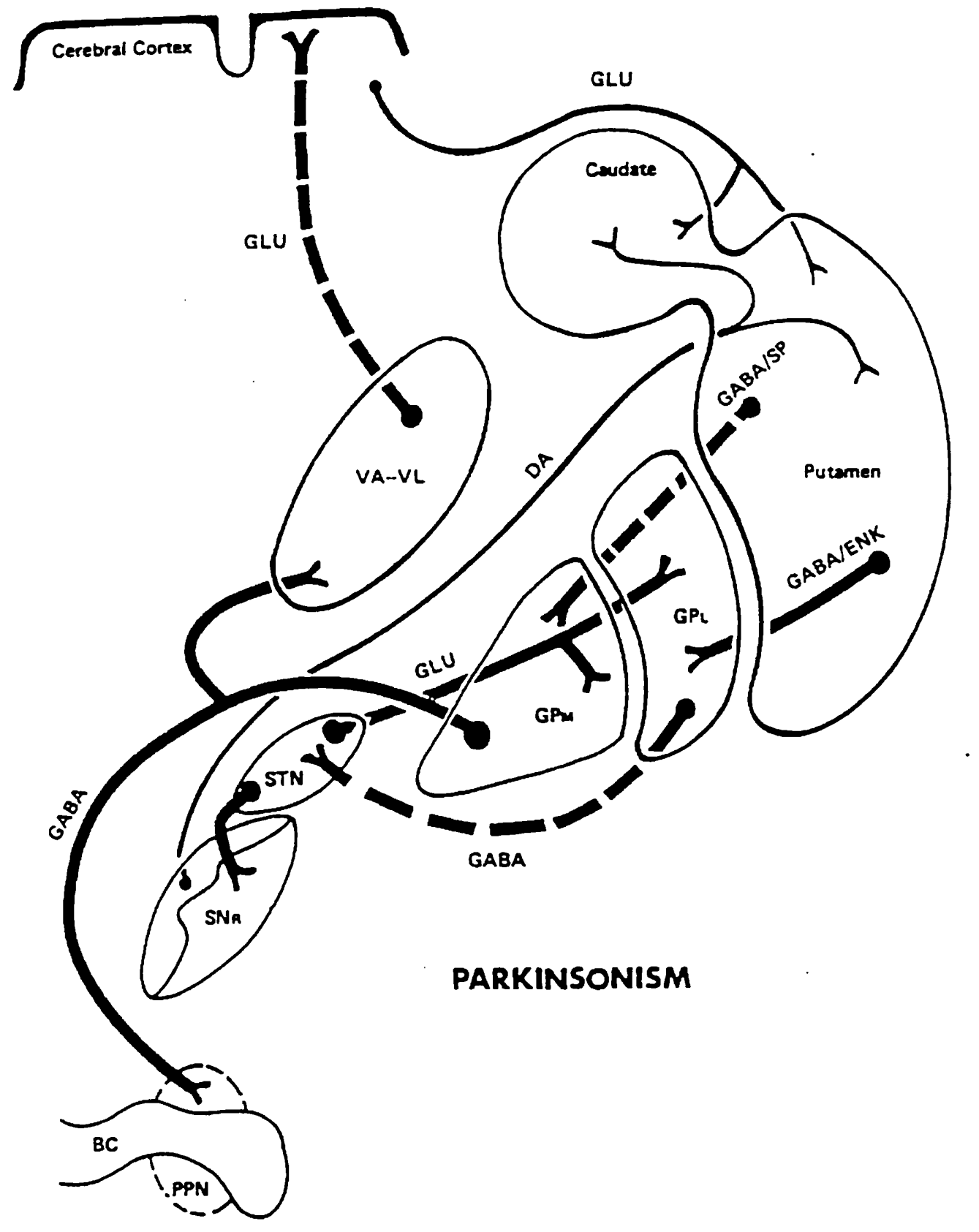


Tremor:That parkinsonian tremor is of central origin was suggested by the work of Pollock \& Davis (1930) (Pollock \& Davis 1930) in which deafferentation of a shaking limb relieved rigidity but not tremor. Different frequencies of tremor occur in the face, arm and leg muscles suggesting that the basic mechanisms of tremor lie in the brainstem (Hassler et al. 1979). However, with microelectrode recordings in the experimental primate rhythmic bursts of activity with a frequency of $4-5 \mathrm{~Hz}$, in phase with limb tremor have been recorded from the thalamic nucleus intermedius (VIM) and caudal nucleus ventralis oralis posterior (VOP), the motor cortex and the corticospinal tract. This persists after curarisation and deafferentation of the affected limbs (Cordeau 1961, Lamarre \& Cordeau 1962). These findings were confirmed in recordings in parkinsonian patients. These results suggest that a central tremorogenic pacemaker activates the motor cortex and thereby the spinal motor neurons via the corticospinal tract. Thus excessive tonic discharges are not the only physiological abnormality of the basal ganglia in PD, and increasing evidence indicates that phasic oscillations in neuronal firing are responsible for tremor. However, phasic firing in synchrony with tremor in the limbs can be recorded not only from the motor thalamus, the long thought of 'pacemaker', but also in STN, GPm and even in GPI. In parkinsonian monkeys and patients with PD, a lesion of GPm and particularly in STN may abolish tremor, indicating a role of the STN-GPm axis in tremorgenesis in PD.

Akinesia: Akinesia is probably the least well understood of the parkinsonian symptoms. The work of Tanji and Kurata (Tanji \& Kurata 1983) would suggest that the supplementary cortex, which receives a significant pallidal input, plays a preparatory role in initiating movement. The current consensus is that excessive pallidal inhibition secondary to dopamine depletion leads to a functional disconnection between the SMA and the basal ganglia. Most research has concentrated on the thalamic nuclei which are targeted by the 
ascending projections of the basal ganglia. Hence it is widely believed that parkinsonian akinesia results mainly from over inhibition of thalamo-cortical feedback loops by the overactive GPm.

However, this view fails to explain the clinical and experimental observation that thalamotomy, despite relieving tremor, rarely improves akinesia and may even result in a worsening of this symptom (Laitinen et al 1973). Conversely medial pallidotomy may alleviate akinesia but has a lesser effect on tremor (Baron et al 1996), whereas high frequency stimulation or lesioning of the STN improves both symptoms (Aziz et al 1991, Benazzouz et al 1996, Limousin et al 1995). As thalamic lesioning does not affect the descending outputs of the basal ganglia, whereas pallidotomy and subthalamic nucleotomy do, a logical conclusion would be that the overactivity of descending projections to the pedunculopontine area in the upper brainstem (Aziz et al 1992, Jackson et al 1983,) rather than overinhibition of the thalamic motor nuclei, is responsible for the akinesia of PD.

In support of this hypothesis the optimal target for relief of akinesia by medial pallidotomy is the posteroventral third of GPm, the region which has been previously demonstrated to project to PPN (Takada 1995). Also, post mortem studies have revealed that in PD there is a depletion of neurons in the PPN region (Zweig 1989). One possible explanation for this loss would be that it is secondary to the excessive inhibition of this cell group by the overactivity of the descendingpallidal projections. Furthermore, in rats electrical or pharmacological stimulation of the PPN has been shown to increase, whereas PPN inhibition decreases, the animal's locomotor activity" (Brudzynski et al 1986, Garcia-Rill 1987, Milner et al 1988, Mogenson et al 1988).

\section{CLINICAL FEATURES OF PARKINSON'S DISEASE}

The clinical features of Parkinson's disease are well recognized. Therefore, a brief review of the cardinal features alone will be discussed. TRAP (tremor, 
rigidity, akinesia and postural instability) constitute the cardinal symptoms of Parkinson's disease.

Tremor: The tremor of parkinsonism characteristically occurs when the affected limb is at rest and fully supported. It disappears with action and sleep and characteristically has a frequency of $4-5 \mathrm{cps}$. An arm is usually affected before the ipsilateral leg. When most pronounced in the hands and fingers it takes on the "pill-rolling" appearance.

Action tremor is not unusual in Parkinson's disease, in one study (Lance et al. 1963) it was present in 23 of 40 patients and was the only tremor affecting 6. It has a different frequency $(6-12 \mathrm{cps})$ from that of resting tremor. As will be discussed, tremor appears to be of central origin subsequent to excessive pallidothalamic inhibition.

Rigidity: Rigidity in Parkinson's disease is classically of the lead pipe or cog-wheel type most easily appreciated by slow extension-flexion movements about a joint. It was originally thought to be inextricably linked to akinesia but is now known not to be the case. Surgery can alleviate rigidity reliably without affecting akinesia and certain patients can be akinetic with normal muscle tone. The origins of the altered muscle tone are as yet unclear. An altered proprioceptive reflex may be part of the picture as selective deafferentation of affected limbs with local anaesthetic applied to peripheral nerves can reduce the rigidity. However, stretch reflexes are not usually enhanced as might be expected if this were the case. It is also possible that central mechanisms that induce tremor may also, if exaggerated, lead to generalized rigidity.

Akinesia: The most disabling feature of the condition is the inability to initiate spontaneous movement and slowness of its execution. All manner of movement is lost leaving the patient immobile, expressionless, not even blinking. Despite this crippling akinesia patients can exhibit sudden bursts of 
rapid normal movements given the right stimulus, hyperkinesis paradoxica. Although a patient knows how and what he wants to do it is as if there is an equal and opposite force preventing its initiation and execution (Sacks 1991). It is probable that the origins of akinesia lie in an imbalance between the direct and indirect cortico-pallidal interactions (Alexander \& Crutcher 1990). Normally cortical activity will drive the striatum to reduce pallidal inhibition of the thalamus so that execution of movement is facilitated but in PD the resting pallidal output is so high that this is no longer feasible. Evidence, including our own to be herein presented, would also suggest that upper brain stem mechanisms at the level of the pedunculopontine nucleus are essential in the generation of this most disabling symptom.

Postural instability: Another major problem encountered by PD patients is an impairment of the 'righting reflexes'. As a result patients will continue to move in the direction imposed by a shifting center of gravity. The cause for this may be partly vestibular in origin. In one study (Reichart et al. 1982) using caloric testing and electronystagmography $31 \%$ of PD patients had impaired and $31 \%$ absent responses. The result is that parkinsonian patients are subject to frequent falls and are least able to protect themselves.

Other symptoms: A number of lesser symptoms affecting parkinsonian patients are weakness of speech, dysphagia, delayed gastric emptying with early post-prandial satiation and discomfort, constipation, loss of appetite, urinary problems, impotence, excessive sweating, seborrhea and severe limb pain of unclear aetiology. Limb pain is exacerbated by, and may even be due to rigidity and dystonia.

\section{MEDICAL THERAPY OF PARKINSON'S DISEASE}

Parkinson's disease is unique in that the identification of a biochemical lesion, nigro-striatal dopamine depletion, has led to an effective medical therapy i.e. levo-dopa (Cotzias et al. 1969a,b, Duvoisin et al. 1969). The response of the 
condition to levo-dopa in combination with a DOPA decarboxylase inhibitor is such that failure to respond calls the diagnosis into question. The phenomenal success which followed the introduction of L-dopa therapy in the 1960's saw a corresponding dramatic decline in the popularity and practice of surgery for parkinsonism. Nevertheless, despite its' phenomenal and enduring success, levo-dopa therapy is not problem free as was to become apparent in the years subsequent to its' introduction. $80 \%$ of patients on high dose levodopa therapy for six years or more will experience dyskinesias, dystonias and on-off phenomena. These are particularly likely to occur in patients under 50 years of age at diagnosis (Lees 1986). Therefore, alternative therapies or adjuvant treatments to minimize L-dopa dosage have been sought.

These alternative drugs and therapeutic measures have been used to varying effect. Charcot initially introduced anticholinergic therapy, in the form of belladona, with some benefit. Further analysis revealed that in general anticholinergics reduce tremor, rigidity and drooling only, and such treatment is attended by side effects which limit it's use (Marsden 1969).

Another approach has been continuous intravenous drug infusions using the soluble methyl ester of levo-dopa in addition to oral therapy (Nutt et al. 1984, Quinn et al. 1982, Shoulson et al. 1975). Apomorphine infused subcutaneously is another option (Stibe et al. 1987). These do reduce off time and dyskinesias but are not practical without intense medical staff involvement.

Transdermal 4-propyl-9-hydroxynapthoxazine (PHNO) administration was promising but limited by toxicity. Bromocriptine administration may be a useful adjunct as it allows for some reduction in levo-dopa dosage (Hoehn \& Elton 1985, Lieberman et al. 1984). The use of MAO-B inhibitors has been shown to beneficially affect the course of the disease, hence the early administration of Selegeline is recommended in some centers in patients with mild symptoms at presentation (Birkmayer et al. 1985, MacMahon \& Bland 
1996), although debate continues as to the exact safety of its usage (Jellinger 1995, Lees AJ 1995, Maki-Ikola et al 1995, Silva et al 1995).

Sleep deprivation for 24 hours has useful effect on dyskinesias, dystonias and on-off phenomena but is impractical (Bertolucci et al. 1987). Low protein diets have been found to improve symptom control whilst on levo-dopa, presumably by improving enteral absorption (Pincus \& Barry 1987, Riley \& Lang 1988).

However, the search for the panacea of a dopamine agonist which produces an adequate therapeutic response at minimal dosage levels and is free of adverse side effects continues to this day. Current interest is centered primarily around adjuvant drug therapies aimed at minimizing the dose of L-dopa necessary to produce a therapeutic effect, and thereby aiming to prolong the treatment period prior to the development of L-dopa induced side effects. These include the catechol-O-methyltransferases (COMT) inhibitors which work to increase the bio-availability of oral doses of L-dopa. There were problems with the first generation of such drugs resulting in hepatic impairment resulting in their withdrawal in 1998, however, a new 'safer' second generation of COMT inhibitors is already on the market. Such drugs may be considered as effecting a "chemical pallidotomy". Other groups are researching sodium channel blocking drugs, which they propose will reduce the incidence of dyskinesias associated with L-dopa therapy (Imperato et al 1998). However, none of these lines of investigation appear likely to produce an all encompassing result in the near future; therefore, a resurgence of interest in surgical therapies has been prevalent in recent years.

\section{SURGICAL TREATMENT OF PARKINSON'S DISEASE}

The history of the surgical treatment of movement disorders, often focussed about PD, is a 100 year old tale of serendipity and folly which has passed through several phases of popularity and practice to reach the present day. In 
the 1930's and 1940's, when the number of patients with PD were swelled by cohorts with post-encephalitic disease and when medical therapy was poorly developed by modern standards, surgery was frequently resorted to in an attempt to alleviate patients' distressing and exhausting tremor. During this time, operations were devised which attacked all levels of the nervous system with varying degrees of success, until attention came to focus on the basal ganglia at the later end of this period. The practice of surgical treatment went into a steep decline in the 1970's and 1980's with the introduction of L-dopa. However, with the now well documented complications of long-term L-dopa therapy becoming apparent, coupled with our improved understanding of basal ganglia functioning and advances in medical imaging the surgical treatment of movement disorders has seen a great resurgence in our current decade. Three phases in its' history can be identified;

(1) The Pyramidal Era.

(2) The Basal Ganglia and Thalamic Era.

(3) The Contemporary/Stereotactic Era.

Since James Parkinsons' original work in 1817, it has been recognized that a stroke is attended by loss of contralateral tremor. Extending this observation Horsley in 1890 performed excision of the motor cortex in a case of athetosis with good effect (Horsley 1909). However, his attempts were not followed up for several years. Neurosurgical attack on the pyramidal tract originated with such operations devised to resect either the premotor cortex, the motor cortex itself, or both. This cortical topectomy was championed by Klemme and Bucy, who both claimed to have been the first to introduce the technique despite Horsleys' reported case of 1890. Klemme's 1940 (Klemme 1940) report describes 100 resections of the premotor cortex performed, 39 achieved relief of tremor with no significant neurological deficits. However, he also reported a mortality of $17 \%$ for this procedure. While the scanty reports of Klemme indicated some value of the treatment, the detailed analysis of Bucy's series of 
patients who underwent resection of the premotor and motor cortex indicated that this aggressive resection left the patients initially hemiplegic which subsequently lessened in severity, leaving the patient with hemiparesis but without tremor (Bucy \& Case 1939). He subsequently employed the procedure only rarely for palliation in severe tremor-dominant patients. His observations led him to believe that only those procedures designed to interrupt the premotor area or pyramidal tract would abolish tremor. His conclusion stimulated others to interrupt the pyramidal tract below the cortex. Based upon this work, Putnam devised the operation of pyramidotomy which consisted of incising the pyramidal tract at the level of the second cervical segment of the spinal cord (Putnam 1938). This operation obviously produced an ipsilateral hemiplegia with attendant relief of tremor. The degree of lasting tremor relief was proportional to the sacrifice of the motor function. One advantage of this procedure over the cortical topectomies performed earlier was it avoided the convulsive side effects noted with the cranial procedures. Subsequently, Walker introduced the pedunculotomy to interrupt pyramidal fibers at the level of the midbrain (Walker 1952). Similar to the observations of Putnam, the control of tremor was inversely related to the residual motor function following section of the peduncle. A compromise between paralysis and freedom from tremor was the best that could be expected from attacking the pyramidal tract surgically, whether at its' origin in the cortex, in the cerebral peduncle, or within the spinal cord. Other early procedures, which were discarded due to limited success, included; cervical dorsal rhizotomy (Foester 1927, Leriche 1912, Pollock \& Davis 1930, Putnam 1938), cord section (Ebin 1949, Oliver 1950, Putnam 1940) and cerebellar dentatectomy (DelmasMarsalet \& Bogaert 1935). Thus, surgical experience with operative interventions which extended into the early 1950's aimed at the pyramidal tract were performed solely for tremor, and at the expense of motor function. 
None of the other manifestations of PD (akinesia, rigidity) were improved by any of the techniques performed during the 'Pyramidal era'.

Meyer's investigations into the surgery of the basal ganglia in the 1940's constituted a major advance in the surgical therapy of parkinsonism focusing attention on the basal ganglia (Meyers 1940,1942,1951). His work is a tribute to the courage of his patients and an indication of their desperate need for help! His first attempt was to resect the head of the caudate nucleus, but this failed to regularly alleviate tremor and rigidity. Subsequently he experimented with; a.)Resection of the head of the caudate nucleus and interruption of fibers in the oral portion of the anterior limb of the internal capsule.

b.)Removal of the head of caudate with the oral $1 / 3$ of the globus pallidus and putamen.

c.) Section of the pallidofugal fibers from the medial globus pallidum.

He noted that the best improvement in both tremor and rigidity was with pallidofugal section - the so-called Meyer's procedure. He utilized a cortical incision and transventricular approach, reporting a 60\% improvement in rigidity and tremor with a mortality rate of $15.7 \%$. However, there was associated with Meyer's procedure a significant incidence of postoperative convulsions and patients were left disabled by memory deficits and epilepsy. Browder modified Meyer's approach to remove the upper third of the head of caudate, and sectioned the fibers of the anterior limb of the internal capsule until a paresis of the hand resulted (Browder 1948). The adjacent regions of the putamen and pallidum were removed, and he noted a significant reduction of the tremor and rigidity of the contralateral extremities. Browder's work although attended by a much higher mortality led to the same conclusion: that surgery should be aimed at the pallidofugal fibers and the ansa lenticularis. The next step forward in the surgical treatment of movement disorders again came as a matter of chance. Cooper reported the case of a 39 year old male with postencephalitic tremor and rigidity of the right extremities and severe 
retrocollis in whom he attempted a left cerebral pedunculotomy. The pedunculotomy was abandoned because of bleeding which was controlled by clipping the anterior choroidal artery. The patient survived, and to Cooper's surprise his postoperative course was distinguished by the absence of tremor and rigidity of the right extremities although their motor and sensory functions remained intact (Cooper1953). A postoperative angiogram confirmed the occlusion of the anterior choroidal artery. He subsequently purposefully cauterized the anterior choroidal artery in over 50 cases in the ensuing 3 years. He reported excellent results using this technique in $65 \%$ of cases, including improvements in tremor, rigidity, bradykinesia and gait disturbances. Neurovascular occlusion studies in primates and autopsy studies in humans had demonstrated that the medial and intermediate segments of the globus pallidus were irrigated by the anterior choroidal artery. Cooper suggested that in elderly patients with atherosclerotic disease the irrigation pattern of the anterior choroidal artery was variable, and may include regions such as the posterior limb of the internal capsule, and for this reason believed that hemiparesis was a more common complication of this procedure in such patients. He therefore suggested that the operation be restricted to patients under the age of 55. Shortly following this Cooper established that chemopallidectomy was equally effective (Cooper 1954). After direct injection of procaine within the globus pallidus as a test lesion, absolute alcohol was injected to create a permanent ablation if the procaine injection had been successful in alleviating symptoms. He preferred this alternative to anterior choroidal artery ligation in the elderly. Cooper's findings with chemopallidotomy were confirmed by others (Guiot \& Brion 1953, Narabayashi et al 1953,1956). The early experience with pallidotomy demonstrated that pallidotomy was best for the treatment of rigidity and somewhat inconsistent in the treatment of tremor (Fig.1.2). 
Figure 1.2. Early surgical targets in Parkinson's Disease

(Adapted from Cooper 1956)

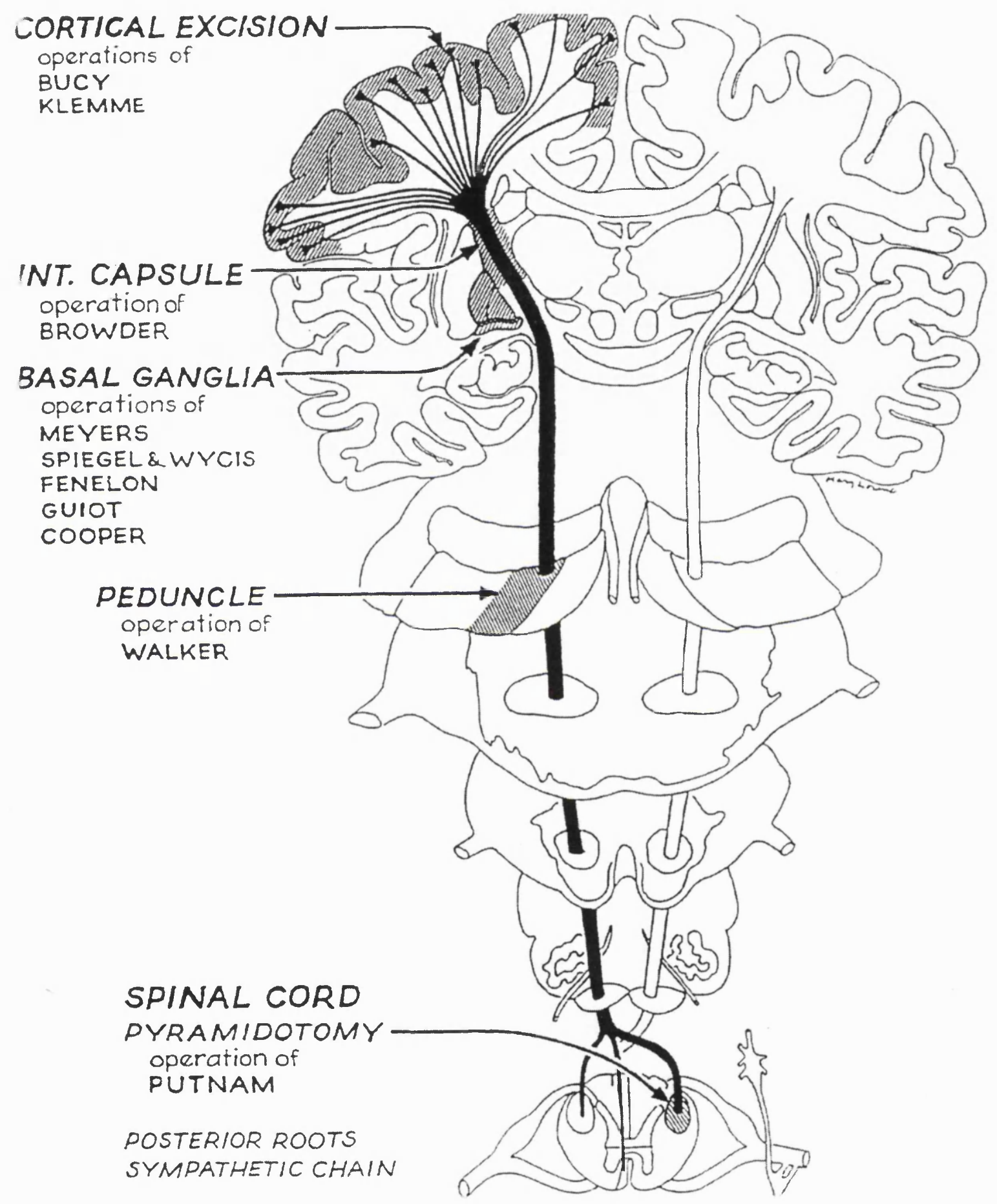


In 1950 Hassler determined that the output of the medial pallidum was predominantly to the ventroanterior nuclei of the thalamus (Hassler 1954,1958). Immediately Cooper and Bravo, and Hassler and Reichert, introduced an alternative surgical target, based upon the premise that if pallidal lesions were effective, then lesions in the target of their thalamic projections would also be effective. Thalamotomy proved to be more successful at alleviating tremor in most surgeon's experience, and, along with interruption of the pallidothalamic fibers in the subthalamus, rapidly became the prime operation for the relief of tremor in $\mathrm{PD}$ and other movement disorders. Although these early procedures were performed free-hand the scene was set for modern stereotactic neurosurgery.

By the 1950's a small area of the basal ganglia was defined as the optimal targets in parkinsonian surgery, comprising the medial pallidum and the ansa lenticularis for the treatment of rigidity, and the ventral thalamus for alleviation of tremor. However, the advent of L-dopa therapy was to result in a precipitous decline in the number of stereotactic procedures performed to alleviate movement disorders. There were those who persevered in their attempts to further refine lesional surgery for PD, and in 1960 Svennilson and Leksell published their results of a series of patients in whom they had performed posteroventral pallidotomy (Svennilson et al 1960). They noted that 19 out of 20 patients achieved sustained improvement in rigidity, tremor and bradykinesia. Perhaps due to the widespread interest in pharmacological therapy for the management of PD that shortly followed the publication of the article, combined with its' appearance in a Scandinavian journal with limited readership, there was little international recognition of the benefits of the operation. However, following nearly 30 years of experience with medical therapy, severe limitations to its' usage have become apparent. The effectiveness of L-dopa usually wanes within several years of treatment, and the drug may be associated with highly disabling side effects in many patients 
(dyskinesias, on/off fluctuations, psychosis, and gastrointestinal complaints). Therefore, there has in recent years been a resurgence of interest in surgical therapy for movement disorders. This interest was further fuelled by Laitinen's report of his results with Leksell's operation in a series of primarily hypokinetic patients who had failed on or not tolerated L-dopa therapy. He reported that in 37 out of 38 patients operated upon there was post operatively 'almost complete relief of rigidity and hypokinesia'(Laitinen 1992).

The principal issues which needed to be addressed in our 'contemporary era' of surgical treatment for movement disorders were; a method of accurate lesion placement with minimal attendant morbidity, optimum lesioning technique, optimum target site and the possibilities of any further therapies. At present several of these issues remain dubious. However, I will now outline their history and current controversies.

Since the late nineteenth century methods have been available, at least in concept, for reaching targets within the basal ganglia without causing damage along the approach. In 1861 Harting of Utrecht, is quoted (Iizuka 1975) as having described the "kephalograph" to make directed intracranial lesions in the experimental animal. Kandel (Kandel 1989) describes the work of Zernov in 1889 in which the development of an encephalometer for use in directed human surgery was reported. This used a polar system of co-ordinates and was successfully used to treat superficial brain lesions. However, this work failed to make any ingress into clinical practice generally.

Horsley and Clarke (Clarke \& Horsley 1908) described their studies of the cerebellar nuclei and the problems of performing deep lesions with minimal damage. To do so they began work on an accurate localization of intracranial structures in relation to external bony landmarks in the rhesus monkey. To this end they sub-divided the brain into 8 subdivisions by three planes. The frontal plane passed perpendicularly through the external auditory meati, the horizontal plane through the nasion and the coronal plane perpendicular to 
both. Each plane was subdivided into square millimeters. Clarke then sectioned frozen heads every $2 \mathrm{~mm}$., placed the sections under a glass plate ruled in $1 \mathrm{~mm}$. squares and photographed them, thus producing a topographical brain map. To allow for variation in size, a variable grid with adjustable crossed silk threads was devised. With an accurate map to hand Clarke then devised a frame that could be fixed to the skull with an adjustable needle holder that could pass a needle to any selected target. He went on to develop a more advanced frame using an equatorial system. A circular turntable fixed to the skull consisting of two flat rings capable of a $360^{\circ}$ rotation supported a semi-circular arc carrying the needle holder. This allowed the approach of the target from any inclination. Subsequently he fell out with Horsley over credit for the work done and went into general practice having spent much of his personal savings in the development of the frame!

Forty years later extending the work of Horsley and Clarke, Spiegel and Wycis developed the tools to reach the target with minimal surgery and maximum accuracy with their introduction of the stereo-encephalotome to surgery (Spiegel et al. 1947). Their original work evolved out of a concern to produce precise intracerebral lesions for psychosurgery and the relief of pain.

The original frame was fixed to the head with a plaster cast but later models used rigid fixation to the skull with screws (Fig. 1.3).

The principle underlying stereo-tactic neurosurgery is that elucidated by Horsley and Clarke. Any point within an irregular body (the skull) can be located in three dimensions if it is rigidly fixed in space enclosed by a rectilinear frame. Initially the positions of targets of interest were calculated within the frame from skeletal landmarks. These however, are too variable and atlases of stereotaxy began to use internal X-ray identified landmarks such as the AC-PC (Anterior commisure - Posterior commisure) or FM-PC (Foramen of Monroe - posterior commisure) line, positions of which could be 
Figure 1.3. Stereoencephalotome of Spiegel and Wycis.

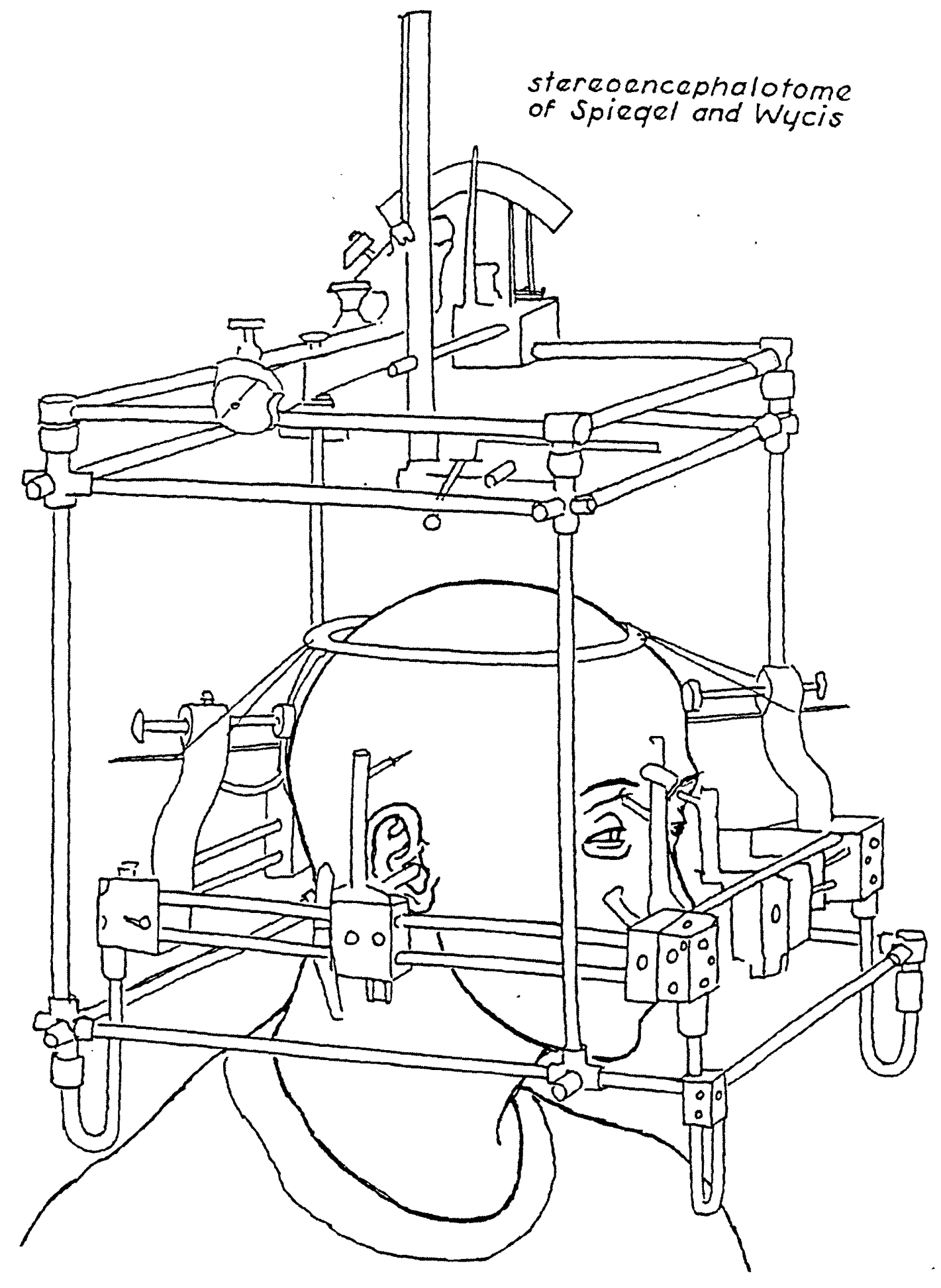


determined from contrast ventriculography done with the patient's head fixed in the frame. This remains the choice method today in centers utilizing ventriculography for targeting, though calculation of co-ordinates using angular rather than Cartesian frames of reference have also gained popularity. A persistent problem with the use of ventriculography in conjunction with atlases has been the inherent anatomical variability in the position of target structures in relation to fixed landmarks. The study of Van Buren (Van Buren \& McCubbin 1962) illustrates the degree of variability that can exist between similarly sized brains. A common procedure to allow for this is the "relativity factor" of Mundinger and Reichert in which the co-ordinates for a target in an individual patient are multiplied by the ratio of the patient's measured AC-PC length to that of the atlas used.

With the development of new technologies alternative methods of localization have become possible, in particular the use of CT and or MRI for target localization in stereotactic surgery. With the advent of CT and MRI compatible frames it is now possible to scan a patient with the frame attached and acquire target co-ordinates without recourse to ventriculography. Satisfactory results have repeatedly been confirmed with image guided techniques over the last decade (Aziz \& Torrens 1989, Baron et al 1996, Laitinen 1985, Leksell et al. 1985, Uematsu \& Rosenbaum 1987). Current technology allows for the "fusion" of an MRI to a CT image and attendant spatial correction (Papanastassiou et al 19983), this targeting technique reports good clinical outcomes with a major complication rate of only $3 \%$ (Rowe et al 1999). Furthermore, microelectrode extra-cellular recording (MER) of unit activity has been repeatedly described as a reliable method to ensure accurate electrode placement based on radiologically derived co-ordinates (Baron et al 1996, Kopyov 1997, Krauss 1997, Lozano 1996). However, studies published to date show that MER, as compared to image-guided or macrostimulation alone, may be associated with a higher rate of complications (Carroll et al 
1998). Macro-stimulation is another method of affirming accurate targeting, in that stimulation of the target site at low frequencies $(2 \mathrm{~Hz})$ causes exacerbation of tremor and rigidity and inhibition of the same when using high frequencies $(100 \mathrm{~Hz})$ if the electrode is correctly located. Consequently recording and/or stimulation to check electrode placement has become standard practice for performing lesioning surgery for movement disorders. Thus, almost all functional neurosurgery is carried out under local anaesthesia so that effects of stimulation can be monitored to avoid untoward irreversible results.

Over the years a variety of techniques were used to create lesions. Mechanical methods using leucotomes or wire loops were popular, but these were attended by a high risk of hemorrhage. Chemical lesions using alcohol were too toxic though a procaine-oil-wax mixture claimed better results. A combination of mechanical and chemical methods was to insert an inflatable balloon to create a cavity into which alcohol could be instilled (Cooper \& Bravo 1958). This was also attended by unpredictable hemorrhage however. Low temperature techniques were popularized by Cooper (Cooper et al. 1965) and said to be free of risk of hemorrhage and reversible lesions could be made to check placement accuracy. However, this technique was not as safe as initially thought, and the lesions were variable in size. Induction heating of stereotactically implanted metal pellets by placing the patients head in a large coil carrying a high frequency current (Walker \& Burton 1966) was tried but problems of pellet migration led to this being abandoned. Meyers (Meyers et al 1959) developed the use of focussed ultrasound to create lesions but this technique required a craniotomy, complicated apparatus; and it prolonged operating time immensely. Radioactive pellets and external beam irradiation (Leksell et al. 1960) have been used but the lesion size is variable and with radiation arteritis the lesions may continue to expand beyond optimal. However, there remain advocates of such techniques who today utilize cobalt powered Gamma Knife Units, as described by Leksell and Larsson in the 
1950's, to create stereotactic lesions in the thalamus principally (Rand et al 1995). Electrical methods using bipolar and monopolar electrodes still have their adherents but the most widely practiced technique today (Gildenberg 1975) uses high, radio frequency coagulation. In this technique passing a pure sine wave current of $100,000-500,000 \mathrm{~Hz}$ from the uninsulated tip of a stereotactically placed electrode causes ionic agitation of the tissue around it, rapidly raising its temperature. Monitoring the current, tip temperature and duration of radio frequency allows for predictable and safe lesions. Present generators allow monitoring of electrical activity and impedance of tissue through the same electrode which is a valuable adjunct to increase accuracy of placement. This is now so widely used as to be virtually standard (Favre et al 1996).

An alternative to lesioning that has evolved over recent years has been the use of Deep Brain Stimulators. These are chronically acting, implantable devices which are targeted for insertion in exactly the same stereotactic manner as a lesion would be. They contain at their distal extremity a variable number of contact electrodes through which a current of variable character may be passed. They may be adjusted and controlled by the patients themselves via a magnetic handset communicating with a proximal control box usually implanted in the subclavian region Although the exact mechanism of deep brain stimulation is unknown, recent evidence suggests that it, in part, functions to restore normal firing patterns of neurons that are altered in PD (Boraud et al 1996). Reported results using such stimulators have been comparable to lesion studies at all sites investigated to date (Benabid et al 1989, 1996, Krack et al 1998, Limousin et al 1995, Tronnier et al 1997).

The next question arising is what is the most effective and safest site for performing a lesion or, implanting a stimulator, in order to relieve the symptoms of PD and other movement disorders? At the advent of our contemporary/stereotactic era attention had been focussed on to the 
ventrolateral thalamus, and the posteroventral pallidum, as previously described (Fig. 1.4). Interest has remained centered largely around these areas in the last decade with multiple series investigating the functional outcomes of such procedures now being performed by the refined techniques outlined earlier. Pallidotomy and thalamotomy are now considered as 'safe' procedures with an incidence of major, symptomatic and perioperative complications of only $1-3 \%$. The choice of procedure is now focussed toward the symptomatology being treated. Thalamotomy or thalamic stimulation is widely reserved for the relief of distal tremor (Benabid et al 1996, Burchiel 1995, Goldman et al 1992, Jankovic et al 1995, Koller et al 1997, Tasker et al 1997), whereas posteroventral pallidotomy (PVP) or GPm stimulation is the procedure of choice in treating dyskinesias and bradykinesias (Bejjani et al 1998, Krack et al 1998, Lozano \& Lang 1998, Pahwa et al 1997, Seigfried \& Lippitz 1994). Careful lesion placement within the bounds of GPm is emphasised by case reports of an exacerbation of PD symptoms with lesions encroaching upon the GPl (Munro-Davies et al 1999).

Current controversy reigns over the safety of performing bilateral lesions in the symmetrically affected patient. Historically bilateral thalamotomy was associated with a high incidence of bulbar deficits that prevented its routine use (Cooper 1965). Also $48.2 \%$ of patients that had undergone a pallidotomy followed by a contralateral thalamotomy were reported to suffer complications such as aphonia, decreased attention and dysarthria (Krayenbuhl et al 1961). High morbidity previously reported with bilateral PVP may relate to large bilateral lesions resulting in cognitive impairment, and often hypophonia (Galvez-Jimenez et al 1996). Recently however, several centers have safely performed both sequential and simultaneous bilateral PVP (Iacono et al 1995, Laitinen et al 1992, Shima et al 1996, Schuuman et al 1997). Only one small study has directly compared unilateral and bilateral PVP confirming an enhanced outcome in measured scores in bilateral PVP. However, axial 
Figure 1.4. Leksell's Posteroventral Pallidotomy Target - L Classical Posteroventral Pallidotomy Target -A (Taken from Laitinen et al 1992)
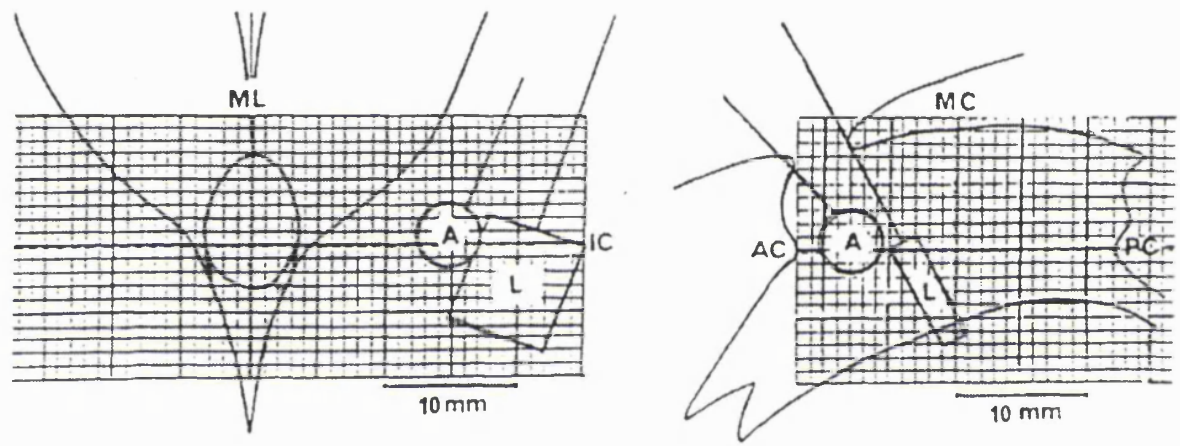
symptoms may worsen and dysarthria and dysphonia may arise (Scott et al 1998). Undoubtedly better targeting and lesion making in recent years lead to lower rates of complications. Nevertheless bilateral lesions are not widely performed and current thinking would lean towards the insertion of bilateral stimulators in the symmetrically affected patient.

A third site for both lesional and stimulator surgery has gained popularity and support in more recent years, that being the subthalamic nucleus. Subthalamotomies were first described as effective lesions by Andy and Hughes (Andy et al 1963, Hughes 1969). Such lesions were targeted at the fields of Forel and the ansa lenticularis carefully avoiding the subthalamic nucleus itself. This avoided the danger of post-operative development of hemiballism thought at the time to be potentially fatal (Meyers et al 1950, Whittier 1947). However in a comparison of subthalamotomy to VL thalamotomy Fager (Fager 1968) found the latter to be more reliable. Subsequently it became an infrequently performed procedure. As the understanding of basal ganglia physiology and movement control was further elucidated, largely through the work in MPTP treated monkeys, it was shown that the subthalamic nucleus has a pivotal role to play (DeLong et al 1984, DeLong 1990) and interest in it as a surgical target was renewed. Lesions of the STN in MPTP monkeys induced a dramatic improvement in the parkinsonian triad (Aziz et al 1991, Bergmann et al 1990, Guridi et al 1996). This was shown to be replicated by stimulation techniques in monkeys (Benazzouz et al 1993) and in patients' with PD (Limousin et al 1995, Pollack et al 1998). Also, stimulation has been shown to be effective against not only the cardinal features of PD, but also against drug induced dyskinesias (Krack et al 1997). Reluctance persists against lesioning at this site due to the theoretical risks of hemiballismus and hemorrhage, neither of which are common after stimulation. However a few centers are now performing STN 
lesions in PD patients and early results are encouraging (Gill \& Heywood 1997).

An alternative surgical approach to treating PD is that of cell transplantation. Transplantation of CNS tissue is one hundred years', old beginning with the work of G. Thompson in 1890. Dunn in 1917 reported better results with grafts of immature rat brain tissue to adult rats, and in 1940 Le Gros Clark repeated the work in fetal to neonatal rats. The work lay relatively dormant until the 1970's with the work of Bjorklund, Das and others. They found that experimental parkinsonism could be alleviated by intracerebral fetal dopaminergic cell grafts (Lindvall \& Bjorklund 1979, Lindvall 1989). This led to human grafts of autologous adrenal medullary tissue with the initially phenomenal reports reputed by Madrazo in 1987 (Madrazo et al. 1987). Subsequent to this, a further 100 cases were reported mostly of caudate and putaminal implants of fetal mesencephalic tissue. The reports of any improvement (Lindvall et al. 1989) are marginal and fail to reach significant levels though there were still some who report marked improvement (LopezLozano et al. 1991). The controversy surrounding the acquisition of fetal graft tissue, and the limited quantities in which such tissue is available, led to the research and development of xenographic cell lines for transplantation. As with previous series of transplanted patients results have been variable and numbers limited. One group however, have been able to provide histological evidence of long term graft survival using fetal porcine implants (Deacon et al 1997) in the light of positive clinical results (Elias et al 1998). This work does imply a future for the technique, but much more experimental animal and surgical work is needed before a final appraisal. 


\section{ANATOMY OF THE PEDUNCULOPONTINE NUCLEUS}

The PPN consists of a neurochemically and morphologically heterogenous population of neurons. In the human brain, the PPN is bounded on its' lateral side by fibers of the medial lemniscus and on its' medial side by fibers of the superior cerebellar peduncle and its' decussation (Geula et al 1993, Olszewski\&Baxter 1982). Rostrally, the anterior aspect of the PPN contacts the dorso-medial aspect of the posterolateral $\mathrm{SN}$, while the retrorurbal field borders it dorsally. Caudally, the most dorsal aspect of the PPN is limited by the cuneiform and subcuneiform nuclei and ventrally by the pontine reticular formation. The most caudal pole of the PPN is adjacent to neurons of the locus ceruleus.

Two subdivisions of the PPN have been characterized on the basis of cell density. The largely cholinergic pars compacta of the PPN (PPNc) is located within the caudal half of the nucleus in its dorsolateral aspect. Cells of the subnucleus pars dissipatus (PPNd) are distributed sparsely within the superior cerebellar peduncle and central tegmental tract. The pars compacta and dissipata have been described in humans, monkeys and subprimates (Geula et al 1993, Lavoie\&Parent 1994a, Mesulam et al 1983). The cholinergic neurons lie mainly within the PPNc in humans. Their number has been estimated at between 10,000 and 15,000 (Garcia-Rill et al 1995a,b). Cholinergic PPNc neurons are clustered along the dorsolateral border of the superior cerebellar peduncle (SP) at the level of the trochlear nuclei, whereas those in the PPNd are scattered along the SP from midmesencephalic to midpontine levels. In the human brainstem the cholinergic neuronal population of the PPN constitutes more than $90 \%$ of the neuronal population of the PPNc, whereas this percentage varies from $25-75 \%$ in the PPNd (Mesulam et al 1989).

A second prominent neuronal population contained within the traditional boundaries of the human and non-human PPNd is glutamatergic (Lavoie\&Parent 1994a, Rye et al 1995b/1996,), Lavoie and Parent 
(Lavoie\&Parent 1994a) found that at the level of the trochlear nucleus about $40 \%$ of monkey PPN cells expressed cholinergic and glutamatergic immunoreactivity. Additional neuronal types contained within the traditional boundaries of the non-human PPN include a dopaminergic population (Rye et al 1987), a noradrenergic group and a small group of GABAergic interneurons (Jones 1991).

Afferents of the PPN : Very little is known about the inputs and outputs of the human PPN. Anatomical and electrophysiological studies on nonhumans have identified many putative afferents to the mesopontine tegmental region containing the PPN, but because it is neurochemically heterogenous and closely apposed to several other functionally distinct regions and fiber tracts, it has been difficult to determine precisely which of these afferents actually terminate in the region, and of these, which terminate on cholinergic as opposed to non-cholinergic neurons.

Afferents from GPm and SNr are the most widely studied and established connections to the PPN (Shink et al 1997, Spann\&Grofova 1991). Recent studies on humans and other primates have shown that these projections appear to terminate preferentially on the non-cholinergic cells of the PPNd and largely avoid the cholinergic neurons of the PPNc (Rye et al 1995, Shink et al 1997, Spann \& Grofova 1991). These are probably inhibitory GABAergic efferents onto glutamatergic PPN neurons (Granata \& Kitai 1991, Noda \& Oka 1986,). Furthermore, other studies have shown that $80 \%$ of GPm neurons that project to the PPN send axon collaterals to the ventrolateral nucleus of the thalamus (Harnois \& Filion 1982). Inputs from the STN have been strongly suggested but have not been firmly established (Granata\&Kitai 1991, Hammond et al 1983, Jackson\&Crossman 1983, Kita\&Kitai 1987, Steininger et al 1992).

A number of other putative afferents to the PPN in several different species have been proposed arising from the limbic system (amygdala, hypothalamus, 
zona incerta, ascending reticular activating system (raphe nuclei, locus coerulus, laterodorsal tegmentum, contralateral PPN), as well as from premotor and supplementary motor areas, SNc, caudate, putamen, superior colliculus, basal forebrain, deep cerebellar nuclei, cervical and lumbar expansions of the spinal cord (Winn et al 1997). In general, the PPN receives bilateral inputs but with a preponderance of ipsilateral connections.

Efferents from the PPN : The outputs of the PPN can be divided into descending and ascending parts, with cholinergic and non-cholinergic neurons contributing to both. Although some PPN neurons have specific ascending projections and others specific descending projections, some collateralise and project in both directions (Spann\&Grofova 1991). The ascending projections of the PPN are far more prominent than those descending.

Ascending Projections. Ascending PPN outputs project via the dorsal and ventral tegmental bundles, with the dorsal tegmental pathway carrying the major cholinergic projection (Garcia-Rill 1991). The majority of ascending cholinergic PPN neurons are thought to project to all thalamic nuclei in the rat (Hallanger et al 1987, Rye et al 1987) cat (Steriade et al 1988) and monkey (Lavoie \& Parent 1994b). It has been estimated that $60 \%$ of PPN neurons project to the thalamus and that $90 \%$ of these are cholinergic in the rat (Sofroniew et al 1985).

Substantia Nigra (SN).Ascending projections provide massive innervation to the basal ganglia via the ventral tegmental bundle (Lavoie \& Parent 1994a-c). Neurons located in the center of the PPN (at the level of the trochlear nuclei) are those that provide the maximal innervation of the basal ganglia. Labeling with anterograde tracers have shown that SNc and the STN are by far the most densely innervated basal ganglia structures. PPN innervation of the basal ganglia in primates and non-primates is bilaterally organized. Approximately $40 \%$ of PPN neurons project contralaterally to the basal ganglia in primates (Lavoie\&Parent 1994b). PPN efferents reach their target sites in the basal 
ganglia by ascending along the lenticular fasiculus and ansa lenticularis, which are the main output tracts of the GPm .The PPN sends monosynaptic excitatory cholinergic projections to the dopamine containing neurons of the SNc with a minor cholinergic contingent to the SNr (Blaha \& Winn 1993, Chapman et al 1997, Scarnati et al 1988, Woolf\&Butcher 1986). However, most pedunculonigral fibers originate in the PPNd, and only a few originate in the PPNc in the primate and rat (Lavoie \& Parent 1994b, Spann \& Grofova 1991). The proportion of cholinergic neurons of the PPN that project to the $\mathrm{SN}$ is about $40 \%$ in the rat and $25 \%$ in the monkey (Gould et al 1989, Lavoie \& Parent 1994c).

PPNd glutamatergic neurons form excitatory synaptic contacts with dopaminergic neurons in the SNc in the non-human primate and rats (Charara et al 1996, Takakusaki et al 1996). Single PPN terminals in contact with SNc dopaminergic neurons may contain both glutamate and acetylcholine (Charara et al 1996, Lavoie \& Parent 1994a). Furthermore, the PPN may be a source of GABAergic efferents to the SNc in primates (Charara et al 1996).

Subthalamic Nucleus (STN). The existence of a massive and bilateral projection from the PPN to the STN has been documented in a number of species including the rat (Woolf\&Butcher 1986), cat (Moon-Edley et al 1983), and monkey (Lavoie \& Parent 1994b). The exact chemical nature of these PPN efferents has not yet been established although, in the rat, the majority of neurons are non-cholinergic (Lee et al 1988). Further evidence in rats suggests that PPN efferents to the STN and adjacent zona incerta are collaterals of PPN efferents to the pallidal complex )Hammond et al 1983, Hallanger \& Wainer 1988). Electrophysiological studies in the rat have suggested that the ascending projection from the PPN to the STN is excitatory (Hammond et al 1983).

Globus Pallidum (GP). In the monkey the PPN innervation of the pallidal complex is much less massive than that of the STN and SN. The 
pedunculopallidal fibers aborize more profusely in the monkey GPm than in the GPl (Lavoie \& Parent 1994b). A similar preference for the GPm exists in the rat and cat. The neurotransmitter used by pedunculopallidal neurons has yet to be established with certainty. In the human brain the GP receives cholinergic innervation the majority of which is thought to originate from brainstem cholinergic nuclei (Mesulam et al 1983). Other sites include the superior colliculus and a number of limbic structures (basal forebrain, hypothalamus, zona incerta, and amygdala). These ascending connections have been recently reviewed (Inglis\&Winn 1997, Reese et al 1995).

Descending Projections. Descending targets include several midbrain, pontine and medullary areas including several nuclei of the reticular formation, as well as deep cerebellar nuclei and the spinal cord as shown in the rat, cat and monkey (reviewed by; Reese et al 1995, Steininger 1997) but not yet demonstrated in human. These descending projections are thought to collateralise extensively (Rye et al 1988). The direct projection from the PPN to the spinal cord is thought to be non-cholinergic (Goldsmith \& Van der Kooy 1988, Shirommani et al 1988a,b, Skinner et al 1990, Sofroniew et al 1985), although some cholinergic neurons have been shown to project to the spinal cord (Rye et al 1988). Some of these descending projections are thought to terminate on the interneurons in lamina VII on both sides of the spinal cord rather than directly on motor neurons. However, most of the descending axons travel a shorter distance to the medullary reticular formation, which in turn provides bilateral outputs to the more laterally placed lamina VII interneurons in the spinal cord.

In general, all of these extensive ascending and descending projections of the PPN have been described in the rat, cat and monkey thus the connections of this system appear well preserved throughout these three species. However, minimal confirmed data is available in humans. 


\section{THE ROLE OF THE PPN IN LOCOMOTION}

The PPN has been linked to the so-called mesencephalic locomotor region (MLR), a functionally defined area of the brainstem within which it is possible to elicit controlled locomotion (i.e. locomotion in which increasingly higher levels of current drive the frequency of a stepping from a walk to a trot to a gallop) on a treadmill in the decerebrate rat (Garcia-Rill \& Skinner 1987a,b) and cat (Garcia-Rill 1986) and possibly, though in a less well defined manner in the monkey (Eidelberg et al 1981). Although the locations of optimal locomotion induction sites are within the cholinergic neurons of the PPNc (Garcia-Rill et al 1987a,b), there are several different brainstem regions, including prominent sensory nuclei, which can be stimulated to recruit locomotion (reviewed by Reese et al 1995). Each of these areas possesses direct outputs to the spine (the site of putative locomotor generators) and none of these areas can be considered to be specific for locomotion to the exclusion of other functions.

During locomotion the brain recruits a dedicated neural network. This control system is designed similarly in all classes of vertebrates (Grillner 1985), as might be expected from an evolutionary perspective. Thus, the same mesopontine and diencephalic centers initiate locomotion in lamphreys and primates, through the activation of lower brainstem reticulospinal neurons. These, in turn, activate spinal networks of neurons which generate the motor pattern. Sensory feedback is an integral part of the control system and helps adapt the motor pattern to external events. This sensory input is important for initiation of movements and for providing ongoing feedback for movement maintenance. Details of the cellular and molecular function of this network is now being elucidated in simple systems models as in the lamphey (Viana De Prisco et al 1997).

The responsiveness of PPN neurons to somatosensory stimuli (Grunwerg et al 1992, Reese et al 1995) and the projections from the lamina I of the spinal 
cord to the area of the PPN (Hylden et al 1985) suggests that the PPN may also be implicated in the control of sensory modulation, probably relaying sensory feedback to the thalamic nuclei. The role of the PPN as a feedback relay station is important for posture, gait initiation and modulation and would not be unexpected given its' more prominent ascending projections (notably cholinergic inputs to the thalamus) and its' connections with deep cerebellar nuclei.

Three separate populations of neurons displaying rhythmic activity in relation to locomotion can be recorded extracellularly in the region of the PPN in the decerebrate cat (Garcia-Rill \& Skinner 1988). One group of neurons displays a tonic firing pattern during episodes of locomotion which decrease in frequency or cease with the cessation of locomotion. These neurons have been termed 'on-cells'. A second population of neurons, termed 'off-cells', also displays a tonic firing pattern, however, their frequency of firing decreases as the locomotion frequency increases and conversely their firing rate increases prior to the cessation of locomotion. These two groups of neurons are primarily located within the PPN and are likely to represent subtypes of previously defined type II neurons ('non-bursting' neurons) within PPN. The third group of neurons displays a bursting pattern of firing (termed type I 'bursting' neurons) during locomotion and are located in a more widespread manner, consistent with PPNd glutamatergic neurons.

Experiments on anaesthetized rats showed that about $75 \%$ of PPN neurons showed a regular pattern and $25 \%$ a bursting pattern of activity (Ogura et al 1997). 'On/Off' cells have been proposed to modulate the duration of the stepping episode, while 'bursting' neurons were thought to be involved in modulating frequency (and possibly initiation) of stepping (GarciaRill\&Skinner 1991). Therefore, 'bursting' glutamatergic PPNd neurons, which are primarily innervated by GABAergic GPm and $\mathrm{SNr}$ neurons, and provide the main outputs to the spinal cord, may be important for the initiation of 
programmed movements such as gait. In contrast, 'non-bursting' possibly cholinergic PPNc neurons, which receive most of the sensory feedback information from the spinal cord and provide the main outputs back into the thalamus and SNc, may be important for the maintenance of controlled movements.

In order to elicit locomotion following stimulation of the cat PPN, or any other brainstem site, continuous mid-frequency stimulation of the order of $20-60 \mathrm{~Hz}$ is required. Hundreds of stimuli delivered for several seconds must be applied before the first step is induced (Garcia-Rill \& Skinner 1991). This effect has been interpreted as one of recruitment of locomotion in which spinal pattern generators are activated by reticulospinal systems triggered by stimulation of the brainstem. In contrast, high frequency $(>100 \mathrm{~Hz})$ stimulation of the cat PPN induces a suppression of muscle tone (Lai\&Siegel 1990). Similarly electrical stimulation in the rat has been reported either to reduce or increase muscle tone dependant on the rate of stimulation (Kelland \& Asdourian 1989, Lai \& Siegel 1990). Stimulation of the PPN with excitatory amino acid agonists or with GABA antagonists also increases motor activity. It may be possible that inhibitory GABAergic inputs from GPm or SNr neurons to the glutamate containing PPN neurons normally serve to inhibit locomotor activity such that attenuation of this inhibition by GABAergic blockade (or an optimal electrical frequency stimulation) can elicit locomotion.

Similar experiments in primates are absent, and in humans we can only glean information from anecdotal case reports. Young found that electrical stimulation in a parkinsonian patient in the region of the PPN produced increased tone in the contralateral leg (Young 1992). Masdeau et al (1994) described an inability of a patient to stand or generate stepping after a hemorrhage into the tegmentum of the posterior midbrain. Although the author emphasized the damage to the PPN as the probable cause, the damage 
extended beyond the PPN boundaries and therefore firm conclusions can not be drawn from this case.

\section{THE PPN AND PARKINSON'S DISEASE}

Neuropathological studies on humans have shown that about $50 \%$ of the large cholinergic neurons of the lateral part of the PPNc degenerate in PD (Hirsch et al 1987, Jellinger 1988, Zweig et al 1989). The extent of neuronal loss within the PPNc reflects the extent of neuronal loss in the SNc (Zweig et al 1989). The extent of non-cholinergic neuronal loss within the PPN in PD in not known. It has been proposed that abnormalities in gait and posture, in addition to rigidity and bradykinesia may, in part, be a result of neuronal loss or suppression of activity within PPN. Analysis of the limited clinical data available suggests a relationship between loss of cholinergic neurons in the PPN and severity of PD symptoms (Zweig et al 1989). Thus, the evolving progression of PD and possibly the change in response to L-dopa as the disease progresses, may reflect increasing involvement of certain nondopaminergic neuronal systems, such as the PPNc cholinergic and PPNd glutamatergic systems.

In PD the inhibitory GABAergic projections from the GPm and $\mathrm{SNr}$ to the PPN are as likely to be overactive as are those to the thalamus from these structures as has previously been shown. Also, many GPm and $\mathrm{SNr}$ neurons projecting to the thalamus send collaterals to the PPN (Harnois\&Fillon 1982). Regional uptake studies of 2-deoxyglucose into the PPN in MPTP treated monkeys show increased synaptic activity in the PPN (Mitchell et al 1989). It is not known however, what net effect this increased synaptic activity has on the overall output of PPN neurons. Recent experiments on 6hydroxydopamine (6-OHDA) lesioned rats have demonstrated decreased activity in PPN neurons in the parkinsonian state (Ogura et al 1997). How the populations of cholinergic and non-cholinergic PPN neurons were affected is 
not reported. Similar studies on humans or the relevant electrophysiological studies have as yet not been performed in animal models. Increased GABAergic inhibition of the PPN locomotor region neurons, by overactive descending pallidal and nigral efferents may, therefore, underlie the akinesia and gait difficulties of parkinsonism. This is the hypothesis underlying the present work. 


\section{CHAPTER TWO}

THERMOCOAGULATIVE LESIONS OF THE

PEDUNCULOPONTINE NUCLEUS IN THE

NORMAL SUB-HUMAN PRIMATE 


\section{THERMOCOAGULATIVE LESIONS OF THE PEDUNCULOPONTINE NUCLEUS IN THE NORMAL SUB-HUMAN PRIMATE}

\section{INTRODUCTION}

As we have discussed, due largely to work carried out in monkeys treated with 1-Methyl-4-phenyl-1,2,3,6-tetrahydropyridine (MPTP), we have over the last ten years seen an exponential rise in our understanding of the neural mechanisms underlying the cardinal symptoms of Parkinson's Disease. Loss of the nigro-striatal dopaminergic projection has been shown to result in overactivity of the medial pallidal (GPm) and substantia nigra pars reticulata (SNr) inhibitory output, which in turn depresses the motor activity of the thalamic and brainstem structures to which they have been shown to project, (Aziz et al 1992), leading to the clinical manifestations of akinesia.

The previous hypothesis that akinesia results from ascending, excessive inhibitory activity from the GPm on to cortical areas via a thalamocortical pathway fails to explain the lack of, or adverse, effects on akinesia following thalamotomy (Laitinen et al 1973). That akinesia is improved by both GPm (Baron et al 1996) or STN lesioning or stimulation (Aziz et al 1992, Limousin et al 1995, Benazzouz et al 1996) would support a role for descending basal ganglia outputs in the generation of akinesia, as thalamic lesioning does not affect the descending outputs of the basal ganglia, whereas pallidotomy and subthalamic nucleotomy both do. A logical conclusion would be that overactivity of descending projections to the pedunculopontine area in the upper brainstem (Jackson et al 1983, Aziz et al 1992), rather than overinhibition of the thalamic motor nuclei, is responsible for the akinesia of 
Parkinson's Disease. In support of this hypothesis the optimal target for the relief of akinesia by pallidotomy is the posteroventral third of GPm, the region which has been demonstrated to project mainly to the PPN (Takada 1995). Also, post mortem studies have revealed that in Parkinson's Disease there is a depletion of neurons in the PPN region (Zweig et al 1989). That the PPN plays a crucial role in the control of proximal limb movement would therefore seem a reasonable supposition; and further elucidation of this role, and the part which it may play in the development of akinetic syndromes, would improve our understanding of such states and our attempts at effective therapy.

In this study therefore, I have examined the effect of creating radiofrequency lesions within the PPN region in the normal macaque monkey, thus generating an akinetic state. Our findings support the hypothesis that the PPN plays an important role in normal locomotion and limb movements.

\section{MATERIALS AND METHODS}

Six elderly, ex-breeding macaque female monkeys were used in this study, their mean age was $16 y e a r s ~ 7$ months (range 14years 6 months - 18years 10 months), and mean weight was $6.4 \mathrm{~kg}$ (range $5.7-7.2 \mathrm{~kg}$ ). They were housed in accordance with the Home Office code of practice for the housing and care of animals used in scientific procedures, with all animals housed in a cage of dimensions suitable for those in the 6-9kg category, i.e. Minimum floor space $14,000 \mathrm{~cm}^{2}$, with a minimum height of $150 \mathrm{~cm}$. A fixed 12 hour light dark cycle (07:30 - 19:30hrs lights on, 19:30 - 07:30hrs lights off) was adhered to throughout, and ad lib food and drink provided at all times, except on the 
morning prior to surgery. Under these circumstances no animals exhibited any signs of environmental stress.

Mounted above the home cage was a Doppler activity meter that covered a conical field encompassing the cage area. The meter's sensitivity was set so as to require whole limb or whole body movements to register a single count, the objective being to record the overall activity of the animal during each 24 hour period. The gain of the activity meter was checked and adjusted for each monkey to account for the variation in physical size between animals. Counts were taken at the same time daily to represent a total count for the preceding 24-hour period. Activity monitoring by this method was performed for a minimum period of 7 days prior to surgery in order to provide a baseline 24hour activity count for each animal (N.B. Pre-operative activity monitoring was only commenced once the necessary gain adjustments had been established for each individual animal).

Video recordings of both provoked and unobserved activity were performed to provide a record of speed and nature of movements, facial expression and responsiveness to changes in the surrounding environment. In some instances periods of unobserved recording extended into the animal's 12 hour dark cycle period. However, analysis of sleep behavior patterns has not been included in our present analysis.

The methods of assessment described were performed in an identical manner both pre and post operatively. 


\section{Surgery}

In large sub-human primates it is not possible to utilize bony landmarks for intracranial target acquisition as is practiced in rodents and cats. This is because primates show a large individual variation in the size of the brain and its internal structures. Therefore the accuracy of averaged co-ordinates taken from standard species specific atlases is poor. In an effort to minimize these inaccuracies target acquisition was performed using contrast ventriculography. The linear distance between the anterior and posterior commisures (the ACPC line) was measured on the ventriculogram to accurately reflect the animal's individual brain size. The ratio of the animal's AC-PC line to that of the standard atlas (Shanta et al 1968) gave the proportion by which all coordinates must be increased or decreased for each individual animal. A second potential source of inaccuracy which must be corrected for in ventriculographic targeting, was the magnification created by divergent $\mathrm{X}$-ray beams set at a distance from the target film. To account for this, included in our targeting apparatus was a side arm which contained two vertical $1 \mathrm{~mm}$ tip steel pins which were set in Perspex such that the gap between the pin tips was exactly $10 \mathrm{~mm}$. On each occasion the side arm was fixed to the operating table so that the 'pin gap' lay just lateral to the external auditory meatus (EAM) of the positioned animal. A 'template' X-ray was performed with the side arm in situ and the distance between the pin tips on X-ray measured on the X-ray (distance $\mathrm{Xmm}$ ), thus the $\mathrm{X}$-ray magnification fraction can be assessed and corrected for on each occasion as, $(\mathrm{X}$-ray magnification $=\mathrm{X} / 10)$.

Prior to surgery the electrode to be used for lesioning, which in all cases was a $1.0 \mathrm{~mm} \times 1.0 \mathrm{~mm}$ exposed tip stimulating/lesioning electrode, was placed in the 
horizontal holder of the microdrive which was fixed to the lateral edge of the operating table. This was a calibrated microdrive by which movements with a resolution of $0.1 \mathrm{~mm}$ in all three planes may be made. This was then positioned with the tip of the electrode tip-to-tip with the posterior pin of our previously fixed and X-rayed side arm. In this position the co-ordinates of the microdrive in the AP and DV planes ( $x$ and $z$ ) are recorded for later target calculations.

The animals were fasted on the night prior to surgery. On the day of surgery all animals were initially sedated with a single dose of intramuscular ketamine (10 $\mathrm{mg} / \mathrm{kg}$ body weight). Thereafter anesthesia was maintained by means of a continuous intravenous infusion of althesin titrated according to the animals' weight $(6 \mathrm{mg} / \mathrm{kg} / \mathrm{hr})$, and administered via an infusion pump. When anaesthetized the animals head was shaved prior to being placed with the skull immobilized anteriorly by means of a mouth piece placed under the maxilla and hard palate inferiorly, and superiorly a pair of eye bars which fixed to the inferior orbital ridge on either side of the animals head. With this fixation the orbito-meatal line was adjusted until it lay parallel to the operating table surface. Having positioned the animal thus, a check X-ray was taken to ensure that the saggittal plane of the animal lay perpendicular to the X-ray beam. All surgery was performed under aseptic conditions, and all animals received intramuscular antibiotic prophylaxis (Synulox $0.06 \mathrm{ml} / \mathrm{kg}$ ) on the day prior to surgery, on induction of anesthesia and for 2 days post operatively. The skin was prepared with an alcohol/iodine solution and a linear incision sited over the saggittal suture. In order to perform a ventriculogram a burr hole was made at $20 \mathrm{~mm}$ anterior to the external auditory meatus (EAM) which exposed the 
superior saggittal sinus in the midline, and extended $2 \mathrm{~mm}$ lateral to this plane. At this time the laterality of the midline, as read on the microdrive was assessed by siting the electrode tip, within the sidearm holder of the microdrive, over the sinus and reading the lateral co-ordinate of the microdrive $(y)$. The dura at $2 \mathrm{~mm}$ lateral to this was incised, and through this, a 23 gauge sterile needle was passed vertically into the anterior horn of the lateral ventricle, as confirmed by the aspiration of cerebrospinal fluid (CSF), usually at a depth of $15-20 \mathrm{~mm}$ dependant on the individual animal's size. Attached to the needle, and held in the microdrive arm of the frame, was a $1 \mathrm{ml}$ syringe containing contrast medium (Omnipaque 300, Nycomed UK). On free aspiration of CSF $0.5-1.0 \mathrm{ml}$ of Omnipaque was injected into the ventricular system and a lateral X-ray taken (Fig. 2.1a,b).

Following contrast ventriculography and proportional correction for size with reference to a standard atlas (Shanta 1968) and magnification, a target was calculated in the PPN, $2.0 \mathrm{~mm}$ lateral to the midline and $6.0 \mathrm{~mm}$ below the posterior comissure. The method used for correction and target calculation is illustrated in Table 2.1.

In order to site our lesion a second burr hole was made at the point on the skull which the electrode lay above when the AP and Lateral co-ordinates for the target were set on the side arm, thus allowing access at the appropriate point of the skull to set the DV co-ordinate for our calculated target. In the case of animals undergoing simultaneous bilateral lesions a single burr crossing the midline was made at this point. The dura beneath our burr hole was incised in order to prevent the possibility of any deviation of our electrode tip, and then the electrode advanced through the incised area such that the 
required vertical setting for targeting be achieved with the AP and Lateral coordinates for target having previously been set. At this point a further lateral radiograph was performed (Fig. 2.2). At this point, with the electrode sited in the previously targeted PPN as confirmed on our repeat X-ray, a stimulating current of frequency $2 \mathrm{~Hz}$ and amplitude 100milliseconds was applied a voltage no greater than $1 \mathrm{~V}$. A consistent finding was twitching of the levator palpebrae muscle on the stimulated side. Next a lesion was made by heating the exposed tip to $80^{\circ} \mathrm{C}$ for 60 seconds using a Radionics RFG lesion generator as had been used for the prior stimulation through the same electrode. The electrode was allowed to cool to a temperature below $40^{\circ} \mathrm{C}$ prior to its' removal in order to prevent thermal damage along the electrode tract.

In three animals a unilateral lesion was first made with a second contralateral lesion being made one week later. In a further three animals bilateral lesions were made during a single operation. An intramuscular dose of buprenorphine $(0.06 \mathrm{ml} / \mathrm{kg}$ body weight) was administered at the end of each procedure to allow for postoperative analgesia. All animals were maintained on an intravenous hydration regime $(40 \mathrm{ml} / \mathrm{kg} / 24 \mathrm{hrs}$ Normal Saline) until they were able to safely maintain adequate oral hydration. The surgical incisions were closed with interrupted sutures of 3/0 coated Vicryl and the animals returned to the home cage where they were cared for on Vetbed and under an ultraviolet heat lamp for the initial 12-24 hours post operatively.

Following recovery from anesthesia all animals were monitored in an identical fashion to that which had been applied pre-operatively (daily activity counts and video recording) for a 7 day period. After this period the animals were sacrificed by means of an intravenous overdose of sodium pentobarbital 
(60 mg/kg body weight), and perfused transcardially with physiological saline followed by $10 \%$ formalin in a $0.1 \mathrm{M}$ phosphate buffer. The heads were then remounted in the stereotactic frame and the region of interest removed en bloc and placed in the same buffered formalin solution containing $30 \%$ sucrose. After a period of 7 days fixation in the sucrose/formalin solution, the tissues were cut serially into 50 -micrometer sections on a freezing microtome. Alternate sections were then mounted and stained with Cresyl Violet (CV) to assess lesion placement and size, some sections were also stained with Luxol Fast Blue (LFB) to assess the integrity of fibre tracts (Fig. 2.3a,b,c,d,e,f). 
Figure 2.1a. Ventriculogram in Macaque Showing External Pins Used for Target Calculation and Assessment of Magnification.

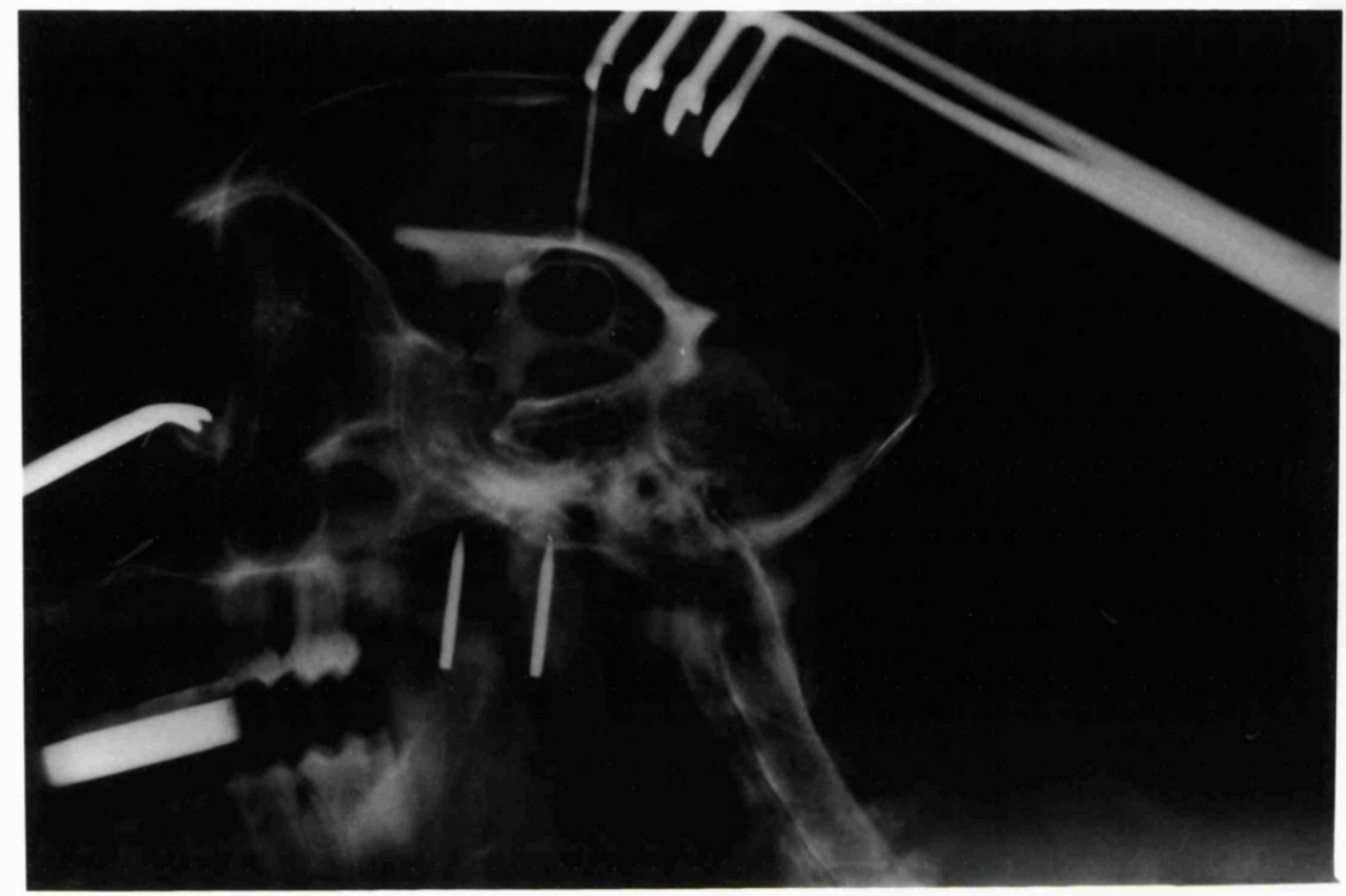

Figure 2.1b. Tracing of Above Ventriculogram marking AC, PC and the Calculated Target. (3V - third ventricle, LV - lateral ventricle).

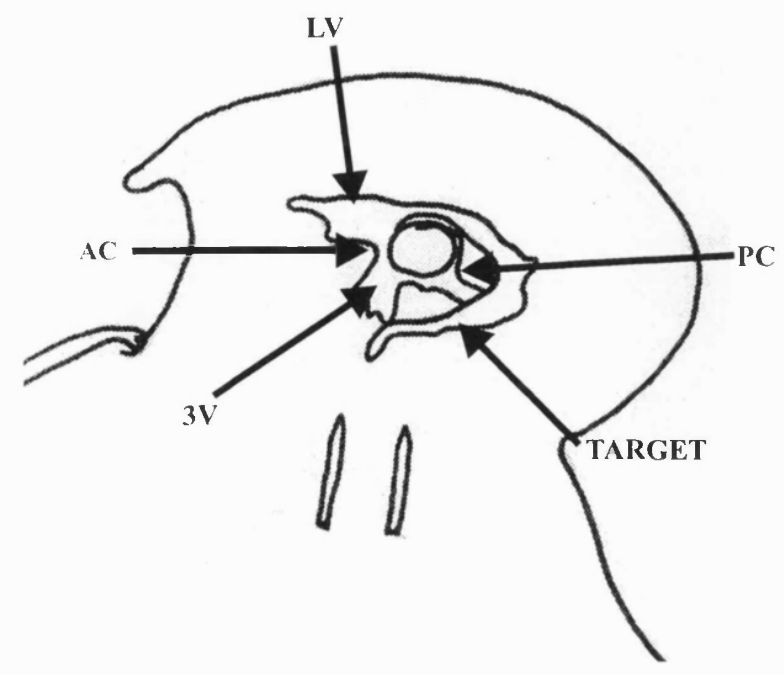


64

Figure 2.2. Lateral Radiograph in Macaque Showing Electrode Inserted to Target Co-Ordinates. 
TABLE 2.1. Correction of Data and Calculation of PPN

Co-ordinates In Thermocoagulative Lesioning.

ELECTRODE ZERO : $\quad \mathrm{AP}=x$

(i.e.tip-to-tip with posterior pin) Vert $=z$

MIDLINE AT SAGGITTAL SINUS: Lat $=\mathrm{y}$

MAGNIFICATION : Actual distance between pin tips $=10 \mathrm{~mm}$

$\mathrm{X}$-ray distance between pin tips $=\mathrm{Xmm}$

Magnification factor $=\mathrm{X} / 10=\mathrm{M}$

X-RAY AC-PC DISTANCE [cm] :A

ACTUAL AC-PC DISTANCE $[\mathbf{c m}] \quad: \mathrm{A} / \mathrm{M}=\mathrm{E}$

ENLARGEMENT FACTOR OFF ATLAS: E $1=\mathrm{E}$ (as atlas AC-PC $=1 \mathrm{~cm}$ )

TARGET FROM ATLAS $\quad: 6 \mathrm{~mm}$ below PC

$2 \mathrm{~mm}$ lateral to midline

TARGET CORRECTED OFF ATLAS : $6 \mathrm{x}$ E below PC

TARGET ON X-RAY

$2 \times$ E lateral to midline

: $(6 \times \mathrm{E}) \mathrm{x}$ M below PC

$(2 \times$ E) lateral to midline

TARGET RELATIVE TO POSTERIOR PIN ON X-RAY:

$$
\begin{aligned}
\mathrm{AP} & =\chi \mathrm{ant} / \mathrm{pos} t / \text { to } \mathrm{pin} \\
\mathrm{Vert} & =\delta \text { sup/inf to } \mathrm{pin}
\end{aligned}
$$

ACTUAL TARGET RELATIVE TO POSTERIOR PIN:

$\mathrm{AP}=\chi / \mathrm{Mmm}=$ Amm ANTERIOR/POSTERIOR to pin

Vert $=\delta / M=$ Dmm SUPERIOR/INFERIOR to zero

\section{RIGHT CO-ORDINATES LEFT CO-ORDINATES}

AP

$\mathbf{x}+l-\mathbf{A}=\mathbf{A P}$

Lat $\quad \mathbf{y}+(2 \times E)=$ RLat

$y-(2 \times E)=$ LLat

Vert

$z+1-\mathbf{D}=\mathbf{D V}$

In AP plane ANTERIOR $=+\mathrm{A}$

In VERT plane SUPERIOR $=+\mathrm{D}$

POSTERIOR $=-A$

INFERIOR $=-\mathrm{D}$ 
Figure 2.3 a-f. Histological Sections in Monkeys 1 to 6 Showing Site and Size of Radiofrequency Lesions.

(a,b,e - stained with LFB c,d,f - stained with CV)

a.) Monkey 1

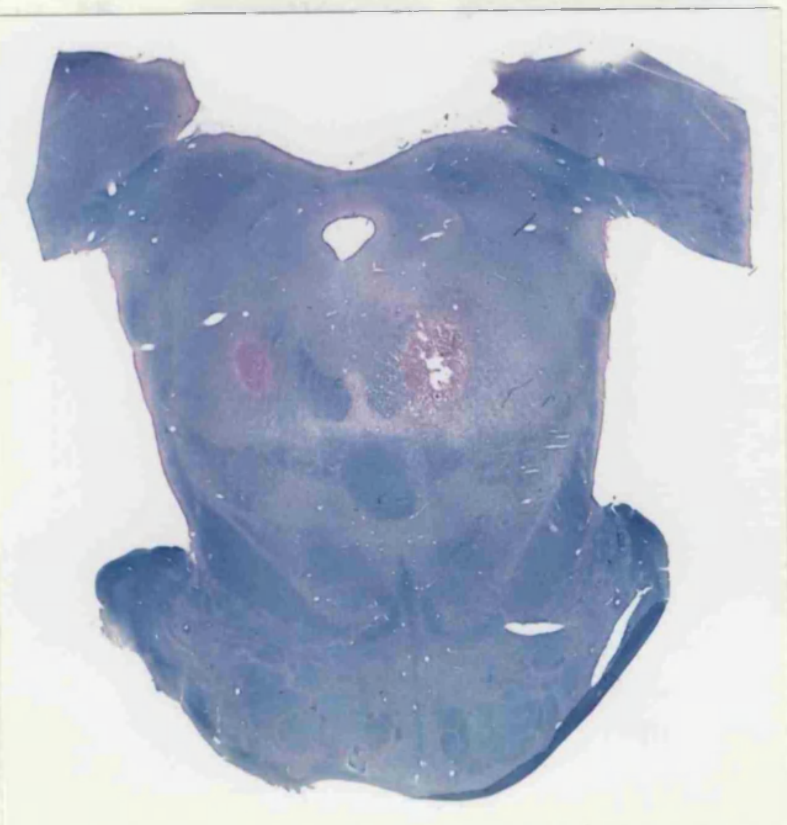

b.) Monkey 2

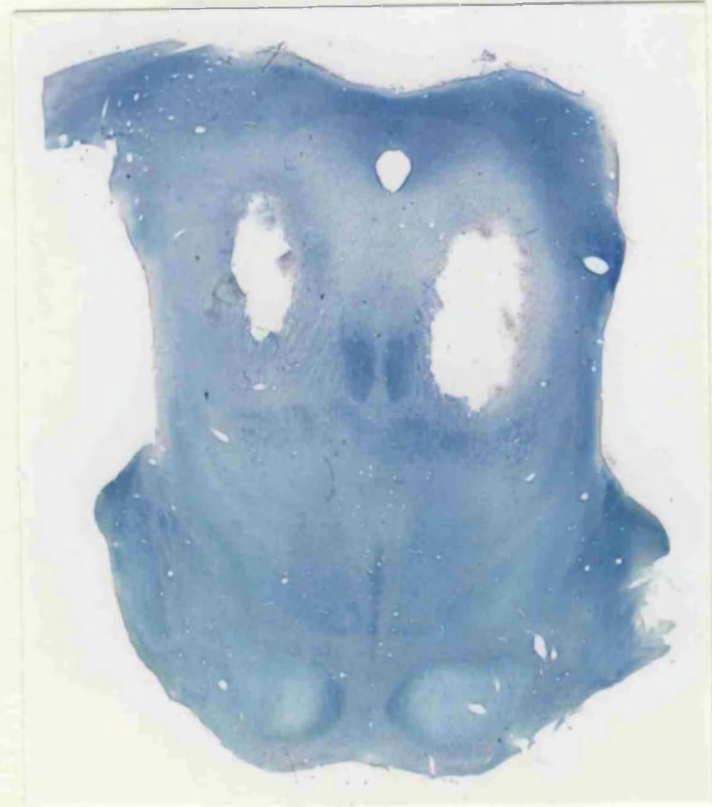


c.) Monkey 3

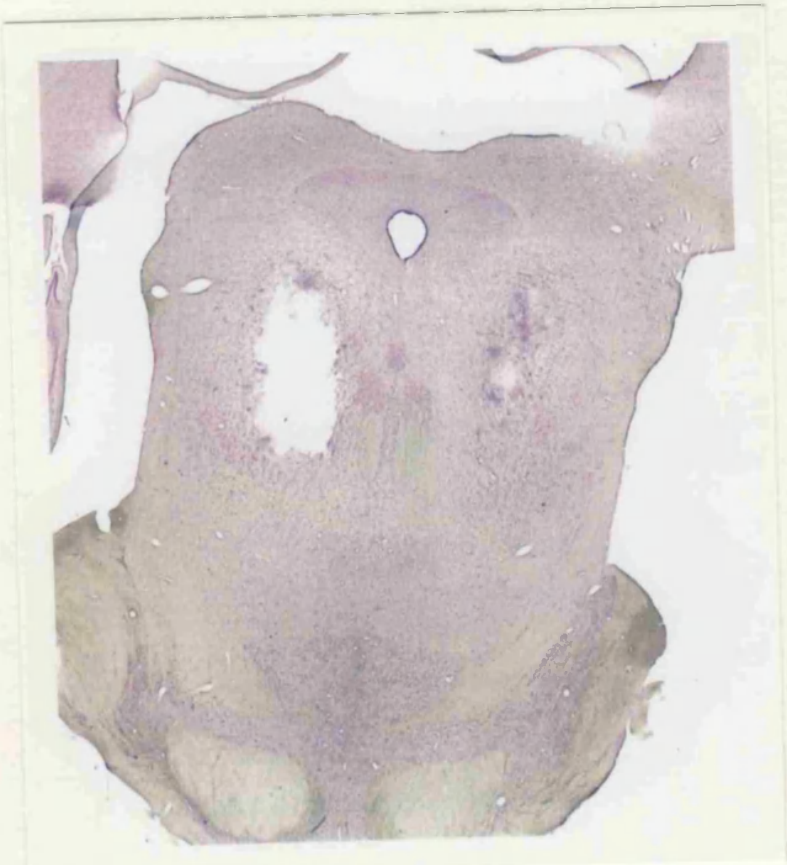

d.) Monkey 4

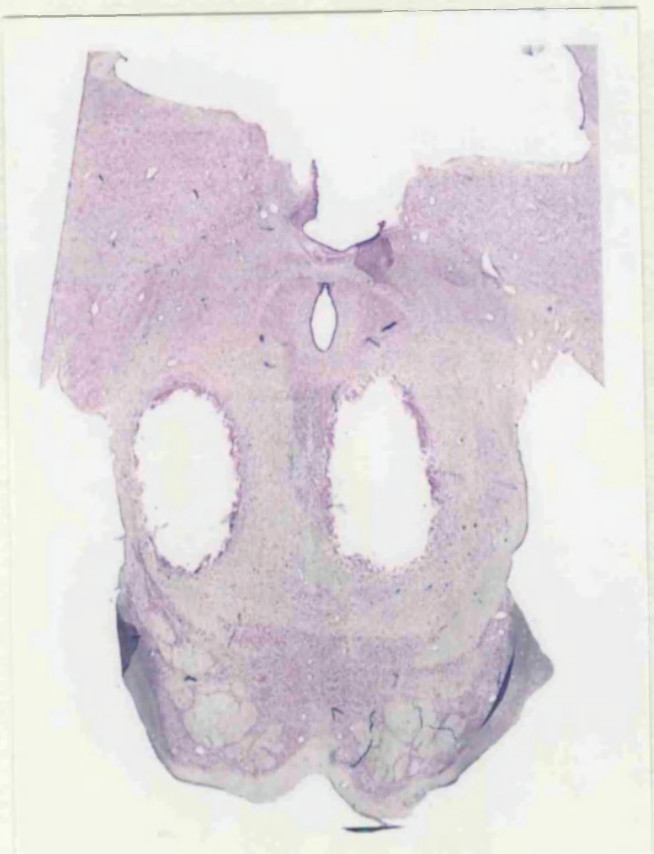


e.)Monkey 5

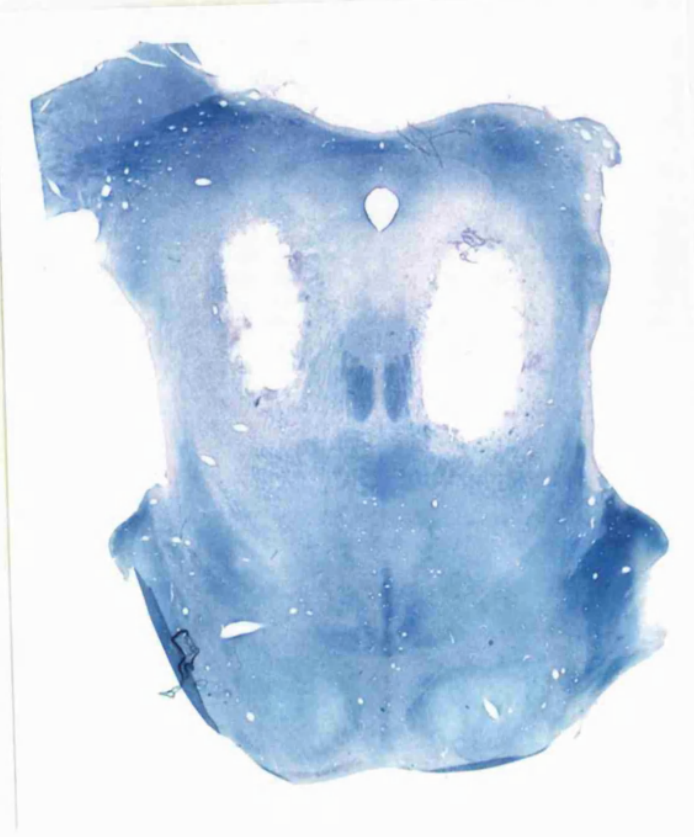

f.) Monkey 6

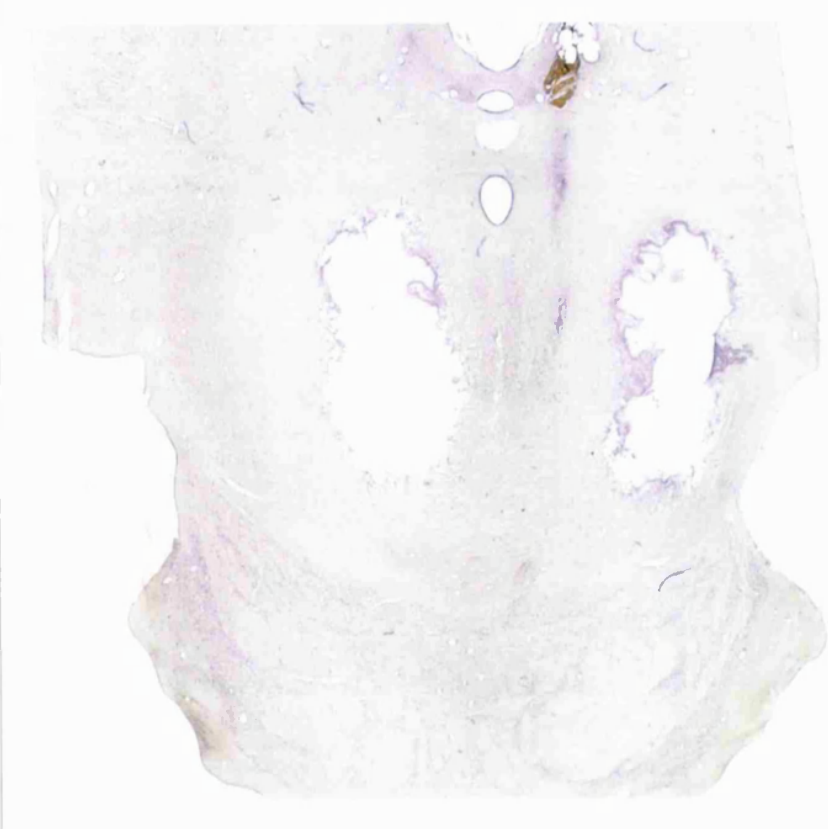




\section{RESULTS}

Due to the relatively small number of animals we were able to study, results

for individual animals will be given first, then the combined results discussed.

\section{Monkey 1}

Demographics : Female adult Rhesus

Age 17 years 4 months

\section{Weight $6.2 \mathrm{~kg}$}

Pre-operative Assessment:

a.) $24 \mathrm{hr}$ activity counts $-1011 / 1364$ / $1239 / 1114 / 1097 / 1337 / 1357$

Mean 24 hour activity count of $\mathbf{1 2 1 7}$ counts/day.

c.) Video recording - no observed atypical behavior.

Normal range of facial expression.

Surgical Procedures:

a.)Day 0 - Left PPN thermocoagulative lesion performed

As described in materials and methods (Appendix 1).

b.)Day 8 - Right PPN thermocoagulative lesion performed

As described in materials and methods (Appendix 1).

Post Operative Assessment:

a.) $24 \mathrm{hr}$ activity counts $-648 / 695 / 711 / 720 / 837 / 1248 / 1625$

Average 24hour activity count of $\mathbf{9 2 6}$ counts/day

post unilateral lesion.

$708 / 720 / 718 / 726 / 644 / 704 / 754$

Average 24 hour activity count of $\mathbf{7 1 0}$ counts/day

post contralateral lesion.

c.)Video Recording - mild bradykinesia, hypokinesia and poverty of

facial expression noted post bilateral lesions. 


\section{Monkey 2}

Demographics : Female adult Rhesus

Age 14 years 6 months

Weight $5.8 \mathrm{~kg}$

Pre-operative Assessment:

a.) 24 hr activity counts -1109 / 1472 / $1366 / 1298 / 1449$ / $1401 / 1551$

Average 24 hour activity count of $\mathbf{1 3 7 8}$ counts/day.

c.) Video recording - no observed atypical behavior.

Normal range of facial expression.

Surgical Procedures:

a.)Day 0 - Left PPN thermocoagulative lesion performed

As described in materials and methods (Appendix 1).

b.)Day 8 - Right PPN thermocoagulative lesion performed

As described in materials and methods (Appendix 1).

Post Operative Assessment:

a.) 24 hr activity counts $-583 / 825 / 1126 / 1490 / 1392 / 1380 / 1445$

Average 24hour activity count of $\mathbf{1 1 7 6}$ counts/day post unilateral lesion.

$211 / 163 / 218 / 326 / 437 / 355 / 441$

Average 24 hour activity count of $\mathbf{3 0 7}$ counts/day post contralateral lesion.

c.)Video Recording - marked bradykinesia, hypokinesia and poverty of facial expression noted post bilateral lesions. 


\section{Monkey 3}

Demographics : Female adult Rhesus

Age 16 years 3 months

Weight $6.7 \mathrm{~kg}$

Pre-operative Assessment:

a.)24hr activity counts - 3234 / $2967 / 2842$ / $3179 / 2715 / 3011 / 2667$

Average 24 hour activity count of 2945 counts/day.

c.) Video recording - no observed atypical behavior.

Normal range of facial expression.

Surgical Procedures:

a.)Day 0 - Left PPN thermocoagulative lesion performed

As described in materials and methods (Appendix 1).

b.)Day 8 - Right PPN thermocoagulative lesion performed

As described in materials and methods (Appendix 1).

Post Operative Assessment:

a.)24hr activity counts $-835 / 1542 / 2611 / 2894 / 2729 / 2554 / 2704$

Average 24 hour activity count of 2267 counts/day post unilateral lesion.

$638 / 437 / 325 / 339 / 256 / 281 / 254$

Average 24 hour activity count of 361 counts/day post contralateral lesion.

c.)Video Recording - marked bradykinesia, hypokinesia and poverty of facial expression noted post bilateral lesions. 


\section{Monkey 4}

Demographics : Female adult Rhesus

Age 16 years 9 months

Weight $7.2 \mathrm{~kg}$

Pre-operative Assessment:

a.) $24 \mathrm{hr}$ activity counts -2032 / $2211 / 1947$ / 2269 / 2187 / $2310 / 2164$

Average 24hour activity count of 2160 counts/day.

c.) Video recording - no observed atypical behavior.

Normal range of facial expression.

Surgical Procedures:

a.)Day 0 - Bilateral PPN thermocoagulative lesions performed As described in materials and methods (Appendix 1).

Post Operative Assessment:

a.) $24 \mathrm{hr}$ activity counts $-121 / 150 / 256 / 420 / 445 / 455 / 420$

Average 24hour activity count of 324 counts/day post bilateral lesions.

c.)Video Recording - marked bradykinesia, hypokinesia and poverty of facial expression noted post bilateral lesions. 


\section{Monkey 5}

Demographics : Female adult Rhesus

Age 14 years 11 months

Weight $5.7 \mathrm{~kg}$

Pre-operative Assessment:

a.)24hr activity counts $-985 / 1111 / 1230 / 965 / 1027 / 1352 / 1044$

Average 24hour activity count of $\mathbf{1 1 0 2}$ counts/day.

c.) Video recording - no observed atypical behavior.

Normal range of facial expression.

Surgical Procedures:

a.)Day 0 - Bilateral PPN thermocoagulative lesions performed As described in materials and methods (Appendix 1).

Post Operative Assessment:

a.)24hr activity counts $-451 / 320 / 287 / 278 / 301 / 324 / 299$

Average 24 hour activity count of 323 counts/day post bilateral lesions.

c.)Video Recording - marked bradykinesia, hypokinesia and poverty of facial expression noted post bilateral lesions. 


\section{Monkev 6}

Demographics : Female adult Rhesus

Age 18 years 10 months

Weight $6.8 \mathrm{~kg}$

Pre-operative Assessment:

a.) $24 \mathrm{hr}$ activity counts $-1189 / 1372 / 1308 / 1297 / 1246 / 1019 / 1207$

Average 24hour activity count of $\mathbf{1 2 3 4}$ counts/day.

c.) Video recording - no observed atypical behavior.

Normal range of facial expression.

Surgical Procedures:

a.)Day 0 - Bilateral PPN thermocoagulative lesions performed

As described in materials and methods (Appendix 1).

Post Operative Assessment:

a.)24hr activity counts $-508 / 435 / 501 / 455 / 402 / 450 / 461$

Average 24 hour activity count of $\mathbf{4 5 9}$ counts/day

post bilateral lesions.

c.)Video Recording - marked bradykinesia, hypokinesia and poverty of facial expression noted post bilateral lesions. 


\section{SUMMARY OF RESULTS}

Within an hour monkeys recover fully from althesin anesthesia. After the lesions they made many fewer spontaneous movements and those that they did make were clearly very much slower, as if they were 'moving through treacle'. Interestingly their movements were still well coordinated, and showed no signs of cerebellar ataxia or intention tremor even though they were so much slower. But their automatic movements, such as reflex withdrawal to noxious stimuli, were brisk and seemed unaffected by the lesions.

Normal rhesus monkeys make a large variety of facial movements including frequent 'threat' expressions - baring the teeth, abduction of the corners of the mouth and pushing the head forward. Immediately after the lesions however, the animals' faces were strikingly immobile with fixed, impassive, expressions and they made no threat attempts at all. These features were very reminiscent of Parkinson's patients.

The first day after unilateral lesions the quantity and speed of the monkeys' movements reduced to approximately one third of their preoperative level (Table 2.2), and their facial expressions became fixed. The reduction in their activity was highly statistically significant $(t=3.6, p<0.01)$. However, they recovered to $55 \%$ of control values on the second day and returned to normal over the next week (Figs. 2.4 and 2.5).

After the second lesion in the first three monkeys or bilateral lesions in the subsequent three monkeys, the poverty and slowing of the animals movements showed little improvement in the week following surgery. On the first post operative day activity counts had dropped by $74 \%$ (Table2.3, $[\mathrm{t}=5.5$ $, \mathrm{p}<0.01]$ ), and on the seventh day they were still reduced by over $70 \%$ (Figs 2.6 and 2.7).Also the monkeys' lack of facial expression persisted, a fact confirmed on reviewing the videotapes. 
Histologicaly, all lesions included all or part of the PPN region on both sides. It is not possible to distinguish between the cholinergic and glutamatergic PPN on these sections, and both may be assumed to be involved. The precise site and size of lesions varied, as did the degree to which the surrounding mesencephalic locomotor region was involved in the lesion (Figs. 2a-f). Staining with Luxol Fast Blue (LFB) demonstrated that the lesions left most of the Red nucleus and the fibers of the superior cerebellar peduncle intact in all cases (Figs. 2.3a,b,e).

Monkeys 3 showed the most rapid recovery following a unilateral lesion. Histology in this animal (Fig 2.3c) shows that the initial left sided lesion was indeed small though placed in the correct region. The best recovery following bilateral lesions was seen in Monkey 1, where histology shows both lesions to be smaller than those in the other animals who demonstrated more marked post operative effects (Fig 2.3a).

Clearly our lesions greatly slowed the animals' movements and reduced their spontaneous activity. The animals recovered in only a few days after unilateral lesions however. This was probably because the ascending and descending projections from each PPN to the basal ganglia and pontine and medullary reticulo-spinal systems are bilateral (Jackson et al 1983) so the intact side could take over. But when the surviving PPN was ablated or both were destroyed at the same time the animals' activity did not recover. These reductions in the monkey's movements therefore support our hypothesis that Parkinsonian bradykinesia results from overinhibition of the PPN by an overactive medial pallidum.

The large lesions which we made probably included not only the cholinergic pedunculopontine nucleus but also adjacent areas of the mesencephalic locomotor region. But it is unlikely that they had a significant effect on cerebellar outflow because staining with Luxol Fast Blue which accentuates fibres, confirmed that the majority of the axons in the the superior 
cerebellar peduncle remained intact. Moreover after the lesions it was remarkable how well coordinated and tremor free the animals' movements remained, despite their rarity and slowness.

Another possibility is that the animals' inactivity was non-specific and nothing to do with the descending pallidal projections. Our lesions might have affected the general reticular activating system. But if they had destroyed a substantial proportion of that system we might have expected the animals to have slept, and not to have moved at all, rather than simply moving much more slowly and less often. The doppler activity meter was sensitive both to the speed of the animals' movements and to their overall level of activity, hence the reduction in counts confirmed our video observation that the animal's moved more slowly after the lesions.

It might also be argued that the lesions affected the animals' motivation to move rather than their ability to do so. Where motivation can be said to end and execution to begin is largely a question of semantics. However if our lesions had eliminated the animals' motivation altogether, again we would have expected them not to move at all. Instead their spontaneous movements were slowed; but their reflex movements were relatively intact. Therefore, it seems likely that the lesions had the specific effects of reducing the number and slowing down the performance of their voluntary movements, without affecting their general arousal or motivation; in short rendering them akinetic as in Parkinson's disease. 
Table 2.2. 24 Hour Activity Counts, and their Averages, in Monkeys 1-3 Following Unilateral Thermocoagulative PPN Lesions.

\begin{tabular}{|c|c|c|c|c|c|c|c|c|}
\hline & $\begin{array}{l}\text { Average } \\
\text { Pre Op }\end{array}$ & $\begin{array}{l}\text { Post Op } \\
\text { Day } 1\end{array}$ & $\begin{array}{l}\text { Post Op } \\
\text { Day } 2 \\
\end{array}$ & $\begin{array}{l}\text { Post Op } \\
\text { Day } 3\end{array}$ & $\begin{array}{l}\text { Post Op } \\
\text { Day } 4\end{array}$ & $\begin{array}{l}\text { Post Op } \\
\text { Day } 5 \\
\end{array}$ & $\begin{array}{l}\text { Post Op } \\
\text { Day } 6\end{array}$ & $\begin{array}{l}\text { Post Op } \\
\text { Day } 7\end{array}$ \\
\hline $\begin{array}{l}\text { Monkey } \\
1\end{array}$ & 1217 & 648 & 695 & 711 & 720 & 837 & 1248 & 1625 \\
\hline $\begin{array}{l}\text { Monkey } \\
2\end{array}$ & 1378 & 583 & 825 & 1116 & 1490 & 1392 & 1380 & 1445 \\
\hline $\begin{array}{l}\text { Monkey } \\
3\end{array}$ & 2945 & 835 & 1542 & 2611 & 2894 & 2729 & 2554 & 2704 \\
\hline $\begin{array}{l}\text { Daily } \\
\text { Average }\end{array}$ & 1847 & $689 * *$ & $1020 *$ & 1479 & 1701 & 1653 & 1727 & 1925 \\
\hline $\begin{array}{l}\text { Standar } \\
\text { d Error }\end{array}$ & 551 & 76 & 263 & 578 & 636 & 562 & 415 & 393 \\
\hline
\end{tabular}


Table 2.3. 24 Hour Activity Counts, and their Averages, in Monkeys 1-3 following a Contralateral lesion and in Monkeys 4-6 Following Bilateral Thermocoagulative Lesions .

\begin{tabular}{|c|c|c|c|c|c|c|c|c|}
\hline & $\begin{array}{l}\text { Average } \\
\text { Pre Op }\end{array}$ & $\begin{array}{l}\text { Post Op } \\
\text { Day } 1 \\
\end{array}$ & $\begin{array}{l}\text { Post Op } \\
\text { Day } 2\end{array}$ & $\begin{array}{l}\text { Post Op } \\
\text { Day } 3 \\
\end{array}$ & $\begin{array}{l}\text { Post Op } \\
\text { Day } 4\end{array}$ & $\begin{array}{l}\text { Post Op } \\
\text { Day } 5\end{array}$ & $\begin{array}{l}\text { Post Op } \\
\text { Day } 6\end{array}$ & $\begin{array}{l}\text { Post Op } \\
\text { Day } 7\end{array}$ \\
\hline $\begin{array}{l}\text { Monkey } \\
1\end{array}$ & 1515 & 708 & 720 & 718 & 726 & 644 & 704 & 754 \\
\hline $\begin{array}{l}\text { Monkey } \\
2\end{array}$ & 1609 & 211 & 163 & 218 & 326 & 437 & 355 & 441 \\
\hline $\begin{array}{l}\text { Monkey } \\
3\end{array}$ & 2825 & 638 & 437 & 325 & 339 & 256 & 281 & 254 \\
\hline $\begin{array}{l}\text { Monkey } \\
4\end{array}$ & 2160 & 121 & 150 & 256 & 420 & 445 & 455 & 420 \\
\hline $\begin{array}{l}\text { Monkey } \\
5\end{array}$ & 1102 & 451 & 320 & 287 & 278 & 301 & 324 & 299 \\
\hline $\begin{array}{l}\text { Monkey } \\
6\end{array}$ & 1234 & 508 & 435 & 501 & 455 & 402 & 450 & 461 \\
\hline $\begin{array}{l}\text { Daily } \\
\text { Average }\end{array}$ & 1724 & $440 * *$ & $371^{* *}$ & $384 * *$ & $424 * *$ & $414^{* *}$ & $428 * *$ & $438 * *$ \\
\hline $\begin{array}{l}\text { Standar } \\
\text { d Error }\end{array}$ & 270 & 95 & 87 & 78 & 66 & 56 & 62 & 72 \\
\hline
\end{tabular}

** $\mathrm{t}>4.0 ; \mathrm{p}<0.01$ comparing preoperative activity counts with those after lesions.

$* t>2.0 ; \mathrm{p}<0.05$ comparing preoperative activity counts with those on $2^{\text {nd }}$ day after first lesion. 
Figure 2.4. 24 Hour Activity Counts in Monkeys 1 - 3 Foliowing Unilateral Thermocoaguiative Lesions of the PPN.

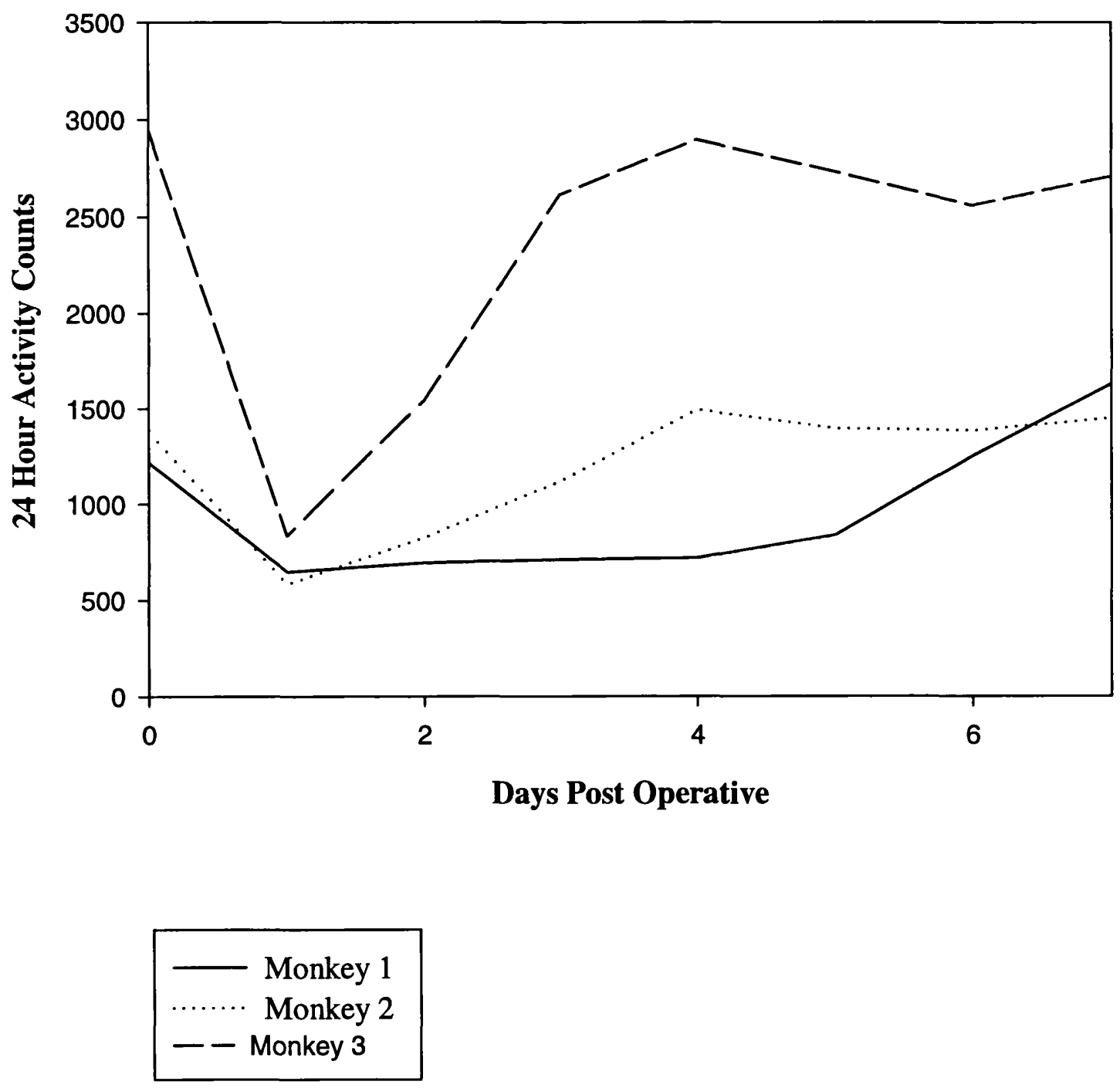


Figure 2.5. Averaged 24 Hour Activity Counts in Monkeys 1 - 6 Following Unilateral Thermocoagulative Lesions of the PPN.

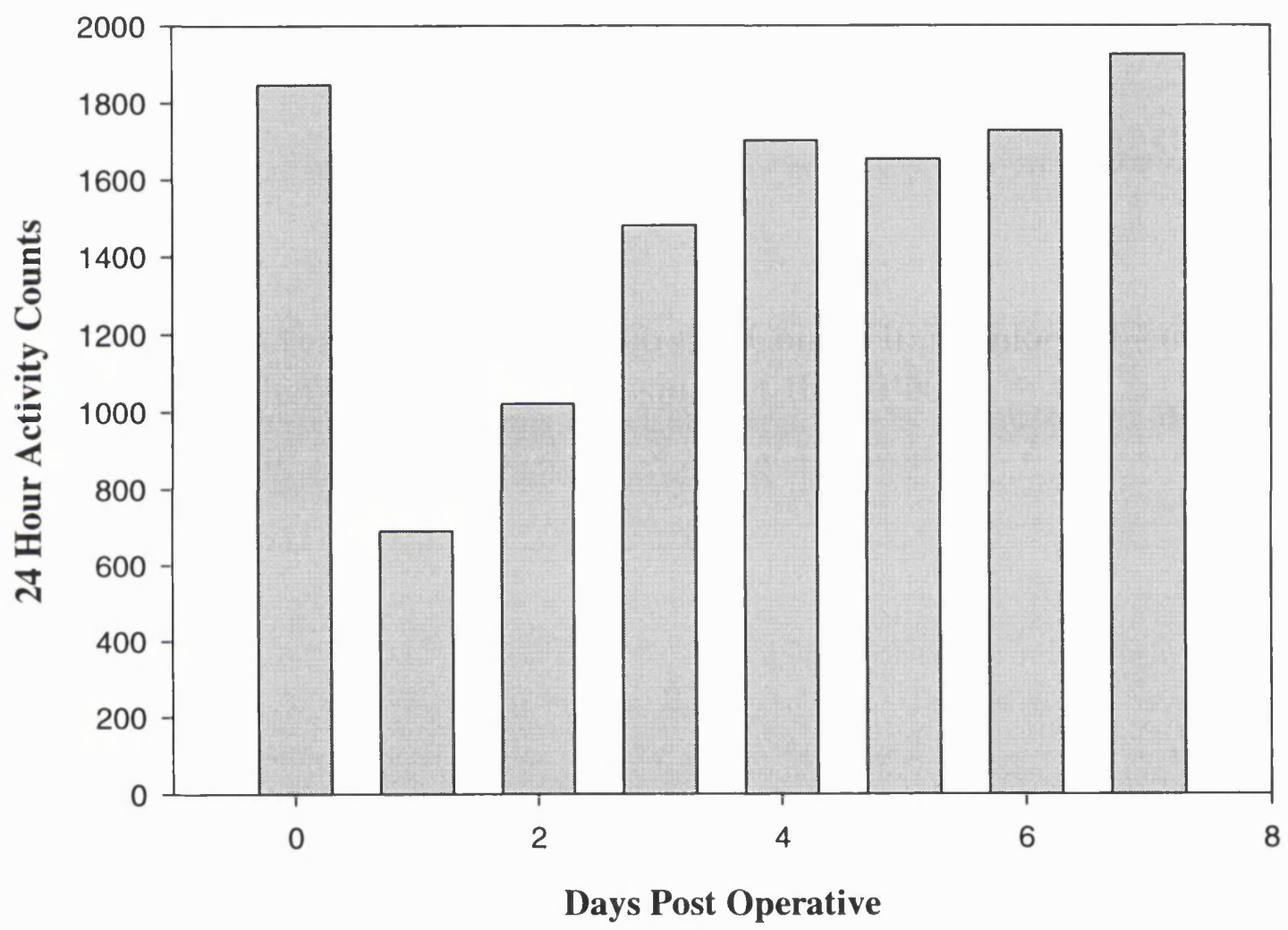


Figure 2.6. Individual 24Hour Activity Counts in Monkeys 1 - 6 Following Bilateral Thermocoagulative Lesions of the PPN.

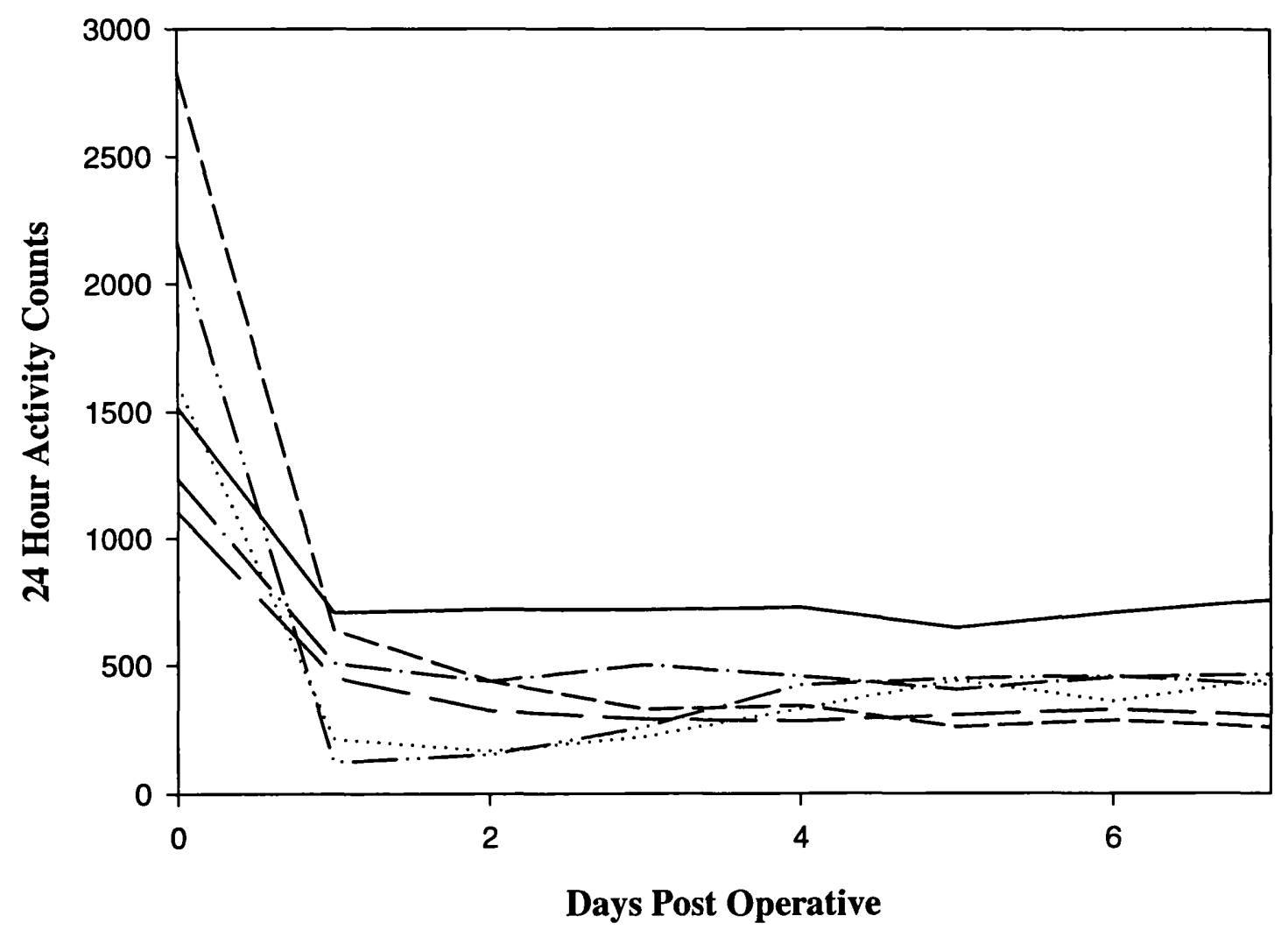

Monkey 1

Monkey 2

- - Monkey 3

-... Monkey 4

- Monkey 5

- - Monkey 6 
Figure 2.7. Averaged 24 Hour Activity Counts in Monkeys 1 - 6 Following Bilateral Thermocoagulative Lesions of the PPN.

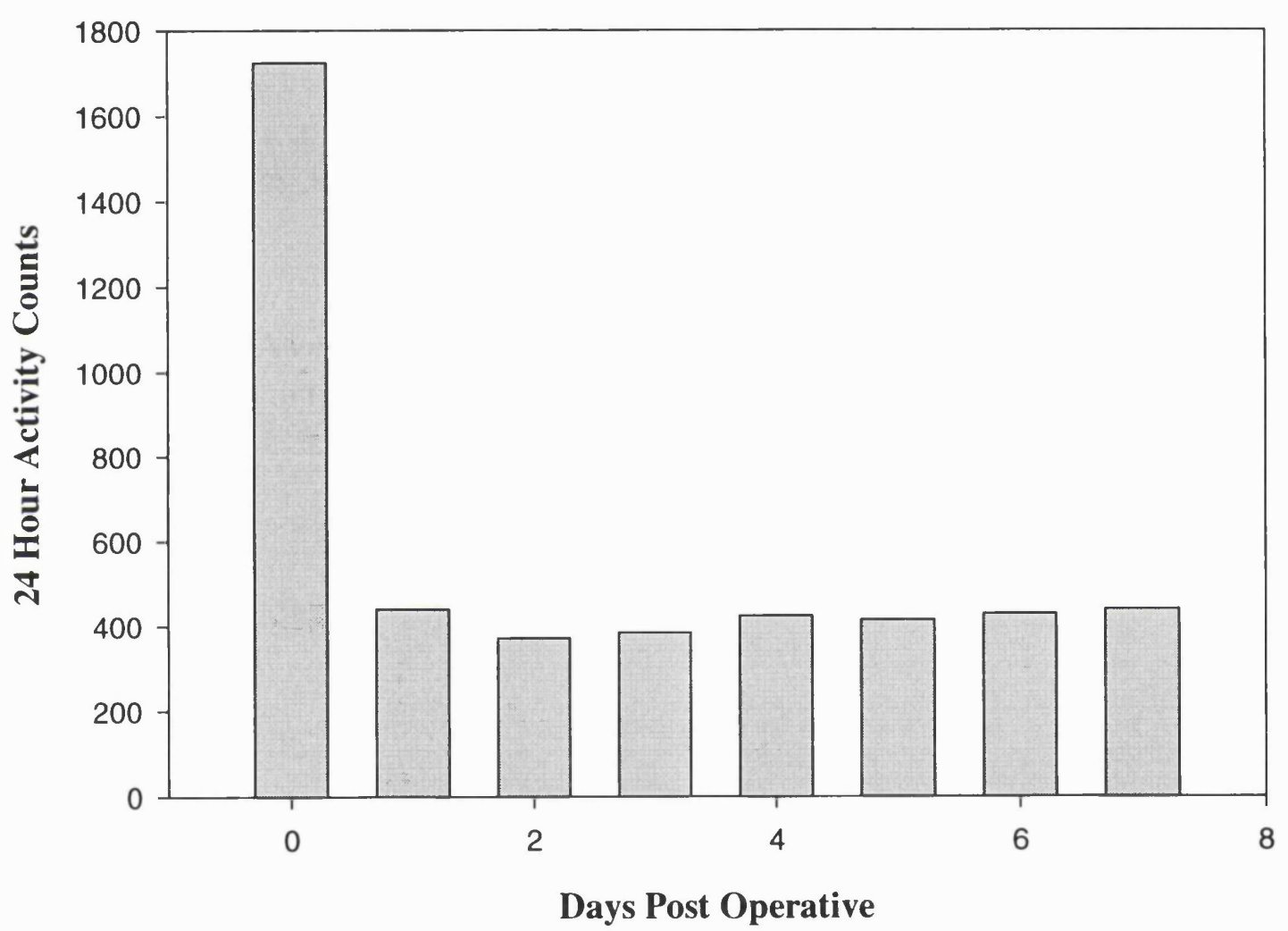




\section{CHAPTER THREE}

THE DESIGNING OF A STEREOTACTIC FRAME FOR USE IN THE SUB-HUMAN PRIMATE 


\section{THE DESIGNING OF A STEREOTACTIC FRAME FOR USE IN THE SUB-HUMAN PRIMATE}

\section{THE NEED FOR A NEW FRAME}

Having shown that large radiofrequency lesions of the PPN region cause akinesia we wished to confirm that more limited excitotoxic lesions would have the same effect. We therefore wished to further improve the accuracy of our targeting by using a framed system of stereotaxy. The lesions which we had created using the radiofrequency method previously described undoubtedly included the PPN region, but were larger than the lesions which we could anticipate making using an injected cytotoxic agent. Therefore, any means by which we may be able to further ensure accurate targeting of a structure having a maximum diameter of 2-3mm was worthy of consideration. Having reviewed a range of commercially available frames designed for animal stereotaxy, a number of problems limited their use for our purposes.

Firstly, we utilized in our series of acute lesioning experiments a number of older Macaques. Their age was commensurate with an increase in their size, and most frames do not have the physical dimensions to be utilized in a safe and accurate manner on animals with a head circumference such as theirs.

Secondly, all available frames are constructed in metal - brass, aluminum or steel. Therefore all are radio-opaque. This presents a problem for targeting the PPN using ventriculography as with the primate's head positioned in a frame such that the orbito-meatal line lies parallel to the basal plane of the frame the area to be targeted may be obscured by the overlying metal side bar of the frame. Thus accurate and reproducible targeting was not possible by this means.

Therefore we proceeded to design and construct a frame which would be suitable for our specific requirements. 


\section{INTRODUCTION TO STEREOTAXIS}

Stereotaxis, from the Greek meaning 'three-dimensional, orderly arrangement', is based upon the principle that any point within a contained space (the brain in this instance) can be referenced to a specific co-ordinate system using precise measurements. The concept of a Cartesian co-ordinate system is based upon principles developed in the early seventeenth century by the French mathematician and philosopher Rene Descartes (Descartes 1637) and continues to form the basis for the majority of modern stereotactic frame designs. Descartes proposed that any point in space can be defined by its relationship to three planes, traditionally labeled $x, y$ and $z$, which intersect at right angles to each other. These three planes, the abscissa, ordina and applicata, meet at one point which is denoted the origin or zero point. Any point in space can therefore be defined by its distance as measured from the three reference planes. Utilizing the Cartesian co-ordinate system in animal stereotaxis, a midsagittal plane is traditionally used a one reference while a basal plane, perpendicular to the midsagittal plane, forms a second plane. Historically, the basal plane passed through the external auditory meatus and the lower borders of the orbits - the orbito-meatal plane - which is similar to the Frankfurt plane used by anthropologists in their measurements of the skull. The practical use of stereotaxis required the elevation of this plane by $10 \mathrm{~mm}$ in order to included the base of the brain. The third plane also transects the EAM orthogonal to the midsagittal and basal planes. The Cartesian coordinate system has withstood the test of time and is the method employed in modern frame design. Indeed this was the method employed by us in a translational or rectolinear fashion for the targeting of our radiofrequency lesions within the PPN, as has been described in the preceding chapter. 


\section{ANIMAL STEREOTAXIS}

The driving forces behind the development of systems of stereotaxy for use in animal experimentation in the early twentieth century were the same two individuals who later pioneered the techniques as applied to humans; Sir Victor Horsley a prominent British neurosurgeon and Richard Henry Clarke a Victorian scholar.

In their landmark publication of 1908, ' The structure and function of the cerebellum examined by a new method,' (Horsley \& Clarke 1908) they described their apparatus and its applicability in animal neurophysiological experiments. The impetus to develop this structure was Horsley's desire to study the function of the deep cerebellar nuclei. He recognized the importance of making exact lesions or performing localized stimulations in the deep tracts and nuclei without interfering with surrounding structures. The credit for the frames design, however, is attributed to Clarke who realized the futility of relating intracranial structures to external bony landmarks in the primate, and he based his system on Cartesian co-ordinates. The original frame, called 'Clarke's stereoscopic instrument employed for excitation and electrolysis' was constructed in 1905 by James Swift of Palmer \& Company in London at a cost of $£ 300$ (Fodstad et al 1991). The apparatus consists of a brass frame applied to the animal's head with bars attached to plugs inserted into the EAM and other bars resting upon the nose and orbital margins. Laterally placed pins are used to fix the frame to the skull. The instrument was first used in 1906 to make electrolytic lesions in the cerebellum of the Macacus mulatta (Schurr \& Merrington 1978). Although the investigator's conclusion that the cerebellar cortex is practically inexcitable was later disproven, their work was the first to employ electrolysis in experimental physiology (Jefferson 1957). Horsley and Clarke not only pioneered the stereotactic apparatus in animal neurophysiological experiments but also formulated the first stereotactic brain 
atlas (Horsley \& Clarke 1908), which was later reproduced as a separate monograph (Clarke 1920).

The implementation of stereotaxy in animal neurophysiological experimentation has continued to the present day along similar lines to those set down by Horsley and Clarke in the early part of the century. Modern frames for animal stereotaxy, such as the Kopf frame, are made of brass or aluminum and employ the Cartesian system of co-ordinates for targeting in a translational or rectolinear manner, using contrast ventriculography or external bony landmarks in non primates. Further advances which have been made this century in the field of human stereotaxy (e.g. arc systems and frameless stereotaxy) are dependant on imaging techniques, CT and/or MRI, which are not widely available for use with animals. Therefore animal frames have not benefited from these advances.

\section{MATERIALS AND DESIGN}

Ideally, any stereotactic instrument should be extremely accurate and practical in any position yet simple to use in order to minimize operator error. Our procedure required a stable, reliable and versatile stereotactic frame that would permit access below the level of the plane of the frame itself if necessary. These requirements of a stereotactic frame had been addressed in the clinical setting of human stereotaxy in the 1960's by the British neurosurgeon Edward Hitchcock ( Hitchcock 1969). Hitchcock had originally developed his frame for use in his procedure of high spinal stereotactic commisural myelotomy, however, the Hitchcock frame continues in use in some centers today, following only minor modifications to the original design, for many functional and stereotactic procedures. The basic components of the Hitchcock frame are; the square and the L-grid, with targeting utilizing a classical Cartesian coordinates system. It was these principles which we employed in the design of our sub-human primate frame. 
The basic component of our frame consists of an open fronted rectangle, rather than square, head frame. This head frame is fashioned from a single piece of extruded acrylic-clear (Perspex), cut on a milling machine with an error margin of $0.1 \mathrm{~mm}$. Perspex has a flexural strength of $105.0 \mathrm{~N} / \mathrm{mm}^{2}$ and a tensile strength of $75.0 \mathrm{~N} / \mathrm{mm}^{2}$ it is therefore well able to support the weight of a monkey's head without any bending occurring. Its light transmission coefficient is $92 \%$ therefore it is almost completely radiolucent and so allows for targeting of areas which may lie posterior to the side arms of the frame. The external dimensions of the frame are $204 \mathrm{~mm} \times 160 \mathrm{~mm}$, with internal dimensions of $179 \mathrm{~mm} \times 108 \mathrm{~mm}$. Anteriorly the frame is open and attaches rigidly to the operating table via two laterally placed, inverted L-shaped struts also machined from Perspex. The fixation is via locked bolts and Tensol glue, and is such that the frame lies parallel to the plane of the operating table at a height of $259 \mathrm{~mm}$ above the table. Fixed between the anterior Perspex struts is the adjustable mouth-plate and eye-bars, which allow for anterior fixation of the animals' face within the frame (Figs. 3.1a,b,c \& 3.2).

Rigidly attached to the lateral aspect of the right hand side arm of the frame, a distance of $25 \mathrm{~mm}$ from its anterior edge, is the mounting for the microdrive which allows for target co-ordination in our three Cartesian planes $x, y$ and $z$. Midway along each frame sidearm (i.e. at $102 \mathrm{~mm}$ from either end), centered on the midpoint for thickness (i.e.13mm from either edge), are machined full thickness holes of diameter $6 \mathrm{~mm}$. These holes serve for the insertion and fixation, by means of a superiorly placed plastic screws, of the earbars which are to support and position the animals head into the midpoint of the frame (Fig 3.2). The earbars are machined from Perspex on a lathe to a diameter of $6 \mathrm{~mm}$ (error of margin $0.1 \mathrm{~mm}$ ), and have inserted a central core of lead throughout their length, this core is to assist in obtaining $\mathrm{X}$-rays in the true sagittal plane as both should overlap in this case. On the upper surface of each earbar there are machined ruling marks of a scale in 1,5 and $10 \mathrm{mms}$ these are 
to allow for accurate measurement of the animals' head from either side of the frame, thus ensuring it to be placed centrally. These markings are overlaid using a silver loaded micro-tip circuit works pen thus ensuring that they are easily visible for reading. Also, the hole in the right sidearm allows for the insertion of a 'zeroing pin'. This is a Perspex cylinder of similar dimensions to the earbars and machined in an identical fashion, which at its medial end has rigidly attached an L-shaped lead pin whose tip points upwards. The cylinder is so fashioned that when correctly placed within the sidearm hole it may be securely fixed by means of a locking screw from the inferior aspect of the frame. In this position, the tip of the attached lead pin lies at the center point of the frame, this is our zero point to be used for further target calculations.

At a distance of $130 \mathrm{~mm}$ from the anterior edge of each side arm and placed centrally for arm depth, there is etched into the Perspex a crosswires figure. These have then been overlaid using the silver loaded microtip circuit works pen thus rendering them radio-opaque. These serve as a further aid to ensuring true laterality of an X-ray, as again they should be congruous in a truly lateral film.

At the midpoint of the posterior limb of the frame is attached a $50 \mathrm{~mm}$ wide Perspex block into which may be secured our L-grid. This is an L-shaped ruler cut from Perspex which is etched in divisions of $1,5,10 \mathrm{~mm}$ with the etchings again being overlaid by a silver loaded pen to render them radio-opaque. This grid is attached to the block on the posterior limb of the frame and included in our template X-ray in order that the scale may be used to assess the magnification on X-ray by its comparison with a non-magnified rule (Fig 3.3). 
Figure 3.1. Views of our Stereotactic Frame with the Zero Sidearm in Place from; a.Superior
b.Lateral
c.Posterior

a.Superior

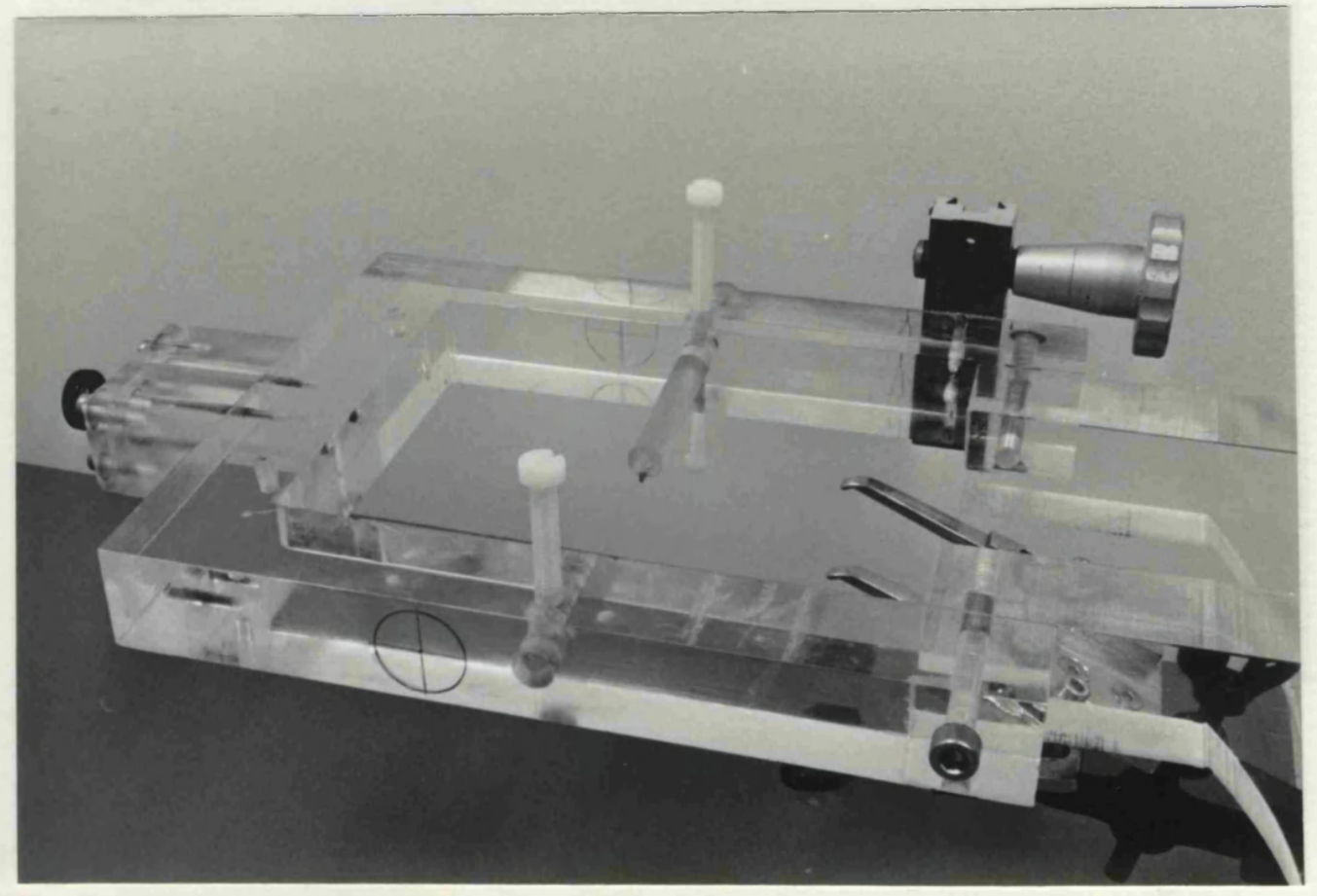

b.Lateral

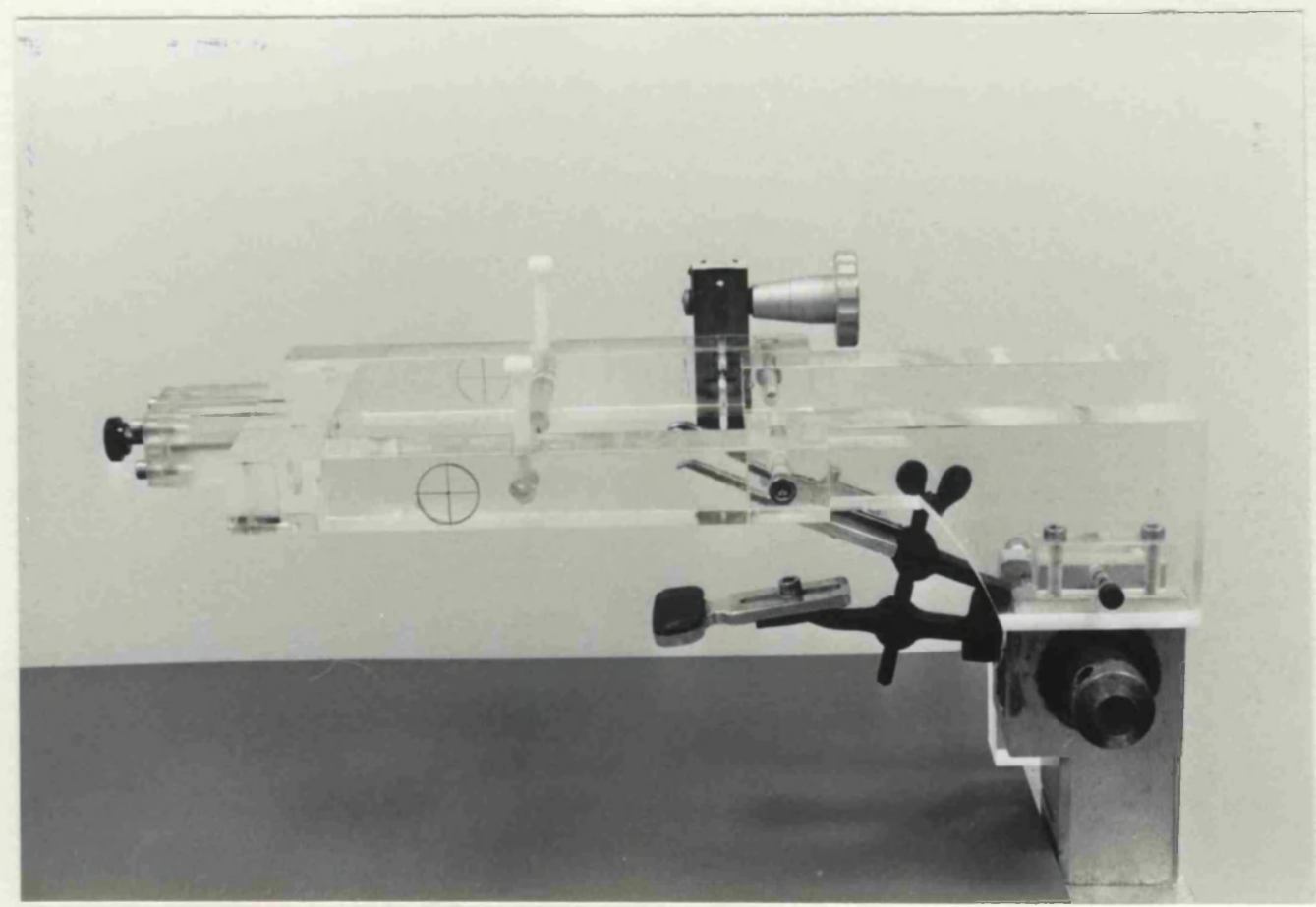


c.Posterior

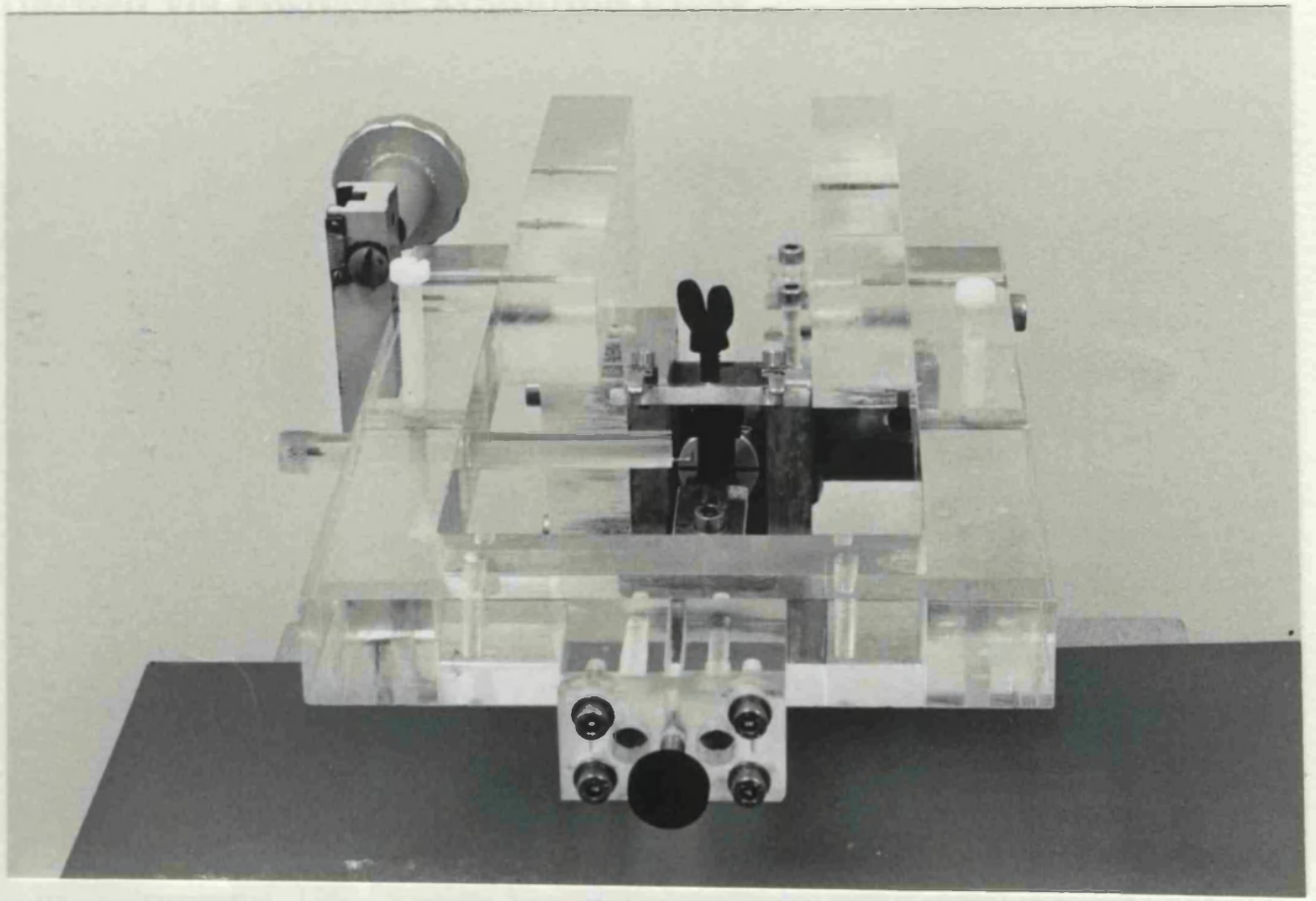

Figure 3.2. Superolateral view of our frame containing a Monkey Skull to Demonstrate Fixation by the Earbars, Mouthplate and Infraorbital Bars.

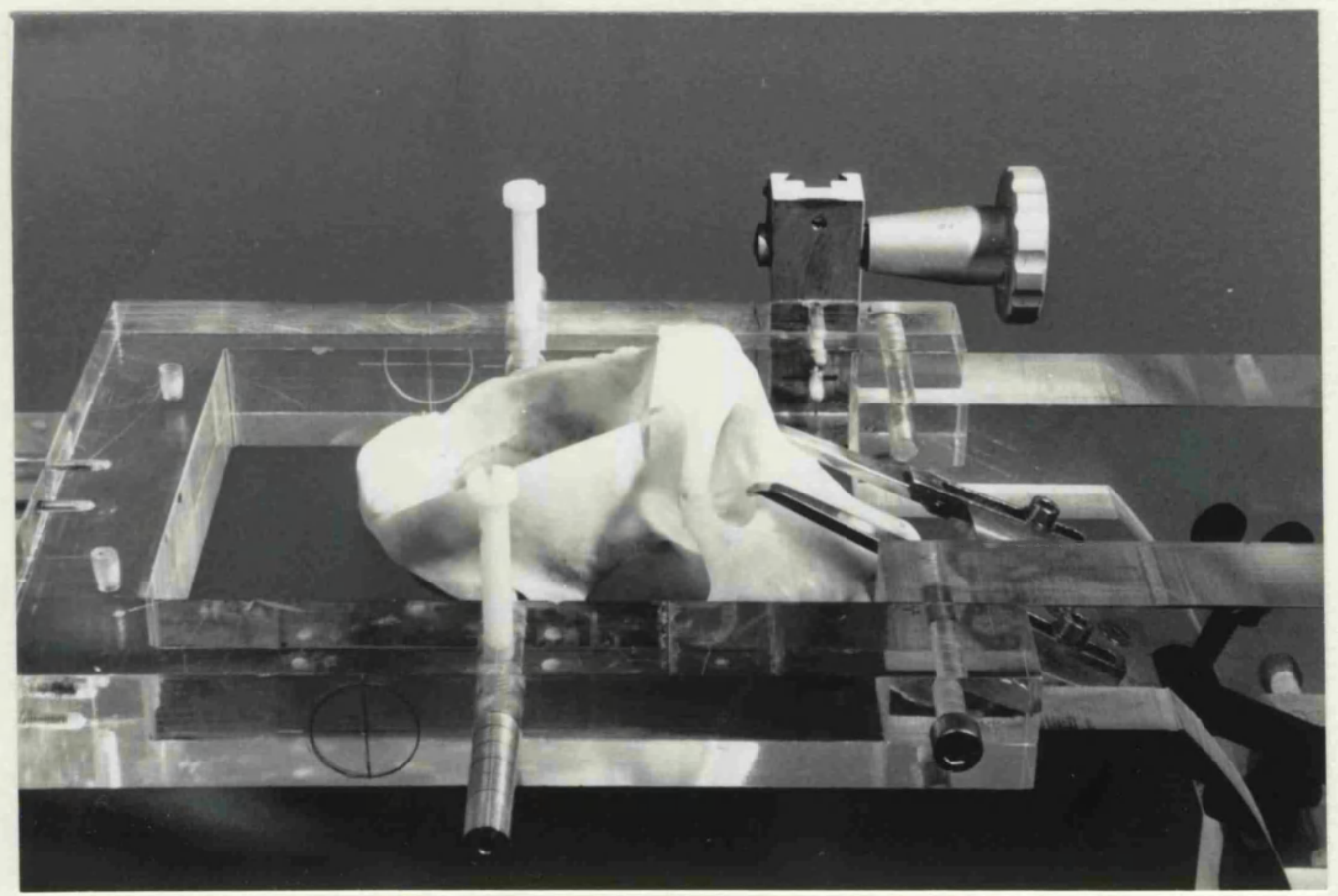


Figure 3.3. Lateral View of Frame with L-Shaped Scaling Rule Attached via Posterior Mounting Block.

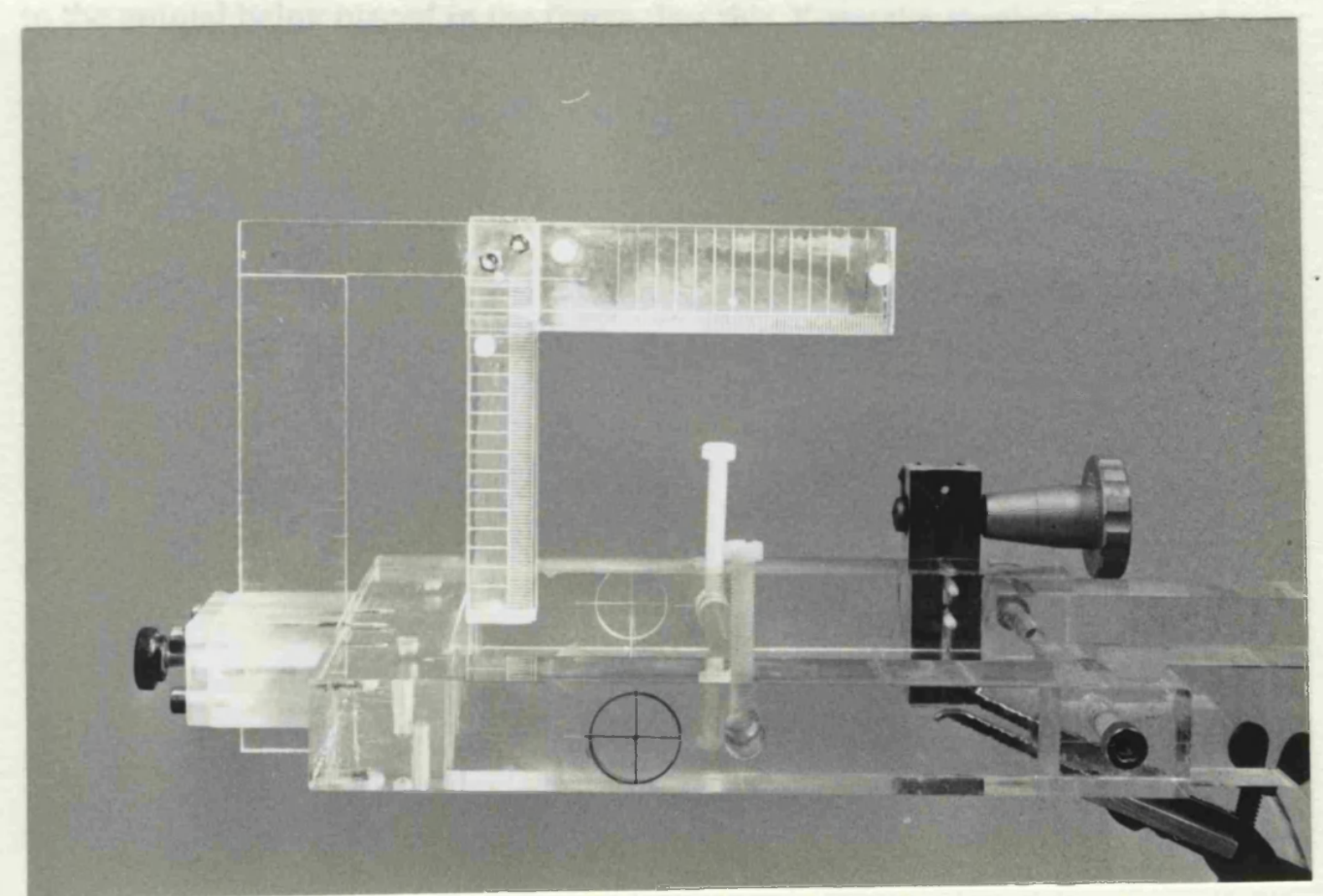




\section{TARGET ACQUISITION}

The use of our frame necessitates that an initial template X-ray be taken prior to the animal being placed in the frame. For this X-ray the zeroing pin must be correctly fixed in position and the L-grid scale rule also be included. On such an X-ray it is possible to assess the plane of the film, and the magnification factor for X-ray (Table 3.1). Also at this time it is necessary to obtain the coordinates for our zero point. In order to do this the Hamilton syringe which is to be used for later injection of the kainic acid must be fixed in the holder attached to the microdrive of the frame. The Hamilton needle tip is now brought to lie exactly tip-to-tip with our frame zero pin (Fig. 3.4a,b). In this position the co-ordinates in all three planes must be recorded - $x, y$ and $z-$ these are now our zero co-ordinates.

Animals must be positioned within the frame such that the head lies centrally, as measured by the distance from the frame on the calibrated earbars which have been inserted into the EAM. Anteriorly, fixation is by means of a steel mouthplate positioned beneath the maxilla in the mouth, and a pair of eyebars which should lie on the infra-orbital ridge bilaterally, these two mechanisms attach anteriorly to form a pincer like fixation device (Fig3.2).

Following the acquisition of a contrast ventriculogram, and proportional correction for size and magnification and reference to a standard atlas, as will be discussed in detail later, the site of the target PPN may be marked on the ventriculogram as relative to the posterior commisure (PC). The ventriculogram is then overlaid onto the original template $\mathrm{X}$-ray and the zero point marked on the ventriculogram. Thus a direct measure of the target site in relation to our previously triangulated zero may be made in the AP and DV 
planes. The co-ordinates of laterality having been calculated by proportional correction for size and atlas dimensions (Table 3.1).

The method of targeting outlined is that which we proceeded to employ for our series of cytotoxic lesions as shall follow (Fig. 3.5).

Figure 3.4. Anterior Views of the Hamilton Syringe in the Side Mounted Microdrive, Zeroed Tip-to-Tip with the Zeroing Pin. a.Shows Hamilton in Microdrive b.Close up of Zeroed Needle Tip

a.

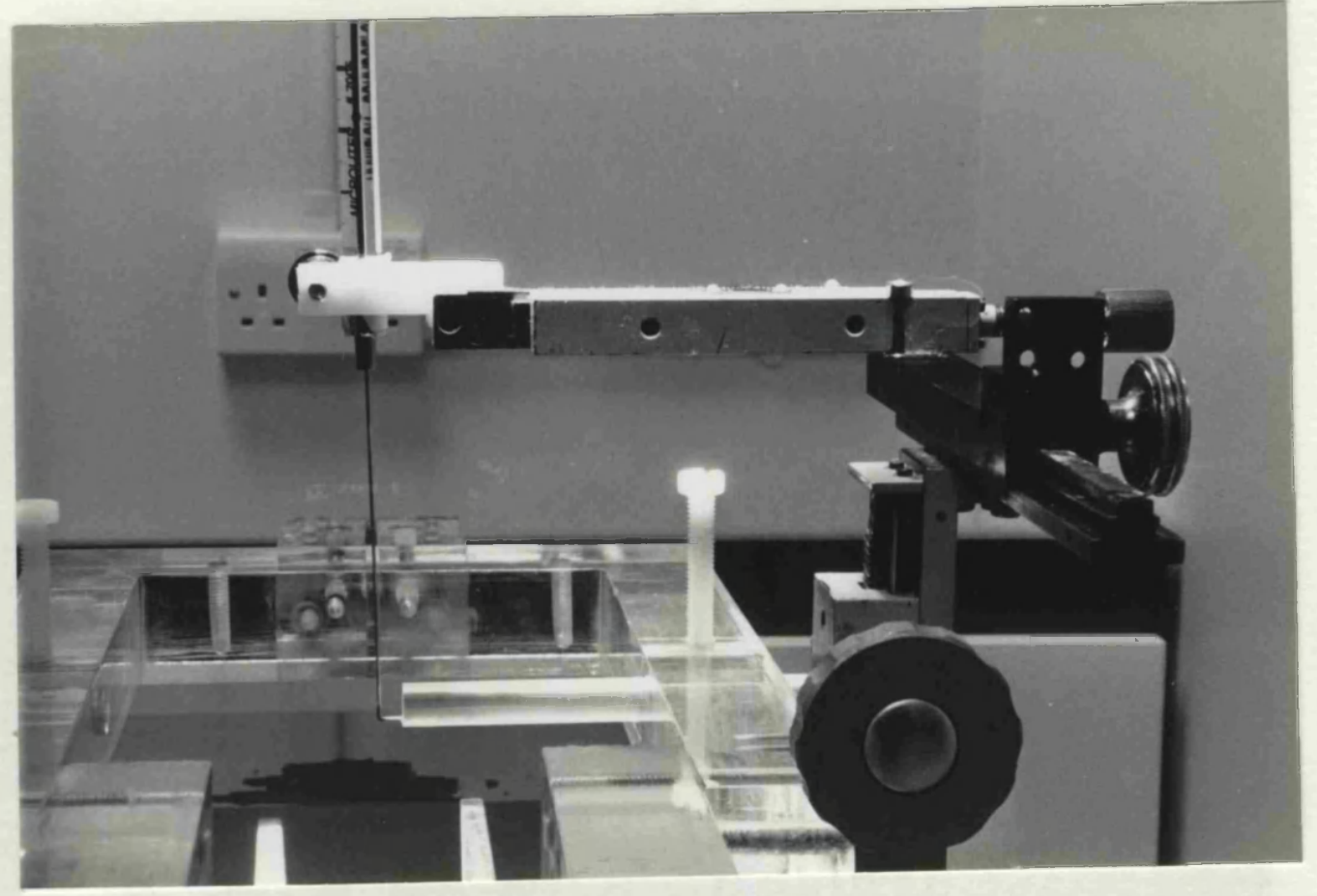


b.

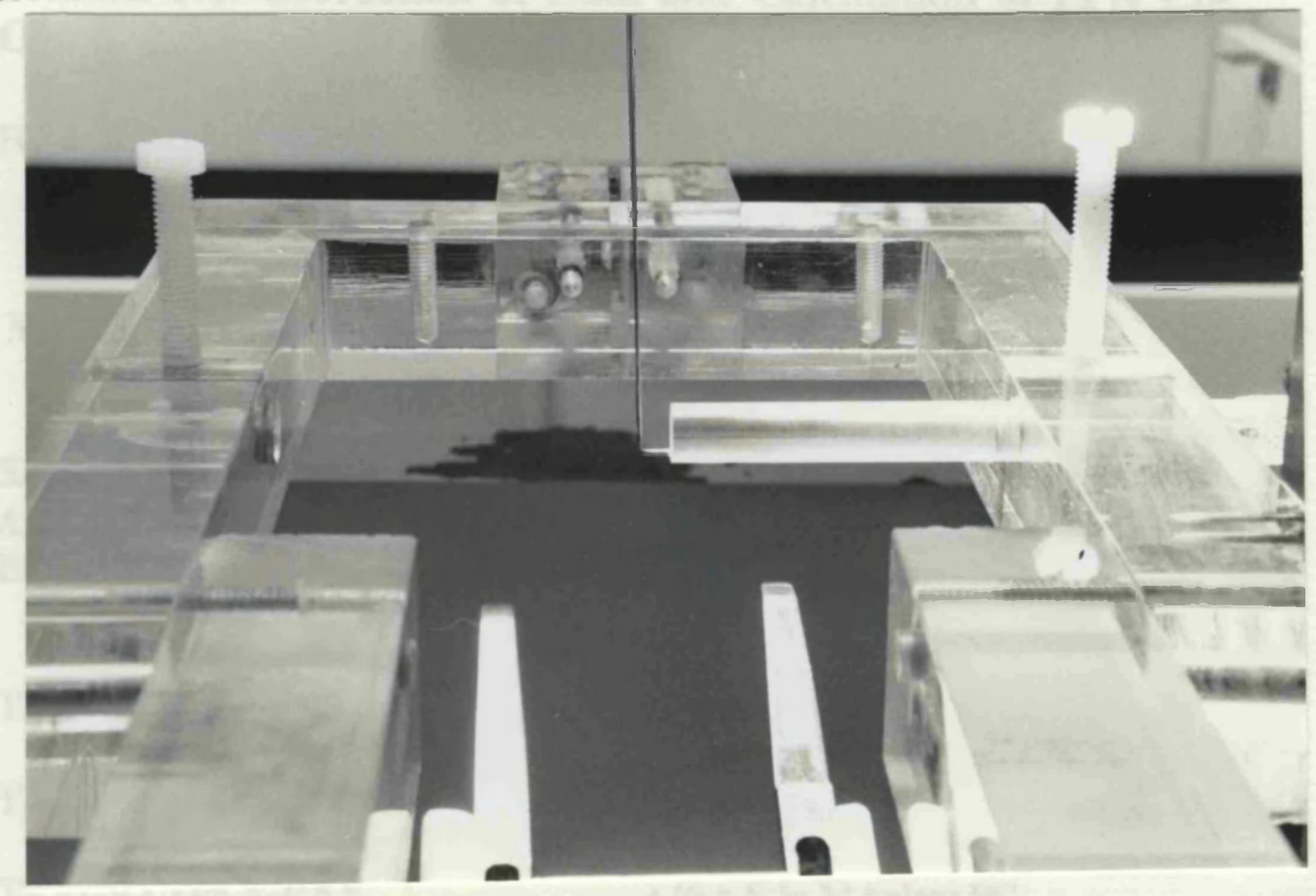

Figure 3.5. Lateral View Demonstrating Positions of Microdrive and Hamilton at Target.

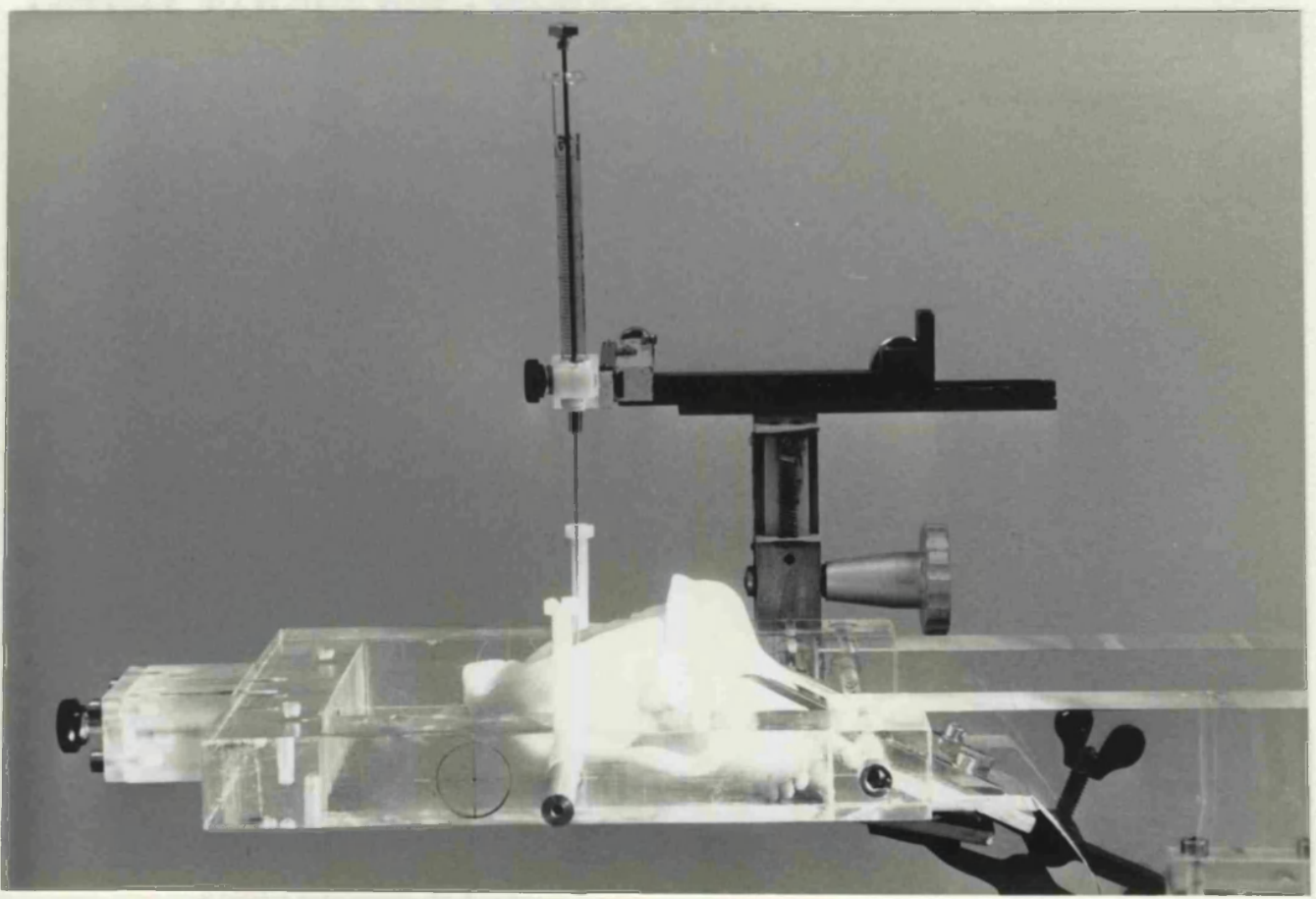


TABLE 3.1. Correction of Data and Calculation of PPN CoOrdinates in Cytotoxic Lesioning.

HAMILTON ZERO : AP $=x$

Lat $=y$

Vert $=z$

MAGNIFICATION : $5 \mathrm{~mm}$ on graph $=\alpha \mathrm{mm}$ on X-Ray scale $\alpha / 5=$ magnification factor $(\mathbf{M})$

X-RAY AC-PC DISTANCE $[\mathrm{cm}] \quad: \beta$

ACTUAL AC-PC DISTANCE[cm] $\quad: \beta / \mathrm{M}=\mathrm{E}$

ENLARGEMENT FACTOR OFF ATLAS: E/1=E (as atlas AC-PC $=1 \mathrm{~cm}$ )

TARGET FROM ATLAS $\quad: 6 \mathrm{~mm}$ below PC

$2 \mathrm{~mm}$ lateral to midline

TARGET CORRECTED OFF ATLAS : $6 \mathrm{x}$ E below PC

TARGET ON X-RAY

$2 \times$ E lateral to midline

: $(6 \times$ E $) \times$ M below PC

$(2 \times$ E) lateral to midline

TARGET RELATIVE TO ZERO ON X-RAY:AP $=\chi$ ANTERIOR to zero

Vert $=\delta$ SUPERIOR to zero

ACTUAL TARGET RELATIVE TO ZERO:

$\mathrm{AP}=\chi / \mathrm{Mmm}=\mathrm{Amm}$ ANTERIOR/POSTERIOR to zero

Vert $=\delta / M=D$ SUPERIOR/INFERIOR to zero

\section{RIGHT CO-ORDINATES $\quad$ LEFT CO-ORDINATES}

AP

$\mathrm{x}+/-\mathrm{A}=\mathbf{A P}$

Lat $\quad \mathbf{y}+(2 \times E)=$ RLat

$y-(2 \times \mathrm{E})=\mathbf{L L a t}$

Vert

$$
z+/-\mathbf{D}=\mathbf{D V}
$$

In AP plane ANTERIOR $=-\mathrm{A}$

In VERT plane SUPERIOR $=+\mathrm{D}$

POSTERIOR $=+\mathrm{A}$

INFERIOR $=-\mathbf{D}$ 


\title{
CHAPTER FOUR
}

\author{
EXCITOTOXIC LESIONS OF THE \\ PEDUNCULOPONTINE NUCLEUS IN THE \\ NORMAL SUB-HUMAN PRIMATE
}




\section{EXCITOTOXIC LESIONS OF THE PEDUNCULOPONTINE NUCLEUS IN THE NORMAL SUB-HUMAN PRIMATE}

\section{INTRODUCTION}

Having established in our earlier studies with radiofrequency lesions that a parkinsonian like akinesia can indeed be generated by making lesions of the pedunculopontine region of the normal sub-human primate, our aim in this second part of the study was to further refine and reconfirm these findings.

The lesions generated by the radiofrequency method were large in relation to the structure targeted, having a diameter of $1-2 \mathrm{~mm}$, and although staining with Luxol fast blue (LFB) confirmed that fibres of the superior cerebellar peduncle remained intact, the question remained as to whether some of the observed effects could have resulted from damage caused to surrounding fibers of passage.

With this question in mind our aim was to create a neuron specific lesion using the excitotoxic agent kainic acid, thereby eliminating the possibility of any effects being mediated through damage to surrounding fibers of passage. These lesions were created utilizing our redesigned stereotactic frame in an effort to maximize our accuracy. Should we once again generate a selective, parkinsonian akinesia by these methods this would add further support to our hypothesis that the PPN plays an important part in limb movement and locomotor control and the aetiology of parkinsonian akinesia. 


\section{MATERIALS AND METHODS}

Seven further macaque monkeys were used in this study (4 female Macaca mulatta, 3 male Macaca fascicularis), their mean age was 7 years (range $4-17$ years), and mean weight was $5.6 \mathrm{~kg}$ (range $3-8.4 \mathrm{~kg}$ ). They were housed in accordance with the Home Office code of practice for the housing and care of animals used in scientific procedures, with all animals housed in a cage of dimensions suitable for those in the $6-9 \mathrm{~kg}$ category, i.e. Minimum floor space $14,000 \mathrm{~cm}^{2}$, with a minimum height of $150 \mathrm{~cm}$. A fixed 12 hour light dark cycle (07:30 - 19:30hrs lights on, 19:30 - 07:30hrs lights off) was adhered to throughout, and ad lib food and drink provided at all times, except on the morning prior to surgery. Under these circumstances no animals exhibited any signs of environmental stress.

Mounted above the home cage was a Doppler activity meter that covered a conical field encompassing the cage area. The meter's sensitivity was set so as to require whole limb or whole body movements to register a single count, the objective being to record the overall activity of the animal during each 24 hour period. The gain of the activity meter was checked and adjusted for each monkey to account for the variation in physical size between animals. Counts were taken at the same time daily to represent a total count for the preceding 24-hour period. Activity monitoring by this method was performed for a minimum period of 7 days prior to surgery in order to provide a baseline 24hour activity count for each animal (N.B. Pre-operative activity monitoring was only commenced once the necessary gain adjustments had been established for each individual animal). 
In addition to the recording of daily activity counts, all animals were assessed clinically by scoring them on the clinical rating scale to assess Parkinsonism in the primate (Table 4.1, Clarke 1987). Within this system 10 parameters are assigned a 3-5-point scale of severity. All parameters scored are those which can be observed without disturbing the animal, thus rigidity is not formally scored but may be assessed in the home cage by passive resistance of the limbs to movement. The animals could thus attain a maximum score of +16 for severe parkinsonism, whilst a score of zero represents normal behavior. Within this system a negative score of up to a maximum of -8 is possible, this facility allows for the assessment of MPTP treated animals receiving over treatment with dopamine or dopamine agonists and was therefore not applicable to our study. Scoring was performed by the same individual in all cases both pre and postoperatively in order to exclude the possibility of any observer bias. Clarke's scores were performed daily on each animal preoperatively, and daily from days 3 to 7 post operative in order to allow a 48hour recovery period prior to assessment. Both observed and unobserved activity was considered when scoring each monkey.

Video recordings of both provoked and unobserved activity were performed to provide a record of speed and nature of movements, facial expression and responsiveness to changes in the surrounding environment. In some instances periods of unobserved recording extended into the animal's 12-hour dark cycle period, however, analysis of sleep behavior patterns has not been included in our present analysis. 
Table 4.1. The Clinical Rating Scale Used to Assess

Parkinsonism in Monkeys (Clarke 1987)

\section{ITEM}

Activity

\section{$\underline{\text { SCORE }}$}

$-2$

$-1$

0

1

2

Bradykinesia

Freezing

Balance

Posture

Tremor

2

Feeding

Vocalisation

0
1
2

Chorea

\section{0}

$-1$

$-2$

Stereotypy

\section{DEFINITION}

Severe hyperactivity Moderate hyperactivity

Normal

Moderate hypokinesia

Severe hypokinesia

Normal

Moderate bradykinesia

Severe bradykinesia

None

Some episodes

Many episodes

Normal

Moderately impaired

Severely impaired

Normal erect

Some stooping

Severe Stooping

None

Moderate tremor

Severe tremor

Hyperphagia

Normal intake

Reduced intake

No intake

Hyperphonia

Normal

Reduced

Markedly reduced

None

Moderate

Severe

None

Moderate

Severe

Total

-8 to +16 
All methods of assessment described were performed in an identical manner both pre and post operatively, all being performed by the same assessor in all instances.

As discussed in Chapter 2 (Materials and Methods) in large sub-human primates it is not possible to utilize bony landmarks for intracranial target acquisition as is practiced in rodents and cats. Therefore, for the reasons previously outlined, targeting was once again based on contrast ventriculography with proportional correction for size and X-ray magnification. To account for the magnification secondary to a divergent Xray beam we included a linear scaling arm marked in mms in our frame design. On each occasion a 'template' $\mathrm{X}$-ray was performed with the scale arm in situ, by comparison of a 5mm measure on the magnified X-ray to an actual $5 \mathrm{~mm}$ measure on transparent graph paper marked at $1 \mathrm{~mm}$ intervals, the X-ray magnification fraction can be assessed and corrected for on each occasion. Also, the template $\mathrm{X}$-ray was used to assess the position of our frame central 'zero point' in readiness for target calculation.

Prior to surgery the Hamilton syringe, to be used for the later injection of the kainic acid, is placed in the horizontal holder of the side arm of the frame. This is attached to a calibrated drive which is able to make movements calculable to $0.1 \mathrm{~mm}$ in all three planes. This is then positioned with the tip of the syringe needle positioned tip-to-tip with our frame central, 'zero indicator'. In this position the co-ordinates of the microdrive in the AP, lateral, and DV planes ( $x, y$ and $z$ ) are recorded for our later target calculations, all of which are performed as relative to our frame central zero point. 
The animals were fasted on the night prior to surgery. On the day of surgery all animals were initially sedated with a single dose of intramuscular ketamine (10mg/kg-body weight). Thereafter anaesthesia was maintained by means of a continuous intravenous infusion of althesin titrated according to the animals' weight $(6 \mathrm{mg} / \mathrm{kg} / \mathrm{hr})$, and administered via an infusion pump. When anaesthetised the animals head was shaved prior to being placed in our stereotactic head frame. They were positioned within the frame so that the distance between the supportive ear bars and the frame was identical on either side. Anteriorly the skull was immobilized by means of a mouthpiece placed under the maxilla and hard palate inferiorly, and superiorly a pair of eye bars which fixed to the inferior orbital ridge on either side of the animals head. With this fixation the orbito-meatal line lay parallel to the horizontal sidebars of the frame. Having positioned the animal thus, a check X-ray was taken to ensure that the sagittal plane of the animal lay perpendicular to the X-ray beam.

All surgery was performed under aseptic conditions, and all animals received intramuscular antibiotic prophylaxis (Synulox $0.06 \mathrm{ml} / \mathrm{kg}$ ) on the day prior to surgery, on induction of anaesthesia and for 2 days post operatively. The skin was prepared with an alcohol/iodine solution and a linear incision sited over the sagittal suture. In order to perform a ventriculogram a burr hole was made at a point $1.5 \mathrm{~mm}$ lateral to the midline, and $20 \mathrm{~mm}$ anterior to the external auditory meatus (EAM). Through this, a 23 gauge sterile needle was passed vertically into the anterior horn of the lateral ventricle, as confirmed by the aspiration of cerebrospinal fluid (CSF), usually at a depth of $15-20 \mathrm{~mm}$ dependant on the individual animal's size. Attached to the needle, and held in 
the microdrive arm of the frame, was a $1 \mathrm{ml}$ syringe containing contrast medium (Omnipaque 300, Nycomed UK). On free aspiration of CSF 0.5$1.0 \mathrm{ml}$ of Omnipaque was injected into the ventricular system and a lateral $\mathrm{X}$ ray taken (Fig 4.1).

Following contrast ventriculography and proportional correction for size with reference to a standard atlas (Shanta 1968) and magnification, a target was calculated in the PPN, $2.0 \mathrm{~mm}$ lateral to the midline and $6.0 \mathrm{~mm}$ below the posterior comissure. The method used for correction and target calculation is illustrated in Table 3.1 .

In order to site our lesion a second burr hole was made at the point on the skull which the Hamilton needle lay above when the AP and Lateral coordinates for the target were set on the side arm, thus allowing access at the appropriate point of the skull to set the DV co-ordinate for our calculated target. In the case of animals undergoing simultaneous bilateral lesions a single burr crossing the midline was made at this point. The dura beneath our burrhole was incised in order to prevent the possibility of any deviation of our injecting needle tip, and then the needle advanced through the incised area such that the required vertical setting for targeting be achieved with the AP and Lateral co-ordinates for target also set. At this point a further lateral radiograph was performed in order to confirm our targeting (Fig 4.2), on this $\mathrm{X}$-ray the position of the needle tip was again measured in comparison to our central frame zero. Lesions were made by pressure injection of 1-3microlitres of a $0.1 \%$ solution of kainic acid, dissolved in physiological saline, via a $0.5 \mathrm{~mm}$ diameter needle attached to a 1 or 5 microlitre Hamilton syringe, the needle tip being sited at the targeted PPN as confirmed on X-ray. Injection 
was made over a period of 5-10 minutes with gradual withdrawal of the injecting syringe over a further 5 minutes in order to minimize deposition of kainate within the needle tract secondary to any vacuum effect on withdrawal. In three animals a unilateral lesion was first made with a second contralateral lesion being made one week later. In a further three animals bilateral lesions were made during a single operation, and in one animal a unilateral lesion only was made. An intramuscular dose of buprenorphine $(0.06 \mathrm{ml} / \mathrm{kg}$ body weight) was administered at the end of each procedure to allow for postoperative analgesia. All animals were maintained on an intravenous hydration regime $(40 \mathrm{mls} / \mathrm{kg} / 24 \mathrm{hrs}$ Normal Saline) until they were able to safely maintain adequate oral hydration. The surgical incisions were closed with interrupted sutures of 3/0-coated Vicryl and the animals returned to the home cage where they were cared for on Vetbed and under an ultraviolet heat lamp for the initial $12-24$ hours post operatively.

Following recovery from anesthesia all animals were monitored in an identical fashion to that which had been applied pre-operatively (daily activity counts, Clarkes scoring and video recording) for a 7-day period. After this period the animals were sacrificed by means of an intravenous overdose of sodium pentobarbital (60mg/kg-body weight), and perfused transcardially with physiological saline followed by $10 \%$ formalin in a $0.1 \mathrm{M}$ phosphate buffer. The heads were then remounted in the stereotactic frame and the region of interest removed en bloc and placed in the same buffered formalin solution containing $30 \%$ sucrose. After a period of 7 days fixation in the sucrose/formalin solution, the tissues were cut serially into 50-micrometer 
sections on a freezing microtome. Alternate sections were then mounted and stained with Cresyl Violet to assess lesion placement and size (Fig $4.3 \mathrm{a}-\mathrm{e}$ ). 
Figure 4.1a. Lateral Ventriculogram of Monkey in Stereotactic Frame.

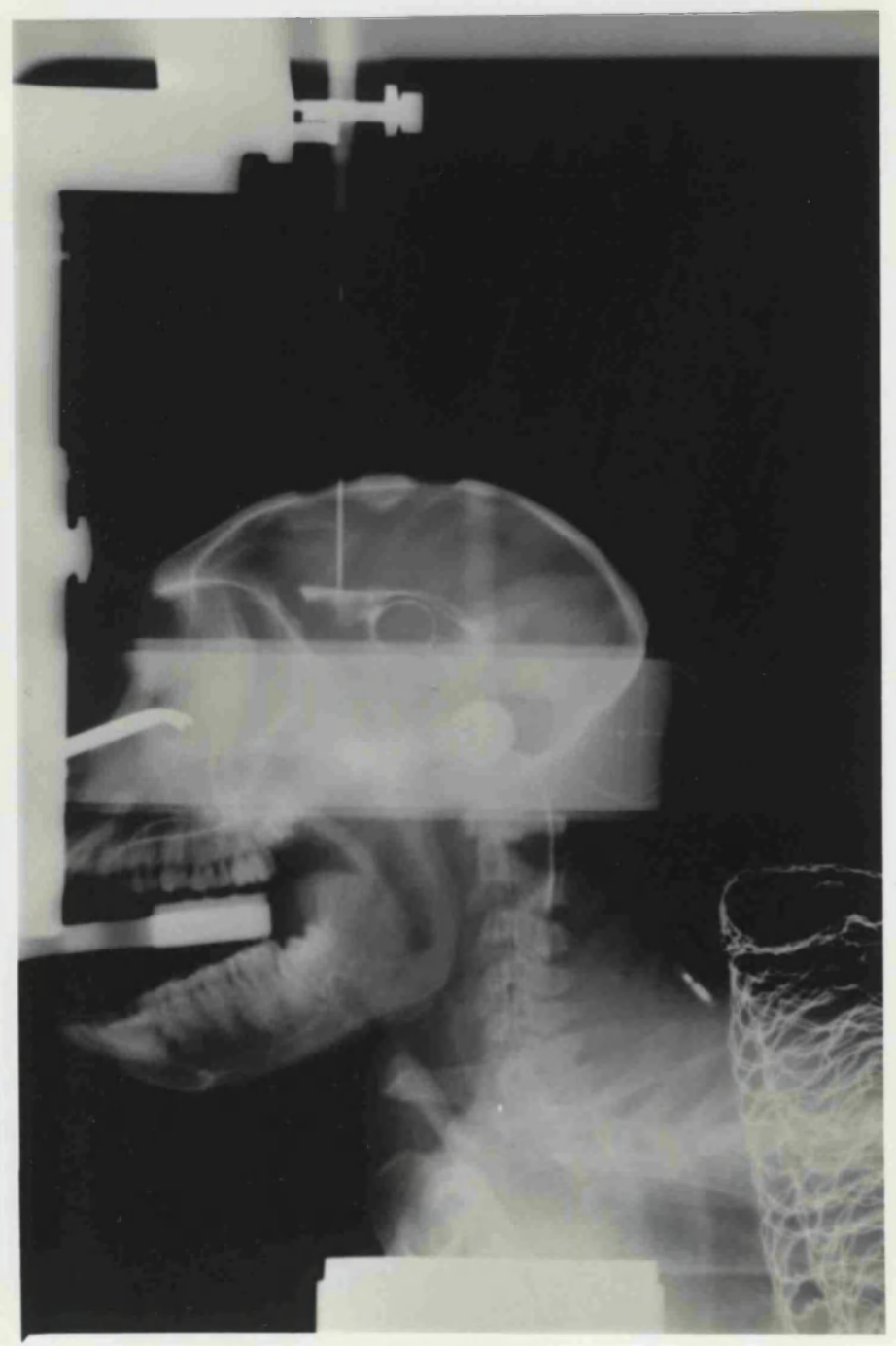

Figure 4.1b. Tracing of above Ventriculogram Identifying AC, PC and Target Site (LV - lateral ventricle, $3 \mathrm{~V}$ - third ventricle).

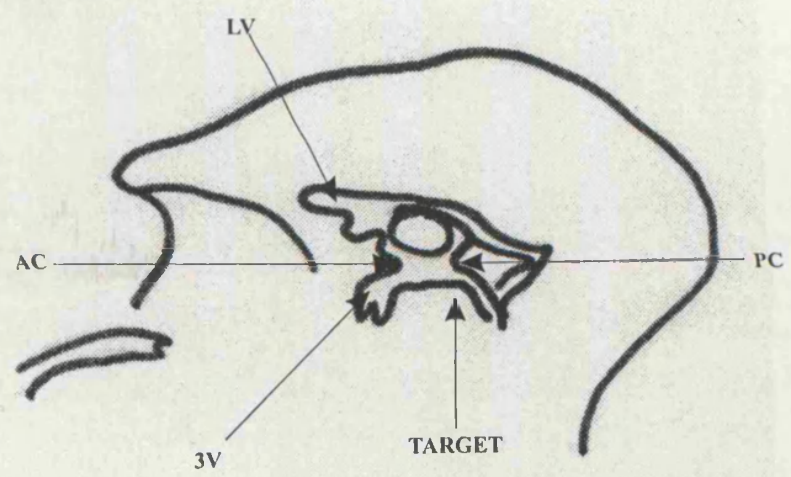


Figure 4.2. Lateral radiograph of Monkey in Stereotactic Frame with Hamilton Syringe Tip Sited at Target.

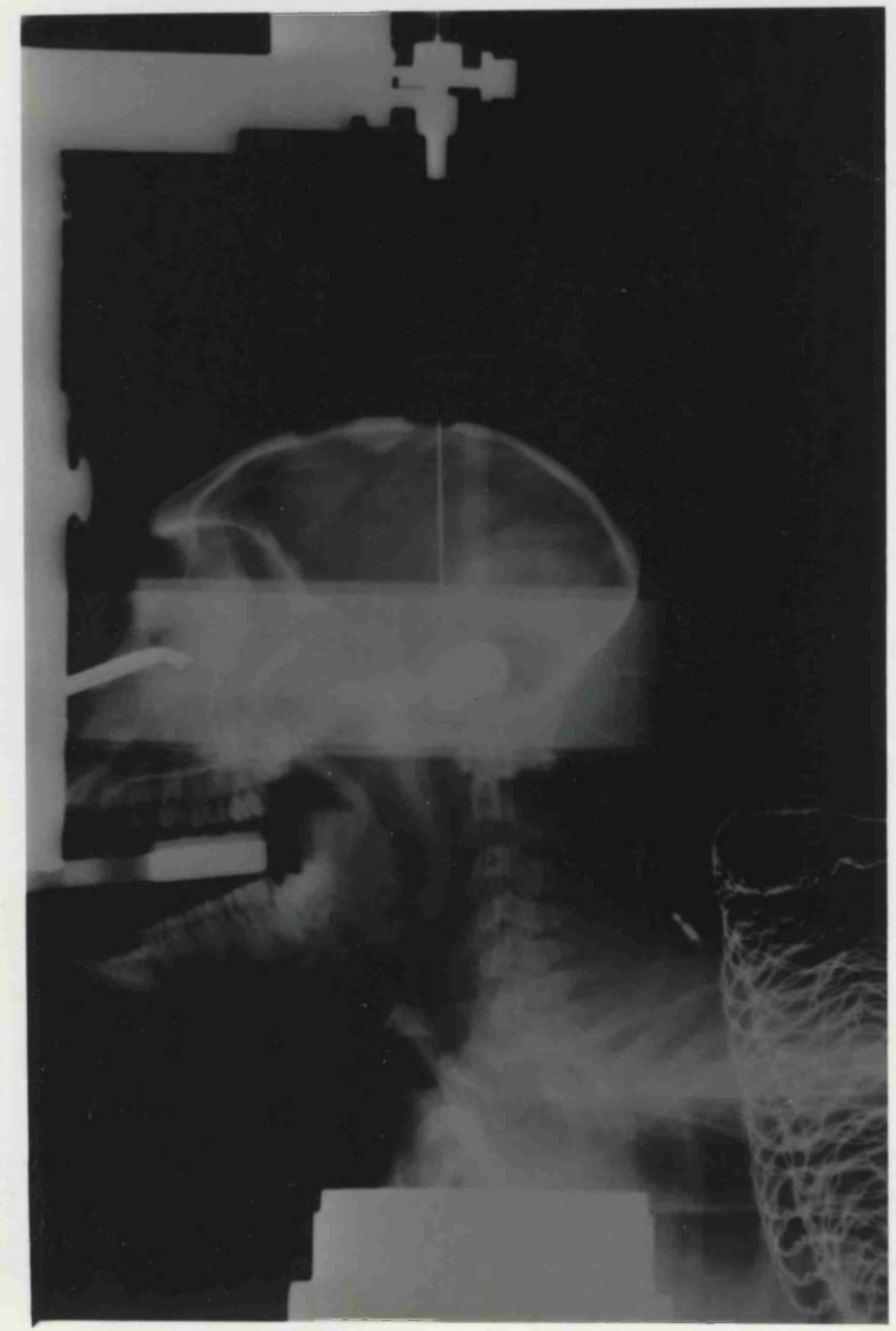


Figure 4.3. Histological Sections from Monkeys A - E with Corresponding Photomicrograph Illustrating Gliosis in the Lesion Region Adjacent to Arrowed Area. (Magnification of Photomicrograph $=\mathrm{x} 135$ ).

a.) Monkey A

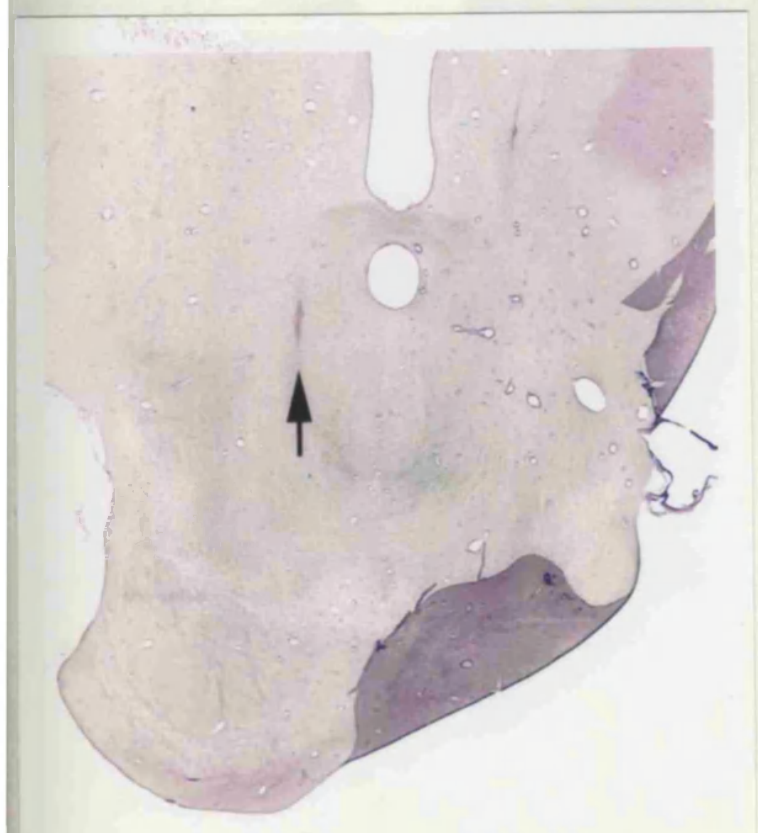

b.) Monkey B

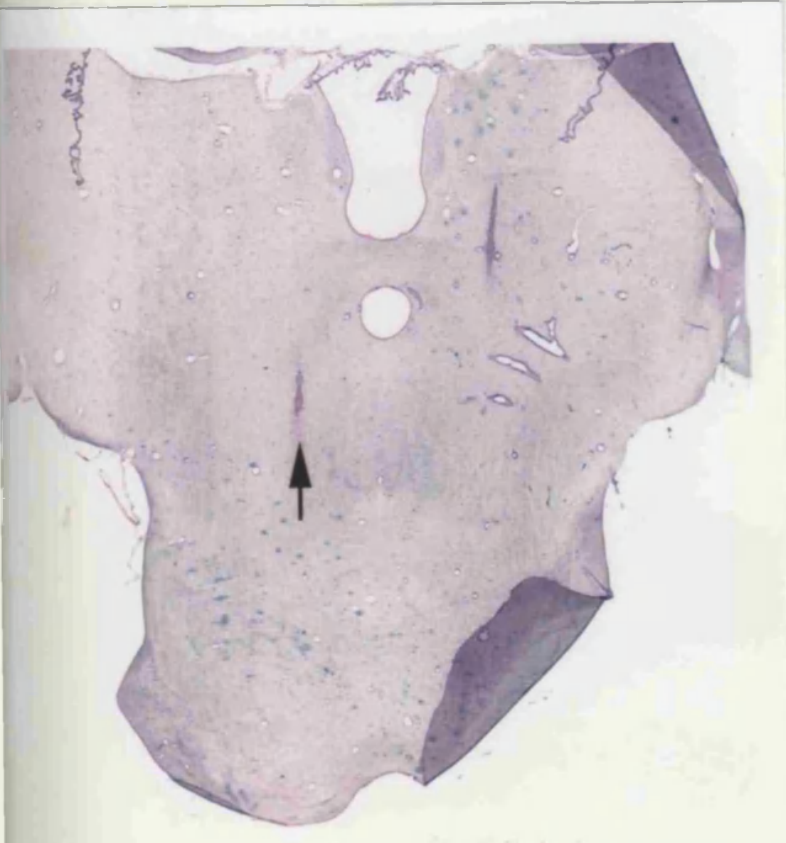

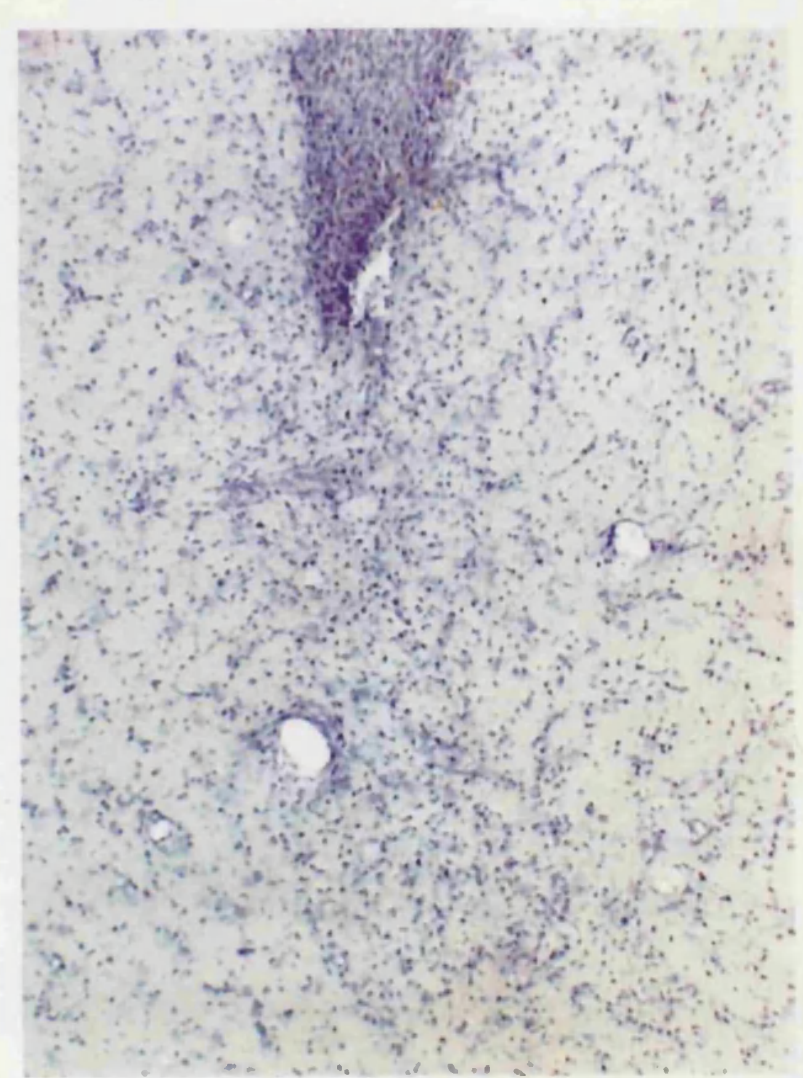

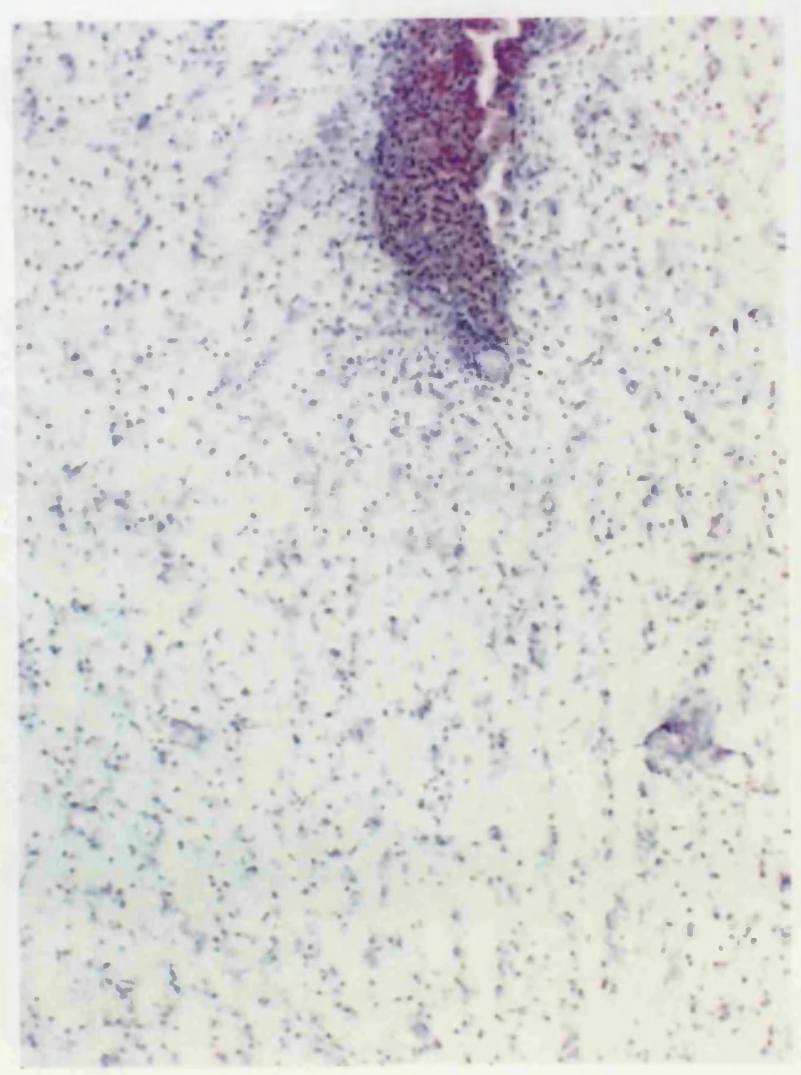


c.) Monkey C

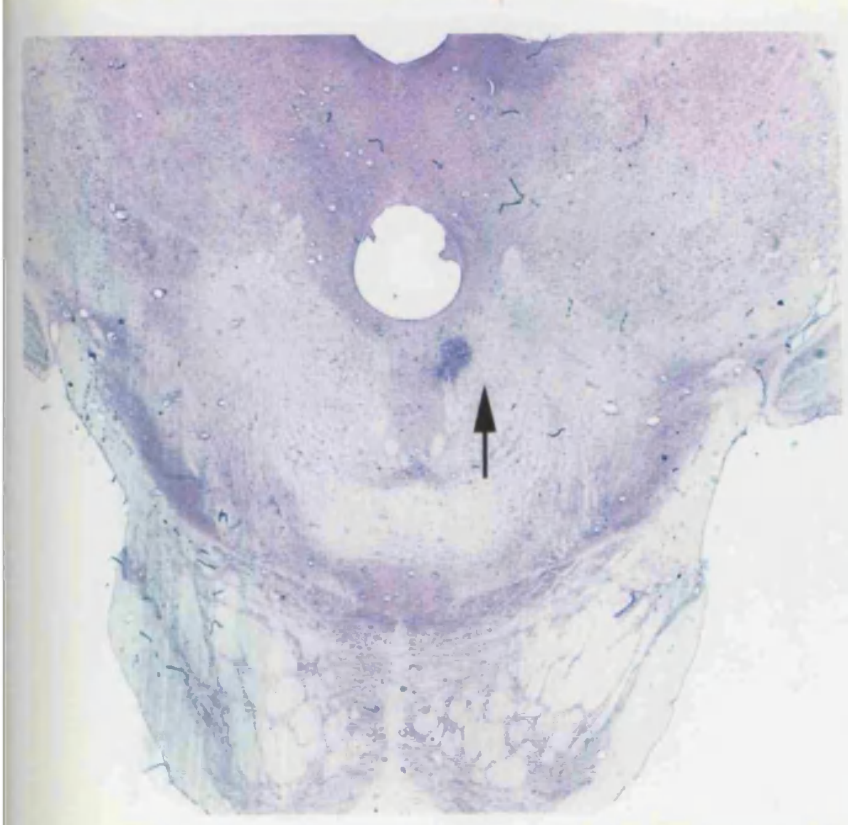

d.) Monkey D

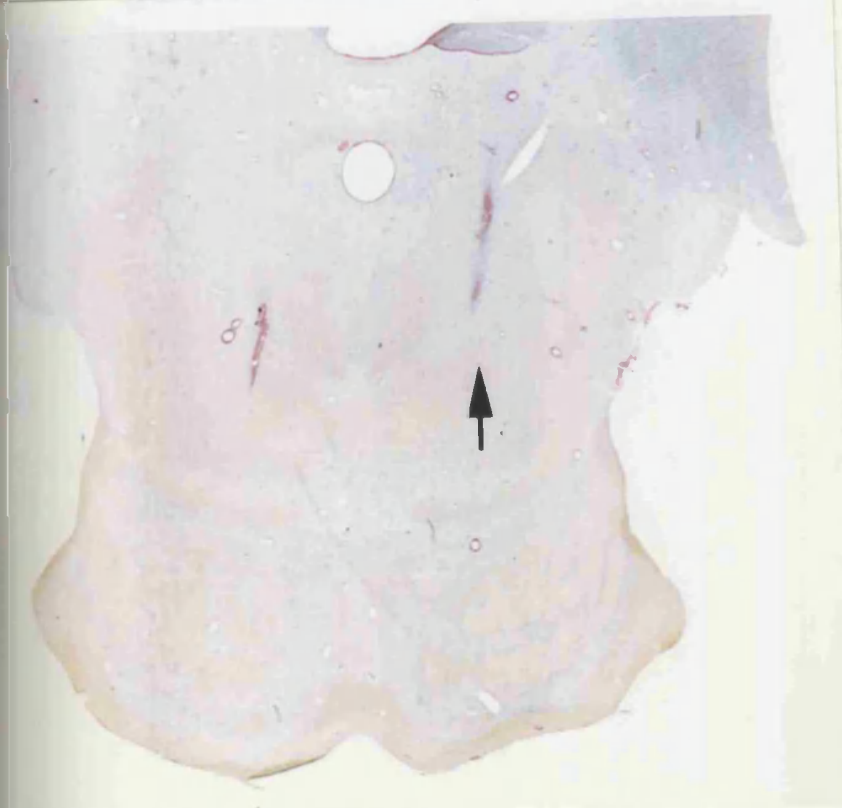

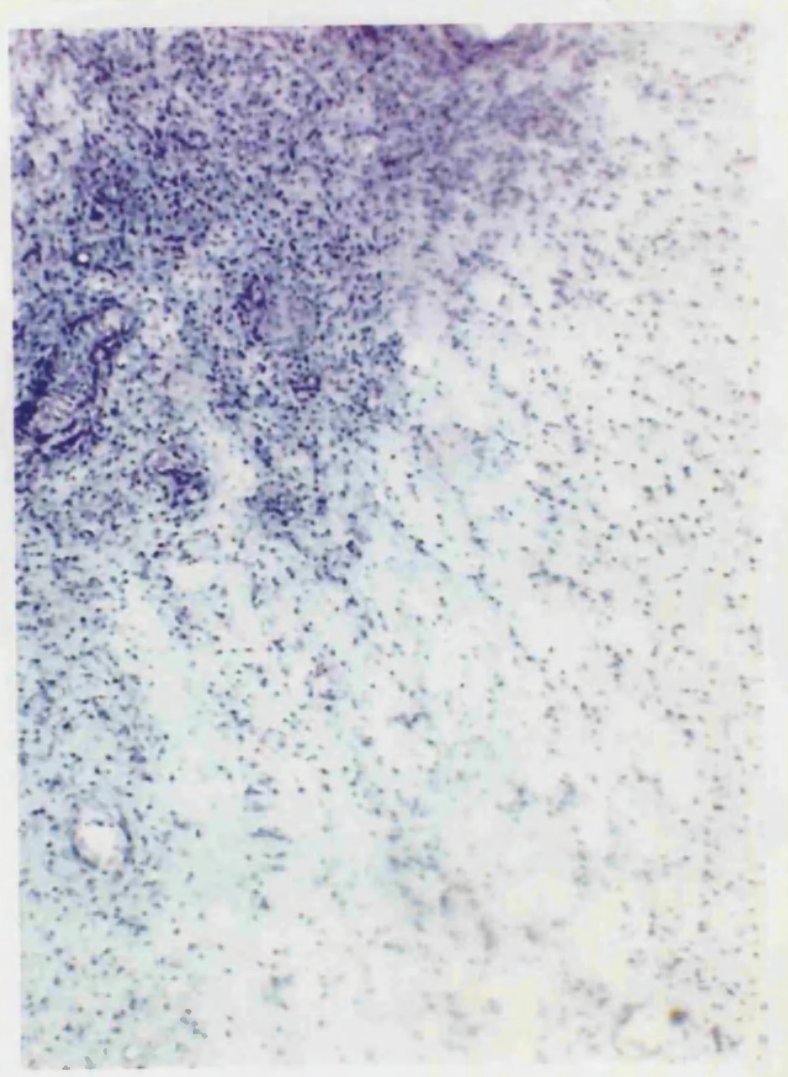


e.) Monkey E
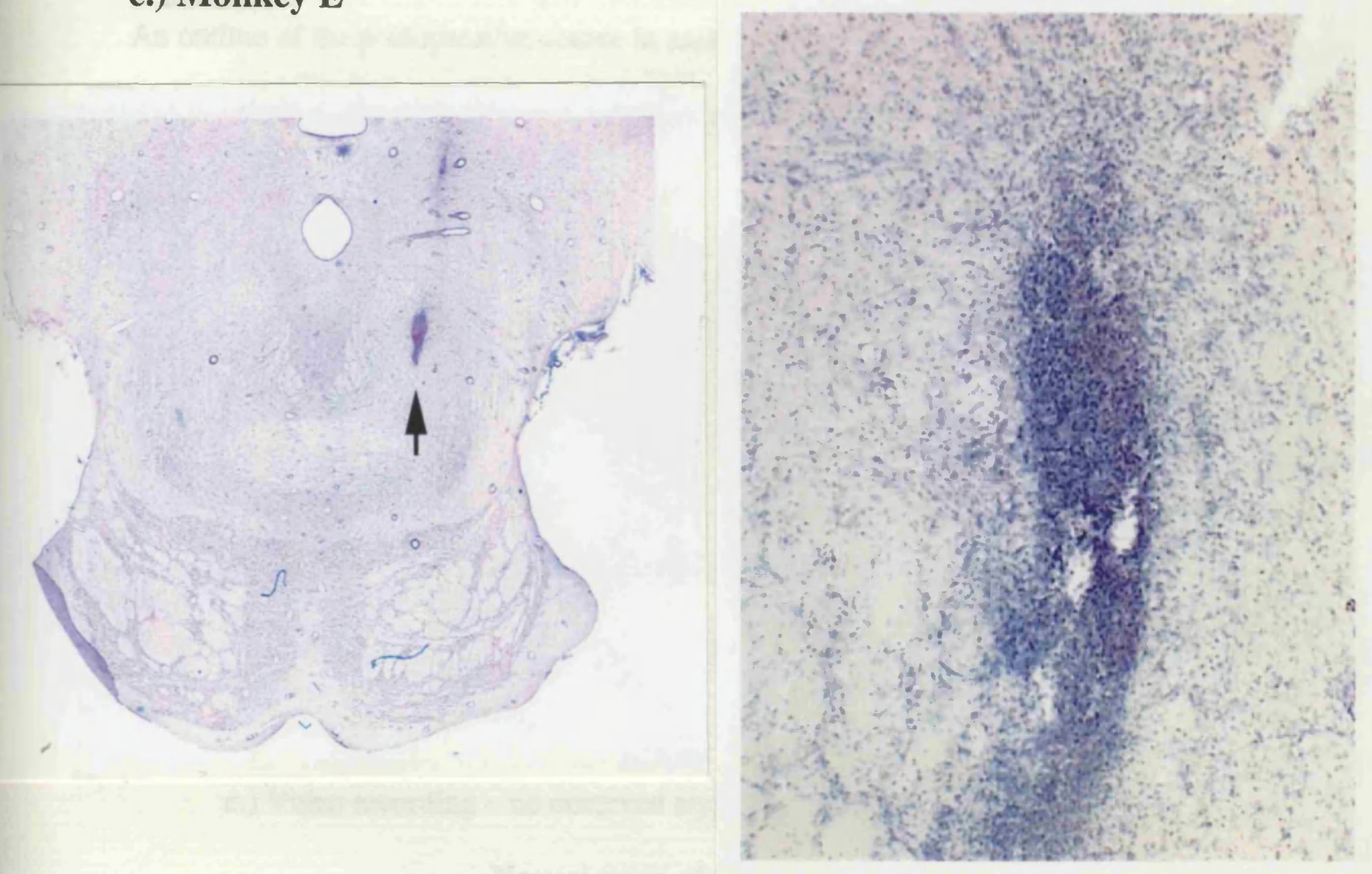


\section{RESULTS}

An outline of the postoperative course in each animal shall be presented

initially and then an overview of results discussed.

\section{Rhesus 1 (Monkey A in final analysis)}

Demographics : Female adult Rhesus

Age 16 years 11 months

Weight $7.1 \mathrm{~kg}$

Pre-operative Assessment:

a.)24hr activity counts $-2012 / 1921 / 2046 / 1617 / 1558 / 1967 / 1894$

Mean 24-hour activity count of $\mathbf{1 8 5 9}$ counts/day.

b.)Clarkes Score - Zero

c.) Video recording - no observed atypical behavior.

Normal range of facial expression.

Surgical Procedures:

a.)Day 0 - Right PPN lesion performed using $1 \mu$ of kainic acid

As described in materials and methods (Appendix 2).

b.)Day 8 - Left PPN lesion performed using $1 \mu l$ of kainic acid

As described in materials and methods (Appendix 2).

Post Operative Assessment:

a.)24hr activity counts $-0 / 120 / 1025 / 1224 / 1119 / 1322 / 1672$

Mean 24-hour activity count of 926 counts/day post unilateral lesion.

$0 / 143 / 120 / 323 / 319 / 211 / 814$

Mean 24-hour activity count of $\mathbf{2 7 6}$ counts/day post contralateral lesion.

b.)Clarkes Scoring -2 post unilateral lesion 
6 post contralateral lesion

c.)Video Recording - transient torticollis to left observed following unilateral lesion. Post bilateral lesions lack of facial expression, bradykinesia, some stooping and freezing. 


\section{Rhesus 2 (Monkey B in final analysis)}

Demographics : Female adult Rhesus

Age 6 years 3 months

Weight $8.4 \mathrm{~kg}$

Pre-operative Assessment:

a.) $24 h r$ activity counts -2589 / 2762 / 3849 / 3915 / $3389 / 3129 / 3747$

Mean 24-hour activity count of 3340counts/day.

b.)Clarkes Score - Zero

c.) Video recording - no observed atypical behavior.

Normal range of facial expression.

Surgical Procedures:

a.)Day 0 - Left PPN lesion performed using $1 \mu$ l of kainic acid

As described in materials and methods (Appendix 2).

b.)Day 8 - Right PPN lesion performed using $1 \mu \mathrm{l}$ of kainic acid

As described in materials and methods (Appendix 2).

Post Operative Assessment:

a.) $24 \mathrm{hr}$ activity counts $-3 / 386 / 1767 / 2016 / 1915 / 2269 / 2815$

Mean 24-hour activity count of $\mathbf{1 5 9 6}$ counts/day

post unilateral lesion.

$2 / 240 / 1361 / 1599 / 2342 / 2355 / 2487$

Mean 24-hour activity count of $\mathbf{1 4 8 4}$ counts/day

post contralateral lesion.

b.)Clarkes Scoring - 1 post unilateral lesion.

3 post bilateral lesions.

c.)Video Recording - mild hypokinesia and reduced facial expression

post bilateral lesions. 


\section{Rhesus 3(Monkey $\mathrm{C}$ in final analysis)}

Demographics : Female adult Rhesus

Age 6 years 10 months

Weight $4.9 \mathrm{~kg}$

Pre-operative Assessment:

a.) 24 hr activity counts -5788 / 3920 / 3977 / 3405 / 3999 / 4917 /3667

Mean 24-hour activity count of 4239counts/day.

b.)Clarkes Score - Zero

c.) Video recording - no observed atypical behavior.

Surgical Procedures:

a.)Day 0 - Left PPN lesion performed using $3 \mu$ l of kainic acid

As described in materials and methods (Appendix 2).

b.)Day 8 - Right PPN lesion performed using $3 \mu$ l of kainic acid

As described in materials and methods (Appendix 2).

Post Operative Assessment:

a.)24hr activity counts $-0 / 3 / 364 / 856 / 1087 / 1108 / 1996$

Mean 24-hour activity count of $\mathbf{7 7 3}$ counts/day post unilateral lesion.

$0 / 3 / 24 / 54 / 37 / 186 / 185$

Mean 24-hour activity count of 70counts/day post contralateral lesion.

b.)Clarkes Scoring -5 post unilateral lesion

10 post contralateral lesion

c.)Video Recording - severe hypokinesia, bradykinesia, freezing and stooping post-bilateral lesions. 


\section{Rhesus 4 (Not included in final analysis)}

Demographics : Female adult Rhesus

Age 6 years 9 months

Weight $7.3 \mathrm{~kg}$

Pre-operative Assessment:

a.) 24 hr activity counts $-3312 / 3402 / 3377$ / 2722 / $2690 / 2594 / 3114$

Mean 24-hour activity count of 3030 counts/day.

b.)Clarkes Score - Zero

c.) Video recording - no observed atypical behavior.

Normal range of facial expression.

Surgical Procedures:

a.)Day 0 - Simultaneous bilateral PPN lesions

Using $1 \mu$ of kainic acid each side.

As described in materials and methods (Appendix 2).

Post Operative Assessment:

a.) $24 \mathrm{hr}$ activity counts $-0 / 0$

Aspiration and death at 2 days post operative. 


\section{Cyno 1(Monkey D in final analysis)}

Demographics : Male adolescent Cynomologus

Age 4 years 10 months

Weight $3.2 \mathrm{~kg}$

Pre-operative Assessment:

a.)24hr activity counts $-1619 / 1742$ / 2208 / 2885 / 2559 / $2604 / 2584$

Mean 24-hour activity count of $\mathbf{2 3 1 4}$ counts/day.

b.)Clarkes Score - Zero

c.) Video recording - no observed atypical behavior.

Surgical Procedures:

a.)Day 0 - Bilateral PPN lesions performed

Using $1 \mu l$ of kainic acid each side

As described in materials and methods (Appendix 2).

Post Operative Assessment:

a.)24hr activity counts $-0 / 8 / 86 / 205 / 222 / 544 / 300$

Mean 24-hour activity count of 191 counts/day post bilateral lesions.

b.)Clarkes Scoring - 2 post bilateral lesions

c.)Video Recording - moderate hypokinesia and bradykinesia post bilateral lesions, with reduced facial expression. 


\section{Cyno 2 (Monkey $E$ in final analysis)}

Demographics : Male adult Cynomologus

Age 5 years 6 months

Weight $5.5 \mathrm{~kg}$

Pre-operative Assessment:

a.) $24 \mathrm{hr}$ activity counts $-817 / 921 / 1003 / 1087 / 1057 / 1121 / 1007$

Mean 24hour-activity count of $\mathbf{1 0 0 1}$ counts/day.

b.)Clarkes Score - Zero

c.) Video recording - no observed atypical behavior.

Surgical Procedures:

a.)Day 0 - Bilateral PPN lesions performed

Using1 $\mu$ l of kainic acid each side

As described in materials and methods (Appendix 2).

Post Operative Assessment:

a.)24hr activity counts $-0 / 3 / 24 / 54 / 37 / 186 / 185$

Mean 24-hour activity count of 70counts/day post bilateral lesions.

b.)Clarkes Scoring - 5 post bilateral lesions

c.)Video Recording - severe hypokinesia and moderate bradykinesia post bilateral lesions, with reduced facial expression. 


\section{Cyno 3(Not included in final analysis)}

Demographics : Male adolescent Cynomologus

Age 4 years 2 months

Weight $2.7 \mathrm{~kg}$

Pre-operative Assessment:

a.)24hr activity counts $-800 / 618 / 898 / 1021 / 1201 / 1252 / 1066$

Mean 24hour-activity count of 979 counts/day.

b.)Clarkes Score - Zero

c.) Video recording - no observed atypical behavior.

Surgical Procedures:

a.)Day 0 - Left PPN lesion performed using $1 \mu$ l kainic acid

As described in materials and methods (Appendix 2).

Post Operative Assessment:

a.) $24 \mathrm{hr}$ activity counts $-0 / 0 / 0 / 0 / 0$

Sacrificed at 6 days post operative due to condition - severe Right hemiparesis with facial weakness and Bulbar dysfunction.

Histology revealed extensive hemorrhage secondary to ventriculogram access site. 


\section{Summary of Results}

Complete recovery from althesin anaesthesia in the primate takes one to two hours. However after both the unilateral and bilateral lesions the animals were completely akinetic for at least 24 hours following surgery. Nevertheless, all animals were 'alert' within an hour post operative, i.e. their eyes were open with conjugate gaze and visual tracking, but they made minimal spontaneous movements and exhibited increased tone in all limbs. Nevertheless withdrawal from a noxious stimulus remained brisk during this period.

Complications arising in two cases result in complete data being available for three staged and two simultaneous PPN lesioning procedures only. In the animal receiving a single unilateral PPN lesion a dense, unresolving right hemiparesis with hypertonia and hyperreflexia was observed post operatively necessitating euthanasia at 6 days post operatively. Histology in this case revealed a well sited lesion of the left PPN with no associated abnormalities. However, a hemorrhage into the left corpus callosum and adjacent motor cortex was evident and appeared to be secondary to the needle tract utilized for the operative ventriculogram. In a second instance aspiration and death occurred at 2 days post operative in a densely akinetic animal following simultaneous bilateral lesions. Unfortunately histology was not possible in this instance to confirm lesion siting. No data from either of these cases has been included in our collective analysis. Due to the variability in the baseline daily activity between individual monkeys and the small numbers available for our study, results have been analyzed individually. 
After unilateral PPN lesions all three monkeys displayed a profound reduction in spontaneous activity over the initial 48-hours post operatively (Table $4.2 \&$ Fig 4.4). In the subsequent 5 days a recovery of spontaneous activity was seen, although 7 days post operatively that spontaneous activity remained less than that recorded pre operatively in all cases. In monkeys $\mathrm{A}$ and $\mathrm{B}$, where the volume of $0.1 \%$ kainic acid used to create a lesion was 1 microlitre, activity levels were reduced by 10 and $16 \%$ respectively by 7 days post operatively. Comparing the animals' activity averaged over the 7 days after surgery with that before, the reduction was highly statistically significant in both animals $(t$ $=3.8 \& 4.0, \mathrm{p}<0.001 \& \mathrm{p}<0.001$ respectively). In the case of monkey $\mathrm{C}$, where the volume of $0.1 \%$ kainic acid used to create a lesion was 3microlitres, the reduction in recorded activity remained reduced by $53 \%$ at 7 days post operative. This too represented a highly significant reduction in activity over the week following surgery $(t=8.4, p<0.000)$. At all times the monkeys movements remained well co-ordinated, though bradykinetic as compared to their speed of movement pre operatively, with no signs of cerebellar ataxia nor intention tremor. In contrast reflex movements, such as withdrawal from a noxious stimulus, remained brisk and seemingly unaffected. On assessment on the Clarke Parkinsonism scale for primates all animals displayed an increase in their scores from their pre operatively 'normal' scores of zero (Table 4.5). After a further contralateral lesion was performed in monkeys A, B \& C there was a further decrease in the level of spontaneous activity as recorded by the Doppler meter (Table 4.3 \& Fig 4.5). Once again this decrease was most marked in monkey $\mathrm{C}(99.5 \%$ reduction in activity at 7 days postoperative) in which a 3 microlitre volume of kainate was injected. As was the case following 
unilateral lesions co-ordination of movements and speed of withdrawal reflexes remained intact. Also no clinical evidence of cerebellar dysfunction was observed in these animals at this time. A further increase in their Clarke's rating was seen in all three monkeys (Table 4.5).

In monkeys $\mathrm{D} \& \mathrm{E}$ simultaneous bilateral lesions were performed using 1 microlitre of $0.1 \%$ kainic acid to form each lesion. In both cases a marked reduction in spontaneous activity was observed which persisted at an extremely high level of impairment over the course of the postoperative week. Monkey D showed an $87 \%$ reduction in spontaneous activity at 7 days post operatively, whilst in Monkey E activity was reduced by $91 \%$ at the same time post operatively (Table $4.4 \&$ Fig 4.5 ). These results are highly statistically significant also $(t=10.9 \& 18.7, p<0.000$ respectively). Once again these animals exhibited no inco-ordination, tremor nor lack of arousal and they also showed an increase in the Clarke rating (Table 4.5).

A further observation made post operatively, and confirmed on review of the videotapes of all animals, was a reduction in facial expressiveness following bilateral lesions, with an absence of 'threat' behavior such as teeth baring with head propulsion as seen frequently in the normal primate.

Histology was performed on Monkeys A,B,C,D \& E as described above. In all five cases, areas of focal necrosis were revealed at the distal end of the fine needle tracts. Most included a portion of the PPN region; however, some were better sited than others and the degree of gliosis seen also varied ( Fig 4.3a-e). In Monkey B the initial left sided lesion is seen to lie dorsal to the PPN region, whereas the subsequent right sided lesion is better placed to include part of the targeted region, but only a small degree of gliosis is demonstrated at the distal 
end of the needle tract (Fig 4.3b). This animal recovered best following both procedures, and only suffered a $26 \%$ reduction in activity following bilateral lesioning. This may therefore be a consequence of a small well sited unilateral lesion (right) alone, as the left sided lesion cannot be said to have involved the PPN region. In Monkey $\mathrm{C}$, where the reduction in activity was most marked and the volume of kainate used was 3 microlitres, histology shows a large area of gliosis centred on the PPN region bilaterally (Fig 4.3c demonstrates the left lesion). In the remaining three animals, the lesions demonstrated almost certainly represent the ablation of an area including part PPN region having a volume of approximately $1-3 \mathrm{~mm}^{3}$, the larger volume lesion being associated with the larger injection volume in Monkey C. The Red Nucleus, surrounding mesencephalic locomotor region and fibers of the superior cerebellar peduncle remained intact in all cases. 
Table 4.2. 24 Hour Activity Counts Following Unilateral Cytotoxic PPN Lesions in Monkeys A, B \& C.

\begin{tabular}{|c|c|c|c|c|c|c|c|c|c|c|}
\hline & $\begin{array}{l}\text { Volume of } \\
\text { Lesioning } \\
\text { Kainate }\end{array}$ & $\begin{array}{l}\text { Mean } \\
24 \text { hour } \\
\text { Activity } \\
\text { Count }\end{array}$ & $\begin{array}{l}\text { Mean } \\
24 \text { hour } \\
\text { activity } \\
\text { count }\end{array}$ & \multicolumn{7}{|c|}{ Post Operative Days } \\
\hline & & & & 1 & 2 & 3 & 4 & 5 & 6 & 7 \\
\hline $\begin{array}{l}\text { Monkey } \\
\text { A } \\
\end{array}$ & 1 microl & $\begin{array}{l}1859 \\
( \pm 73) \\
\end{array}$ & $\begin{array}{l}926^{*} \\
( \pm 237) \\
\end{array}$ & 0 & 120 & 1025 & 1224 & 1119 & 1322 & 1672 \\
\hline $\begin{array}{l}\% \\
\text { Reduction }\end{array}$ & & & & 100 & 93 & 45 & 34 & 29 & 29 & 10 \\
\hline $\begin{array}{l}\text { Monkey } \\
\text { B }\end{array}$ & 1 microl & $\begin{array}{l}3340 \\
( \pm 201)\end{array}$ & $\begin{array}{l}1596 * \\
( \pm 386)\end{array}$ & 3 & 386 & 1767 & 2016 & 1915 & 2269 & 2815 \\
\hline $\begin{array}{l}\% \\
\text { Reduction }\end{array}$ & & & & 100 & 88 & 47 & 40 & 43 & 32 & 16 \\
\hline $\begin{array}{l}\text { Monkey } \\
\text { C }\end{array}$ & 3microl & $\begin{array}{l}4239 \\
( \pm 313)\end{array}$ & $\begin{array}{l}773 * \\
( \pm 270)\end{array}$ & 0 & 3 & 364 & 856 & 1087 & 1108 & 1996 \\
\hline $\begin{array}{l}\% \\
\text { Reduction }\end{array}$ & & & & 100 & 100 & 91 & 80 & 74 & 74 & 53 \\
\hline & & & & & & & & & & \\
\hline
\end{tabular}

$* \mathrm{t}=3.8-8.4, \mathrm{p}<0.007-0.000$ on $\mathrm{t}$-testing. (Comparing 7 days pre op with 7 days post op) 
Table 4.3. 24 Hour Activity Counts Following Contralateral

Cytotoxic PPN Lesions in Monkeys A, B \& C.

\begin{tabular}{|c|c|c|c|c|c|c|c|c|c|c|}
\hline & $\begin{array}{l}\text { Volume } \\
\text { of } \\
\text { Lesioning } \\
\text { Kainate }\end{array}$ & $\begin{array}{l}\text { Mean } \\
24 \text { hour } \\
\text { activity }\end{array}$ & $\begin{array}{l}\text { Mean } \\
24 \text { hour } \\
\text { activity }\end{array}$ & \multicolumn{7}{|c|}{ Post Operative Days } \\
\hline & & & & 1 & 2 & 3 & 4 & 5 & 6 & 7 \\
\hline $\begin{array}{l}\text { Monkey } \\
\text { A }\end{array}$ & 1microl & 1859 & $\begin{array}{l}276^{*} \\
( \pm 100)\end{array}$ & 0 & 143 & 120 & 323 & 319 & 211 & 814 \\
\hline $\begin{array}{l}\% \\
\text { Reduction }\end{array}$ & & & & 100 & 92 & 93 & 83 & 83 & 89 & 56 \\
\hline Monkey B & 1microl & 3340 & $\begin{array}{l}1484^{*} \\
( \pm 389)\end{array}$ & 2 & 240 & 1361 & 1599 & 2342 & 2355 & 2487 \\
\hline $\begin{array}{l}\% \\
\text { Reduction }\end{array}$ & & & & 100 & 93 & 59 & 52 & 30 & 29 & 26 \\
\hline $\begin{array}{l}\text { Monkey } \\
\text { C }\end{array}$ & 3microl & 4239 & $\begin{array}{l}13 * \\
( \pm 3)\end{array}$ & 0 & 3 & 10 & 24 & 18 & 16 & 21 \\
\hline \begin{tabular}{|l|}
$\%$ \\
Reduction \\
\end{tabular} & & & & 100 & 100 & 100 & 99.5 & 99.5 & 99.5 & 99.5 \\
\hline & & & & & & & & & & \\
\hline
\end{tabular}

$* t=4.2-13.6, \mathrm{p}<0.001-0.000$ on $\mathrm{t}-$ testing.

(Comparing 7 days pre op with 7 days post op) 
Table 4.4. 24 Hour Activity Counts Following Simultaneous

Bilateral Cytotoxic PPN Lesions in monkeys D \& E.

\begin{tabular}{|c|c|c|c|c|c|c|c|c|c|c|}
\hline & $\begin{array}{l}\text { Volume } \\
\text { of } \\
\text { Lesioning } \\
\text { Kainate }\end{array}$ & $\begin{array}{l}\text { Mean } \\
24 \text { hour } \\
\text { activity } \\
\text { count }\end{array}$ & $\begin{array}{l}\text { Mean } \\
24 \text { hour } \\
\text { activity } \\
\text { count }\end{array}$ & Post & Opera & ve D & & & & \\
\hline & & & & 1 & 2 & 3 & 4 & 5 & 6 & 7 \\
\hline $\begin{array}{l}\text { Monkey } \\
\text { D }\end{array}$ & $1 \mathrm{microl}$ & 2314 & $\begin{array}{l}191^{*} \\
( \pm 72)\end{array}$ & 0 & 8 & 86 & 205 & 222 & 544 & 300 \\
\hline $\begin{array}{l}\% \\
\text { Reduction }\end{array}$ & & & & 100 & 100 & 96 & 91 & 90 & 76 & 87 \\
\hline Monkey E & $1 \mathrm{microl}$ & 1001 & $\begin{array}{l}70^{*} \\
( \pm 31)\end{array}$ & 0 & 3 & 24 & 54 & 37 & 186 & 185 \\
\hline $\begin{array}{l}\% \\
\text { Reduction }\end{array}$ & & & & 100 & 100 & 98 & 95 & 96 & 91 & 91 \\
\hline & & & & & & & & & & \\
\hline
\end{tabular}

$*_{\mathrm{t}}=10.9-18.7, \mathrm{p}<0.000$ on $\mathrm{t}$-testing.

(Comparing 7 days pre op with 7 days post op) 
Table 4.5. Clarke Rating Scores Pre and Post Operative.

\begin{tabular}{llll}
\hline & Pre Operative & $\begin{array}{l}\text { Post Unilateral } \\
\text { Lesion }\end{array}$ & $\begin{array}{l}\text { Post Bilateral } \\
\text { Lesion }\end{array}$ \\
\hline Monkey A & 0 & 2 & 6 \\
Monkey B & 0 & 1 & 3 \\
Monkey C & 0 & 5 & 10 \\
Monkey D & 0 & & 2 \\
Monkey E & 0 & & 5 \\
\hline
\end{tabular}


Figure 4.4 . Individual 24 hour Activity Counts in Monkeys A - C Following Unilateral Cytotoxic Lesions of the PPN.
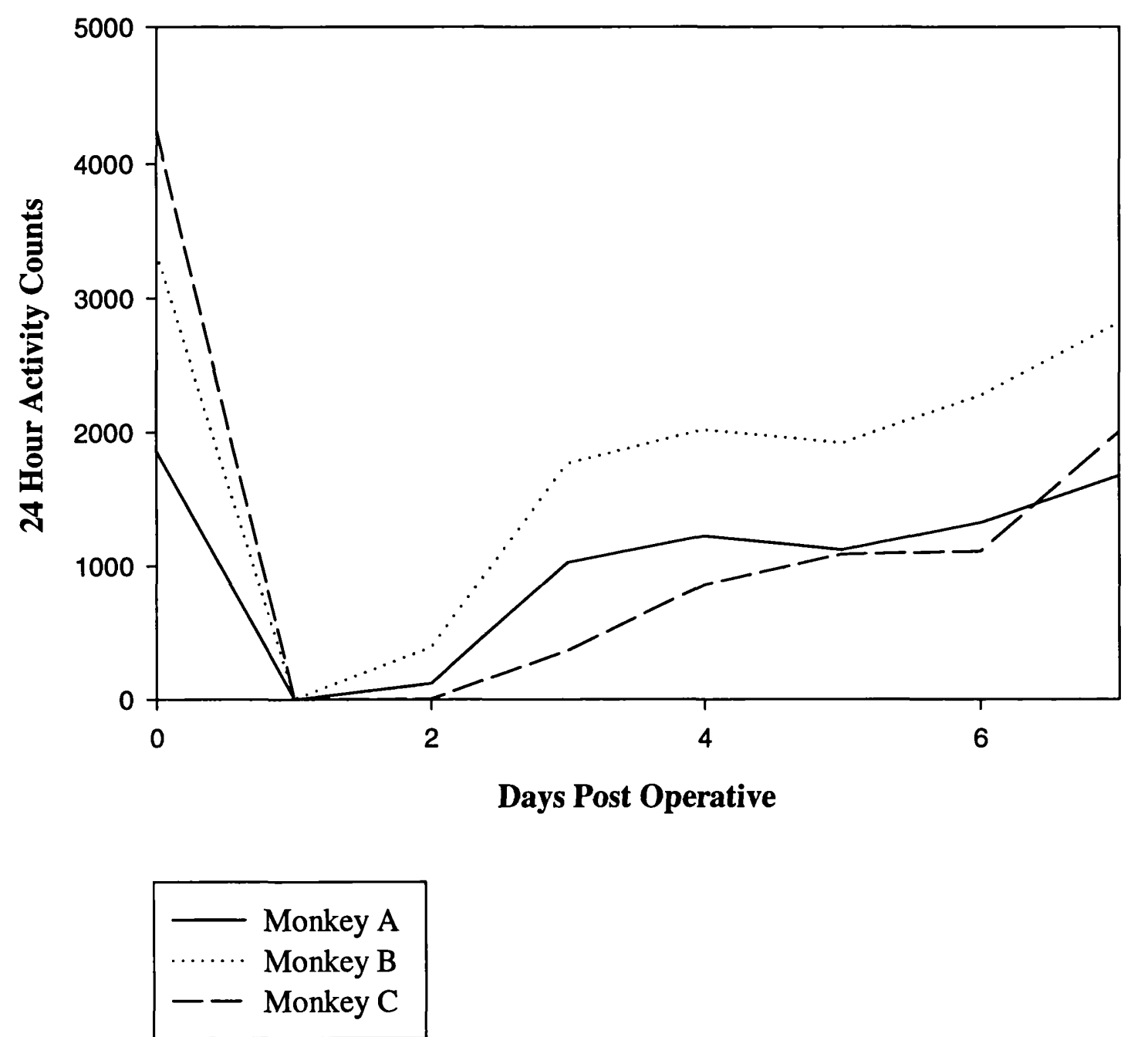
Figure 4.5 . Individual 24 Hour Activity Counts in Monkeys A - E Following Bilateral Cytotoxic Lesions of the PPN.

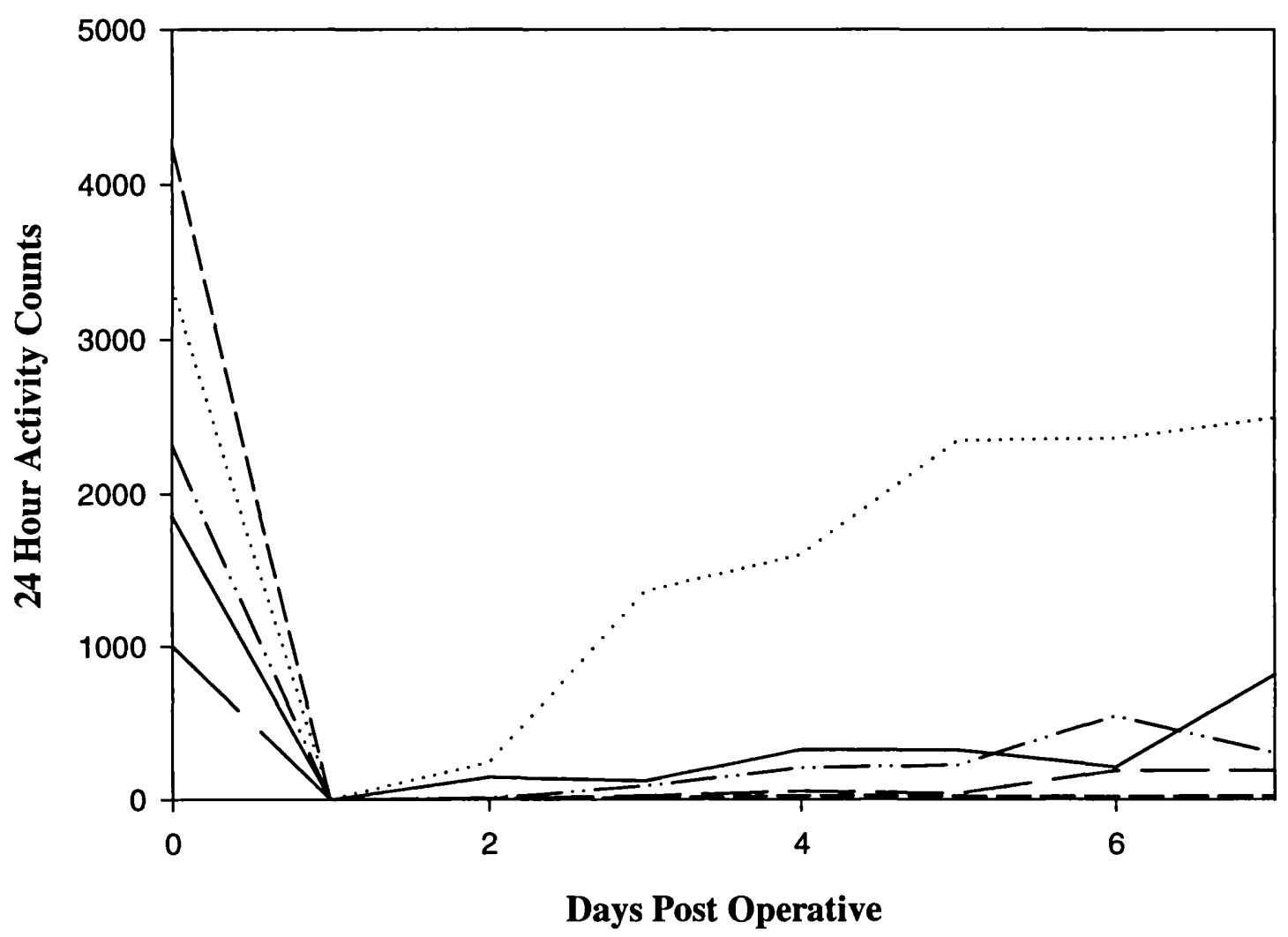

- Monkey A Monkey B

- Monkey C

-... Monkey D

- Monkey E 


\title{
CHAPTER FIVE
}

\author{
DISCUSSION
}




\section{DISCUSSION}

Interest in the role of the PPN in the circuitry of movement control, and hence in akinetic states, has been fuelled by a number of findings over recent years;

(i) The PPN receives dense projections from GPm and in turn projects to the pontine and medullary reticulospinal systems (Jackson et al 1983), in addition to its abundant ascending projections.

(ii) In rats PPN stimulation produces locomotor activity, whilst inactivation by GABA agonists decreases activity (Brudzynski et al 1986, Garcia-Rill 1987, Morgenson et al 1988, Milner et al 1988).

(iii) There is degeneration of the PPN in Parkinson's Disease and in the akinetic syndrome of Supranuclear Palsy (Zweig et al 1985, Hirsch et al 1987, Jellinger et al 1988, Zweig et al 1989, Malessa et al 1991).

(iv) The administration of MPTP in primates has been shown to result in the accumulation of radiolabelled 2-deoxyglucose in the PPN, possibly as a result of increased pallidal outflow in the absence of dopamine inhibition of the striatum (Mitchell et al 1989).

(v) Unilateral excitotoxic lesions of the PPN produce contralateral bradykinesia (Kojima et al 1997).

These findings led us to perform bilateral radiofrequency lesions of the PPN in order to ascertain whether indeed suppression of activity within this region is associated with a decrease in motor activity. Having done so we progressed to 
perform excitotoxic lesions of this area to confirm that selectively destroying neurons there, rather than axons of passage, causes this akinesia.

It is clear that our lesions resulted in a reduction in both the speed and quantity of the animal's spontaneous movements in both instances.

In the case of radiofrequency lesioned animals, after unilateral lesions the quantity and speed of the monkeys' movements reduced to approximately one third of their pre-operative level and their facial expressions became fixed. The reduction in their activity was highly statistically significant. However, they recovered to $55 \%$ of control values on the second day and returned to normal over the next week. After the second such lesion in the first three monkeys, or bilateral lesions in the subsequent three monkeys, the poverty and slowing of the animals movements showed little improvement in the week following surgery. On the first post operative day activity counts had dropped by $74 \%$, and on the seventh day they were still reduced by over $70 \%$. Also the monkeys' lack of facial expression persisted, a fact confirmed on reviewing the videotapes.

In animals undergoing cytotoxic lesions where the volume of kainate used was 1 microlitre, those undergoing unilateral lesions recovered to within 10$16 \%$ of normal activity over the course of 7 days. Even with a larger 3 microlitre unilateral lesion recovery occurred to almost $50 \%$ of normal within one week. This recovery is most likely due to the ascending and descending projections from each PPN to the basal ganglia and pontomedullary reticulospinal systems being bilateral. Hence the intact side could take over during the recovery period. In the case of bilateral lesions the animals' activity remained significantly depressed at the end of one week post operative, with activity levels remaining most markedly reduced following simultaneous, 
smaller lesions or staged larger lesions. The recovery after bilateral lesions, though prolonged, may well have resulted from the fact that the lesions were extremely small even in relation to the structure targeted, thus allowing undamaged neurons to take over on both sides after a sufficient recovery period. This hypothesis is supported by our finding that the most marked effect of all was seen subsequent to staged larger lesions, and that permanent contralateral effects have been demonstrated in unilaterally lesioned animals where the lesions made were five times greater than those in our series (Kojima et al 1997). The reductions in activity we have demonstrated would therefore support the hypothesis that Parkinsonian akinesia results from over inhibition of the PPN region by an overactive GPm.

Dyskinesias are alleviated bilaterally by a medial pallidotomy (though the ipsilateral effect is less dramatic) but only tremor is alleviated by thalamotomy. These results suggest that dyskinesias are a pallido-brainstem phenomenon. Lesioning the medial pallidum also alleviates the bradykinesia/akinesia of parkinsonism. It is interesting that bilateral pallidotomies are more effective than unilateral lesions in this respect (Johannson et al 1997, Scott et al 1998). This would suggest that although the ascending and descending projections of the PPN are bilateral, both need to be released from pallidal inhibition for full movement to be restored.

The PPN is thought to be an important interface nucleus between sensory feedback inputs, arousal and motivational systems to perform motor tasks and the actual production of stereotyped movements such as locomotion. Although little is known about the anatomical and pharmacological connections of the human PPN, animal studies have shown strong links between the PPN and the basal ganglia. PPNc neurons provide cholinergic inputs to the thalamus and $\mathrm{SNc}$ and receive important sensory feedback information from the spinal cord. 
Glutamatergic PPNd neurons receive the main STN, GPm and SNr outputs and provide the main outflow of information to the lower brainstem and spinal cord. Electrophysiological studies have suggested that cholinergic PPNc neurons tend to fire tonically with a 'non-bursting' pattern and may be important for modulation of steady-state locomotion, while glutamatergic PPNd neurons tend to fire in bursts and may be important in providing the signal to 'call-up' existing and preserved locomotory motor programs for gait initiation. 'Non-bursting' cholinergic PPNc neurons provide inputs to the thalamus and $\mathrm{SNr}$ that may be important for co-ordinating sensory feedback information and for setting a level of arousal and motivation to perform locomotor tasks and maintain steady-state locomotion. In PD, PPN neurons degenerate and alterations in the balance of excitatory and inhibitory inputs to the PPN from the STN, GPm and SNr may be responsible for akinesias and gait disturbances that are particularly refractory to drug treatment. Bilateral STN lesioning and deep brain stimulation may produce their therapeutic effects by normalizing some of the altered signals to the PPN from STN, GPm and SNr.

It could be argued that the reduction in the animals' activity which we witnessed was the result of non-specific effects on arousal. However had this been the case then we would have expected the monkeys to have slept for large periods of the day during which they were inactive. This was never the case, and video recordings showed all animals to have unaltered sleep/wake cycles from preoperatively. They simply moved less often and more slowly during their awake period following surgery.

We cannot be certain the degree to which the effects of our lesions were due to damage of only the cholinergic population of the PPN, as all almost certainly included not only this cell sub-population but also glutamatergic cells of PPNd 
and neurones in the surrounding region. The cholinergic PPNc neurons are also involved in setting levels of motivation and arousal. Since these remained unchanged, as far as we could determine, probably a significant proportion of the cholinergic neurones remained intact. The glutamatergic PPNd neurons are 'bursters' which are probably recruited for activity during movement. Thus damage of these cells, as was almost certainly present, could explain the clinical manifestations of gait ignition failure and akinesia. It may well be that the effects which we observed are compound and the result of damage to a combination of the cell populations rather than group specific. Thus the term pedunculopontine region rather than pedunculopontine nucleus more accurately reflects the area that mediated our observed effects.

Questions arising subsequent to this work include; is this a dopa responsive akinesia, and could the effects be replicated by the selective destruction of either of the sub-populations of neurons within the PPN region? If our hypothesis is correct; i.e. that parkisonian akinesia is the result of excessive inhibition of the PPN region by overactive descending pallidal outputs leading to the depletion of active neurones in this area, then we would not expect the administration of dopamine or a dopamine agonist to alter the akinesia generated by PPN lesions. Although dopamine acts to re-regulate the balance within the direct and indirect pathways thus reducing GPm output to more normal levels in the parkinsonian state, it plays no part in the afferent and efferent connections of the PPN region. Thus in the presence of a depleted neuronal population in the PPN region, as caused by our lesioning and possibly in the later stages of Parkinson's Disease, dopamine supplementation is unable to reactivate these damaged pathways, as would be necessary to restore locomotion control. 
The question whether selective destruction of the cholinergic or glutamatergic cell groups within the PPN would result in akinesia is important in the implications which it bears on possible clinical therapies. Should it be that a depletion in either of these neuronal populations is solely responsible for the generation of akinesia, then the use of a selective agonist (cholinergic or glutamatergic) may prove an effective therapy in such conditions (PD, PSP, gait ignition failure).

Further possible therapies in such akinetic syndromes which might be possible should our proposed role for the PPN region in locomotion control be correct include the use of stimulators or cellular transplantation into this area. As we have discussed earlier the use of deep brain stimulators in the treatment of movement disorders is now well established with the current implantation sites being; thalamus (for tremor), medial pallidum (for dyskinesias, bradykinesias, akinesia) and more recently the STN (for all parkinsonian symptoms particularly those involving axial muscles). If indeed the reduction in output from a chronically overinhibited PPN is central to the generation of akinesia then it should be feasible to reverse these effects by stimulation of the region. Implantation would be no more technically difficult and surgery no more hazardous than for current sites. As regards the option of cellular transplantation into this region, again the question of the exact role played by the cholinergic and glutamatergic populations arises. However, the possibility of replacing either or both cell lines by this method may provide an effective therapy in the future.

Further elucidation of the mechanisms underlying akinesia has clinical implications beyond the therapy of Parkinson's Disease alone, as does a fuller understanding of the widely ramifying efferent connections of the PPN. Also our proposed role for the PPN in movement control would provide a possible 
explanation for the variation seen in clinical outcome following pallidotomy in Parkinsonian patients. The patients who demonstrate a lesser improvement in their symptoms (bradykinesia, dyskinesia, tremor) after medial pallidotomy may have a more severe degree of degeneration of the PPN, and therefore less ability to recover following the release from over inhibition of this structure which is afforded by medial pallidotomy. Should it be shown definitively that these brainstem mechanism are central to the generation of akinesia, then the therapeutic possibilities of pharmacological or electrical stimulation, or cellular implantation in the PPN region as discussed above should be considered further. 


\section{CONCLUSIONS}




\section{CONCLUSIONS}

The main conclusion of this work is that destruction of neurons within the region of the PPN result in a parkinsonian like akinesia. It may therefore be, that the clinical symptom of akinesia as seen in PD patients results from excessive inhibition of the PPN region by overactive inhibitory GPm outputs.

Further clarification of these matters serves an important clinical purpose. More effective therapies for such states could be evolved utilizing pharmacological agonists targeted at the PPN, stimulation or implantation in this region alone, or in conjunction with current areas targeted. 


\section{REFERENCES}




\section{REFERENCES}

Agid Y, Javoy-Agid F, et al. Ed. (1987). Biochemistry of neurotransmitters in Parkinson's disease. Movement Disorders. London, Butterworths.

Alexander G and Crutcher M (1990). Functional architecture of basal ganglia circuits: neural substrates of parallel processing. TINS 13(7): 266-271.

Andy O J, Jarko M F, et al. (1963). Subthalamotomy in treatment of parkinsonian tremor. J. Neurosurg. 20: 860-870.

Aziz T and Torrens M (1989). CT-guided thalamotomy in the treatment of movement disorders. B.J.Neurosurg; 3: 333-336.

Aziz T, Peggs D, Sambrook et al (1991). Lesions of the subthalamic nucleus for the alleviation of MPTP induced parkinsonism in the primate. Mov Disord; 6:288-293.

Aziz TZ, Peggs D, Agarwal E, et al (1992). Subthalamic nucleotomy alleviates parkinsonism in the MPTP exposed primate. Br J Neurosurg; 6:575582.

Aziz TZ. (1992). Lesions of the subthalamic nucleus alleviate parkinsonism in the MPTP treated primate. MD Thesis. University of Manchester.

Barbeau A, Roy M, et al. (1986). Environmental and genetic factors in the etiology of Parkinson's disease. Advances in Neurology. New York, Raven Press. 299-306.

Baron, J. (1986). Cigarette smoking and Parkinson's disease. Neurology; 36: 1490-1496. 
Baron M, Vitek J, Bakay R, et al (1996). Treatment of advanced Parkinson's

Disease by posterior Gpi pallidotomy: 1 year results of a pilot study. Ann Neurol; 40:355-366.

Beaulieu M (1987). Clinical importance of D-1 and D-2 receptors. Can J Neurol Sci; 14: 402-406.

Bejjani B, Damier R, Arnulf I, et al (1997). Pallidal stimulation for Parkinson's Disease. Two targets ? Neurology; 79:1564-1569.

Benabid A, Pollak P, et al (1989). Chronic VIM-thalamic stimulation in movement disoeders. Neural mechanisms in disorders of movement. London, Paris, John Libbey. 413-415.

Benabid A, Pollack P, Dongming, et al (1996). Chronic electrical stimulation of the ventralis intermedius nucleus of the thalamus as a treatment of a movement disorder. Neurosurg; 84:203-214.

Benazzouz A, Gross C, Feger J, et al (1993). Reversal of rigidity and improvement in motor performance of subthalamic high frequency stimulation in MPYP-treated monkeys. Eur J Neurosci; 5:382-389.

Benazzouz A, Beraud T, Feger J, et al (1996). Alleviation of experimental hemiparkinsonism by high frequency stimulation of the subthalamic nucleus in primates. A comparison with L-dopa treatment. Mov Disord; 11(6):627632. 
Bergman H, Wichmann T, DeLong M (1990). Reversal of experimental parkinsonism by lesions of the subthalamic nucleus. Science; 249:1436-1438.

Bertolucci P, Andrade L, et al (1987). Total sleep deprivation and Parkinson's disease. Arq Neuropsiquitr; 45: 224-230.

Bevan M, Bolam P (1995). Cholinergic, GABAergic and glutamate enriched inputs from the mesopontine tegmentum to the subthalamic nucleus in the rat. Neuroscience; 15:715-720.

Birkmayer W, Knoll J, et al (1985). Increased life expectancy resulting from addition of L- Deprenyl to Madopar treatment in Parkinson's disease. J Neural Transm; 64: 113-127.

Blaha CD, Winn P (1993). Modulations of dopamine efflux in the striatum following cholinergic stimulation of the substantia nigra in intact and pedunculopontine tegmental nucleus lesioned rats. J Neurosci; 13:1035-1044.

Blocq P and Marinesco G (1893). Sur un cas de tremblement parkinsonien hemiplegique symptomatique d'une tumeur du pedoncle cerebral. Comptes rendus de la Societe de Biologie_Paris; 5: 105-111.

Bonfati V, Fabrizio E, Vanacore, et al (1995). Familial Parkinson's disease: a clinical and genetic analysis. Can J Neurol Sci; 22:272-279.

Boraud T, Bezard E, Biculac B, et al (1996). High frequency stimulation of the internal globus pallidus (Gpi) simultaneously improves parkinsonian symptoms and reduces the firing frequency of Gpi neurons in the MPTP treated monkey. Neurosci Lett; 215:17-20. 
Brewis M, Rolland C, Miller H (1966). Neurological disease in an English city. Acta Neurol Scan; 42(Suppl.24): 1-89.

Brissaud E (1894). Lecon sur les maladies nerveuses. Nature et pathogenie de la maladie de Parkinson. J Medic Chirurg Pract : 488-501.

Browder J (1948). Section of fibres of the anterior limb of the internal capsule in parkinsonism. Am. J Surg; 75: 264-268.

Brudzynski SM, Houghton PE, et al (1986). Involvement of neuronal cell bodies in the mesencephalic locomotor region in the initiation of locomotor activity of freely moving rats. Brain Res Bull; 16: 377-381.

Bucy PC and Case TJ (1939). Tremor, physiologic mechanism and abolition by surgical means. Arch Neurol Psychiat; 41: 721-746.

Burchiel KJ (1995). Thalamotomy for movement disorders. Neurosurg Clin N $A m ; 6: 55-71$.

Burn DJ, Mark MH, Playford ED, et al (1992). Parkinson's Disease in twin studies with ${ }^{18}$ F-DOPA and positron emmission tomography. Neurology; 42:1894-1900.

Carroll C, Scott R, Davies LE, et al (1998). The pallidotomy debate. $\mathrm{Br} J$ Neurosurg; 12(2):146-150. 
Chapman CA, Yeomans JS, Blaha CD, et al (1997). Increased striatal dopamine efflux follows scopolamine administered systemically or to the tegmental pedunculopontine nucleus. Neuroscience; 76:177-186.

Charara A, Smith J, Parent A (1996). Glutamatergic inputs from the pedunculopontine nucleus to midbrain neurons in primates: Phaseolus vulgaris-leucoagglutinin anterograde labelling compared with postembedding glutamate and GABA immunohistochemistry. J Comp Neurol; 364:254-266.

Clarke CE, Sambrook MA, et al (1987). Levodopa induced dyskinesia and response fluctuations in primates rendered parkinsonian with MPTP. J Neurol Sci; 78: 273-280.

Clarke R and Horsley V (1906). On a method of investigating the deep ganglia and tracts of the central nervous system (cerebellum). BMJ; 2: 1799-1800.

Clarke RH (1920). Part I: Investigation of the central nervous system methods and instruments. The Johns Hopkins Hospital Reports (special volume); Baltimore: The Johns Hopkins Press.

Cooper I (1965). Surgical treatment of parkinsonism. Ann Rev Med; 16: 309330.

Cooper I and Bravo G (1958). Chemopallidectomy and chemothalamectomy. $J$ Neurosurg; 15: 244-250.

Cooper I, Gioino G, et al (1965). The cryogenic lesion. Confinia Neurol; 26: 161-177.

Cooper I. (1956). The neurosurgical alleviation of parkinsonism. Springfield, Illinois. Charles C Thomas. 
Cooper IS (1953). Ligation of the anterior choroidal artery for involuntary movements and parkinsonism. Psychia. Quart; 27: 317-319.

Cooper IS (1954). Intracerebral injection of procaine into the globus pallidus in hyperkinetic disorders. Science; 119: 417-418.

Cordeau J (1961). Microelectrode studies in monkeys with a postural tremor. Revue CanBiol; 20: 147-157.

Cotzias GP, Papavasiliou PS, et al (1969a). Long term effects of DOPA on parkinsonism: A proposal. Third symposium on Parkinson's disease, Ediburgh,London, Livingstone.

Cotzias G, Papvasiliou PS, et al (1969b). Modification of ParkinsonismChronic treatment with L-DOPA. NEJM; 280: 337-345.

Couper J (1837). On the effects of black manganese when inhaled into the lungs. British Annals of Medicine, Pharmacology, Vitamins, Statistics and General Science; 1:41-42.

Crossman AR, Mitchell IJ, Sambrook MA (1985). Regional brain uptakeof 2deoxyglucose in 1-methyl-4-phenyl-1,2,3,6-tetrahydropyridine (MPTP) induced parkinsonism in the macaque monkey. Neuropharmacology; 24:587591.

Deacon T, Schumacher J, Dinsmore J, et al (1997). Histological evidence of foetal pig neural cell survival after trensplantation into a patient with Parkinson's Disease. Nature Medicine; 3:350-353. 
Delmas-Marsalet P and Bogaert LV (1935). Sur un cas de myoclonies rythmiques continuees determinees par une intervention chirurgicale sur le tronc cerebral. Rev. Neurol; 64: 728-748.

DeLong M, Georgopoulos A, et al (1984). Functional organisation of the basal ganglia: contributions of single cell recording studies. Functions of the Basal Ganglia. London, Pitman: 64-82.

DeLong M (1990). Primate models of movement disorders of basal ganglia origin. Trends Neurosci; 13:281-285.

DePalma G, Mozzoni P, Mutti A, et al (1998). Case-control study of interactions between genetic and enviromental factors in Parkinson's Disease. The Lancet; 352:1986-1987.

Descartes R (1637). Discours de la methode. Leyden, Holland: Paris, GarnierFlammarion, 1966.

Duffy P and Tennyson V (1965). Phase and EM observations of Lewy bodies and melanin granules in the substantis nigra and locus coeruleus in Parkinson's disease. J Neuropath Exp Neurol; 24: 398-414.

Dupont E (1977). Epidemiology of parkinsonism: The Parkinson investigation, Arhus Denmark. Symposium on Parkinsonism. Denmark, Merck, Sharp and Dohme. 65-75.

Duvoisin R. (1989). Is there a Parkinson's disease? Disorders of Movement. London, Academic Press: 1-10. 
Duvoisin R, Barrett R, et al (1969). The use of L-Dopa in parkinsonism. Third symposium on Parkinson's disease. Edinburgh, London. Livingstone.

Ebin J (1949).“Combined lateral and ventral pyramidotomy in treatment of paralysis agitans. Arch Neurol Psychiat; 62: 27-47.

Ehringer H and Hornykiewicz O (1960). Verteilung von noradrenalin und dopamin (3-hydroxytyramin) im gehirn des menschen und ihr verhalten bei erkrankungen des extrapyramidalen systems. Klin Wochenschr; 38: 12361239.

Eidelberg E, Walden JG, Nguyen LH (1981). Locomotor control in Macaque monkeys. Brain; 104:647-663.

Elias SA, Palmer EP, KottHS, et al (1998). Transplantation of foetal porcine ventral mesencephalic cells for treatment of Parkinson's Disease. One year safety and efficacy results. Neurology; 50:A80.

Fager CA (1968). Evaluation of thalamic and subthalamic surgical lesions in the alleviation of Parkinson's disease. J Neurosurg;; 28: 145-149.

Favre J, Taha J, Nguyen T, et al (1996). A survey of current practice in North America. Neurosurgery; 39(4):883-890.

Fodstad H, Hariz M, Ljunggren B (1991). History of Clarke's stereotactic instrument. Stereotact Funct Neurosurg; 57:130-140.

Foester O (1927). Ueber die VorderseitenstrangdurchSchneidung. Arch $f$ Psychiat : 707-717. 
Foix M (1921). Les lesions anatomiques de la maladie de Parkinson. Revue Neurologique; 28: 593-600.

Forno L (1966). Pathology of parkinsonism : a preliminary reportof 24 cases. $J$ Neurosurg; 24: 266-271.

Galvez-Jiminez N, Lozano A, Duff J, et al (1996). Bilateral pallidotomy: pronounced amelioration of incapacitating levodopa induced dyskinesias but accompanying cognitive decline. Mov Disord; 11:242.

Garcia-Rill E (1986). The basal ganglia and the locomotor regions. Brain Res $\operatorname{Rev} ; 11: 47-63$.

Garcia-Rill E, Houser CR, et al (1987). Locomotion inducing sites in the vicinity of the pedunculopontine nucleus. Brain Res Bull; 18: 731-738.

Garcia-Rill E, Skinner RD (1987a). The mesencephalic locomotor region. I. Activation of a medullary projection site. Brain Res; 411:1-12.

Garcia-rill E, Skinner RD (1987b). The mesencephalic locomotor region. II. Projections to reticulospinal neurons. Brain Res; 411:13-20.

Garcia-Rill E, Skinner RD (1988). Modulation of rhythmic function in the posterior midbrain. Neuroscience; 27:639-654.

Garcia-Rill E, Skinner RD (1991). Modulation of rhythmic functions by the brainstem. In Shimamura M, Grillner S, Edgerton VR (eds). Neurobilolgical Basis of Locomotion. Tokyo. Japan Societies Scientific Press 137-158. 
Garcia-Rill E, Biedermann JA, Chambers T, et al (1995a). Mesopontine neurons in schizophrenia. Neuroscience; 66:321-335.

Garcia-Rill E, Reeses NB, Skinner RD (1995b). Arousal and locomotionfrom schizophrenia to narcolepsy. In Holstege G, Saper C (eds). The Emotional Motor System. Amsterdam. Elsevier Science.

Gildenberg P (1975). Survey of stereotactic and functional neurosurgery in the United States and Canada. Appl Neurophysiol; 38: 31-37.

Gill S, Heywood P (1997). Bilateral dorsolateral subthalamotomy for advanced Parkinson's disease. Lancet; 350:1224.

Golbe LI, Iorio G, Sanges G, et al (1996). Clinical genetic analysis of Parkinson's Disease in the contursi kindred. Ann Neurol; 40:767-775.

Goldman M, Ahlskig J, Kelly P (1992). The symptomatic and functional outcome of stereotactic thalamotomy for medically intractable essential tremor. J Neurosurg; 76:924-928.

Goldsmith M, Van der Kooy D (1988). Separate non-cholinetrgic descending projections and cholinergic ascending projections from the nucleus tegmenti pedunculopontis. Brain; 445:386-391.

Gordon EB (1965). Carbon monoxide encephalopathy. BMJ; 1:1232-1233.

Gould E, Woolf NJ, Butcher LL (1989). Cholinergic projections to the substantia nigra from the pedunculopontine and laterodorsal tegmental nuclei. Neuroscience; 28:611-623. 
Granata AR, Kitai ST (1991). Inhibitory substantia nigra inputs to the pedunculopontine neurons. Exp Brain Res; 86:459-466.

Grillner S (1985). Neurobiological basis of rhythmic motor acts in vertebrates. Science; 228:143-149.

Grinkler RR (1926). Parkinsonism following carbon monoxide poisoning. $J$ Nerv and Mental Disord; 64:18-28.

Grunwerg BS, Krein H, Krauthamer GM (1992). Somatosensory input and thalamic projection of pedunculopontine tegmental neurons. Neuroreport; 3:673-675.

Guela C, Schatz CR, Mesulam MM (1993). Differential localisation of NADPH-diaphorase and calbindin D-28k within the cholinergic neurons in the basal forebrain, striatum and brainstem in the rat, monkey, baboon and human. Neuroscience; 54:461-476.

Guiot G and Brion S (1953). Traitement de mouvments anormaux par la coagulation pallidale. $\operatorname{Rev}$ Neurol; 89: 578-580.

Guridi J, Herrero MT, Luquin MR, et al (1996). Subthalamotomy in parkinsonian monkeys: behavioural and biochemical analysis. Brain; 119:1717-1728.

Hallanger AE, Levey AI, Lee HJ, et al (1987). The origins of cholinergic and other subcortical afferents to the thalamus in the rat. J Comp Neurol; 262:105124. 
Hallanger AE, Wainer $\mathrm{BH}$ (1988). Ascending projections from the pedunculopontine tegmental nucleus and the adjacent mesopontine tegmentum in the rat. J Comp Neurol; 274:483-515.

Hammond C, Rouzaire-Dubois B, et al (1983). Anatomical and electrophysiological studies on the reciprocal projections between the subthalamic nucleus and the nucleus tegmenti pedunculopontinus in the rat. Neurosci; 9(1): 41-55.

Harnois C, Filion M (1982). Pallidofugal projections to the thalamus and midbrain: a quantatative antidromic activation study in monkeys and cats. Exp Brain Res; 47:277-285.

Hassler R (1938). Zur Pathologie der Paralysis agitans und des postenzephalitischen Parkinsonismus. Journal fur Psychologie und Neurologie; 48: 387.

Hassler R, Reichert T (1954). Indikationen und lokalisationmehod der gezielten himoperation. Inervenarzt; 25:441-447.

Hassler R, Reichert T (1958). Uber die symptomatik und operative behandling der extrapyramidalen bewegungsstroungen. Med Klin; 538:17-24.

Hassler R, Mundinger F, et al (1979). Stereotaxis in parkinson syndrome. Berlin, Heidelberg,New York. Springer Verlag. 
Herrero MT, Levy R, Ruberg R, et al (1996). Consequences of nigrostriatal denervation and L-dopa therapy on the expression of glutamic acid decarboxylase (GAD) messenger RNA in the pallidum. Neurology; 47: 219224.

Hikosaka O. Wurtz RH (1983). Visual and oculomotor functions of monkey substantia nigra pars reticulata. Relation of the substantia nigra to the superior colliculus. J Neurophysiol; 49:1285-201.

Hirsch EC, Graybiel AM, Duyckaerts C, et al (1987). Neuronal loss in the pedunculopontine tegmental nucleus in Parkinson's Disease and progressive supranuclear palsy. Pro Natl Acad Sci USA; 84:5976-5980.

Hitchcock E (1969). An apparatus for stereotactic spinal surgery. Lancet; 1: 705-706.

Hoehn MM, Elton RL (1985). Low dosages of bromocriptine added to levodopa in Parkinson's disease. Neurology; 35: 199-206.

Horsley V, Clarke R (1908). The structure and functions of the cerebellum examined by a new method. Brain; 31: 45-124.

Horsley VA (1909). The Linacre Lecture on the function of the so called "motor" area of the brain. BMJ; 2: 125-132.

Hughes B (1969). Evaluation of the subthalamic lesion in parkinsonism. Third symposium on Parkinson's disease. Edinburgh, London. Livingstone. 
Hylden JL, Hayashi H, Bennett GJ, et al (1985). Spinal lamina I neurons projecting to the parabrachial area of the cat midbrain. Brain Res; 336:195198.

Imperato A, Obinu M, Reibaud M, et al (1998). Riluzole prevents dyskinesias in parkinsonian marmosets. Abstracts of SFNS Annual Meeting 1998;24(1):763.

Iacono R, Shima F, Lonser R, et al (1995). The results, indications and physiology of posteroventral pallidotomy for patients with Parkinson;s Disease. Neurosurgery; 36:118-1125.

lizuka J (1975). Development of a stereotactic endoscopy of the ventricular system. Confin Neurol; 37: 141-149.

Inglis WL, Winn P (1995). The pedunculopontine nucleus: where the striatum meets the reticular formation. Prog Neurobiol; 47:1-29.

Jackson A, Crossman AR (1983). Nucleus tegmenti pedunculopontinus, efferent connections with special reference to the basal ganglia, studied in the rat by anterograde and retrograde transport of horse radish peroxidase. Neuroscience; 10(3): 725-765.

Janckovic J, Cardoso G, Grossman R, et al (1995). Outcome after stereotactic thalamotomy for Parkinson's, essential and other types of tremor. Neurosurgery; 37:680-686.

Jefferson G (1957). Sir Victor Horsley, 1857-1916. BMJ; 1:903-910. 
Jellinger K (1988). The pedunculopontine nucleus in Parkinson's Disease, progressive supranuclear palsy and Alzheimer's disease. J Neurol Neurosurg Psychiatr; 51:540-543.

Jellinger KA (1995). Effect of adding selegeline to levodopa in early, mild Parkinson's Disease. Causes of death need confirmation. BMJ; 311(7020):1602-1607.

Johannson F, Malm J et al (1997). Usefulness of pallidotomy in advanced Parkinson's disease. J Neurol Neurosurg Psychiatry; 62:125-132.

Jones BE (1991). Paradoxical sleep and its chemical/structural substrates in the brain. Neuroscience; 40:637-656.

Kandel E (1989). Functional and stereotactic neurosurgery. New York, London. Plenum Medical Book Company.

Kebabian J, Calne D (1979). Multiple receptors for dopamine. Nature; 277: 92-96.

Kelland MD, Asdourian D (1989). Pedunculopontine tegmental nucleusinduced inhibition of muscle activity in the rat. Behav Brain Res; 34:213-234.

Kessler II (1972). Epidemiologic studies of Parkinson's disease. II. A hospital based survey. Am J Epidemio;. 95: 308-318.

Kita H, Kitai ST (1987). Efferent projections of the subthalamic nucleus in the rat: light and electron microscopic analysis with the PHA-L method. J Comp Neurol; 260:435-452. 
Klawans HL, Stein RW, Tanner CM, et al (1982). A pure parkinsonian syndrome following acute carbon monoxide intoxication. Arch Neurol; 39:302-304.

Klemme RM (1940). Surgical treatment of dystonia, paralysis agitans and athetosis. Arch Neurol; 24:82-85.

Kojima J, Yamaji Y, Matsumara M, et al (1997). Excitotoxic lesions of the pedunculopontine tegmental nucleus produce contralateral hemiparkinsonism in the monkey. Neurosci Lett; 226(2):111-114.

Koller W, O'Hara R, et al (1986). Relationship of aging to Parkinson's disease. Advances in Neurology: 317-321.

Koller W, Long A, Tarsy D, et al (1997). High frequency chronic stimulation for the treatment of essential and parkinsonian tremor. Ann Neurol; 42:292299.

Kopyov O, Jacques D, Duma C, et al (1997). Microelectrode guided posteroventral medial radiofrequency pallidotomy for Parkinson's Disease. $J$ Neurosurg; 87:52-59.

Krack P, Limousin P, Benabid A, et al (1997). Chronic stimulation of subthalamic nucleus improves levodopa induced dyskinesias in Parkinson's Disease. Lancet; 350:1676.

Krack P, Pollack P, Limousin P, eta 1 (1998). Subthalamic nucleus or internal pallidal stimulation in young onset Parkinson's Disease. Brain; 121:451-452. 
Krack P, Pollack P, Limousin P, et al (1998). Opposite motor effects in pallidal stimulation in Parkinson's disease. Ann Neurol; 43:180-192.

Krauss J, Desalons J, Lai E, et al (1997). Microelectrode guided posteroventral pallidotomy for treatment of Parkinson's Disease: postoperative MRI image analysis. J Neurosurg; 87:358-367.

Krayenbuhl H, Wyss O, et al (1961). Bilateral thalamotomy and pallidotomy as treatment for bilateral parkinsonism. J. Neurosurg; 16: 429-444.

Lai YY, Siegel JM (1990). Muscle tone supression and stepping produced by stimulation of midbrain and rostral pontine reticular formation. $J$ Neurosci; 10:2727-2734.

Laitinen L, Vikki J (1973). Measurement of parkinsonian hypokinesia with Purdue Pegboard and motor reaction time tests. In : Siegfried J (ed) Parkinson's Disease, Vol 2. Berlin, Huber:185-192.

Laitinen LV (1985). Brain targets in surgery for Parkinson's disease:- Results of a survey of neurosurgeons. J. Neurosurg; 62: 349-351.

Laitinen L, Bergenheim A, Hariz M (1992). Leksell's posteroventral pallidotomy in the treatment of Parkinson's Disease. J Neurosurg; 76:53-61.

Lamarre Y, Cordeau J (1962). Central unit activity in monkeys with postural tremor. Fed Proc; 22: 1993.

Lance JR, Schwab, et al (1963). Action tremor and the cogwheel phenomenon in Parkinson's Disease. Brain; 86:95-110. 
Lavoie B, Parent A (1994a). Pedunculopontine nucleus in the squirrel monkey: distribution of cholinergic and monoaminergic neurons in the mesopontine tegmentum with evidence for the presence of glutamate in the cholinergic neurons. J Comp Neurol; 344:210-231.

Lavoie B, Parent A (1994b). Pedunculopontine nucleus in the squirrel monkey: projections to the basal ganglia as revealed by anterograde tracing methods. J Comp Neurol; 344:210-231.

Lavoie B, Parent A (1994c). Pedunculopontine nucleus in the squirrel monkey: cholinergic and glutamatergic projections to the substantia nigra. $J$ Comp Neurol; 344:232-241.

Lee HJ, Rye DB, Hallanger AE, et al (1998). Cholinergic vs. non-cholinergic efferents from the mesopontine tegmentum to the extrapyramidal motor system nuclei. J Comp Neurol; 275:469-492.

Lees AJ (1986). L-DOPA treatment and Parkinson's disease. Quart J of Med; 59(230): 535-547.

Lees AJ (1995). Comparison of therapeutic effects and mortality data of levodopa combined with selegeline in patients with early, mild Parkinson's Disease. Parkinson's Disease Research Group of the United Kingdom. BMJ; 311(7020):1602-1607.

Leksell L, Larsson B, et al (1960). Lesions in the depth of the brain produced by a beam of high energy protons. Acta Radiol; 54: 251-264. 
Leksell L, Leksell D, et al (1985). Stereotaxis and nuclear magnetic resonance. J Neurol Neurosurg Psych;: 48: 14-18.

Leriche (1912). Uber chirurgische Eingriffe bei Parkinsonischer Krankheit. Neurol. Zb;. 13: 1093-1096.

Lewy F (1913). Zur pathologischen Anatomie de Paralysis agitans. Ztschr Nervenheilk; 50: 50-55.

Li TM, Swash M, et al (1985). Morbidity and mortality in motor neurone disease: comparison with multiple sclerosis and Parkinson's disease: age and sex specific rates and cohort analyses. J Neurol Neurosurg Psych; 48: 320327.

Lieberman AN, Gopinathan G, et al (1984). Should dopamine agonists be given early or late? A review of nine years experience with bromocriptine. Can. J. Neurol. Sci; 11(Suppl. 1): 233-237.

Limousin P, Pollack P, Benazzouz A, et al (1995). Improvement in parkinsonian symptoms after bilateral subthalamic nucleus stimulatiom. Lancet; 345:91-95.

Limousin P, Pollack P, Benazzouz A, et al (1998). Effects of parkinsonian signs and symptoms of bilateral STN stimulation. Lancet; 345:91-95.

Lindvall O, Bjorklund S (1979). Dopaminergic innervation of the globus pallidus by collaterals from the nigrostriatal pathway. Brain Res; 172: 169173. 
Lindvall O (1989). Transplantation into the human brain: present status and future possibilities. J Neurol Neurosurg Psychiatr; (June, Suppl.): 39-54.

Lindvall O, Rehncrona S, et al (1989). Human fetal dopamine neurones grafted into the striatum in two patients with severe Parkinson's disease: A detailed account of methodology and a 6 month follow up. Arch. Neurol; 46: 615-631.

Lipkin L (1959). Cytoplasmic inclusions in ganglion cells associated with parkinsonian states. Am J Pathol; 35: 1117-1133.

Lopez-Lozano J, Bravo G, et al (1991). Grafting of perfused adrenal medullary tissue into the caudate nucleus of patients with Parkinson's disease. J Neurosurg; 75: 234-243.

Lozano A, Hutchinson W, Kiss Z, et al (1996). Methods for microelectrode guided posteroventral pallidotomy. $J$ Neurosurg; 84:194-202.

Lozano A, Lang A (1998). Pallidotomy for Parkinson's disease. Neurosurg Clin N Am; 9:325-336.

MacMahon DG, Bland R (1996). Effect of adding selegeline to levodopa in early, mild Parkinson's disease. Selegeline is effective and safe in early stages. BMJ; 311(7020):1602-1607.

Madrazo I, Drucker-Colin R, et al (1987). Open microsurgical autograft of adrenal medulla to the right caudate nucleus in two patients with intractable Parkinson's disease. NEJM; 316: 831-834. 
Maki-Ikola O, Kilkku O, Iieinonen E (1995). Effect of adding selegeline to levodopa in early, mild Parkinson's Disease. Other studies have not shown increased mortality. $B M J ; 311(7020): 1602-1607$.

Malessa S, Hirsch EC, Cervera p, et al (1991). Progressive supranuclear palsy: loss of choline-acetyltransferase-like immunoreactive neurons in the pontine reticular formation. Neurology; 41(10):1593-1597.

Marsden CD (1969). Extending the use of anti-cholinergic drugs in Parkinson's disease. Third symposium on Parkinson's disease. Edinburgh, London, Livingstone. 195-199.

Marsden CD (1987). Parkinson's disease in twins. J Neurol Neurosurg Psychiatr; 50:105-106.

Masdeu JC, Alampur U, Cavalaiere R, et al (1994). Astasia and gait failure with damage of the pontomesencephalic locomotor region. Ann Neurol; 35:619-621.

Mesulam MM, Mufson EJ, Wainer BH, et al (1983). Central cholinergic pathways in the rat: an overview based on an altered nomenclature. Neuroscience; 10:1185-1201.

Mesulam MM, Guela C, Bothwell MA, et al (1989). Human reticular formation: cholinergic neurons of the perunculopontine and laterodorsal tegmental nuclei and some cytochemical comparisons to forebrain cholinergic neurons. J Comp Neurol; 281:611-633. 
Meyers R (1940). A surgical procedure for post-encephalitic tremor, with notes on the physiology of the pre-motor fibres. Arch Neurol Psychiatr; 44: 455-459.

Meyers RM (1942). The modification of alternating tremors, rigidity and festination by surgery of the basal ganglia. A Nerv Ment Disord; 21:602-665.

Meyers R (1951). Surgical experiments in the therapy of certain " extrapyramidal" diseases, a current evaluation. Acta Psych Neurol; 67: 1-42.

Meyers R, Sweeney D B, et al (1950). Hemiballismus: aetiology and surgical treatment. J Neurol Neurosurg Psychiatr;. 13: 115-126.

Meyers R, Fry W, et al (1959). Early experiences with ultrasonic irradiation of the pallidofugal and nigral complexes in hyperkinetic and hypertonic disorders. J Neurosurg; 16: 32-54.

Milner KL, Mogenson GJ (1988). Electrical and chemical activation of the mesencephalic and subthalamic locomotor regions in freely moving rats. Brain Res; 452: 273-285.

Mitchell IJ, Clarke CE, Boyce S, et al (1989). Neural mechanisms underlying parkinsonian symptoms based upon regional uptake of 2-deoxyglucose in monkeys exposed to MPTP. Neuroscience; 32: 213-226.

Mogenson GJ, Wu M (1988). Differential effects on locomotor activity of injections of procaine into mediodorsal thalamus and pedunculopontine nucleus. Brain Res Bull; 20: 241-246. 
Moon-Edley S, Graybiel AM (1983). The afferent and efferent connections of the feline nucleus tegmenti pedunculopontis pars compacta. J Comp Neurol; $217: 187-215$

Munro-Davies LE, Gregory R, Squires W, et al (1999). Lateral pallidotomy exacerbates akinesia in the parkinsonian patient: a Case report. $J$ Clin Neuroscience; 6(6):

Mutch WJ, Dingwall-Fordyce I, et al (1986). Parkinson's disease in a Scottish city. $B M J$;292: 534-536.

Narabayashi H, Hirotaro, Okuma t, et al (1953). Procaine oil blocking of the globus pallidus for the treatment of rigidity and tremor of parkinsonism. Proc Japan Acad; 29:310-318.

Narabayashi H, Okuma T, et al (1956). Procaine oil blocking of the globus pallidus. Arch Neurol Psychiatr; 75: 36-48.

Narabayashi $\mathrm{H}$ et al (1991). Analysis of L-threo-3,4-dihydroxyphenylserine effect on motor and psychological symptoms in Parkinson's Disease. No To Shinkei; 43(3):263-268.

Noda T, Oka H (1986). Distribution and morphology of tegmental neurons receiving nigral inhibitory inputs in the cat: an intracellular HRP study. $J$ Comp Neurol; 244:254-266.

Nutt JG, Woodward WR, et al (1984). The on-off effect in Parkinson's disease. $N E J M ; 310: 483-488$. 
Nyberg P, Nordberg A, et al (1983). Dopaminergic deficiency is more pronounced in putamen than nucleus caudatus in Parkinson's disease. Neurochem Pathol; 1: 193-202.

Ogura M, Nakao N, Nakai K, et al (1997). Firing activity of the basal ganglia and pedunculopontine nucleus in rats with nigrostriatal lesions. Stereotact Funct Neurosurg; 67:80-81.

Oliver L (1950). Surgery in Parkinson's disease, complete section of the lateral column of the spinal cord for tremor. Lancet; 1: 847-848.

Olszewski J, Baxter D (1982). Cytoarchitecture of the human brainstem. $\left(2^{\text {nd }}\right.$ ed.). Basel, Karger: 195.

Pahwa R, Wilkinson S, Smith D, et al (1997). High frequency stimulation of the globu spallidus for the treatment of Parkinson's Disease. Neurology; 49:249-253.

Papanastassiou V, Rowe J, Scott R, et al (1998). Use of the Radionics Imagefusion ${ }^{\mathrm{TM}}$ and Stereoplan ${ }^{\mathrm{TM}}$ programs for target localization in functional neurosurgery. J Clin Neuroscience; 5(1):28-32.

Parent A, Hazrati LN (1993). Anatomical aspects of information processing in primate basal ganglia. Trends Neuroscience; 16:308-309.

Piccini P, Morrish PK, Turjanski N, et al (1997). Dopaminergic function in familial Parkinson's Disease: a clinical and ${ }^{18}$ F-DOPA positron emmission tomography study. Ann Neurol; 41:222-229. 
Pincus JH, Barry K (1987). Influence of dietary protein on motor fluctuations in Parkinson's disease. Arch Neurol; 44: 270-272.

Pollock LJ, Davis L (1930). Muscle tone in parkinsonian states. Arch Neurol; 23: 303.

Pollack O, Benabid AL, Limousin P, et al (1998). Subthalamic nucleus stimulation alleviates akinesia and rigidity in Parkinson's Disease. Lancet; 351:340-341.

Polymeropoulus M, Lavedan C, Leroy E, et al (1996). Mapping of a gene for Parkinson's Disease to chromosome 4q21-23. Science; 274:1197-1199.

Polymeropoulus M, Lavedan C, Leroy E, et al (1997). Mutation in the $\alpha$ synuclein gene in families with Parkinson's Disease. Science; 276:2045-2047.

Putnam TJ (1938). Relief from unilateral paralysis agitans by section of the pyramidal tract. Arch Neurol; 40: 1049-1050.

Putnam TJ (1940). Treatment of unilateral paralysis agitans by section of the lateral pyramidal tract. Arch Neurol; 44: 950-976.

Quinn N, Marsden CD, et al (1982). Complicated response fluctuations in Parkinson's disease. Lancet ; 2: 412-415.

Rajput A (1984). Etiology of Parkinson's disease: Environmental factors.

Rajput AH, Uitti RJ, et al (1986). Early onset Parkinson's disease and childhood environment. Advances in Neurology; 295-297. 
Rand R, Jacques D, Melbye R, et al (1993). Gamma-knife thalamotomy and pallidotomy in patients with movement disorders: preliminary results. Stereotact Funct Neurosurg; 61(1):65-91.

Reese NB, Garcia-Rill E, Skinner RD (1995). The pedunculopontine nucleusauditory input, arousal and pathophysiology. Prog in Neurobiol; 42:105-133.

Reichart W, Doolittle J, et al (1982). Vestibular dysfunction in Parkinson's disease. Neurology; 32: 1133-1138.

Riley D, Lang AE (1988). Practical application of a low protein diet for Parkinson's disease. Neurology; 38: 1026-1031.

Rowe J, Davies L, Scott R, et al (1999). Surgical complications of functional neurosurgery treating movement disorders: Results with anatomical localisation. J Clin Neuroscience; 6(1):36-37.

Rye DB, Saper CB, Lee HJ, et al (1987). Pedunculopontine nucleus of the rat: cytoarchitecture, cytochemistry and some extrapyramidal connections of the mesopontine tegmentum. J Comp Neurol; 259:483-528.

Rye DB, Lee HJ, Saper CB, et al (1988). Medullary and spinal efferents of the pedunculopontine tegmental nucleus and adjacent mesopontine tegmentum in the rat. Comp Neurol; 269:315-341.

Rye DB, Vitek J, Bakay RAE, et al (1995). Termination of paalidfugal pathways in man. Neurosci Abst; 21:676. 
Sacks O (1991). Awakenings. London, Picador.

Scarnati E, Haidu F, Pacitti, et al (1988). An EM and golgi study on the connection between the nucleus tegmenti pedunculopontinus and the pars compacta of the substantia nigra in the cat. J Hironforsch; 1:95-105.

Scarnati E (1996). The pedunculopontine nucleus and related structures: Functional organisation. In: Obeso JA, dElONG mr, Ohye $\mathrm{CH}$, Marsden $\mathrm{CD}$, eds. The Basal Ganglia and Surgical Treatment of Parkinson's Disease. New York. Raven Press.

Schoenberg,BS, et al (1988). Comparison of the prevalence of Parkinson's disease in black populations in the rural United States and in rural Nigeria: door to door community studies. Neurology; 38: 645-646.

Schuur PH, Merrington WR (1978). The Horsley-Clarke srereotactic apparatus. Br J Surg; 65:33-36.

Schuuman R, de Bie R, Speelman J, et al (1997). Posteroventral pallidotomy for movement disorders. Acta Neurochir Suppl Wien; 68:14-27.

Scott R, Gregory R, Hines N, et al (1998). Neuropsychological, neurological and functional outcome following pallidotomy for Parkinson's disease: a consecutive series of 8 simultaneous bilateral and 12 unilateral procedures. Brain; 121:659-675.

Shima F, Ishidok K, Sun S, et al (1996). Surgical control of akinesia in Parkinson's disease. Neurol; 36(1):55-61. 
Shink E, Sidibe M, Smith Y (1997). Efferent connections of the internal globus pallidus in the squirrel monkey: II. Topography and synaptic organisation of pallidal efferents to the pedunculopontine nucleus. J Comp Neurol; 382:348-363.

Shiromani PJ, Armstrong DM, Berkowitz A, et al (1988a). Distribution of choline acetyltransferase immunoreactive somata in the feline brainstem: implicators for REM sleep generation. Sleep; 11:1-16.

Shiromani PJ, Armstrong DM, Gillin JC (1988b). Cholinergic neurons from the dorsolateral pons project to the medial pons: a WGA-HRP and choline acetyltransferase immunohistochemical study. Neuroscience; 95:19-23.

Shoulson I, Glaubiger GA, et al (1975). On-off response: Clinical and biochemical correlations during oral and intravenous L-DOPA administration in parkinsonian patients. Neurology; 25: 1144-1148.

Siegfried J, Lippitz B (1993). Bilateral chronic electrostimulation of venteroposterolateral pallidu: a new therapeutic approach for alleviating all parkinsonian symptoms. Neurosurgery; 35:1126-1130.

Silva MT, Watts PM, Jenner P (1995). Effect of adding selegeline to levodopa in early, mild Parkinson's Disease. Parkinson's Disease is rarely a primary cause of death. BMJ; 311(7020):1602-1607.

Skinner RD, Kinjo N, Henderson V, et al (1990). Locomotor projections from the pedunculopontine tegmental nucleus to the spinal cord. Neurireport; 1:183-186. 
Sofroniew M, Priestley JV, Consolazione A, et al (1985). Cholinergic projections from the midbrain and pons to the thalamus in the rat, identified by combined retrograde tracing and choline acetyltransferase immunocytochemistry. Brain Res; 213-223.

Spann BM, Grofova I (1991). Nigropedunculopontine projection in the rat: an anterograde study with Phaseolus vulgaris-Leucoagglutinin (PHA-L). J Comp Neurol; 311:375-388.

Spencer PS, Nunn PB, et al (1987). Guam amyotrophic lateral sclerosisParkinsonism dementia linked to a plant excitant neurotoxin. Science; 237: $517-522$.

Spiegel EA, Wycis HT, et al (1947). Stereotaxic apparatus for operations on the human brain. Science; 106: 349-350.

Steininger TL, Rye DB, Wainer BH (1992). Afferent projections to the cholinergic pedunculopontine tegmental nucleus and adjacent midbrain extrapyramidal area in the albino rat. I. Retrograde tracing studies. J Comp Neurol; 321:515-543.

Steinenger TL, Wainer BH, Rye DB (1997). Ultrastructural study of cholinergic and non-cholinergic neurons in the pars compacta of the rat pedunculopontine tegmental nucleus. J Comp Neurol; 382:285-301.

Steriade M, Pare D, et al (1988). Projections of cholinergic and noncholinergic neurones of the brain stem core to relay and associational thalamic nuclei in the cat and macaque monkey. Neurosci; 25(1): 47-67. 
Stibe C, Lees A, et al (1987). Subcutaneous imfusion of apomorphine and lisuride in the treatment of parkinsonian on-off fluctuations. Lancet; 1: 871.

Svennilson E, Torvik A, Lowe R, et al (1960). Treatment of parkinsonism by stereotactic thermal lesions in the pallidal region. Acta Psychiatr Scand; $35: 358-377$

Takada M (1995). Descending pathways of the basal ganglia. Kaibogaku Zanshi; 70(4):289-293.

Takakusaki K, Shiroyama T, Yamamoto T, et al (1996). Cholinergic and noncholinergic tegmental pedunculopontine projection neurons in rats revealed by intracellular labelling. J Comp Neurol; 371:345-361.

Tanji J, Kurata K (1983). Functional organisation of the supplementary motor cortex. In: Motor control mechanisms in health and disease. New York, Raven Press: 421-431.

Tasker R, Munz M, Junn F, et al (1997). Deep brain stimulation and thalamotomy for tremor compared. Acta Neurochir Suppl; 68:49-53.

Tretiakoff C (1919). Contribution a etude de l'anatomie pathologique du locus niger de Soemmering avec quelques deductions relatives a la pathogenie des troubles du tonus musculaire et de la maladie de Parkinson. Paris.

Trennier V, Fogel W, Kronenbuerger M, et al (1997). Pallidal stimulation: an alternative to pallidotomy. J Neurosurg; 87:700-705. 
Uematsu S, Rosenbaum A (1987). Magnetic resonance planned thalamotomy followed by X-ray/CT guided thalamotomy. Acta Neurochir Suppl; 39: 21-24.

Van Buren J, McCubbin D (1962). An outline atlas of the human basal ganglia with estimation of anatomical variants. J Neurosurg; 19: 811-839.

Viana Di Prisco, Pearlstein E, Robitaille R, et al (1997). Role of sensory evoked NMDA plateau potentials in the initiation of locomotion. Science; 278:1122-1125.

Vila M, Herrero MT, Levy R, et al (1996). Consequences of nigrostriatal denervation on the $\gamma$-aminabutyric acidic neurons of the substantia nigra pars reticulata and superior colliculus in parkinsonian syndrome. Neurology; 46:802-809.

Von Economo C (1931). The sequelae of encephalitis lethargica. In Newman $\mathrm{K}$ (ed). Encephalitis lethargica and its sequelae and treatment. Oxford University Press, London:105-106.

Vaughan J, Durr A, Tassin J, et al (1998). The $\alpha$-synuclein Ala53Thr mutation is not a common cause of familial Parkinson's disease: a study of 230 European cases. Ann Neurol; 44(2):270-273.

Walker A, Burton C (1966). Radiofrequency telethermocoagulation. JAMA; 197: 108-112.

Walker AE (1952). Cerebral pedunculotomy for the relief of involuntary movements. J Nerv Ment Dis; 116: 766-775. 
Ward CD, Duvoisin RC, et al (1983). Parkinson's disease in 65 pairs of twins and a set of quadruplets. Neurology; 33: 815-824.

Whittier J (1947). Ballism and the subthalamic nucleus : Review of the literature and study of of thirty cases. Arch Neurol Psychiat; 58: 672-692.

Winn P, Brown VJ, Inglis WL (1997). On th erelationship between the striatum and the pedunculopontine tegmental nucleus. Critical Rev in Neurobiol; 11:241-261.

Woolf NJ, Butcher LL (1986). Cholinergic systems in the rat brain. III. Projections from the pontomesencephalic locomotor tegnentum to the thalamus, tectum, basal ganglia and basal forebrain. Brain Res Bull; 16:603637.

Wszolek ZK, Pfeiffer B, Fulgham JR, et al (1995). Western Nebrasks family (family D) with autosomal dominant parkinonism. Neurology; 45:82-87.

Yong V, Perry T (1986). Depletion of glutathione in brain stem of mice caused by MPTP is prevented by antioxidant pretreatment. Neurosci Lett; 63: 56-70.

Young RF, Tronner V, Rinaldi PC (1992). Chronic stimulation of the Kolliker-Fuse nucleus region for relief of intractable pain in humans. $J$ Neurosurg; 76:979-985.

Ziegler L (1928). Follow up studies in persons who have had epidemic encephalitis. JAMA; 91:138-141. 
Zilker T, Fogt F, et al (1988). No parkinsonism after acute Paraquat poisoning. Klin Wochenschr; 66(November): 1138-1141.

Zwieg RM, Jankel WR, et al (1989). The pedunculopontine nucleus in Parkinson's disease. Ann Neurol; 26: 41-46. 
APPENDIX 1 
Calculation of Left PPN Co-Ordinates In Monkey 1.

ELECTRODE ZERO : $\quad$ AP $=85.0$

(i.e.tip-to-tip with posterior pin) Vert $=7.3$

MIDLINE AT SAGGITTAL SINUS: Lat $=44.5$

MAGNIFICATION : Actual distance between pin tips $=10 \mathrm{~mm}$

$\mathrm{X}$-ray distance between pin tips $=11 \mathrm{~mm}$

Magnification factor $=11 / 10=1.1$

X-RAY AC-PC DISTANCE [cm] $\quad: 1.35$

ACTUAL AC-PC DISTANCE[cm] $\quad: 1.35 / 1.1=1.23$

ENLARGEMENT FACTOR OFF ATLAS: 1.23

TARGET FROM ATLAS $\quad: 6 \mathrm{~mm}$ below PC

$2 \mathrm{~mm}$ lateral to midline

TARGET CORRECTED OFF ATLAS : 6 × $1.23=7.4$ below PC

TARGET ON X-RAY $\quad: 7.4 \times 1.1=8.1$ below PC

$2 \times 1.23=2.5$ lateral to midline

2.5 lateral to midline

TARGET RELATIVE TO POSTERIOR PIN ON X-RAY:

$\mathrm{AP}=2.5 \mathrm{~mm}$ Posterior

Vert $=6 \mathrm{~mm}$ Inferior

ACTUAL TARGET RELATIVE TO POSTERIOR PIN:

$\mathrm{AP}=2.5 / 1.1=2.3 \mathrm{~mm}$ POSTERIOR

Vert $=6 / 1.1=5.5 \mathrm{~mm}$ INFERIOR

\section{LEFT PPN CO-ORDINATES}

AP $\quad 85.0-2.3=\underline{\mathbf{8 2 . 7}}$

Lat $\quad 44.5-2.5=\underline{\mathbf{4 2 . 0}}$

Vert $\quad 7.3-5.5=\underline{\mathbf{1 . 8}}$ 
Calculation of Right PPN Co-Ordinates In Monkey 1.

ELECTRODE ZERO : $\quad$ AP $=81.0$

(i.e.tip-to-tip with posterior pin) Vert $=3.0$

MIDLINE AT SAGGITTAL SINUS: Lat $=42.0$

MAGNIFICATION : Actual distance between pin tips $=10 \mathrm{~mm}$ $\mathrm{X}$-ray distance between pin tips $=12 \mathrm{~mm}$ Magnification factor $=12 / 10=1.2$

X-RAY AC-PC DISTANCE [cm]

ACTUAL AC-PC DISTANCE[cm]

ENLARGEMENT FACTOR OFF ATLAS: 1.23

TARGET FROM ATLAS

: $6 \mathrm{~mm}$ below PC

$2 \mathrm{~mm}$ lateral to midline

TARGET CORRECTED OFF ATLAS : $6 \times 1.23=7.4$ below PC

TARGET ON X-RAY

$2 \times 1.23=2.5$ lateral to midline

: $7.4 \times 1.2=8.9$ below PC

2.5 lateral to midline

TARGET RELATIVE TO POSTERIOR PIN ON X-RAY:

$$
\begin{aligned}
& \mathrm{AP}=5 \mathrm{~mm} \text { Posterior } \\
& \text { Vert }=13 \mathrm{~mm} \text { Superior }
\end{aligned}
$$

ACTUAL TARGET RELATIVE TO POSTERIOR PIN:

$\mathrm{AP}=5 / 1.2=4.1 \mathrm{~mm}$ POSTERIOR

Vert $=13 / 1.2=10.8 \mathrm{~mm}$ SUPERIOR

\section{RIGHT PPN CO-ORDINATES}

AP $\quad 81.0-4.1=\underline{76.9}$

Lat $\quad 42.0+2.5=\underline{44.5}$

Vert $\quad 3.0-10.8=\underline{\mathbf{1 3 . 8}}$ 
Calculation of Left PPN Co-Ordinates In Monkey 2.

ELECTRODE ZERO : $\quad$ AP $=69.4$

(i.e.tip-to-tip with posterior pin) $\quad$ Vert $=23.0$

MIDLINE AT SAGGITTAL SINUS: Lat $=61.6$

MAGNIFICATION : Actual distance between pin tips $=10 \mathrm{~mm}$ $\mathrm{X}$-ray distance between pin tips $=11 \mathrm{~mm}$ Magnification factor $=11 / 10=1.1$

X-RAY AC-PC DISTANCE [cm] $\quad: 1.3$

ACTUAL AC-PC DISTANCE[cm] $\quad: 1.3 / 1.1=1.18$

ENLARGEMENT FACTOR OFF ATLAS: 1.2

TARGET FROM ATLAS $\quad: 6 \mathrm{~mm}$ below PC

$2 \mathrm{~mm}$ lateral to midline

TARGET CORRECTED OFF ATLAS : 6 x $1.2=7.2$ below PC

TARGET ON X-RAY

$2 \times 1.2=2.4$ lateral to midline

: $7.2 \times 1.1=7.9$ below PC

2.4 lateral to midline

TARGET RELATIVE TO POSTERIOR PIN ON X-RAY:

$\mathrm{AP}=1.5 \mathrm{~mm}$ Anterior

Vert $=7 \mathrm{~mm}$ Superior

ACTUAL TARGET RELATIVE TO POSTERIOR PIN:

$\mathrm{AP}=1.5 / 1.1=1.4 \mathrm{~mm}$ ANTERIOR

Vert $=7 / 1.1=6.4 \mathrm{~mm}$ SUPERIOR

\section{LEFT PPN CO-ORDINATES}

AP $\quad 69.4-2.3=\underline{70.8}$

Lat $\quad 61.6-2.4=\underline{\mathbf{5 9 . 2}}$

Vert $\quad 23.0+6.4=\underline{\mathbf{2 9 . 4}}$ 
Calculation of Right PPN Co-Ordinates In Monkey 2.

ELECTRODE ZERO : $\quad$ AP $=76.4$

(i.e.tip-to-tip with posterior pin) Vert $=4.5$

MIDLINE AT SAGGITTAL SINUS: Lat $=65.2$

MAGNIFICATION : Actual distance between pin tips $=10 \mathrm{~mm}$ $\mathrm{X}$-ray distance between pin tips $=11 \mathrm{~mm}$ Magnification factor $=11 / 10=1.1$

X-RAY AC-PC DISTANCE [cm] $\quad: 1.3$

ACTUAL AC-PC DISTANCE[cm] $\quad: 1.3 / 1.1=1.18$

ENLARGEMENT FACTOR OFF ATLAS: 1.2

TARGET FROM ATLAS $\quad: 6 \mathrm{~mm}$ below PC

$2 \mathrm{~mm}$ lateral to midline

TARGET CORRECTED OFF ATLAS : 6 x $1.1=7.2$ below PC

$2 \times 1.1=2.4$ lateral to midline

TARGET ON X-RAY

: $7.2 \times 1.1=7.9$ below PC

2.4 lateral to midline

TARGET RELATIVE TO POSTERIOR PIN ON X-RAY:

$\mathrm{AP}=3.5 \mathrm{~mm}$ Posterior

Vert $=17.5 \mathrm{~mm}$ Superior

ACTUAL TARGET RELATIVE TO POSTERIOR PIN:

$\mathrm{AP}=3.5 / 1.1=3.2 \mathrm{~mm}$ POSTERIOR

Vert $=17.5 / 1.1=15.9 \mathrm{~mm}$ SUPERIOR

\section{RIGHT PPN CO-ORDINATES}

AP $\quad 76.4-3.2=\underline{73.2}$

Lat $\quad 65.2+2.4=\underline{67.6}$

Vert $\quad 4.5+15.9=\underline{\mathbf{2 0 . 4}}$ 
Calculation of Left PPN Co-Ordinates In Monkey 3.

ELECTRODE ZERO : $\quad$ AP $=75.6$

(i.e.tip-to-tip with posterior pin) Vert $=18.0$

MIDLINE AT SAGGITTAL SINUS: Lat $=58.7$

MAGNIFICATION : Actual distance between pin tips $=10 \mathrm{~mm}$

$\mathrm{X}$-ray distance between pin tips $=11 \mathrm{~mm}$

Magnification factor $=11 / 10=1.1$

X-RAY AC-PC DISTANCE [cm] $\quad: 1.49$

ACTUAL AC-PC DISTANCE[cm] $\quad: 1.49 / 1.1=1.35$

ENLARGEMENT FACTOR OFF ATLAS: 1.35

TARGET FROM ATLAS $\quad: 6 \mathrm{~mm}$ below PC

$2 \mathrm{~mm}$ lateral to midline

TARGET CORRECTED OFF ATLAS : $6 \times 1.35=8.1$ below PC

TARGET ON X-RAY

$2 \times 1.35=2.7$ lateral to midline

: $8.1 \times 1.1=8.9$ below PC

2.7 lateral to midline

TARGET RELATIVE TO POSTERIOR PIN ON X-RAY:

$\mathrm{AP}=2.7 \mathrm{~mm}$ Anterior

Vert $=11.8 \mathrm{~mm}$ Superior

ACTUAL TARGET RELATIVE TO POSTERIOR PIN:

$\mathrm{AP}=2.7 / 1.1=2.5 \mathrm{~mm}$ ANTERIOR

Vert $=11.8 / 1.1=10.7 \mathrm{~mm}$ SUPERIOR

\section{LEFT PPN CO-ORDINATES}

AP $\quad 75.6+2.5=\underline{78.1}$

Lat $\quad 58.7-2.7=\underline{\mathbf{5 6 . 0}}$

Vert $\quad 18.0+10.7=\underline{\mathbf{2 8 . 7}}$ 
Calculation of Right PPN Co-Ordinates In Monkey 3.

ELECTRODE ZERO : $\quad$ AP $=78.6$

(i.e.tip-to-tip with posterior pin) Vert $=12.6$

MIDLINE AT SAGGITTAL SINUS: Lat $=57.4$

MAGNIFICATION : Actual distance between pin tips $=10 \mathrm{~mm}$ $\mathrm{X}$-ray distance between pin tips $=11 \mathrm{~mm}$ Magnification factor $=11 / 10=1.1$

X-RAY AC-PC DISTANCE [cm] $\quad: 1.49$

ACTUAL AC-PC DISTANCE[cm] $\quad: 1.49 / 1.1=1.35$

ENLARGEMENT FACTOR OFF ATLAS: 1.35

TARGET FROM ATLAS $\quad: 6 \mathrm{~mm}$ below PC $2 \mathrm{~mm}$ lateral to midline

TARGET CORRECTED OFF ATLAS : 6 x $1.35=8.1$ below PC $2 \times 1.35=2.7$ lateral to midline

TARGET ON X-RAY : $8.1 \times 1.1=8.9$ below PC

2.7 lateral to midline

TARGET RELATIVE TO POSTERIOR PIN ON X-RAY:

$\mathrm{AP}=2.6 \mathrm{~mm}$ Posterior

Vert $=11.3 \mathrm{~mm}$ Superior

ACTUAL TARGET RELATIVE TO POSTERIOR PIN:

$\mathrm{AP}=2.6 / 1.1=2.4 \mathrm{~mm}$ POSTERIOR

Vert $=11.3 / 1.1=12.1 \mathrm{~mm}$ SUPERIOR

\section{RIGHT PPN CO-ORDINATES}

AP $\quad 78.6-2.4=\underline{76.2}$

Lat $\quad 57.4+2.7=\underline{60.1}$

Vert $\quad 12.6+12.1=\underline{\mathbf{2 4 . 7}}$ 
Calculation of Bilateral PPN Co-Ordinates In Monkey 4.

ELECTRODE ZERO : $\quad$ AP $=66.2$

(i.e.tip-to-tip with posterior pin) Vert $=7.8$

MIDLINE AT SAGGITTAL SINUS: Lat $=36.6$

MAGNIFICATION : Actual distance between pin tips $=10 \mathrm{~mm}$

$\mathrm{X}$-ray distance between pin tips $=11 \mathrm{~mm}$

Magnification factor $=11 / 10=1.1$

X-RAY AC-PC DISTANCE [cm] $\quad: 1.4$

ACTUAL AC-PC DISTANCE[cm] $\quad: 1.4 / 1.1=1.3$

ENLARGEMENT FACTOR OFF ATLAS: 1.3

TARGET FROM ATLAS $\quad: 6 \mathrm{~mm}$ below PC

$2 \mathrm{~mm}$ lateral to midline

TARGET CORRECTED OFF ATLAS : $6 \times 1.3=7.8$ below PC

$2 \times 1.3=2.6$ lateral to midline

TARGET ON X-RAY

: $7.8 \times 1.1=8.6$ below PC

2.6 lateral to midline

TARGET RELATIVE TO POSTERIOR PIN ON X-RAY:

$\mathrm{AP}=3.0 \mathrm{~mm}$ Anterior

Vert $=7.0 \mathrm{~mm}$ Superior

ACTUAL TARGET RELATIVE TO POSTERIOR PIN:

$\mathrm{AP}=3.0 / 1.1=2.7 \mathrm{~mm}$ ANTERIOR

Vert $=7.0 / 1.1=6.3 \mathrm{~mm}$ SUPERIOR

LEFT CO-ORDINATES

AP $\quad 66.2+2.7=\underline{\mathbf{6 8 . 9}}$

Lat $\quad 36.6-2.6=\underline{\mathbf{3 4 . 0}} \quad 36.6+2.6=\underline{\mathbf{3 9 . 2}}$

Vert $\quad 7.8+6.3=\underline{\mathbf{1 4 . 1}}$ 
Calculation of Bilateral PPN Co-Ordinates In Monkey 5.

ELECTRODE ZERO : $\quad$ AP $=78.7$

(i.e.tip-to-tip with posterior pin) Vert $=20.2$

MIDLINE AT SAGGITTAL SINUS: Lat $=39.3$

MAGNIFICATION : Actual distance between pin tips $=10 \mathrm{~mm}$ $\mathrm{X}$-ray distance between pin tips $=11 \mathrm{~mm}$ Magnification factor $=11 / 10=1.1$

X-RAY AC-PC DISTANCE [cm] $\quad: 1.3$

ACTUAL AC-PC DISTANCE[cm] $\quad: 1.3 / 1.1=1.18$

ENLARGEMENT FACTOR OFF ATLAS: 1.18

TARGET FROM ATLAS $\quad: 6 \mathrm{~mm}$ below PC

$2 \mathrm{~mm}$ lateral to midline

TARGET CORRECTED OFF ATLAS : 6 x $1.18=7.1$ below PC

TARGET ON X-RAY

$2 \times 1.18=2.4$ lateral to midline

: $7.1 \times 1.1=7.8$ below PC

2.4 lateral to midline

TARGET RELATIVE TO POSTERIOR PIN ON X-RAY:

$\mathrm{AP}=4.0 \mathrm{~mm}$ Posterior

Vert $=18.0 \mathrm{~mm}$ Inferior

ACTUAL TARGET RELATIVE TO POSTERIOR PIN:

$\mathrm{AP}=4.0 / 1.1=3.6 \mathrm{~mm}$ POSTERIOR

Vert $=18.0 / 1.1=16.3 \mathrm{~mm}$ INFERIOR

LEFT CO-ORDINATES

AP

$78.7-3.6=\underline{75.1}$

Lat

$32.6-2.4=\underline{\mathbf{3 0 . 2}}$

$32.6+2.4=\underline{\mathbf{3 5 . 0}}$

Vert

$20.2-16.3=\underline{\mathbf{3 . 9}}$ 
Calculation of Bilateral PPN Co-Ordinates In Monkey 6.

ELECTRODE ZERO : $\quad$ AP $=74.9$

(i.e.tip-to-tip with posterior pin) Vert $=23.8$

MIDLINE AT SAGGITTAL SINUS: Lat $=58.0$

MAGNIFICATION : Actual distance between pin tips $=10 \mathrm{~mm}$ $\mathrm{X}$-ray distance between pin tips $=11 \mathrm{~mm}$ Magnification factor $=11 / 10=1.1$

X-RAY AC-PC DISTANCE [cm] $\quad: 1.49$

ACTUAL AC-PC DISTANCE[cm] $\quad: 1.49 / 1.1=1.35$

ENLARGEMENT FACTOR OFF ATLAS: 1.35

TARGET FROM ATLAS $\quad: 6 \mathrm{~mm}$ below PC

$2 \mathrm{~mm}$ lateral to midline

TARGET CORRECTED OFF ATLAS : 6 x $1.35=8.1$ below PC

TARGET ON X-RAY $2 \times 1.35=2.7$ lateral to midline

: $8.1 \times 1.1=8.9$ below PC

2.7 lateral to midline

TARGET RELATIVE TO POSTERIOR PIN ON X-RAY:

$\mathrm{AP}=2.5 \mathrm{~mm}$ Posterior

Vert $=1.5 \mathrm{~mm}$ Superior

ACTUAL TARGET RELATIVE TO POSTERIOR PIN:

$\mathrm{AP}=2.5 / 1.1=2.3 \mathrm{~mm}$ POSTERIOR

Vert $=1.5 / 1.1=1.4 \mathrm{~mm}$ SUPERIOR

LEFT CO-ORDINATES

AP

$74.9-2.3=\underline{\mathbf{7 2 . 6}}$

Lat

$58.0-2.7=\underline{\mathbf{5 5 . 3}}$

$58.0+2.7=\underline{60.7}$

Vert

$23.8+1.4=\underline{\mathbf{2 5 . 2}}$ 
APPENDIX 2 
Calculation of Co-Ordinates of Right PPN lesion in Monkey A.

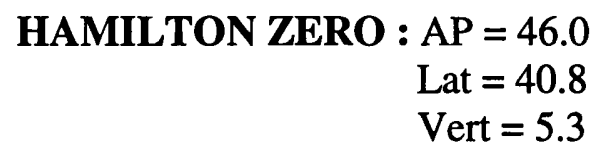

MAGNIFICATION : $5 \mathrm{~mm}$ on graph $=6 \mathrm{~mm}$ on X-Ray scale $6 / 5$ magnification $=1.2$

X-RAY AC-PC DISTANCE [cm] $: 1.5$

ACTUAL AC-PC DISTANCE[cm] $: 1.5 / 1.2=1.25$

ENLARGEMENT FACTOR OFF ATLAS $: 1.25$

TARGET FROM ATLAS : $6 \mathrm{~mm}$ below PC / $2 \mathrm{~mm}$ lateral to midline

TARGET CORRECTED OFF ATLAS : $6 \times 1.25=7.5$ below PC

$2 \times 1.25=2.5$ lateral to midline

TARGET ON X-RAY ( x Magnification):7.5 x $1.2=9.0$ below PC

2.5 lateral to midline

TARGET RELATIVE TO ZERO ON X-RAY : AP $=4.0$ Anterior to zero

Vert $=4.0$ Inferior to zero

ACTUAL TARGET RELATIVE TO ZERO : AP $=4.0 / 1.2$

3.33 Anterior to zero

Vert $=4.0 / 1.2$

3.33 Inferior to

\section{RIGHT CO-ORDINATES}

AP

$46.0-3.3=\underline{42.7}$

Lat

$40.8+2.5=\underline{43.3}$

Vert

$$
5.3-3.3=\underline{2}
$$


Calculation of Co-Ordinates of Left PPN lesion in Monkey A.

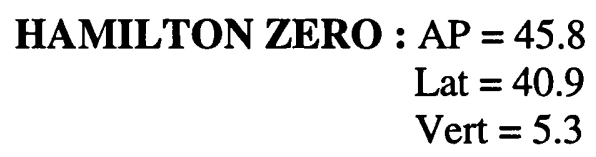

MAGNIFICATION : $5 \mathrm{~mm}$ on graph $=6 \mathrm{~mm}$ on X-Ray scale $6 / 5$ magnification $=1.2$

X-RAY AC-PC DISTANCE [cm] $: 1.5$

ACTUAL AC-PC DISTANCE[cm] $: 1.5 / 1.2=1.25$

ENLARGEMENT FACTOR OFF ATLAS $: 1.25$

TARGET FROM ATLAS : $6 \mathrm{~mm}$ below PC $/ 2 \mathrm{~mm}$ lateral to midline

TARGET CORRECTED OFF ATLAS : $6 \times 1.25=7.5$ below PC

$2 \times 1.25=2.5$ lateral to midline

TARGET ON X-RAY ( x Magnification):7.5 x $1.2=9.0$ below PC

2.5 lateral to midline

TARGET RELATIVE TO ZERO ON X-RAY $:$ AP $=6.0$ Anterior to zero

Vert $=4.0$ Superior to zero

ACTUAL TARGET RELATIVE TO ZERO : AP $=6.0 / 1.2$

5.0 Anterior to zero

Vert $=4.0 / 1.2$

3.3 Superior to zero

\section{LEFT CO-ORDINATES}

AP $\quad 45.8-5.0=\underline{\mathbf{4 0 . 8}}$

Lat

$40.9-2.5=\underline{\mathbf{3 8 . 4}}$

Vert

$5.3+3.3=\underline{8.6}$ 
Calculation of Co-Ordinates of Left PPN lesion in Monkey B.

HAMILTON ZERO : AP $=45.4$

Lat $=41.0$

Vert $=5.3$

MAGNIFICATION : $5 \mathrm{~mm}$ on graph $=6 \mathrm{~mm}$ on X-Ray scale

$6 / 5$ magnification $=1.2$

X-RAY AC-PC DISTANCE [cm] : 1.5

ACTUAL AC-PC DISTANCE[cm] $: 1.5 / 1.2=1.25$

ENLARGEMENT FACTOR OFF ATLAS $: 1.25$

TARGET FROM ATLAS : $6 \mathrm{~mm}$ below PC $/ 2 \mathrm{~mm}$ lateral to midline

TARGET CORRECTED OFF ATLAS : $6 \times 1.25=7.5$ below PC

$2 \times 1.25=2.5$ lateral to midline

TARGET ON X-RAY ( x Magnification):7.5 x $1.2=9.0$ below PC

2.5 lateral to midline

TARGET RELATIVE TO ZERO ON X-RAY : AP $=6.0$ Anterior to zero

Vert $=4.0$ Superior to zero

ACTUAL TARGET RELATIVE TO ZERO : AP $=6.0 / 1.2$

5.0 Anterior to zero

Vert $=4.0 / 1.2$

3.33 Superior to zero

\section{LEFT CO-ORDINATES}

AP $\quad 45.4-5.0=\underline{\mathbf{4 0 . 4}}$

Lat

$41.0-2.5=\underline{\mathbf{3 8 . 5}}$

Vert

$5.3+3.3=\underline{8.6}$ 
Calculation of Co-Ordinates of Right PPN lesion in Monkey B.

$$
\begin{array}{r}
\text { HAMILTON ZERO : AP }=45.8 \\
\text { Lat }=40.9 \\
\text { Vert }=5.3
\end{array}
$$

MAGNIFICATION : $5 \mathrm{~mm}$ on graph $=6 \mathrm{~mm}$ on X-Ray scale $6 / 5$ magnification $=1.2$

X-RAY AC-PC DISTANCE [cm] : 1.5

ACTUAL AC-PC DISTANCE[cm] $\quad: 1.5 / 1.2=1.25$

ENLARGEMENT FACTOR OFF ATLAS $: 1.25$

TARGET FROM ATLAS : $6 \mathrm{~mm}$ below PC / $2 \mathrm{~mm}$ lateral to midline

TARGET CORRECTED OFF ATLAS : $6 \times 1.25=7.5$ below PC

$2 \times 1.25=2.5$ lateral to midline

TARGET ON X-RAY ( x Magnification):7.5 x $1.2=9.0$ below PC

2.5 lateral to midline

TARGET RELATIVE TO ZERO ON X-RAY : AP $=4.0$ Anterior to zero

Vert $=4.0$ Inferior to zero

ACTUAL TARGET RELATIVE TO ZERO : AP $=4.0 / 1.2$

3.3 Anterior to zero

Vert $=4.0 / 1.2$

3.3 Inferior to zero

\section{RIGHT CO-ORDINATES}

AP $\quad 45.8-3.3=\underline{\mathbf{4 2 . 5}}$

Lat

$40.9+2.5=\underline{43.4}$

Vert

$5.3-3.3=\underline{\mathbf{2 . 0}}$ 
Calculation of Co-Ordinates of Left PPN lesion in Monkey C.

HAMILTON ZERO : AP $=45.0$

Lat $=49.9$

Vert $=19.5$

MAGNIFICATION : $5 \mathrm{~mm}$ on graph $=6 \mathrm{~mm}$ on X-Ray scale

$6 / 5$ magnification $=1.2$

X-RAY AC-PC DISTANCE [cm] :1.2

ACTUAL AC-PC DISTANCE[cm] $\quad: 1.2 / 1.2=1$

ENLARGEMENT FACTOR OFF ATLAS $: 1$

TARGET FROM ATLAS : $6 \mathrm{~mm}$ below PC / $2 \mathrm{~mm}$ lateral to midline TARGET CORRECTED OFF ATLAS : 6 below PC

2 lateral to midline

TARGET ON X-RAY ( $\mathrm{x}$ Magnification): 6.0 x $1.2=7.2$ below PC

2 lateral to midline

TARGET RELATIVE TO ZERO ON X-RAY : AP =5.0 Anterior to zero Vert $=10.0$ Superior to zero

ACTUAL TARGET RELATIVE TO ZERO : AP $=5.0 / 1.2$

4.2 Anterior to zero

Vert $=10.0 / 1.2$

8.3Superior to zero

\section{LEFT CO-ORDINATES}

AP

$45.0-4.2=\underline{40.8}$

Lat

$39.9-2.0=\underline{\mathbf{3 7 . 9}}$

Vert

$19.5+8.3=\underline{\mathbf{2 7 . 8}}$ 
Calculation of Co-Ordinates of Right PPN lesion in Monkey C.

HAMILTON ZERO : AP $=45.2$

Lat $=40.4$

Vert $=19.6$

MAGNIFICATION : $5 \mathrm{~mm}$ on graph $=6 \mathrm{~mm}$ on X-Ray scale

$6 / 5$ magnification $=1.2$

X-RAY AC-PC DISTANCE [cm] : 1.2

ACTUAL AC-PC DISTANCE[cm] $\quad: 1.2 / 1.2=1$

ENLARGEMENT FACTOR OFF ATLAS $: 1$

TARGET FROM ATLAS : $6 \mathrm{~mm}$ below PC $/ 2 \mathrm{~mm}$ lateral to midline

TARGET CORRECTED OFF ATLAS : 6 below PC

2 lateral to midline

TARGET ON X-RAY ( x Magnification): 6.0 x $1.2=7.2$ below PC

2 lateral to midline

TARGET RELATIVE TO ZERO ON X-RAY : AP $=6.0$ Anterior to zero

Vert $=7.5$ Superior to zero

ACTUAL TARGET RELATIVE TO ZERO : AP $=6.0 / 1.2$

5.0 Anterior to zero

Vert $=7.5 / 1.2$

6.3 Inferior to zero

\section{RIGHT CO-ORDINATES}

AP

$45.2-5=\underline{40.2}$

Lat

$40.5+2=\underline{42.4}$

Vert

$19.6+6.3=\underline{\mathbf{2 5 . 9}}$ 
Calculation of Co-Ordinates of Bilateral PPN lesions in Rhesus 4.

HAMILTON ZERO : AP $=45.9$

Lat $=41.2$

Vert $=6.2$

MAGNIFICATION : $5 \mathrm{~mm}$ on graph $=6 \mathrm{~mm}$ on X-Ray scale $6 / 5$ magnification $=1.2$

X-RAY AC-PC DISTANCE [cm] :1.45

ACTUAL AC-PC DISTANCE[cm] $\quad: 1.45 / 1.2=1.21$

ENLARGEMENT FACTOR OFF ATLAS $: 1.2$

TARGET FROM ATLAS : $6 \mathrm{~mm}$ below PC $/ 2 \mathrm{~mm}$ lateral to midline TARGET CORRECTED OFF ATLAS : $6 \times 1.2=7.2$ below PC $2 \times 1.2=2.4$ lateral to midline

TARGET ON X-RAY ( x Magnification):7.5 x $1.2=8.6$ below PC

2.4 lateral to midline

TARGET RELATIVE TO ZERO ON X-RAY : AP $=6.5$ Anterior to zero Vert $=5.5$ Superior to zero

ACTUAL TARGET RELATIVE TO ZERO : AP $=6.5 / 1.2$

5.4 Anterior to zero Vert $=5.5 / 1.2$

4.6 Superior to zero

RIGHT CO-ORDINATES

AP $\quad 45.9-5.4=\underline{40.5}$

Lat $\quad 41.2+2.4=\underline{\mathbf{4 3 . 6}}$

Vert $\quad 6.2+4.6=\underline{\mathbf{1 0 . 6}}$

\section{LEFT CO-ORDINATES}

$45.9-5.4=\underline{\mathbf{4 0 . 5}}$

$41.2-2.4=\underline{\mathbf{3 8 . 8}}$

$6.2+4.6=\underline{10.6}$ 
Calculation of Co-Ordinates of Bilateral PPN lesions in Monkey D.

HAMILTON ZERO : AP $=45.8$

Lat $=41.1$

Vert $=6.4$

MAGNIFICATION : $5 \mathrm{~mm}$ on graph $=6 \mathrm{~mm}$ on X-Ray scale $6 / 5$ magnification $=1.2$

X-RAY AC-PC DISTANCE [cm] $: 1.35$

ACTUAL AC-PC DISTANCE[cm] $: 1.35 / 1.2=1.1$

ENLARGEMENT FACTOR OFF ATLAS $: 1.1$

TARGET FROM ATLAS : $6 \mathrm{~mm}$ below PC $/ 2 \mathrm{~mm}$ lateral to midline TARGET CORRECTED OFF ATLAS : $6 \times 1.1=6.6$ below PC $2 \times 1.1=2.2$ lateral to midline

TARGET ON X-RAY ( x Magnification): 6.6 x $1.2=7.9$ below PC

2.2 lateral to midline

TARGET RELATIVE TO ZERO ON X-RAY : AP $=4.0$ Anterior to zero Vert $=5.5$ Superior to zero

ACTUAL TARGET RELATIVE TO ZERO : AP $=4.0 / 1.2$

$$
\begin{aligned}
& \text { 3.3 Anterior to zero } \\
& \text { Vert }=\text { 5.5/1.2 } \\
& \text { 4.6 Superior to zero }
\end{aligned}
$$

\section{RIGHT CO-ORDINATES}

AP $\quad 45.8-3.3=\underline{42.7}$

Lat $\quad 41.1+2.2=\underline{\mathbf{4 3 . 3}}$

Vert $\quad 6.4+4.6=\underline{11}$

\section{LEFT CO-ORDINATES}

$45.8-3.3=\underline{\mathbf{4 2 . 7}}$

$41.2-2.2=\underline{\mathbf{3 8 . 9}}$

$6.2+4.6=\underline{11}$ 
Calculation of Co-Ordinates of Bilateral PPN lesions in Monkey E.

HAMILTON ZERO : AP $=46.0$

Lat $=41.1$

Vert $=6.5$

MAGNIFICATION : $5 \mathrm{~mm}$ on graph $=6 \mathrm{~mm}$ on X-Ray scale $6 / 5$ magnification $=1.2$

X-RAY AC-PC DISTANCE [cm] :1.2

ACTUAL AC-PC DISTANCE[cm] $\quad: 1.2 / 1.2=1$

ENLARGEMENT FACTOR OFF ATLAS $: 1$

TARGET FROM ATLAS : $6 \mathrm{~mm}$ below PC $/ 2 \mathrm{~mm}$ lateral to midline TARGET CORRECTED OFF ATLAS : 6 below PC

2 lateral to midline

TARGET ON X-RAY ( $\mathrm{x}$ Magnification): 6 x $1.2=7.2$ below PC

2 lateral to midline

TARGET RELATIVE TO ZERO ON X-RAY : AP $=6.5$ Anterior to zero Vert $=8.0$ Superior to zero

ACTUAL TARGET RELATIVE TO ZERO : AP $=6.5 / 1.2$

5.4 Anterior to zero Vert $=8.0 / 1.2$

6.7 Superior to zero

\section{RIGHT CO-ORDINATES}

AP $\quad 46.0-5.4=\underline{41.6}$

Lat $\quad 41.0+2=\underline{43.0}$

Vert $\quad 6.5+6.7=\underline{13.2}$

\section{LEFT CO-ORDINATES}

$46.0-5.4=\underline{41.6}$

$41.0-2=\underline{\mathbf{3 9 . 0}}$

$6.5+6.7=\underline{13.2}$ 
Calculation of Co-Ordinates of Left PPN lesion in Cyno 3 .

HAMILTON ZERO : AP $=45.9$

Lat $=42.4$

Vert $=13.5$

MAGNIFICATION : $5 \mathrm{~mm}$ on graph $=6 \mathrm{~mm}$ on X-Ray scale

$6 / 5$ magnification $=1.2$

X-RAY AC-PC DISTANCE [cm] :1.4

ACTUAL AC-PC DISTANCE[cm] $\quad: 1.4 / 1.2=1.2$

ENLARGEMENT FACTOR OFF ATLAS $: 1.2$

TARGET FROM ATLAS : $6 \mathrm{~mm}$ below PC $/ 2 \mathrm{~mm}$ lateral to midline

TARGET CORRECTED OFF ATLAS : $6 \times 1.2=7.2$ below PC

$2 \times 1.2=2.4$ lateral to midline

TARGET ON X-RAY ( x Magnification):7.2 x $1.2=8.6$ below PC

2.4 lateral to midline

TARGET RELATIVE TO ZERO ON X-RAY : AP $=13.0$ Anterior to zero

Vert $=16.0$ Superior to zero

ACTUAL TARGET RELATIVE TO ZERO : AP $=13 / 1.2$

10.8 Anterior to zero

Vert $=16.0 / 1.2$

13.3 Superior to zero

\section{LEFT CO-ORDINATES}

AP

$45.9-10.8=\underline{\mathbf{3 5 . 1}}$

Lat

$42.4-2.4=\underline{\mathbf{4 0 . 0}}$

Vert

$13.5+13.3=\underline{\mathbf{2 6 . 8}}$ 


\section{APPENDIX 3}




\title{
The role of descending basal ganglia connections to the brain stem in Parkinsonian akinesia
}

\author{
T. Z. AZIZ* ${ }^{\star}$ L. DAVIES ${ }^{\dagger}$ J. STEN ${ }^{\dagger} \&$ S. FRANCE ${ }^{\dagger}$ \\ ${ }^{\star}$ Department of Neurosurgery, Radcliffe Infirmary and ${ }^{\dagger}$ Department of Physiology, University of Oxford, UK
}

\begin{abstract}
Akinesia is the most disabling symptom of Parkinson's disease. The neural mechanisms underlying it probably involve the descending projections of the basal ganglia to the brain stem as it improves after a pallidotomy or subthalamic nucleotomy but not after a thalamotomy. We describe the effects of lesioning the pedunculopontine nucleus in the normal primate in generating an akinetic syndrome. The possible clinical implications of this study are discussed.
\end{abstract}

Key words: 1-methyl-4-phenyl-1,2,3,6-tetrahydropyridine (MPTP), akinesia, Parkinson's disease, pedunculopontine nucleus.

\section{Introduction}

The last decade has seen an explosion in our understanding of the neural mechanisms underlying the symptoms of parkinsonism. This has largely been due to studies on primates treated with MPTP. It has been shown that an important effect of loss of the nigro-striatal dopaminergic projection is overactivity of the inhibitory outputs of the medial globus pallidus and substantia nigra pars reticulata, so that they depress the motor activity of the thalamic and brainstem structures which they target. ${ }^{1}$ Dopamine normally suppresses tonic activity of the putamen, so its absence leads to excessive inhibition of the lateral pallidum by the putamen. This, in turn, causes disinhibition of the subthalamic nucleus. The subthalamic nucleus then overdrives the medial pallidum to exert an excessive tonic inhibitory influence on its output targets, and it is thought to be this that hinders the subjects' movements. ${ }^{2,3}$

Most research has concentrated on the thalamic nuclei which are targetted by the ascending projections of the basal ganglia; hence, it is widely believed that parkinsonian akinesia results from the medial pallidum over-inhibiting the output of the basal ganglia thalamus to motor cortical areas such as the supplementary motor area. However, both clinical and experimental observations suggest that the descending projections of the pallidum and pars reticulata to the upper brain stem may be more important in akinesia. Thalamotomy successfully alleviates tremor, but it rarely improves akinesia; in fact, it can often make it worse. ${ }^{4}$

In contrast medial pallidotomy usually does alleviate akinesia, but has less effect on tremors. ${ }^{5}$ High frequency stimulation or lesioning of the subthalamic nucleus improves both symptoms. ${ }^{2,6,7}$ Because thalamic lesions do not influence the descending outputs of the basal ganglia, whereas pallidotomy and subthalamic stimulation do, the results of stereotactic surgery suggest that over-activity of the descending inhibitory projections of the basal ganglia which pass to the upper brainstem pedunculopontine area, ${ }^{1,8}$ rather than over-inhibition of the thalamus, is responsible for Parkinsonian akinesia. Indeed, the posterior third of the medial pallidum, which is the optimal pallidotomy target, is precisely the region which projects to the pedunculopontine nucleus (PPN). ${ }^{9}$ Parkinsonian patients show signs of this over-inhibition in the form of degeneration of the PPN postmortem. ${ }^{10}$

The pedunculopontine nucleus proper consists of large cholinergic neurons whose axons ramify widely, projecting rostrally back to the basal ganglia and prefrontal cortex, to the adjacent reticular formation and also downwards to the pontine and medullary reticulospinal systems responsible for locomotion and whole limb movements. ${ }^{11,12}$ In rats electrical or pharmacological stimulation of the PPN area increases, whereas PPN inhibition decreases, ${ }^{13-16}$ the animals' activity. 
TABLE I. Twenty-four-hour activity counts and their averages in monkeys 1-3 following unilateral lesions

\begin{tabular}{lccccrrrr}
\hline & $\begin{array}{c}\text { Average } \\
\text { Preop }\end{array}$ & $\begin{array}{c}\text { Post op } \\
\text { Day 1 }\end{array}$ & $\begin{array}{c}\text { Post op } \\
\text { Day 2 }\end{array}$ & $\begin{array}{c}\text { Post op } \\
\text { Day 3 }\end{array}$ & $\begin{array}{r}\text { Post op } \\
\text { Day 4 }\end{array}$ & $\begin{array}{r}\text { Post op } \\
\text { Day 5 }\end{array}$ & $\begin{array}{c}\text { Post op } \\
\text { Day 6 }\end{array}$ & $\begin{array}{c}\text { Post op } \\
\text { Day 7 }\end{array}$ \\
\hline Monkey 1 & 1217 & 648 & 695 & 711 & 720 & 837 & 1248 & 1625 \\
Monkey 2 & 1378 & 583 & 825 & 1116 & 1490 & 1392 & 1380 & 1445 \\
Monkey 3 & 2945 & 835 & 1542 & 2611 & 2894 & 2729 & 2554 & 2704 \\
Daily average & 1847 & $689^{\star \star}$ & $1020^{\star}$ & 1479 & 1701 & 1653 & 1727 & 1925 \\
SE & 551 & 76 & 263 & 578 & 636 & 562 & 415 & 393 \\
\hline
\end{tabular}

$\star_{t}>2.0 ; p<0.05$ comparing operative activity counts with those on second day after first lesion.

${ }^{\star \star} t>4.0 ; p<0.01$ comparing preoperative activity counts with those after lesions.

It seems likely therefore that further understanding of the role that the PPN plays in the generation of movement will help to elucidate why basal ganglia lesions may alleviate Parkinsonian akinesia, and to optimize their placement. We have therefore been studying the role of the PPN in locomotion and limb movements in primates. We have started by showing in rhesus monkeys that lesions of the PPN depress motor activity.

\section{Methods}

Six elderly ex-breeding female rhesus monkeys (average age: 16 years; weight $6.4 \mathrm{~kg}$ ) were used in this preliminary study. They were housed in standard Home Office approved cages with a fixed light dark cycle and ad lib food and drink except for the morning prior to surgery.

For 5-7 days prior to surgery the animals' movements were recorded using a Doppler activity meter mounted above their home cage. The sensitivity of the meter was set to record whole body and large movements of individual limbs, so the equipment was sensitive to both the overall activity of the animal and to the speed of whole limb movements. The facial expressions and limb movements of the monkeys were also videoed.

For surgery, the animals were initially sedated with intramuscular ketamine followed by intravenous infusions of althesin for intraoperative anaesthesia. The animals were then mounted in a stereotactic frame and surgery performed under sterile conditions. A contrast ventriculogram was performed with $0.5-1.0 \mathrm{ml}$ Niopam 350 . With reference to a standard atlas ${ }^{17}$ and proportional correction for size, a lesion was placed in the pedunculopontine nucleus, $2.0 \mathrm{~mm}$ lateral to the midline and $6.0 \mathrm{~mm}$ below the posterior commissure. In three animals a unilateral lesion was made first, using an electrode with a $1.0 \times 1.0 \mathrm{~mm}$ exposed tip by heating at $80^{\circ} \mathrm{C}$ for 60 seconds using a Radionics RFG lesion generator. A week later the PPN on the other side was lesioned. In three monkeys bilateral lesions were made during the first operation.

The animals then recovered in their home cage and their activity was monitored and videoed for at least 7 days postoperatively. The animals were then killed by means of an overdose of intravenous phenobarbitone and perfused with formol saline. The heads were then remounted in the stereotactic frame and the region of interest removed en bloc. After fixation, $50 \mu \mathrm{m}$ frozen sections were cut, and stained to assess the lesion size and placement.

\section{Results}

Within an hour the monkeys recovered fully from althesin anaesthesia. After the lesions they made many fewer spontaneous movements and those that they did make were clearly very much slower, as if they were 'moving through treacle'. Interestingly, their movements were still well coordinated, and showed no signs of cerebellar ataxia or intention tremor even though they were so much slower. However, their automatic movements, such as reflex withdrawal to noxious stimuli, were brisk and seemed unaffected by the lesions.

Normal rhesus monkeys make a large variety of facial movements including frequent 'threat' expressions-baring the teeth, abduction of the corners of the mouth and pushing the head forward. Immediately after the lesions, however, the animals' faces were strikingly immobile with fixed, impassive, expressions and they made no threat attempts at all. These features were very reminiscent of Parkinson's disease patients.

The first day after making unilateral lesions the quantity and speed of the monkeys' movements were reduced to approximately one third of their pre-operative level (Table I, Fig. 1), and their facial expressions became fixed. The reduction in their activity was highly significant $(t=3.6, p<0.01)$. However, they recovered to $55 \%$ of control values on the second day and returned to normal over the next week.

After the second lesion in the first three monkeys or bilateral lesions in the subsequent three monkeys, the poverty and slowing of the animals movements showed little improvement in the week following surgery. On the first postoperative day activity counts had dropped by $74 \%$ [Table II, Fig. 1 $(t=5.5 p<0.01)$ ], and on the seventh day they were still reduced by over $70 \%$. Also the monkeys' lack of facial expression persisted, a fact confirmed on reviewing the videotapes. 


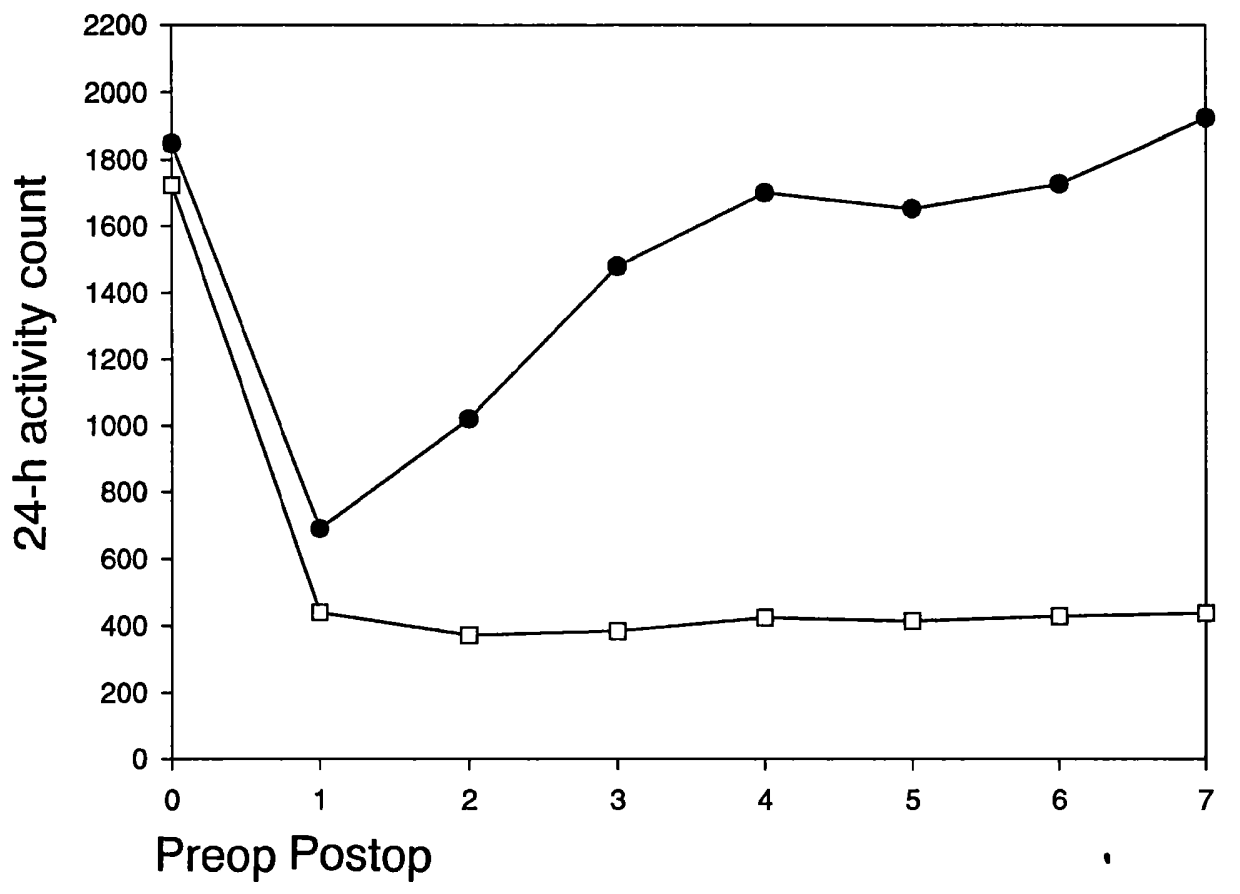

FIG. 1. Averaged activity counts in all animals following unilateral (๑) or bilateral $(\square)$ lesions in first week post operation.

Figure 2 shows the location of the lesions in one monkey. Those in the other five monkeys included the same areas. Almost certainly the cholinergic pedunculopontine nucleus was ablated on both sides in all the monkeys, together with the surrounding mesencephalic locomotor region; but the lesions left most of the red nucleus and the fibres of the superior cerebellar peduncle intact.

\section{Discussion}

We chose to make lesions of the pedunculopontine nucleus because it receives dense projections from the medial globus pallidus, and it projects in turn to the pontine and medullary reticulospinal systems. ${ }^{8}$ In rats stimulation there produces locomotor activity, whilst inactivation by GABA agonists decreases activity. ${ }^{13-16}$ Moreover, in the MPTP model of Parkinson's disease in primates GABAergic projections of the medial pallidum to the PPN are overactive, which probably explains the immobility of these animals. ${ }^{1}$ In accord with the hypothesis that the PPN is overinhibited in Parkinson's disease the PPN degenerates in akinetic Parkinson's patients. ${ }^{10}$

Clearly, our lesions greatly slowed the animals' movements and reduced their spontaneous activity. The animals recovered in only a few days after unilateral lesions. This was probably because the ascending and descending projections from each PPN to the basal ganglia and pontine and medullary reticulospinal systems are bilateral ${ }^{8}$ so the intact side could take over. However, when the surviving PPN was ablated or both were destroyed at the same time the animals' activity did not recover. These reductions in the monkey's movements therefore support our hypothesis that Parkinsonian bradykinesia results from overinhibition of the PPN by an overactive medial pallidum. Dyskinesias are alleviated by a VOP thalamotomy and bilaterally by a medial pallidotomy (though the ipsilateral effect is less dramatic). Both suggest that dyskinesias are a pallidothalamo-cortical loop phenomenon. Lesioning the medial pallidum alleviates the bradykinesia/akinesia of parkinsonism. It is interesting that bilateral pallidotomies are far more effective than unilateral lesions in this respect. ${ }^{18,19}$ This would suggest that although the ascending and descending projections of the PPN are bilateral, both need to be released from pallidal inhibition for movement to be restored.

The large lesions which we made probably included not only the cholinergic pedunculopontine nucleus, but also adjacent areas of the mesencephalic locomotor region. However, it is unlikely that they had a significant effect on cerebellar outflow because staining with Luxol Fast Blue which accentuates fibres, confirmed that the majority of the axons in the the superior cerebellar peduncle remained intact. Moreover after the lesions it was remarkable how well coordinated and tremor-free the animals' movements remained, despite their rarity and slowness.

Another possibility is that the animals' inactivity was non-specific and nothing to do with the descending pallidal projections. Our lesions might have affected the general reticular activating system, but if they had destroyed a substantial proportion of that system we might have expected the animals to have slept, and not to have moved at all, rather than simply moving much more slowly and less often. 
TABLE II. Twenty-four-hour activity counts, and their averages, in monkeys $1-3$ following a second lesion and in monkeys 4-6 following bilateral lesions

\begin{tabular}{lcccccccc}
\hline & $\begin{array}{c}\text { Average } \\
\text { Preop }\end{array}$ & $\begin{array}{c}\text { Post op } \\
\text { Day 1 }\end{array}$ & $\begin{array}{c}\text { Post op } \\
\text { Day 2 }\end{array}$ & $\begin{array}{c}\text { Post op } \\
\text { Day 3 }\end{array}$ & $\begin{array}{c}\text { Post op } \\
\text { Day 4 }\end{array}$ & $\begin{array}{c}\text { Post op } \\
\text { Day 5 }\end{array}$ & $\begin{array}{c}\text { Post op } \\
\text { Day 6 }\end{array}$ & $\begin{array}{c}\text { Post op } \\
\text { Day 7 }\end{array}$ \\
\hline Monkey 1 & 1515 & 708 & 720 & 718 & 726 & 644 & 704 & 754 \\
Monkey 2 & 1609 & 211 & 163 & 218 & 326 & 437 & 355 & 441 \\
Monkey 3 & 2825 & 638 & 437 & 325 & 339 & 256 & 281 & 254 \\
Monkey 4 & 2160 & 121 & 150 & 256 & 420 & 445 & 455 & 420 \\
Monkey 5 & 1102 & 451 & 320 & 287 & 278 & 301 & 324 & 299 \\
Monkey 6 & 1234 & 508 & 435 & 501 & 455 & 402 & 450 & 461 \\
Daily average & 1724 & $440^{\star \star}$ & $371^{\star \star}$ & $384^{\star \star}$ & $424^{\star \star}$ & $414^{\star \star}$ & $428^{\star \star}$ & $438^{\star \star}$ \\
SE & 270 & 95 & 87 & 78 & 66 & 56 & 62 & 72 \\
\hline
\end{tabular}

${ }^{\star} t>2.0 ; p<0.05$ comparing preoperative activity counts with those on second day after first lesion.

${ }^{\star} t>4.0 ; p<0.01$ comparing preoperative activity counts with those after lesions.

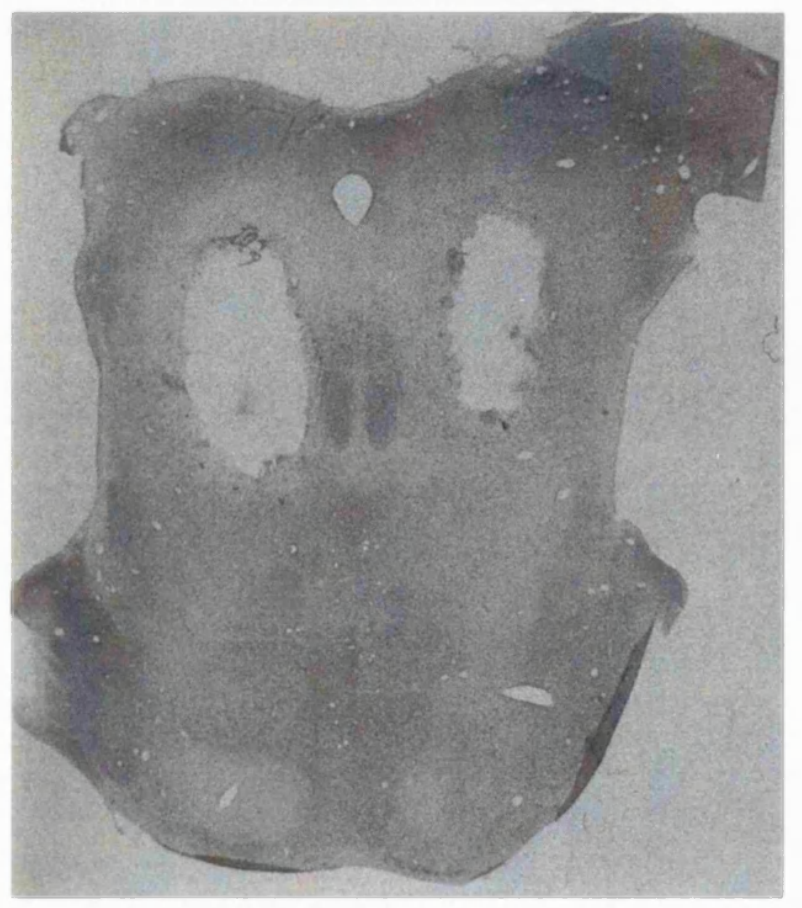

FIG. 2. Histological site of lesions in one monkey.

The Doppler activity meter was sensitive both to the speed of the animals' movements and to their over all level of activity; hence, the reduction in counts confirmed our video observation that the animals moved more slowly after the lesions.

It might also be argued that the lesions affected the animals' motivation to move rather than their ability to do so. Where motivation can be said to end and execution to begin is largely a question of semantics. However, if our lesions had eliminated the animals' motivation altogether, again we would have expected them not to move at all. Instead, their spontaneous movements were slowed; but their reflex movements were relatively intact. Therefore, it seems likely that the lesions had the specific effects of reducing the number and slowing down the performance of their voluntary movements, without affecting their general arousal or motivation; in short rendering them akinetic as in Parkinson's disease.
Understanding the nature of akinesia has implications beyond that of parkinsonism. Akinesia is a feature of parkinson-like syndromes such as multisystem atrophy and progressive supranuclear palsy, a condition in which there is known to be a loss of pedunculopontine neurons, ${ }^{20}$ which resist medical therapy. If the brain stem mechanisms described are central to akinesia it remains a possibility that stimulators implanted in the upper brain stem, or even cholinergic cellular implants, may be clinically beneficial.

\section{Acknowledgements}

This work is supported by the Medical Research Council. We thank Mr Jonathan Winters for his invaluable technical support.

\section{References}

1 Sambrook MA, Crossman AR, Mitchell I, Robertson RG, Clarke CE, Boyce S. The basal ganglia mechanisms mediating primate models of movement disorders. In: Crossman AR, Sambrook MA (eds) Neural Mechanisms in Disorders of Movement London: John Libbey 1989:123-44.

2 Aziz TZ, Peggs D, Agarwal E, Sambrook MA, Crossman AR. Subthalamic nucleotomy alleviates parkinsonism in the MPTP exposed primate. Br $\mathcal{F}$ Neurosurg 1992;6:575-82.

3 Bergman $\mathrm{H}$, Wichmann T, DeLong MR. Reversal of experimental parkinsonism by lesions of the subthalamic nucleus. Science 1990;249:1436-8.

4 Laitinen L, Vilkki J. Measurement of parkinsonian hypokinesia with Purdue Pegboard and Motor reaction time tests. In: Siegfried J (ed.) Parkinsons Disease. Berlin: Huber 1973;2:185-92.

5 Baron MS, Vitek JL, Bakay RAE, DeLong MR et al. Treatment of advanced Parkinson's disease by posterior GPi pallidotomy: 1-year results of a pilot study. Ann Neurol, 1996;40:355-66.

6 Benazzouz A, Boraud T, Feger J, Burbaud P, Bioulac, Gross C. Alleviation of experimental hemiparkinsonism by high frequency stimulation of the subthalamic nucleus in primates, a comparison with L-dopa treatment. Mov Disord 1996;11(6):627-32.

7 Limousin P, Pollak P, Benazzouz et al., Effect on parkinsonian signs and symptoms of bilateral subthalamic nucleus stimulation. Lancet 1995;345:91-5. 
8 Jackson A, Crossman AR. Nucleus tegmenti pedunculopontinus, efferent connections with special reference to the basal ganglia. Neuroscience 1983;10:725-65.

9 Takada $M$. Descending pathways of the basal ganglia. Kaibogaku Zanshi 1995;70(4):289-93.

10 Zweig RM, Jankel WR, Hedreen JC. The pedunculopontine nucleus in Parkinsons disease. Ann Neurol 1989;26:41-6.

11 Garcia-Rill E. The pedunculopontine nucleus. Prog Neurobiol 1991;36:363-89.

12 Steriade $M$, et al. Projections of cholinergic and noncholinergic neurones of the brain stem core to relay and associational thalamic nuclei in the cat and macaque monkey. Neuroscience 1988;25:47-67.

13 Brudzynski SM, Houghton PE, et al. Involvement of neuronal cell bodies in the mesencephalic locomotor region in the initiation of locomotor activity of freely moving rats. Brain Res Bull 1986;16:377-81.

14 Milner KL, Mogenson GJ. Electrical and chemical activation of the mesencephalic and subthalamic locomotor regions in freely moving rats. Brain Res 1988;452:273-85.
15 Garcia-Rill E, Houser CR, et al. Locomotion inducing sites in the vicinity of the pedunculopontine nucleus. Brain Res Bull 1987;18:731-8.

16 Mogenson GJ, Wu M. Differential effects on locomotor activity of injections of procaine into mediodorsal talamus and pedunculopontine nucleus. Brain Res Bull 1988;20:241-6.

17 Shanta TR, Manocha SL. A stereotactic atlas of the fava monkey brain. New York: Karger, 1968.

18 Johannson F, Malm J, et al. Usefulness of pallidotomy in advanced Parkinson's disease. $\mathcal{F}$ Neurol Neurosurg Psychiatry 1997;62:125-32.

19 Scott R, Gregory R, et al. Neuropsychological, neurological and functional outcome following pallidotomy for Parkinson's disease: a consecutive series of 8 simultaneous bilateral and 12 unilateral procedures. Brain 1998;121:659-75.

20 Zweig RM, Whitehouse PJ, Casanova MF, Walker LC, Jankel WR, Price DL. Loss of pedunculopontine neurons in progressive supranuclear palsy. Annl Neurol $1987 ; 22(1): 18-25$. 


\title{
The pallidotomy debate
}

\author{
C. B. CARROLL ${ }^{1}$, R. SCOTT ${ }^{2}$, L. E. DAVIES S $^{3}$ T. AZIZ ${ }^{1}$ \\ Department of ${ }^{1}$ Neurosurgery, ${ }^{2}$ Neuropsychology and ${ }^{3}$ Neurophysiology, Radcliffe Infirmary, Woodstock Road, Oxford, UK
}

\begin{abstract}
It has been suggested that image-guidance and macro-stimulation alone are not sufficiently accurate to result in safe and effective lesion localization in pallidotomy for Parkinson's disease when compared with micro-electrode recording. This review analyses the data in the series published to date, and compares the safety and efficacy of the two techniques, finding no evidence to support this claim. In addition, evidence regarding the necessary accuracy of lesion placement is reviewed.
\end{abstract}

Key words: Micro-electrode recording, pallidotomy, Parkinson's disease.

\section{Introduction}

In an editorial published earlier this year ${ }^{1}$ regarding pallidotomy for Parkinson's disease, Obeso et al. wrote:

A proportion of functional neurosurgeons still think that stimulating with the lesioning probe is enough to avoid complications and ensure positive clinical results. We oppose such a view.

It must be admitted that at present definitive evidence that microrecording is essential for optimal basal ganglia surgery is lacking. However, anecdotal information from centres using less rigorous assessment methods should be viewed with extreme caution.

These two quotations illustrate the nature of the pallidotomy debate, which is essentially whether or not micro-electrode recording is necessary for safe and effective lesion location in pallidotomy for Parkinson's disease. Although this may seem to be a technical point of limited relevance, the debate regarding this issue is becoming more heated. It has been estimated that in the United States 50,000 new cases of Parkinson's disease are diagnosed every year, with the number of Americans affected being in the order of 1 million. ${ }^{2}$ In the majority of cases, medical therapy will begin to fail within 5-10 years ${ }^{3}$ and pallidotomy is likely to become an alternative option. Medicare has only recently agreed to cover the cost of pallidotomy for Parkinson's disease, a procedure which was previously regarded as experimental. Accurate lesion placement is intuitively an important aspect of the procedure, and proponents of micro-electrode recording suggest that this is the only technique accurate enough to ensure good placement. If this claim is found to be substantiated, then the ability to perform pallidotomies will be limited to those centres with micro-electrode recording facilities, which are expensive in terms of both financial and personnel resources. With pallidotomies costing up to $\$ 40,000$, the stakes for both neurosurgeons and patients are high.

Neurosurgical intervention for Parkinson's disease has a long history. ${ }^{4-7}$ Thalamotomy was, and to a large extent still is, considered to be the best surgical treatment for drug-resistant tremor in Parkinson's disease. However, pallidotomy, by alleviating rigidity and akinesia which are more disabling to patients in functional terms, has the ability to more dramatically improve a patient's quality of life. ${ }^{7,8}$ Despite a recent surge of interest in the procedure and extensive review, the reasons for the efficacy of the operation without apparent motor deficit remain unclear. ${ }^{9-13}$ PET studies tend to support the theory that lesioning the posteroventral portion of the internal segment of the globus pallidus (Gpi) diminishes the pathologically increased inhibitory outflow from the Gpi to the thalamus and the pedunculopontine nucleus, ${ }^{14}$ thus facilitating movement. ${ }^{15-17}$

Image-guided pallidotomy involves the use of CT and MRI, with or without ventriculography, in conjunction with a stereotactic atlas to determine the co-ordinates of the target. ${ }^{11,18}$ Various techniques are employed to allow for variation in brain size between individuals. The final location of the lesion 
Table I. Peri- and postoperative complications associated with pallidotomy for Parkinson's disease

\begin{tabular}{lcc}
\hline & $\begin{array}{c}\text { Image-guided (\%) } \\
\text { (Refs 18,21,22,24,31,32,34,35) }\end{array}$ & $\begin{array}{c}\text { MER-guided (\%) } \\
\text { (Refs 1,20,23,25,33) }\end{array}$ \\
\hline Overall complication rate & 9.8 & 17.4 \\
Intra-cerebral haemorrhage & 0.6 & 7.0 \\
Visual field cut & 4.6 & 1.5 \\
Permanent hemiparesis & 0.8 & 0.5 \\
\hline
\end{tabular}

is determined with the aid of test lesions and macrostimulation with the lesioning electrode, to avoid damage to the optic tract or internal capsule. The technique obviously relies on the resolution of the scans and the accuracy of the atlas. The total time required for the operation (for the patient) is in the region of 3-4h.

Micro-electrode recording involves the definition of an initial target by MRI and revision of the target based on the definition of neurophysiological landmarks determined by advancing micro-electrodes along recording tracts. Usually 3-8 recording tracts are made before the lesioning electrode is inserted. Macro-stimulation with the lesioning electrode and test lesions are then employed to ensure safe and effective lesion placement. The whole procedure (for the patient) can take up to $12 \mathrm{~h}$.

\section{Image-guided versus micro-electrode-guided target localization}

The issues surrounding pallidotomy can broadly be divided into two groups: those of safety and efficacy. Into the 'safety' group fall considerations such as intraoperative haemorrhage or seizure, and rates of peri- or post operative complications, including the neuropsychological consequences of 'misplaced' lesions, which largely remain unknown. ${ }^{2}$ These are summarized for both techniques in Table I. Considerations of 'efficacy' include measurement of clinical outcome as determined by a variety of outcome measures and length of duration of positive response. The issue of accurate lesion location is regarded as central to both safety and efficacy, lesions which are too medial endangering the internal capsule, those which are too ventral endangering the optic tract, and those which are too lateral, anterior or dorsal being ineffective.

Simply stated, supporters of micro-electrode recording contend that image-guidance alone is not sufficiently accurate to determine the borders of the Gpi, and is therefore more likely to result in lesions affecting the internal capsule or optic tract, resulting in less well-placed lesions that are therefore less effective $^{19}$ (M. R. DeLong, personal communication). Conversely, supporters of image-guided lesion placement suggest that the technique of micro-electrode recording carries with it a higher intra-operative risk and complication rate, as well as being more demanding in terms of time and resources, and therefore less practical.

Despite the large numbers of patients who have undergone pallidotomy, reports in the peer-reviewed literature are limited. Comparison of the two techniques is therefore made difficult by the low numbers of patients involved. It has been suggested that a registry or data centre be developed to monitor the progress of the procedure in different patient groups and the use of different techniques. ${ }^{6}$

The questions that this review addresses are:

1. Does the use of micro-electrode recording result in safer lesions?

2. Does the use of micro-electrode recording result in more effective lesions?

3. Does the use of micro-electrode recording result in more accurately placed lesions?

4. How accurately placed do lesions need to be?

\section{Safety of lesions}

Avoidance of the optic tract and internal capsule remains central to the safety of the procedure. In both techniques, the proximity of both structures is assessed by determining the threshold for a response to stimulation with the lesioning electrode-the threshold being assumed to be inversely proportional to the distance. However, the use of micro-electrode stimulation and recording of visualevoked responses is thought to ensure an extra element of safety. Critics of the technique point out that determination of a stimulation response requires an alert and co-operative patient. As patients undergoing pallidotomy are off their medication and easily tired it is not surprising that discrepancies between visual-evoked responses and stimulation data have been reported. ${ }^{20}$

Analysis of the data reviewed reveals there to be 480 patients in the group with image-guided lesions, and 201 patients with micro-electrode-guided lesions. Comparison of the complication rates of the two techniques is hindered by the qualitative rather than quantitative reporting of some of the complications, and by the small size of some of the studies. Furthermore, authors do not report complications that they have not looked for, such as the neuropsychological consequences of apparently well-placed 
lesions. ${ }^{18}$ Also, combining the data from all the studies will inevitably result in some duplication. However, the use of image-guidance alone does seem to result in a higher incidence of damage to the optic tract and internal capsule (Table I), although the overall complication rate is higher using micro-electrode recording than with imageguidance alone (17.4 versus $9.8 \%$, respectively). This is largely attributable to the increased risk (10 times) of intraoperative haemorrhage with microelectrode recording (Table I). However, studies reporting high complication rates ${ }^{1,21-23}$ are preliminary series or pilot studies, and attribute their high complication rate to inexperience. Indeed, later reports from the same authors seem to confirm improvement. ${ }^{24}$ The safety of pallidotomy, as with many other surgical interventions, is therefore likely to depend more on the experience of the surgeon than on the technique employed. However, future reports with greater numbers of patients are necessary to show definitively that the difference in complication rate demonstrated here is a reporting bias of the series published to date.

\section{Efficacy of lesions}

The issue of efficacy is harder to resolve than that of safety. Parkinson's disease is a progressive condition, the underlying rate of which is not altered, it is generally thought, by the purely symptomatic intervention of pallidotomy. (However, one study does suggest that pallidotomy slows the rate of progression of the disease). ${ }^{25}$ In addition, the disease of each patient progresses at a different rate. These considerations make long-term evaluation of pallidotomy difficult. Perhaps a more important consideration in the assessment of efficacy is the placebo response. This has been documented to be as high as $30 \%{ }^{26}$ and last for up to 2 years. The group of patients undergoing pallidotomy are highly motivated and receive intensive monitoring and follow-up. It would be unethical to subject the procedure to randomized trial with sham operation as a control, and it will therefore never be possible to control for the placebo effect of having undergone surgery. The results of studies showing improvement of up to $30 \%$ with limited follow-up therefore need to be interpreted in this context.

In terms of lesion location, a few studies have demonstrated that lesions placed more posteriorly and ventrally in the GPi are associated with a better clinical outcome. ${ }^{27,28}$ It has been suggested that micro-electrode recording allows identification of this 'sensorimotor' portion of the Gpi, thus facilitating placement of a more effective lesion. ${ }^{19}$ Microelectrode recording investigations have also led to the determination of the somatotopic organization of the Gpi, which might further improve target localization. ${ }^{16}$ However, it is also the case that the efficacy of a proposed lesion site can be assessed intraoperatively by the formation of a 'test lesion' with the lesioning electrode, although it is clear that some of the potential beneficial effects of the lesion (such as reduction in freezing, gait disturbance and dyskinesias) cannot be assessed intraoperatively in this way. Nevertheless, the use of macrostimulation and the production of 'test' lesions may be sufficient to result in effective lesion placement without the use of micro-electrode recording.

Comparison of the studies reviewed here is made impossible by the absence of a standardized protocol for assessment. Further difficulties include some studies only reporting outcomes for the contralateral limb, not differentiating between 'on' and 'off' states, quoting mean values instead of the median in non-parametric data or failing to quantify any observed benefit. However, rough comparison of the efficacy of the two surgical techniques does not demonstrate any marked differences between the two. Which objective measure most accurately reflects change in the patients' functional capacity and quality of life (and would therefore be the most valid) is the subject of further debate ${ }^{26}$ and is currently under review.

\section{Accuracy of lesion placement}

It has yet to be demonstrated that micro-electrode recording techniques result in more accurate placement of lesions than image-guidance and macrostimulation alone. Indeed, problems such as brain sag and CSF leakage with micro-electrode recording increase the value of macrostimulation in determination of lesion location. A number of studies have investigated the accuracy with which lesions can be placed using $\mathrm{CT}^{29}$ or MRI guidance. ${ }^{30}$ One study showed that the use of MRI for target localization was able to result in the placement of lesions that were 'exactly as chosen' in the rostrocaudal and mediolateral planes with a discrepancy of $1.7 \mathrm{~mm}$ in the dorsolventral plane. ${ }^{30}$ The authors concluded that with the appropriate precautions taken to minimise the distortion of the MRI image it would be possible to locate the target to within $1 \mathrm{~mm}$. It may be the case that the degree of accuracy of lesion placement using image guidance is dependent on the type of system used. In Oxford the technique involves the fusion of a CT image with an MRI image and a computerized stereotactic atlas. ${ }^{36}$ This allows for correction for variation in brain size in three dimensions. This may result in more accurate target location with image-guidance than that reported by Lozano et al. ${ }^{20}$ who corrected only to the intercommissural line, the correction factor of which is different from that in the lateral plane.

In order for the question regarding lesion location to be resolved, accurate postoperative mapping of the lesion must be carried out. Postoperative analysis of lesion location is fraught with problems: CT does not provide images with sufficient resolution; 
the images produced by MRI scanners are anatomically distorted. The resolution of any image analysis system will only be as good as the scan which it analyses and with scanners in common use this is usually in the order of $1 \mathrm{~mm}$ at best. The actual process of lesion measurement (positioning a marker on a screen) introduces another factor of error. Finally, mapping the lesion to an anatomical location depends on fusing the image of the lesion with a standardized atlas, again introducing error. For final lesion location to be determined objectively, postmortem examination with retrospective correlation with clinical outcome needs to be carried out.

\section{What degree of accuracy is necessary?}

Although the general target area for pallidotomy has been defined, the necessary degree of accuracy of lesion location has not been determined (indeed it has been suggested that the margin of error may be as large as $2-3 \mathrm{~mm}$ ). ${ }^{2}$ Early studies have found there to be no correlation between clinical outcome and size and site of the lesion. ${ }^{29}$ Interestingly, PET studies have provided evidence that the degree of postoperative functional improvement correlates most strongly with preoperative lentiform nucleus activity. ${ }^{17}$ Indeed, the authors state that their findings "support observations that subtle variations in lesion size and location do not impact greatly on clinical outcome". Again, until postmortem data become available to allow accurate determination of lesion location and correlation of location with clinical outcome, the question as to the necessary degree of accuracy will remain unanswered.

\section{Further considerations}

As pallidotomy becomes more widely used, there will be increasing need for the proper evaluation of the feasibility and safety of bilateral pallidotomies, whether contemporaneous or staged. One study found no evidence of an increased risk of complications with contemporaneous pallidotomy, ${ }^{31}$ although another found that contemporaneous pallidotomy resulted in decreased articulation rates. ${ }^{18}$ Additional concerns are of increasing hypersalivation and dysarthria following bilateral pallidotomy (T. Aziz, personal communication; M. R. DeLong, personal communication). The benefits of contemporaneous pallidotomy for the patient are obvious. However, it should be noted that the time required for micro-electrode recording makes contemporaneous bilateral operations using this technique impractical.

\section{Conclusions}

The evidence currently available suggests that micro-electrode-guided pallidotomy results in a greater peri-operative complication rate than that associated with image-guidance alone. There is insufficient evidence to date to compare objectively efficacy of the two techniques; longer-term followup with a standardized assessment protocol is needed to finally resolve this issue. In addition, there is no evidence that micro-electrode recording results in more accurate lesion placement, nor indeed that more than $1 \mathrm{~mm}$ accuracy is necessary for effective lesion placement. Furthermore, micro-electrode recording is undoubtedly more demanding in terms of time and personnel resources, and is therefore less practical in the setting of heavy clinical demand. As health-care providers, we aim to provide high quality care within the reasonable restrictions of a health service budget, and therefore currently see no reason to advocate the use of micro-electrode recording in pallidotomy for Parkinson's disease.

\section{References}

1 Obeso JA, Guridi J, DeLong M. Surgery for Parkinson's disease. $\mathcal{f}$ Neurol Neurosurg Psychiatry 1997;62:2-8.

2 Kelly PJ. Pallidotomy in Parkinson's disease. Neurosurgery 1995;36:1154-7.

3 Curtis L, Lees AJ, et al. Effect of L-Dopa on the course of Parkinson's disease. Lancet 1984;July:21112.

4 Selby G. Stereotactic surgery for the relief of Parkinson's disease. Part 1. A critical review. $\mathcal{F}$ Neurol Sci 1967;5:315-42.

5 Selby G. Stereotactic surgery for the relief of Parkinson's disease. Part 2. An analysis of the results in a series of 303 patients (413 operations). $\mathcal{F}$ Neurol Sci 1967;5:343-75.

6 Goetz CG, Diederich NJ. There is a renaissance of interest in pallidotomy for Parkinson's disease. Nature Med 1996;2:510-14.

7 Olanow CW. GPi pallidotomy-have we have made a dent in Parkinson's disease? Ann Neurol 1996; 40: 341-3.

8 Iacono RP, Lonser RR Reversal of Parkinson's akinesia by pallidotomy. Lancet 1994:343:418-19.

9 Marsden CD, Obeso JA. The functions of the basal ganglia and the paradox of stereotactic surgery in Parkinson's disease. Brain 1994;117:877-97.

10 Siegfried J, Lippitz B. Bilateral chronic electrostimulation of ventroposterolateral pallidum: a new therapeutic approach for alleviating all parkinsonian symptoms. Neurosurgery 1994;35:1126-30.

11 Iacono RP, Lonser RR, Mandybur G, Yamada S. Stimulation of the globus pallidus in Parkinson's disease. Br F Neurosurg 1995;9:505-10.

12 Iacono RP, Lonser RR, Oh A, Yamada S. New pathophysiology of Parkinson's disease revealed by posteroventral pallidotomy. Neurol Res 1995;17:178-80.

13 Iansek R, Bradshaw J, Phillips J, Morris ME, Cunnington R. Review article: the functions of the basal ganglia and the paradox of stereotaxic surgery in Parkinson's disease. Brain 1995; 118:1613-17.

14 Shima $F$, Iacono $R$, Sakata $S$, Fukui $M$, Kato $M$. Mechanism of posteroventral pallidotomy in Parkinson's disease. Stereotact Funct Neurosurg 1994;62:11319.

15 Ceballos-Baumann AO, Obeso JA, Vitek JL, DeLong $M R$, Bakay $R$, Linazasoro $G$, et al. Restoration of thalamocortical activity after posteroventral pallidotomy in Parkinson's disease. Lancet 1994:344:814. 
16 Dogali M, Beric A, Sterio D, Eidelberg D, Fazzini E, Takikawa S, et al. Anatomic and physiological considerations in pallidotomy for Parkinson's disease. Stereotact Funct Neurosurg 1994;62:53-60.

17 Eidelberg D, Moeller JR, Ishikawa T, Dhawan V, Spetsieris $\mathrm{P}$, Silbersweig $\mathrm{D}$, et al. Regional metabolic correlates of surgical outcome following unilateral pallidotomy for Parkinson's disease. Ann Neurol 1996;39:450-9.

18 Scott R, Gregory R, Carroll CB, Hyman N, Leather $\mathrm{C}$, Hines $\mathrm{N}$, et al. Neuropsychological, neurological and functional outcome following pallidotomy for Parkinson's disease: a consecutive series of 8 simultaneous bilateral and 12 unilateral procedures Brain 1998;121 (in press).

19 Bakay RAE, DeLong MR, Vitek JL. Posteroventral pallidotomy for Parkinson's disease. $f$ Neurosurg 1992;77:487-8.

20 Lozano A, Hutchison W, Kiss Z, Tasker R, Davis K, Dostrovsky J. Methods for microelectrode-guided posteroventral pallidotomy. $\mathcal{F}$ Neurosurg 1996;84:194-202.

21 Laitinen LV, Bergenheim AT, Hariz MI. Ventroposterior pallidotomy can abolish all parkinsonian symptoms. Stereotact Funct Neurosurg 1992:58:14-21.

22 Laitinen LV, Bergenheim AT, Hariz MI. Leksell's posteroventral pallidotomy in the treatment of Parkinson's disease. $\mathcal{F}$ Neurosurg 1992;76:53-61.

23 Baron MS, Vitek JL, Bakay RAE, Green J, Kaneoke Y, Hashimoto T, et al. Treatment of advanced Parkinson's disease by posterior GPi pallidotomy: 1-year results of a pilot study. Ann Neurol 1996;40:355-66.

24 Laitinen LV. Ventroposterior pallidotomy. Stereotact Funct Neurosurg 1994;62:41-52.

25 Dogali M, Fazzini E, Kolodny E, Eidelberg D, Sterio $\mathrm{D}$, Devinsky O, et al. Stereotactic ventral pallidotomy for Parkinson's disease. Neurology 1995;45:753-61.

26 Obeso JA, Linazasoro G, Rothwell JC, Jahanshahi M, Brown R. Assessing the effects of pallidotomy in Parkinson's disease. Lancet 1996;347:1490.
27 Svennilson E, Torvik A, Lowe R, Leksell L. Treatment of parkinsonism by stereotactic thermolesions in the pallidal region. Acta Psychiatr Scand 1960;35:35877.

28 Vitek JL, Hashimoto T, Baron M, Kaneoke Y, Turner $\mathrm{R}$, Bakay R, et al. Pallidotomy in Parkinson's disease: correlation of lesion location to clinical outcome. Mov Disord 1994;9:477.

29 Hariz MI. Correlation between clinical outcome and size and site of the lesion in computed tomography guided thalamotomy and pallidotomy. Stereotact Funct Neurosurg 1990;55:172-85.

30 Lehman RM, Mezrich R, Sage J, Goldbe L. Peri- and postoperative magnetic resonance imaging localization of pallidotomy. Stereotact Funct Neurosurg 1994;62:6170.

31 Iacono RP, Lonser RR, Mandybur G, Yamada S. Stimulation of the globus pallidus in Parkinson's disease. $\mathrm{Br} \mathcal{F}$ Neurosurg 1995;9:505-10.

32 Johannson F, Malm J, Nordh E, Hariz M. Usefulness of pallidotomy in advanced Parkinson's disease. $\mathcal{F}$ Neurol Neurosurg Psychiatry 1997;62:125-32.

33 Lozano AM, Lang AE, Galvez-Jimenez N, Miyasaki J, Duff J, Hutchinson WD, et al. Effect of Gpi pallidotomy on motor function in Parkinson's disease. Lancet 1995;346:1383-7.

34 Sutton JP, Couldwell Wm, Lew MF, Mallory L, Grafton S, DeGiorgio C, et al. Ventroposterior medial pallidotomy in patients with advanced Parkinson's disease. Neurosurgery 1995;36: 1112-17.

35 Iacono RP, Shima F, Lonser RR, Kuniyoshi S, Maeda $\mathrm{G}$, Yamada S. The results, indications and physiology of posteroventral pallidotomy for patients with Parkinson's disease. Neurosurgery 1995;36:1118-27.

36 Papanastassiou V, Rowe J. Scott R, Silbum P, Davies L, Aziz T. Use of the Radionics Image Fusion $^{\mathrm{TM}}$ and Stereoplan ${ }^{\mathrm{TM}}$ programs for target localisation in functional neurosurgery. $\mathcal{f}$ Clin Neurosci 1998;5:28-32. 


\section{Letters to the Editor}

The Editors welcome all letters; whether they are short case reports, preliminary reports of research, discussion or comments on papers published in the joumal. Letters commenting on a paper may be sent to the authors of that paper so that their reply may appear in the same issue.

There is no rigid limit to length, but in general the shorter the letter the better the chance of publication. Authors should follow the same guidelines given for the preparation and submission of articles on the inside back cover of each issue, especially that regarding tables or illustrations. Letters should be signed by all authors.

\section{Operative treatment of cervical spondylosis}

SIR-Whilst welcoming Marks' recent editorial on operative treatment of cervical spondylosis, ${ }^{1}$ we think he misses two fundamental issues. The first is to define which patients benefit from operation and, inseparable from this, how should we measure outcome in an unbiased and validated way? It is only after establishing this we can start to ask which operation benefits patients most (e.g. fusion vs discectomy).

In a recent review of outcome measures for cervical spine surgery, Thomas concluded that none were satisfactory. ${ }^{2}$ Forty years after the introduction of Clowards procedure ${ }^{3}$ we still rely on retrospective outcome assessment by the surgeon who performed the procedure, with its inherent bias, to guide us in patient management. Radiological evidence of fusion has also been employed as a useful outcome measure. ${ }^{4}$ However, prospective studies have shown that post-operative radiological appearances in terms of vertebral alignment do not necessarily correlate with functional outcome. ${ }^{5}$ Objective assessment of pain, disability and quality of life can all be achieved cheaply through a postal and telephone questionnaire which we and others are currently validating.

To understand which patient benefits from operation we have to know the natural history of symptomatic cervical spondylosis. This is uncertain because currently a varying proportion of patients are treated surgically. However, the idea that the untreated individuals will necessarily develop progressive disability is not supported by reliable evidence. ${ }^{6-9}$ In addition, the disease may remain static for lengthy periods or even improve without treatment. ${ }^{6,10}$ The widespread belief that patients with radicular symptoms will eventually develop overt myelopathy is also not based on epidemiological evidence. ${ }^{11}$

It is likely that cervical spondylotic radiculomyelopathy (CSRM) is a heterogeneous condition in which there may be subgroups of patients who will benefit from surgery. Many factors have been implicated in the propensity to develop CSRM, including advanced age, disability at presentation, cord diameter, cord area, altered cord signal on $\operatorname{MRI}^{12}\left(\mathrm{~T}_{2^{-}}\right.$and $\mathrm{T}_{1}$-weighted images), increased cervical spinal mobility ${ }^{9}$ and the presence of a congenitally narrow spinal canal. ${ }^{13,14}$ The same factors may determine the response to surgery either positively (increased cervical mobility) or negatively (advanced age, congenitally narrow spinal canal).

We have recently undertaken a systematic review of the evidence from randomized controlled trials of the role of surgery in the treatment of cervical spondylotic radiculopathy and myelopathy. ${ }^{15} \mathrm{We}$ found no studies comparing conservative with surgical treatment in patients with cervical myelopathy. There were two reports by the same authors ${ }^{16,17}$ on a single cohort of patients with cervical radiculopathy where surgery was compared with physiotherapy and cervical collar. Surgical treatment was associated with a better control of pain at 3 months, but conservatively managed patients improved to a similar level at one year. When we compared randomised controlled trials comparing discectomy with fusion there was no difference in various outcomes in two of three studies., ${ }^{5,18}$ In one study, recruiting only patients with soft disc prolapse, more patients returned to work at 9 weeks after discectomy than fusion ${ }^{19}$ This highlights the problems of using rather arbitrary outcome measures in small numbers of patients.

Marks' advice to select the appropriate procedure on the basis of the "available knowledge" is surely not enough for the evidence hungry purchasers of the next millenium. ${ }^{20} \mathrm{~A}$ suitably large prospective randomized study, comparing early vs delayed surgery would define which patients benefit from operation. Neurosurgeons need to provide unbiased and valid evidence with which to answer this important issue, before it is decided for them.

I. P. Fouyas \& P. F. X. STATHAM Department of Clinical Neurosciences, University of Edinburgh, Western General Hospital, Edinburgh EH4 $2 X U$, UK 


\section{References}

1 Marks SM. Cervical degenerative disease: discectomy or fusion? Br $\mathcal{F}$ Neurosurg 1998;12:109-12.

2 Thomas AMC. The spine. In: Pynsent P, Fairbank J, Carr A. eds, Outcome measures in Orthopaedics. Oxford: Butterworth Heinmann, 1993.

3 Cloward R. The anterior approach for the removal of ruptured cervical discs. $\mathcal{F}$ Neurosurg 1958;15:602-17.

4 Bishop RC, Moore KA, Hadley MN. Anterior cervical interbody fusion using autogeneic and allogeneic bone graft substrate: a prospective comparative analysis. $\mathcal{f}$ Neurosurg 1996;85:206-10.

5 van den Bent MJ, Oosting J, Wouda EJ, van Acker $\mathrm{EH}$, Ansink BJ, Braakman R. Anterior cervical discectomy with or without fusion with acrylate. A randomized trial. Spine 1996;21:834-9.

6 Lees F, Aldren Turner JW. Natural history and prognosis of cervical spondylosis. BMf 1963;2:1607-10.

7 Clarke E, Robinson PK. Cervical myelopathy: a complication of cervical spondylosis. Brain 1956;79:483510.

8 LaRocca H. Cervical spondylotic myelopathy: Natural history. Spine 1988;13:854-5.

9 Barnes MP, Saunders $M$. The effect of cervical mobility on the natural history of cervical spondylotic myelopathy. $\mathcal{f}$ Neurol Neurosurg Psychiatry 1984;47:17-20.

10 Nurick S. Natural history and results of surgical treatment of the spinal cord disorder associated with cervical spondylosis. Brain 1972;95:101-8.

11 Radhakrishnan K, Litchy WJ, O' Fallon M, Kurland LT. Epidemiology of cervical radiculopathy. A population-based study from Rochester, Minnesota, 1976 through 1990. Brain 1994;117:325-35.

12 Mehalic TF, Pezzuti RT, Applebaum BI. Magnetic resonance imaging and cervical spondylotic myelopathy. Editorial comment. Neurosurgery 1990;26:21727.

13 Clifton AG, Stevens JM, Whitear P, Kendall BE. Identifiable causes for poor outcome in surgery for cervical spondylosis. Post-operative computed myelography and $\mathrm{MR}$ imaging. Neuroradiology 1990;32:450-5.

14 McCormack BM, Weinstein PR. Cervical spondylosis-an update. West $\mathcal{F}$ Med 1996;165:43-51.

15 Fouyas I, Sandercock P, Statham P, Lynch C. Systematic review of surgery for cervical spondylotic myelopathy and radiculopathy. In: Cochrane Library. Update Software. Protocol.

16 Persson LCG, Carlsson C-A, Carlsson JY. Long-lasting cervical radicular pain managed with surgery, physiotherapy, or a cervical collar. Spine 1997;22: 751-8.

17 Persson LC, Moritz U, Brandt L, Carlsson CA. Cervical radiculopathy: pain, muscle weakness and sensory loss in patients with cervical radiculopathy treated with surgery, physiotherapy or cervical collar. A prospective, controlled study. Eur Spine f 1997;6:25666.

18 Martins AN. Anterior cervical discectomy with and without interbody bone graft. $\mathcal{f}$ Neurosurg 1976;44:290-5.

19 Rosenørn J, Hansen EB, Rosenørn M-A. Anterior cervical discectomy with and without fusion. A prospective study. $\mathcal{F}$ Neurosurg 1983;59:252-5.

20 US Department of Health and Human Services. Agency for Health Care Policy and Research. Acute Pain Management: Operative or Medical Procedures and Trauma. Clinical Practice Guideline No. 1. AHCPR Publication No. 92-0023. Rockville (MD): The Agency; 1993:107.

\section{Reply from Mr Marks}

SIR-Mr Statham makes the comment that I missed two fundamental issues. The first was to define which patients benefited from surgery, and secondly how we might measure the outcome in an unbiased and validated way. Both these are indeed very important and fundamental issues facing the question of surgery in cervical degenerative disease; however, the editorial considered the aspects of the point made in the title, that is discectomy or fusion. The editorial did not set out to discuss the extremely important point of which patients would benefit from surgery and of course at which stage surgery might be the most advantageous. This point in itself would be sufficient for an editorial.

I entirely agree with $\mathrm{Mr}$ Statham regarding the comments on measurement of outcome and that the condition is a heterogeneous one. For this reason studies must specifically consider the sub groups with all the specific aspects of which $\mathrm{Mr}$ Statham mentions a few.

The final paragraph of the editorial suggested that although there are many surgeons who at present continue to perform only one procedure for all conditions, a good case could be made for selecting the appropriate procedure for the patient in question on the basis of available knowledge. The message clearly urges surgeons at present to extend the repertoire and tailor the operation to the patient on the basis of our present understanding and by no means suggests that the available repertoire will be sufficient for the "hungry purchasers of the next millenium"; as Mr Statham suggests.

\section{S. M. MARKS \\ Middlesbrough General Hospital, Middlesbrough, Cleveland TS5 5AZ, UK}

\section{"The pallidotomy debate": are micro-elec- trode-guided pallidotomies always located in the pallidum?}

SIR-We congratulate C. B. Carroll et al. for their paper "The pallidotomy debate" [Carrol CB, Scott $\mathrm{R}$, Davies LE, Aziz T. The pallidotomy debate, $\mathrm{Br}$ f Neurosurg 1998;12(2):146-50]. This paper is an important contribution clarifying some of the controversies about the role of micro-electrode techniques in pallidotomy. We would like to join that debate by focusing on one important aspect on which the authors did not elaborate, concerning the accuracy of micro-electrode-guided lesion placement: on page 149 the authors wrote: "For final lesion location to be determined objectively, postmortem examination with retrospective correlation with clinical outcome needs to be carried out"

We believe that final lesion location can indeed be "determined objectively" by carefully studying pub- 
lished MRI pictures of pallidotomy lesions. ${ }^{1}$ One does not need to wait that operated patients die, God forbids! We therefore urge the authors to study carefully the published figures depicting MRIlesions illustrating papers on microelectrode pallidotomy:

Dogali et al. published in Neurology in April 1995 one MRI figure (Fig. 2, page 755) illustrating their paper "Ventral posterior pallidotomy". ${ }^{2}$ This figure showed a sagittal and coronal picture of a microelectrode-guided pallidotomy lesion claimed to lie within $1 \mathrm{~mm}$ of the target. While the sagittal picture is of no use as far as assessment of lesion location is concerned, the coronal picture depicted a lesion that was neither posterior nor ventral. In fact, the lesion was very dorsal and very anterior since the optic chiasm, which lies about $10 \mathrm{~mm}$ anterior to the target was indeed visible on that coronal picture. The same team published another micro-electrodeguided MRI-pallidotomy figure illustrating each of two other papers that appeared in Advances in Neurology (Fig. 1, p. 586) in $1996^{3}$ and in Stereotactic and Functional Neurosurgery (Fig. 2, p. 166) in April 1997. ${ }^{4}$ This figure showed a ventral and posterior, but extremely lateral pallidal lesion, which was definitely located in the Gpe, and not as intended in the GPi, as can be assessed from the distance of that lesion to the visible optic tract on the figure. Lozano et al. published in Acta Neurochirurgica, Suppl 64, (Fig. 1B, p. 31) in 1995, a figure showing a microelectrode-guided posteroventral pallidotomy lying very anterior in the pallidum. ${ }^{5}$

Another paper of Lozano et al. appeared in the November 1997 issue of Movement Disorders. ${ }^{6}$ The MRI figure in that paper, depicting micro-electrodeguided pallidotomies done bilaterally in a dystonia patient (Fig. 2, p. 868), shows very clearly that the right-sided "pallidotomy" lesion is in the internal capsule, just medial from the visible medial border of the pallidum. The contralateral lesion is clearly more lateral, lying at the intended Gpi target.

Another micro-electrode-guided pallidotomy lesion illustrated a paper by Samuel et al. published in Brain in 1988: ${ }^{7}$ Figure 1 B, on p. 64 of that paper shows a posteroventral pallidotomy lesion located so medial in the GPi that roughly half the lesion in fact lies in the internal capsule.

The Emory group at Atlanta had never published MRI pictures depicting their lesions. Instead, artist drawings of pallidal lesions were used to illustrate several of their articles about micro-electrodeguided pallidotomy. ${ }^{8-10}$ Only recently, in January 1998, a paper from the same group published in Neurology, ${ }^{11}$ discussing visual fields following microelectrode pallidotomy, was illustrated with two figures showing MRI pallidotomies and their relation to the optic tract.

Furthermore, in two recent publications on micro-electrode-guided pallidotomies, an unacceptable rate of mortality and severe morbidity was re- ported, ${ }^{6,12}$ and the authors of one of these papers ${ }^{12}$ actually warned against pallidotomy: the paper by Shannon et al. ${ }^{12}$ published in February 1998(!), was concluded by the following sentence: "We are concerned that the procedure will gain widespread use outside of PD research centers before its efficacy and safety have been better defined".

We conclude that published good quality MRI illustrations do indeed allow for objective assessment of location of pallidal lesions. The claim that micro-electrode technique is the method of choice to ensure a proper placement of the lesion cannot be sustained when analysing the great disparity in location of published micro-electrode-guided pallidotomy lesions as assessed on MRI, some of which appear not to lie in the pallidum.

Notwithstanding the risks of multiple tracks in the brain and the risks of a prolonged surgery in terms of infection risk and fatigue of the patient, affecting negatively his/her cooperation during surgery, micro-electrode techniques may not constitute a guarantee for proper placement of the pallidal lesions, nor for minimizing side-effects, as shown in several recent publications. Therefore, in our opinion, if micro-electrode-guided pallidotomy does not constitute a better means than macro-stimulation to obtain a correctly located pallidotomy lesion, and if it harbours increased risks for serious side-effects, then it should be used only in expert hands and for scientific purpose, and not for routine procedures in the widespread practice of pallidotomy. Besides, patients undergoing micro-electrode-guided pallidotomy should be informed of, and consent to, the additional risks involved with that technique, compared with macro-stimulation techniques.

\section{I. HARIZ \\ Department of Neurosurgery, University Hospital, Umeå, Sweden \\ H. FODSTAD Division of Neurosurgery, New York, USA} New York Methodist Hospital-Cornell Medical Center,

\section{References}

1 Hariz MI. Controversies in pallidal surgery. Acta Neurochir (Wien) Suppl 1997;68:1-10.

2 Dogali M, Fazzini E, Kolodny E, Eidelberg D, Sterio $\mathrm{D}$, Devinsky O, et al. Stereotactic ventral pallidotomy for Parkinson's disease. Neurology 1995;45:753-61.

3 Dogali M, Sterio D, Fazzini E, Kolodny E, Eidelberg $D$, Beric A. Effects of posteroventral pallidotomy on Parkinson's disease. In: Battistin L, Scarlato G, Caraceni T, Ruggieri S, eds, Advances in Neurology, Vol. 69. Philadelphia: Lipincott-Raven, 1996:585-90.

4 Beric A, Sterio D, Dogali M, Alterman R, Kelly P. Electrical stimulation of the globus pallidus preceding stereotactic posteroventral pallidotomy. Stereotact Funct Neurosurg 1996;66:161-9. 
5 Lozano AM, Hutchinson WD, Dostrovsky JO. Microelectrode monitoring of cortical and subcortical structures during stereotactic surgery. Acta Neurochir (Wien) Suppl 1995;64:30-4.

6 Lozano AM, Kumar R, Gross RE, Giladi N. Hutchison WD, Dostrovsky JO. et al. Globus pallidus internus pallidotomy for generalized dystonia. Mov Disord 1997;12:865-70.

7 Samuel M, Caputo E, Brooks DJ, Schrag A, Scaravilli T, Branston NM, et al. A study of medial pallidotomy for Parkinson's disease: clinical outcome, MRI location and complications. Brain 1998;121:59-75.

8 Baron MS, Vitek JL, Bakay RAE, Green J, Kaneoke Y, Hashimoto T. et al. Treatment of advanced Parkinson's disease by posterior GPi pallidotomy: 1-year results of a pilot study. Ann Neurol 1996;40:355-66.

9 Vitek JL. Stereotaxic surgery and deep brain stimulation for Parkinson's disease and movement disorders. In: Watts RL, Koller WC. eds, Movement Disorders. New York: McGraw Hill, 1997:237-55.

10 Vitek JL, Bakey RAE, DeLong MR. Microelectrodeguided pallidotomy for medically intractable Parkinson's disease. In: Obeso JA, DeLong MR, Ohye C, Marsden CD. eds, The Basal Ganglia and New Surgical Approaches for Parkinson's Disease, Advances in Neurology, Vol 74. Philadelphia: Lipincott Raven, 1997:183-98.

11 Biousse V, Newman NJ, Carroll C, Mewes K, Vitek $\mathrm{JL}$, Bakay RAE. et al. Visual fields in patients with posterior Gpi pallidotomy. Neurology 1998;50:258-65.

12 Shannon KM, Penn RD, Kroin JS, Adler CH, Janko KA, York M. et al. Stereotactic pallidotomy for the treatment of Parkinson's disease. Efficacy and adverse effects at 6 months in 26 patients. Neurology 1998;50:434-8.

\section{Reply to Hariz \& Fodstad}

SIR-We would like to thank Drs Hariz and Fodstad for their helpful comments in response to our recent publication "The Pallidotomy Debate" [Carroll CB, Scott R, Davies LE, Aziz TZ. The Pallidotomy Debate. $B r f$ Neurosurg 1998;12(2):146-50] and would wish to add a few comments of our own in response to the issues raised by them.

We would agree with all points raised in their letter, but still feel that with long-term follow-up of patients, opportunities shall arise, albeit intermittently, to confirm lesion placement histologically, and that on all such occasions centres should avail themselves of these chances as histology must remain the gold standard for lesion site conformation. We recognize that these numbers will be small, however, and agree that postoperative MRI scans are the next best option.
Hariz states that micro-electrode recording (MER) does not appear to increase the accuracy of lesions as far as can be assessed from published figures, and we would certainly be in concurrence on this point. However, more interesting is that in the studies published to date concerning outcome vs lesion site and size, no obvious correlation has been demonstrated. ${ }^{1,2}$ We would suggest from currently published data and our own experience that all that may be said at present is, that for prolonged effect lesion volume should be $60-100 \mathrm{~mm}^{3}$ (larger lesions may have a better motor effect at the expense of cognitive deficits), the lesion should be located mostly in the medial pallidum, GPi (a great deal of encroachment into the lateral pallidum, GPe, may exacerbate symptoms ${ }^{3}$ ) and lesions should be in the posterior half of GPi. If no further degree of specificity is needed than that outlined, then MER may, indeed, be considered as unnecessary. Of course, the optic tract and internal capsule must be avoided; however, this is safely achieved by the use of impedance monitoring and macro-stimulation.

It may well be that further publications regarding lesion analysis will take us forward from this point, and closer to resolving the debate regarding the best means of target identification. At present, however, no emphatic statements can be made regarding the optimum technique for target acquisition, and the difficulty of reliable post operative lesion analysis remains unsolved.

\section{T. Z. Aziz \& L. E. MunRo-Davies Department of Neurosurgery Radcliffe Infirmary, Oxford OX2 6HE, UK}

\section{References}

1 Hariz MI. Correlation between clinical outcome and size and site of lesion in computed tomography guided thalamotomy and pallidotomy. Stereotact Funct Neurosurg (Proceedings of the $\mathrm{Xth}$ meeting of the world society for stereotactic and functional neurosurgery, Maebashi, Japan, October 1989) 1990;54:172-85.

2 Burns JM, Wilkinson S, Kieltyka J, Overman J, Lundsgaarde $\mathrm{T}$, Tollefson $\mathrm{T}$. et al. Analysis of pallidotomy lesion positions using three-dimensional reconstruction of pallidal lesions, the basal ganglia, and the optic tract. Neurosurgery 1997;41(6):1303-19.

3 Munro-Davies LE, Gregory R, Squires W, Radatz M, Silburn P, Scott R. et al. Lateral pallidotomy exacerbates akinesia in the Parkinsonian patient: a case report. f Clin Neurosci 1999;6(2). 


\title{
Use of the Radionics Image Fusion ${ }^{\mathrm{TM}}$ and Stereoplan ${ }^{\mathrm{TM}}$ programs for target localization in functional neurosurgery
}

\author{
Vakis Papanastassiou ${ }^{1}$ MD fRCS(SN), Jeremy Rowe ${ }^{1}$ FRCS, R. Scott' PhD, P. Silburn ${ }^{2}$ FRACP PhD, \\ L. Davies' FRCS, Tipu Aziz' ${ }^{1}$ MD FRCS(SN) \\ ${ }^{1}$ Department of Neurosurgery, Radcliffe Infirmary, Woodstock Road, Oxford OX2 6HE, UK \\ ${ }^{2}$ Department of Neurology, Princess Alexandra Hospital, Brisbane, Australia
}

We describe the use of Radionics Image Fusion ${ }^{\mathrm{TM}}$ and Stereoplan ${ }^{\mathrm{TM}}$ in defining the target for thalamotomy and pallidotomy in functional surgery for parkinsonism and tremor. Using this to fuse and spatially correct magnetic resonance imaging (MRI) to computed tomography $(\mathrm{CT})$ images our calculated targets were a mean of $0.6 \pm 1.5 \mathrm{~mm}$ from the end target determined physiologically by stimulation. This is significantly better than the values of $2.6 \pm 1.6 \mathrm{~mm}$ for thalamic targets and $7.1 \pm 3.7 \mathrm{~mm}$ for pallidal targets using CT alone. As a consequence, determination of the target and the lesion making are routinely performed in one pass of the electrode allowing for faster, more accurate and, we believe, safer functional procedures.

Journal of Clinical Neuroscience 1998, 5(1): 28-32

(c) Harcourt Brace \& Co. Ltd 1998

Keywords: Parkinson's disease; tremor; image fusion; stereoplan; functional neurosurgery

\section{Introduction}

Recently there has been a phenomenal increase in the practice of functional neurosurgery particularly for the tremor, rigidity and drug-induced dyskinesias of Parkinson's disease (PD) and for tremor of other aetiologies. Critical to the success of this surgery and to the avoidance of complications is the precise placement of lesions. Ideally, targets should be tailored to the patient's pathology, symptomatology and to any individual variations in anatomy. The best way to achieve this is the subject of some debate, with considerable discussion about the relative spatial accuracy of computed tomography (CT) versus magnetic resonance imaging (MRI) scanning.

The use of CT scans in stereotaxy is well established and accuracy within $\pm 1.0 \mathrm{~mm}$ in the $\mathrm{x}$ and $\mathrm{y}$ and $\pm 1.5 \mathrm{~mm}$ in the $\mathrm{z}$ coordinates can be obtained; this reflects the linear nature of CT scan acquisitions. Tissue contrast, however, is limited, with no delineation of the deep grey matter nuclei from the white matter tracts. Conversely, MRI offers superb anatomical definition, but the use of non-linear magnetic field gradients and the existence of field inhomogeneities may introduce spatial errors. Comparisons of CT and MRI suggest that discrepancies of up to $10 \mathrm{~mm}$ may occur; ${ }^{1-4}$ errors of that order are critical in situations such as radiosurgery and functional neurosurgery where the safety margin in target calculations is quite often under $5 \mathrm{~mm}$.

In an effort to circumvent the problems arising from the inherent geometric inaccuracies of MRI scanning, whilst at the same time preserving the superior tissue definition that it offers, we used the algorithm described by Eben Alexander III et $\mathrm{al}^{5}$ for fusing preoperative MRI scans with CT scans obtained with the patient in the Cosman-Roberts-Wells (CRW) stereotactic frame. This generates a fused image where the final image is that of the stereotactic CT scan bone window with the MRI soft tissue detail. The anterior (AC) and posterior ( $\mathrm{PC}$ ) commissures and the medial pallidum can be readily identified.

The computer program also incorporates the Schaltenbrand functional atlas. ${ }^{6}$ This is scaled to the AC-PC distance in the fused image, and a lateral scaling factor is also used to correct for variations between individual brains. We report our experience at Oxford using this technology and compare it to the traditional method for calculating functional target coordinates.

\section{Materials and methods}

Between July and November 1995 we used the following technique in 23 stereotactic functional procedures. A summary of the patient details, symptoms, targets and results is given in the Table. We use a CRW stereotactic frame, a Hewlett Packard HP7000 workstation with the Image Fusion ${ }^{\mathrm{TM}}$ and Stereoplan ${ }^{\mathrm{TM}}$ software applications and a Radionics FG-3C radio-frequency lesion generator (Radionics, MS, USA).

Prior to surgery, patients had three self-adhesive, CT/MRI detectable markers affixed to the glabella and both parietal eminences. An axial T1-weighted MRI scan is then performed, using a three-dimensional (3D) turbo-FLASH sequence (TE $7 \mathrm{~ms}$, TR $15 \mathrm{~ms}$ ) on a Siemens 1.5 Tesla Magnetom Vision system, 3-mm thick contiguous slices being acquired. At surgery the CRW 
Table Patient clinical details and chosen targets

$\begin{array}{lclll}\text { Diagnosis } & \text { No. } & \text { Main symptoms } & \text { Target } \\ \text { PD } & 5 & \text { Resting tremor } & \text { VIM thalamus } \\ \text { PD with L-Dopa dyskinesia } & 16 & \text { Dyskinesia, on-off, freezing } & \text { Posterior half medial pallidum Alleviation (bilateral in 6/16) } \\ \text { Benign, essential tremor } & 1 & \text { Resting tremor } & \text { VIM thalamus } \\ \text { Multiple sclerosis } & 2 & \text { Intention tremor } & \text { VOP thalamus } \\ \text { Post-stroke/trauma intention tremor } & 1 & \text { intention tremor } & \text { VOP thalamus }\end{array}$
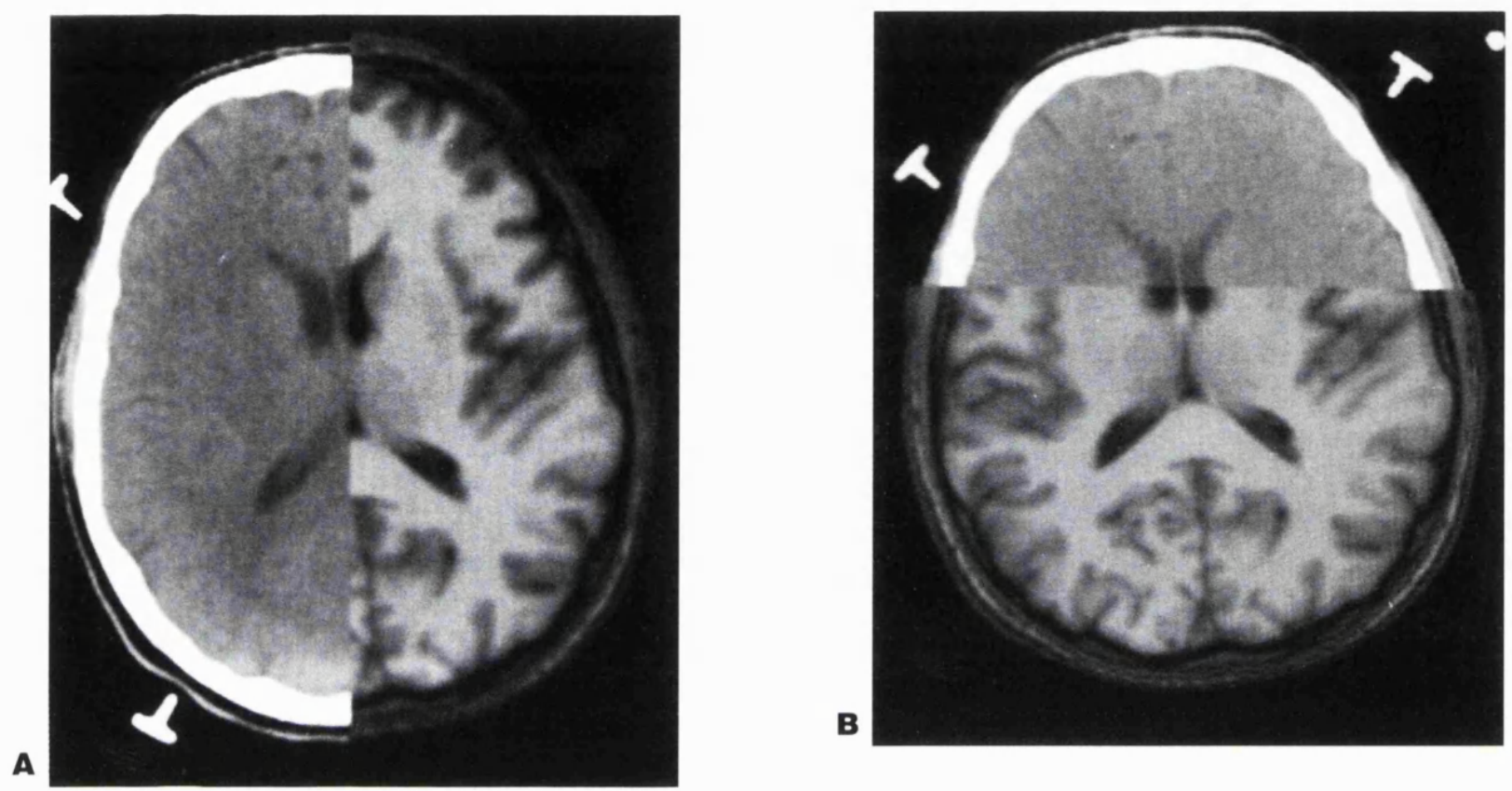

Fig. 1 (A, B) Split images showing the accuracy of Image Fusion by overlaying CT and MR data. The subarachnoid space and various anatomical structures are shown in continuity between the two halves.

frame is fixed to the patient's skull under sedation and local anaesthesia. Two-millimetre thick contiguous CT scans are then acquired covering the thalamus, basal ganglia and the three markers, the scans oriented to include the $\mathrm{AC}$ and the $\mathrm{PC}$ in the same slice.

The MR and CT data files are transferred to the workstation in the operating theatre and fused using the Image Fusion ${ }^{\mathrm{TM}}$ facility within the Stereoplan ${ }^{\mathrm{TM}}$ program. Initially the external markers are co-registered to align the images. Following this, segmentation of the voxels that lie on bony surfaces in both CT and MRI identification of these $3 \mathrm{D}$ coordinates is performed, using spectral analysis of CT and MR bony windows and thus creating a template for the subsequent matching. The MR images are then spatially corrected to CT template. The accuracy of fusion can be checked in two ways: a split window allows overlaving of the CT and the adjusted MRI slices so that a visual check can be obtained (Fig. 1A, B); in addition, multiple anatomical structures are selected independently on the CT images and on the MRI data fused to the CT scans, the correspondence being checked. The whole process takes approximately $25 \mathrm{~min}$ and is performed in theatre whilst the patient is being prepared.
Target coordinates are calculated using two different methods:

\section{From the CT data alone.}

The coordinates of the $\mathrm{AC}$ and $\mathrm{PC}$ are determined, and hence those of the mid-commissural point, from which targets have been traditionally obtained in functional neurosurgery: VIM at the same horizontal plane, 13-15 $\mathrm{mm}$ lateral and $3-5 \mathrm{~mm}$ posterior to the midcommissural point; VOP at the same horizontal and coronal planes and $13-15 \mathrm{~mm}$ lateral; the medial pallidum at $3-7 \mathrm{~mm}$ inferior, $20-21 \mathrm{~mm}$ lateral and $2 \mathrm{~mm}$ anterior to the same.

\section{Using the fused image and the Stereoplan ${ }^{\mathrm{TM}}$} application.

The AC and PC, which can be more easily and accurately identified on the fused image (Fig. 2), are used to adjust the orientation of the Shaltenbrand stereotactic atlas and provide a geometric scaling factor in the antero-posterior direction. A lateral correction factor is also obtained, by co-registering an easily identifiable anatomical structure and its atlas equivalent, the apex of the medial pallidum, the ventricular surface or the border 


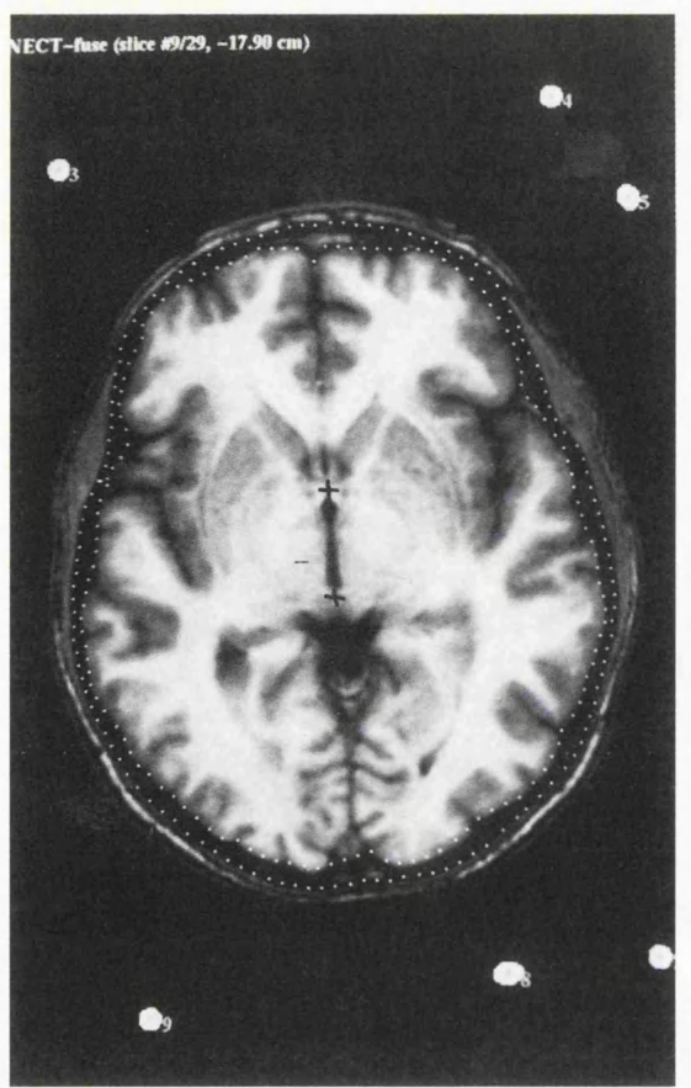

Fig. 2 Fused axial scan demonstrating the anterior commissure (AC) and posterior commissure (PC), providing a much improved visual identification. between the putamen and the lateral pallidum being generally used (Fig. 3). The accuracy with which the atlas is fitted to the fused CT-MR data is verified by selecting various anatomical landmarks on the fused image window and comparing them to the position of the marker on the atlas window. Adjustments to the lateral correction factor can be made at this point if necessary. The target is then selected visually on the fused image and the atlas is used to fine tune the coordinates and determine the anatomical extent of the target boundaries and an optimal approach (Fig. 4). Comparing the preoperative plan (Fig. 4) with the postoperative plan (Fig. 5) for a pallidotomy confirms accurate placement of the lesion.

A $3.0 \mathrm{~mm}$ twist-drill is placed along the coronal suture, $2 \mathrm{~cm}$ lateral to the midline for thalamotomy and $6 \mathrm{~cm}$ for medial pallidotomy, and the target coordinates are set on the CRW frame. The final physiological determination of the target is done by means of stimulation with the patient awake. We use a $1.8-\mathrm{mm}$ diameter, $2.0 \mathrm{~mm}$ exposed tip electrode and begin to study responses from $5.0 \mathrm{~mm}$ proximal to $5.0 \mathrm{~mm}$ distal to the calculated target along the electrode trajectory. Target confirmation for thalamotomy is that of arrest or aggravation of tremor at $60-100 \mathrm{~Hz}$ with $1.5 \mathrm{~V}$. For medial pallidotomy, localization combines impedance monitoring with stimulation. With the electrode we use, the imperlance of the medial pallidum is $500-700 \mathrm{ohms}$ with a sharp rise to $1000-1100$ ohms in the ansa lenticularis. A-P localization to the posterior medial pallidum is obtained by stimulating at $2 \mathrm{~Hz}$ until co-contraction of the contralateral mouth and hand are obtained at $2.0 \mathrm{~V}$. Impedance at this point is about $500-700$ ohms. Gradually advancing the electrode until the impedance rises indicates that the ansa lenticularis is reached at which point stimulation at $2.0 \mathrm{~V}$ leads to contralateral

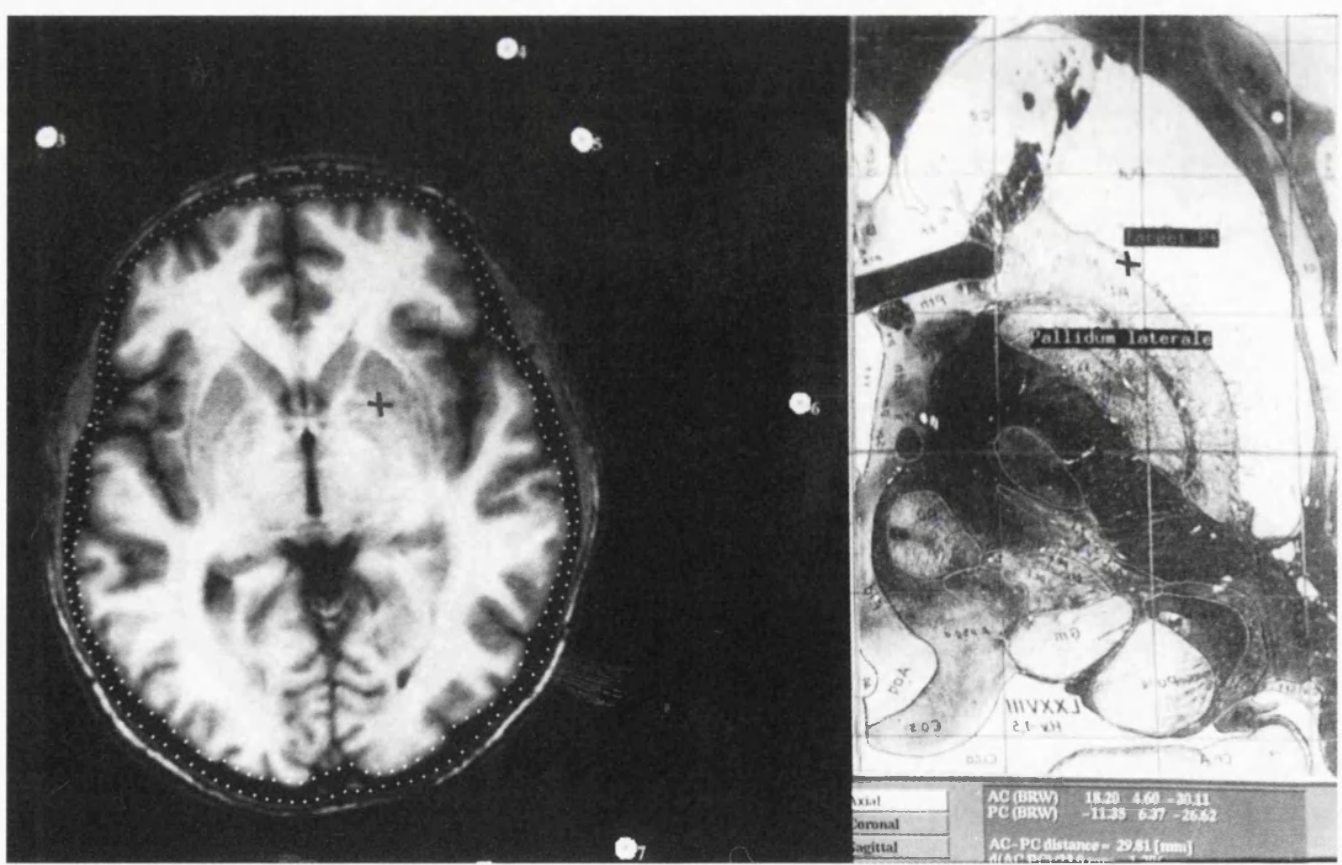

Fig. 3 View of the workstation screen. Lateral correction is performed by matching easily identified anatomical boundaries (in this case the putamino-pallidal junction) on the fused MRI/CT scan and the functional atlas. 


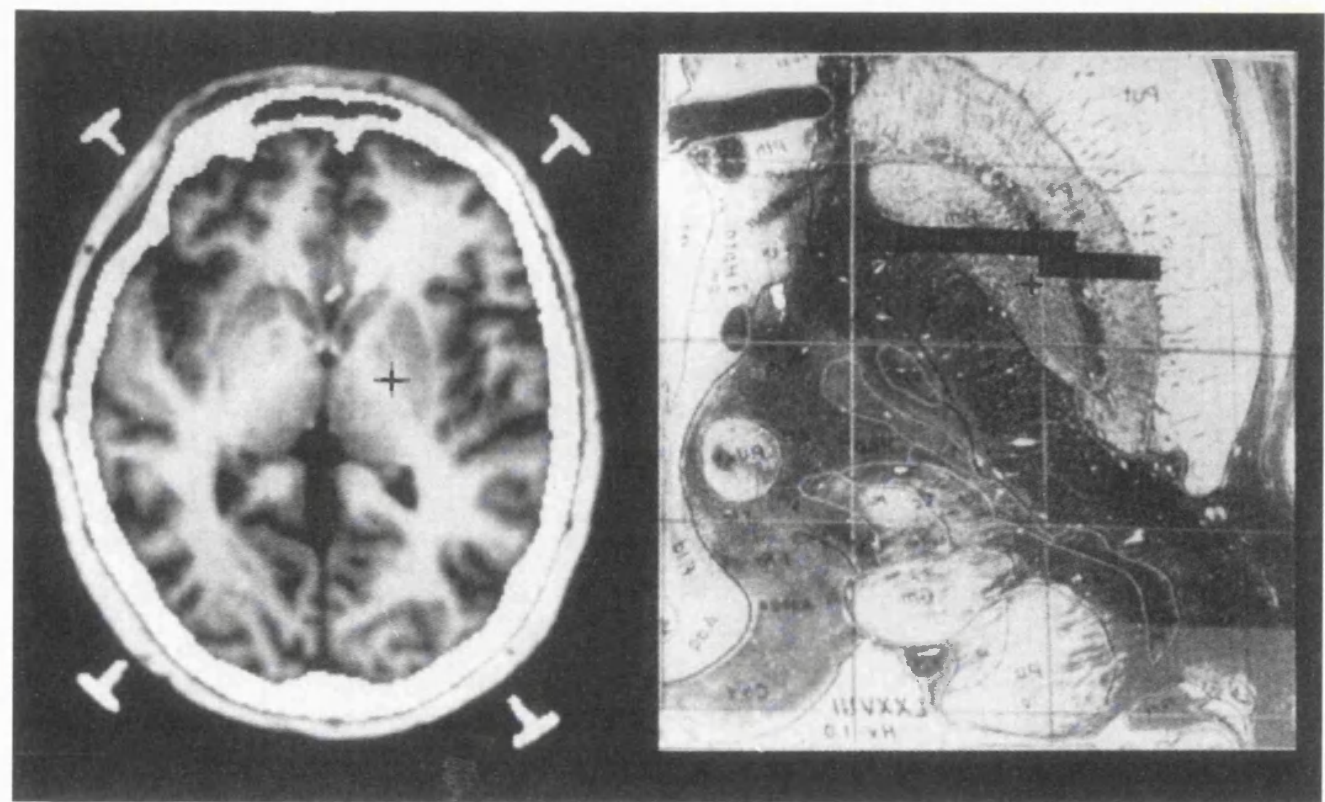

Fig. 4 The chosen target is selected off the fused MRI/CT scan with confirmatory co-localization on the functional map.

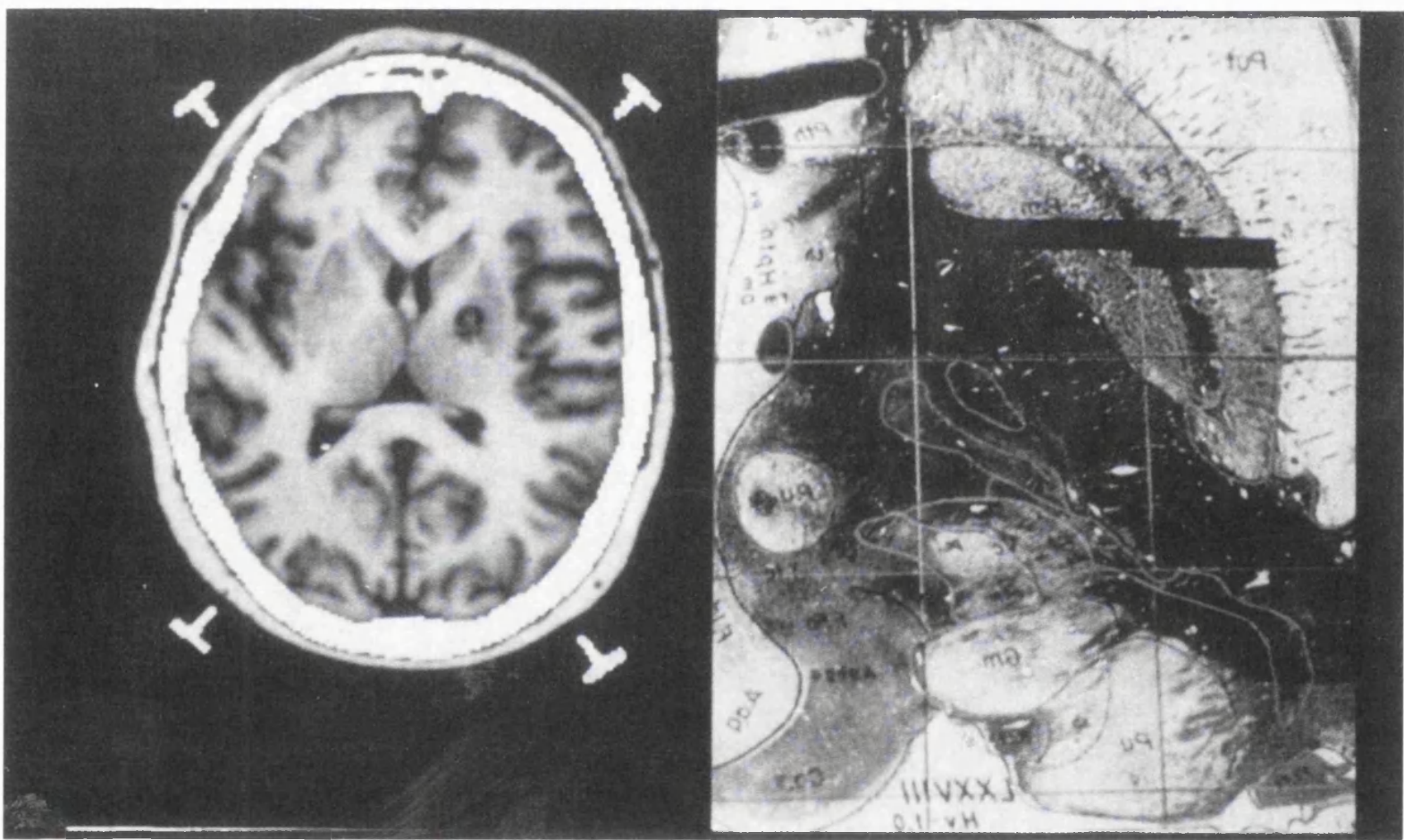

Fig. 5 Fused postoperative MR scan of a patient in our series having undergone a stereotactic medial pallidotomy, showing the lesions in the correct position and confirmed in the atlas.

visual phosphenes; we do not proceed any more ventral to this point. The first lesion is made at $60^{\circ} \mathrm{C}$ for $30 \mathrm{~s}$ and the electrode retracted in $2 \mathrm{~mm}$ steps; the second lesion is made at $75^{\circ} \mathrm{C}$ for $60 \mathrm{~s}$, the third at the same and the fourth at $75^{\circ} \mathrm{C}$ for $90 \mathrm{~s}$. If the arm and leg have both improved then the lesion is of a satisfactory size and in some cases may not require all the described lesions to be made.

During pallidotomy the effects of stimulation are variable; we look for alleviation or exacerbation of rigiclity, increased fluidity of movement and loss of bradvkinesia with stimulation at $50-100 \mathrm{~Hz}$.

\section{Results}

For the purposes of this study, we looked at two separate issues: a direct comparison between C.T alone and fused CT-MR in determining the $\mathrm{AC}$, the $\mathrm{PC}$ and the AC-PC. length; and the discrepancy between the final target, as determined physiologically by stimulation (when multiple lesions were made, the geometric average of these targets) and the target coordinates as derived by the two methods.

We found that when using the fused CT-MR image, the AC was localized significantly more anterior 
$(1.1 \pm 1.4 \mathrm{~mm}, P<0.01)$, and significantly more inferior $(2.6 \pm 2.3 \mathrm{~mm}, P<0.01)$, than when the CT data alone was used. There was an overall discrepancy of $3.5 \pm 1.7 \mathrm{~mm}$ between the AC coordinates as determined by the two methods. The overall discrepancy in determining the PC between the two methods was $2.8 \pm 2.2 \mathrm{~mm}$, similar to that for the AC although the error did not occur consistently in any particular direction. There was no statistically significant difference in the AC-PC length as calculated by the two methods.

Comparing the target coordinates, we found the fused data significantly more accurate than CT alone $(P<0.01)$ in determining targets for ablation, with an overall discrepancy of $0.6 \pm 1.5 \mathrm{~mm}$ between the Stereoplan-fusion calculated and the physiological target, compared to a difference of $5.6 \pm 3.8 \mathrm{~mm}$ between the traditional CT calculations and the lesion position. The CT alone calculations are significantly more accurate for thalamic than pallidal targets $(2.6 \pm 1.6 \mathrm{~mm}$ compared to $7.1 \pm 3.7 \mathrm{~mm}, P<0.01$ ). There was no difference between thalamic and pallidal target accuracy when using the CT-MR fused data, in both cases the mean discrepancy being less than $0.6 \mathrm{~mm}$.

\section{Discussion}

Physiological localization has been achieved in two ways: stimulation and recording. The latter has been refined to single unit recordings using micro-electrodes. With the recent increase in selective medial pallidotomy for parkinsonism, the issue raised has been that of the necessity of unit recording for localization. The pioneering work of groups such as Iacono, Lozano, Dogali and DeLong ${ }^{7-10}$ have established the physiological target, which corresponds with a well-defined anatomical structure. This can be identified accurately with image fusion and stimulation, thus providing an alternative and we believe, equally valid, localization technique.

Certainly, the lesions made at our unit correspond very closely with those made using micro-electrode recording, both in size and position (Fig. 5). We have also found that because of the more precise anatomical calculation of target coordinates, there has been a dramatic reduction in the number of stimulation positions before the physiological target is identified, as shown by the close proximity of the lesion to the target calculated using Image Fusion ${ }^{\mathrm{TM}}$. This has made the operation both faster and safer, as fewer electrode passes through brain substance are required. The average time for the whole procedure, from placing the CRW frame on to the patient's head to completion, has been $3 \mathrm{~h}$.

With the resurgence in functional neurosurgery for movements disorders, a need has arisen for a reliable, accurate, quick and easy to use method of target acquisition. The increasing experience with functional neurosurgery and the better understanding of basal ganglia physiology suggest that clearly defined anatomical targets, such as the posterior half of the medial pallidum or the sub-thalamic nucleus, can provide significant clinical results. The current generation of stereotactic frames provides accuracy in the order of $1.0 \mathrm{~mm}$ and the localization method needs to be approaching that order of magnitude. The use of image fusion has allowed us to visually acquire targets from the MR data, whilst maintaining the proven geometric fidelity of CT. The availability of the Schaltenbrand atlas in the Stereoplan software application adds an extra dimension to both the planning capability for target selection and the accuracy of the calculation. We, in our experience, find that accurate anatomical localization with careful stimulation and intraoperative assessment, results in a quick, safe and effective procedure.

Recieved 13 August 1997; Accepted 8 September 1997

Correspondence and offprint requests: $\mathrm{Mr} \mathrm{T}$. Z. Aziz, Department of Neurosurgery, Radcliffe Infirmary, Woodstock Road, Oxford OX2 6HE, UK, Tel: 441865 228425, Fax: 441865224898

\section{References}

1. Kondziolka D, Dempsey P, Lunsford L, Kestle J, Dolan E, Kanal E, Tasker R. A comparison between magnetic resonance imaging and computed tomography for stereotactic co-ordinate determination. Neurosurgery 1992; 30: 402.

2. Dade Lunsford L, Martinez A, Latchaw R. Stereotaxic surgery with a magnetic resonance and computerised tomography compatible system. J Neurosurg 1986; 64 : 872-878.

3. Gerdes J, Hitchon P, Neerangun W, Torner J. Computed tomography versus magnetic resonance imaging in stereotactic localisation. Stereotact Funct Neurosurg 1994; 62: 124-129.

4. Walton L, Hampshire A, Forster, Kemeny A. A phantom study to assess the accuracy of stereotactic localization, using T1-weighted magnetic resonance imaging with the Leksell stereotactic system. Neurosurgery 1996; 38: 170-176.

5. Alexander III E, Kooy $\mathrm{H}$, van Herk $\mathrm{M}$ et al. Magnetic resonance image-directed stereotactic neurosurgery: use of image fusion with computerised tomography to enhance spatial accuracy. J Neurosurg 1995; 83 : 271-276.

6. Scaltenbrand G, Wahren W. Atlas for Stereotaxy of the Human Brain, 2nd Edn. Stuttgart: Georg Thieme Publishers, 1977.

7. Iacono RP, Shima F, Lonser RR, Kuniyoshi S, Maeda G, Yamada $S$. The results, indications and physiology of posteroventral pallidotomy for patients with Parkinson's disease. Neurosurgery 1995; 36(6): 1118-1125.

8. Lozano A, Hutchison W, Kiss Z, Tasker R, Davis K, Dostrovsky J. Methods for microelectrode guided posteroventral pallidotomy. J Neurosurg 1994; 84(2): 194-202.

9. Sterio D, Beric A, Dogali M, Fazzini E, Alfaro G, Devinsky O. Neurophysiological properties of pallidal neurones in Parkinson's disease. Ann Neurol 35(5): 586-591.

10. Wichmann T, Baron MS, DeLong MR. Local inactivation of the sensorimotor territories of the internal segment of the globus pallidus and the subthalamic nucleus alleviates Parkinsonian motor signs in MPTP-treated monkeys. In: Perceron G, McKenzie JS, Feger J (eds). Ganglia IV. New York: Plenum Press, 1994: 349-355. 


\title{
Surgical complications of functional neurosurgery treating movement disorders: results with anatomical localisation
}

\author{
J. G. Rowe frCS, L. E. Davies frCs, R. Scott PhD, R. Gregory MRCP, T. Z. Aziz MD FRCS(SN) \\ Departments of Neurosurgery and Neurology, Radcliffe Infirmary, Oxford
}

Summary Thalamic and pallidal lesions can alleviate movement disorders, but to achieve this safely and efficaciously requires accurate target localization. We report the surgical complications encountered using an anatomical localization technique to create 121 thalamic and pallidal lesions in 79 consecutive patients over a 3 year period. There was no perioperative mortality, although there was one late death indirectly related to surgery. The risk of haemorrhage was 3.3\% per lesion made. Anatomical localization offers a relatively safe way of identifying targets for functional neurosurgery, with complication rates which compare favourably with the published literature.

Keywords: pallidotomy, thalamotomy, complications

\section{INTRODUCTION}

Highly specific lesions in the thalamus and medial pallidum can effectively alleviate the tremor, rigidity, bradykinesia and drug induced dyskinesia encountered in Parkinson's disease and other movement disorders. ${ }^{1-4}$ In selecting and advising patients about such surgery, neurologists and neurosurgeons need to appreciate the risks and complications associated with making these lesions. Much of the surgical experience and most of the published series predate the levodopa era, when these procedures were commonplace. Since then there have been dramatic changes in tomographic imaging, and potentially this allows targets to be localized more directly and accurately. Even with these advances, contrast ventriculography, defining the anterior and posterior commissures and using a standard atlas to predict the position of nuclei, remains the standard method for anatomical localization, with microelectrode recording being used to confirm the target physiologically. ${ }^{5-7}$ If the target predicted anatomically by this method is some distance from the true and safe one, multiple passes with an electrode may be required to provide the best physiological target . Not only is this process time consuming (operations lasting 4-18 h), but each pass of the electrode incurs the risk of haemorrhage. Reflecting this, one modern series of thalamotomies had a $61 \%$ early and $13 \%$ permanent complication rate, while for pallidotomy, complication rates of up to $100 \%$ have recently been reported. ${ }^{8-12}$ Here we report our experiences with a different localization technique, which combines magentic resonance imaging (MRI) and computed tomography (CT) scanning to define the target anatomically, and uses stimulation in the awake patient to confirm it physiologically. We believe that this approach is not only faster, but because it avoids multiple passes with an electrode, that it is also safer, which is reflected in our complication rates.

Received 20 August 1997 Accepted 2 October 1997

Correspondence to: T. Z. Aziz, Tel: +44 (0) 1865 311188; Fax: +44 (0) 1865 224898

\section{METHODS}

\section{Surgical principles}

Details of the surgical technique have been published elsewhere ${ }^{13}$ but our approach is based on imaging and anatomical localization. MRI has the advantage of allowing grey-white matter tissue contrast, and so delineates the thalamus and the medial pallidum from the internal capsule. Unfortunately there are spatial distortions inherent in MRI which limits the accuracy of directly using it to guide stereotactic procedures. ${ }^{14-16} \mathrm{CT}$ guided stereotaxy is spatially much more accurate but lacks the neuroanatomical definition. To overcome these problems, an MRI scan of the patient is acquired preoperatively, then fused and spatially corrected to the CT scan of the patient acquired during the operation when the stereotactic CRW frame is in place. This process uses the Radionics Image Fusion ${ }^{\mathrm{TM}}$ Stereoplan ${ }^{\mathrm{TM}}$ software. The fused image has the accuracy of the stereotactic space, whilst allowing direct visualization of the thalamus and pallidum. A computerized atlas ${ }^{17}$ can then be fitted to the individual patient's fused image, and the atlas and the fused image are used together to define the target in stereotactic space. ${ }^{18}$ With the patient awake, an electrode is then passed stereotactically down to the target. When stimulation has confirmed the arrest of tremor, or the alleviation of rigidity and akinesia without side-effects, the electrode is used to make a lesion. Generally only a single pass of the electrode is required to identify and lesion a target.

\section{Clinical details}

Between January 1994 and January 1997 we performed 107 operations and attempted to make 121 lesions in 79 patients. Patient details are summarised in Table 1. The VIM nucleus of the thalamus was lesioned 55 times to treat mainly Parkinsonian and benign essential tremor. Fifteen lesions were attempted in the VOP thalamic nucleus for cerebellar tremor secondary to multiple sclerosis. Fiftyone pallidotomies were performed for rigidity, akinesia and druginduced dyskinesia associated with the L-dopa failure syndrome. During this series, 11 bilateral pallidotomies were performed as a single operation, and three combinations of thalamic and pallidal 
Table 1

\begin{tabular}{lcccc}
\hline Condition & $\begin{array}{c}\text { No. of } \\
\text { operations }\end{array}$ & $\begin{array}{c}\text { No. of } \\
\text { patients }\end{array}$ & $\begin{array}{c}\text { Age (years) } \\
\text { Mean (range) }\end{array}$ & $\begin{array}{c}\text { Follow-up (months) } \\
\text { Mean (range) }\end{array}$ \\
\hline Parkinson's disease & 64 & 51 & $59[37-78]$ & $12[2-34]$ \\
Multiple sclerosis & 15 & 12 & $39[28-64]$ & $24[11-38]$ \\
Benign essential tremor & 10 & 9 & $70[66-80]$ & $13[7-20]$ \\
Dystonia & 17 & 6 & $35[17-52]$ & $21[8-33]$ \\
Stroke & 1 & 1 & 69 & 16 \\
Overall & 107 & 79 & $55[17-80]$ & $15[2-38]$ \\
\hline
\end{tabular}

lesions were made. Overall the mean age of these patients was 55 years (range 17-80 years), and the mean follow up 15 months (range 2-38 months). All patients had postoperative scanning to confirm the lesion position. Independent assessments by a neurologist and a neuropsychologist measured quality of life, functional and neurological outcome, and cognitive status.

\section{RESULTS}

In this series there was no perioperative mortality, although there was one death indirectly related to surgery. In this case, a lateral pallidal lesion exacerbated akinesia; the patient was hospitalized and developed pulmonary sepsis and died of gastrointestinal haemorrhage 3 months after the procedure.

Major intracerebral haemorrhage requiring clot evacuation occurred in two cases; one of these remains hemiplegic, while the other has made a good recovery. Two minor haemorrhages, not requiring evacuation, were also encountered, both patients having good clinical results. The mean age of these four patients was 67 years. Intraoperative hypertension featured in two of these cases, and two of these haemorrhages occurred when previous lesions were being extended.

Other complications were: transient hemiparesis resolving over days to 2 weeks in three patients; dysphasia associated with dominant VIM lesions in six patients, only one of which was persistent at 3 months; and one aspiration pneumonia in a patient with multiple sclerosis and a pre-existing pseudobulbar palsy.

There was a learning curve in the size of the lesion made for tremor. In the first 20 patients treated, seven later had lesions extended, in the next 20 patients, three lesions were extended; while none of the last 20 patients were reoperated upon.

Tremor suppression was achieved in all except two multiple sclerosis patients, while highly significant $(P<0.001)$ improvements in the unified Parkinson's disease rating score and quality of life assessments were found after pallidotomy.

\section{DIsCUSSION}

In advising patients with chronic neurological disabilities about surgery, the risks and benefits of these procedues must be appreciated by all concerned with the individual's care. Like other groups, we have found this surgery to be efficacious with persistent benefits beyond 3 years. We have only encountered one death in the series, and this was not directly related to the surgery. The most serious complication in our view is that of intracerebral haemorrhage. This can be minor with good clinical results, or major and catastrophic. Our haemorrhage rate is $3.3 \%$ per lesion, but we feel that this is increased in the elderly. Intraoperative hypertension is a further risk factor, and we therefore monitor patients invasively with arterial lines during the operation, giving intravenous vasodepressors as necessary. Two of our four haemorrhages were encountered extending previously made lesions; if this is a real risk factor, it adds importance to the learning curve, as with increasing experience we have had to extend fewer lesions. In the interests of patient safety, as well as surgical efficacy, these procedures should only be undertaken by experienced practitioners performing functional neurosurgery on a frequent basis.

Overall our complication rates compare favourably with other series. ${ }^{8-11}$ We suspect that this reflects our reliance on anatomical targeting, which minimizes the amount of exploration and the number of electrode passes required to achieve a good result, and that this reduces the risk of haemorrhage.

\section{REFERENCES}

1. Cooper IS, Bravo CJ. Implications of a five year study of 700 basal ganglia operations. Neurology 1958; 8: 701-707.

2. Kelly PJ, Gillingham FJ. The long-term results of stereotactic surgery and L-dopa therapy in patients with Parkinson's Disease. J Neurosurg 1980; 53: 332-337.

3. Andrew J, Fowler CJ, Harrison MVG. Stereotaxic thalamotomy in 55 cases of dystonia.Brain 1983; 106: 981-1000.

4. Bullard DE, Nashold BS Jr. Stereotaxic thalamotomy for treatment of post traumatic movement disorders. J Neurosurg 1984; 61: 316-321.

5. Tasker RR, Yamashiro K, Lenz F, Dostrovsky JO. Thalamotomy for Parkinson's Disease:microelectrode technique.In: Lunsford LD (ed).Modern Stereotactic Neurosurgery. Boston: Mass Martinus Nijhoff; 1988: 297-314.

6. Narabayashi H, Maeda T, Yokochi F. Long term followup study of nucleus ventralis intermedius and ventrolateralis thalamotomy using a microelectrode technique in parkinsonism. Appl Neurophysiol 1987; 50: 330-337.

7. Ohye $\mathrm{C}$, Narabayashi $\mathrm{H}$. Physiological study of presumed ventralis intermedius neurons in the human thalamus. J Neurosurg 1979; 50: 290-297.

8. Obeso JA, Guridi J, DeLong M. Surgery for Parkinson's Disease. J Neurol Neurosurg Psych 1997; 62: 2-8.

9. Baron MS, Vitek JL, Bakay RAE et al.Treatment of advanced Parkinson's Disease by posterior GPi pallidotomy: 1 year results of a pilot study. Ann Neurol 1996; 40: 355-366.

10. Johannson F, Malm J, Nordh E, Hariz M.Usefulness of pallidotomy in advanced Parkinson's Disease. J Neurol Neurosurg Psych 1997; 62: 125-132.

11. Sutton JP,Couldwell W, Lew MF et al.Ventroposterior medial pallidotomy in patients with advanced Parkinson's Disease. Neurosurg 1995; 36: 1112-1117.

12. Kelly PJ. Postoperative complications of stereotactic procedures in Postoperative Complications in Intracranial Neurosurgery. $1^{\text {th }}$ ed. Stuttgart: Thieme, 1993: 166-180.

13. Scott R,Gregory R,Aziz TZ et al.Neuropsychological,neurological and functional outcome following pallidotomy for Parkinson's Disease:a consecutive series of 8 simultaneous bilateral and 12 unilateral procedures. Brain 1998; 121: 659-675.

14. Gerdes J, Hitchon P, Neerangun W, Torner J. Computed tomography versus magnetic resonance imaging in stereotactic localisation. Stereotact Funct Neurosurg 1994; 63: 124-129.

15. Kondziolka D, Dempsey $P$, Lunsford $L$ et al.A comparison between magnetic resonance imaging and computed tomography for stereotactic co-ordinate determination. Neurosurgery 1992; 30: 402 .

16. Walton L, Hampshire A, Forster, Kemeny A. A phantom study to assess the accuracy of stereotactic localisation using $\mathrm{Tl}$ weighted magnetic resonance imaging with the Leskell stereotactic system.Neurosurgery 1996; 38: 170-176.

17. Schaltenbrand G, Wahren W. Atlas for stereotaxy of the human brain. $2^{\text {nd }}$ Edition, 1977. Sturtgart: Georg Thieme Publishers,

18. Kooy HM, Van Herk M, Barnes PD, Alexander E,et al. Image fusion for stereotactic radiotherapy and radiosurgery treatment planning. Int $\mathbf{J}$ Radiation Oncology Biol Phys 1994; 28: 1229-1234. 